\title{
Back on track
}

Citation for published version (APA):

van Erp, R. M. A. (2017). Back on track: chronic low back pain rehabilitation in primary care. [Doctoral Thesis, Maastricht University]. Maastricht University. https://doi.org/10.26481/dis.20171222rve

Document status and date:

Published: 01/01/2017

DOI:

10.26481/dis.20171222rve

Document Version:

Publisher's PDF, also known as Version of record

\section{Please check the document version of this publication:}

- A submitted manuscript is the version of the article upon submission and before peer-review. There can be important differences between the submitted version and the official published version of record.

People interested in the research are advised to contact the author for the final version of the publication, or visit the DOI to the publisher's website.

- The final author version and the galley proof are versions of the publication after peer review.

- The final published version features the final layout of the paper including the volume, issue and page numbers.

Link to publication

\footnotetext{
General rights rights.

- You may freely distribute the URL identifying the publication in the public portal. please follow below link for the End User Agreement:

www.umlib.nl/taverne-license

Take down policy

If you believe that this document breaches copyright please contact us at:

repository@maastrichtuniversity.nl

providing details and we will investigate your claim.
}

Copyright and moral rights for the publications made accessible in the public portal are retained by the authors and/or other copyright owners and it is a condition of accessing publications that users recognise and abide by the legal requirements associated with these

- Users may download and print one copy of any publication from the public portal for the purpose of private study or research.

- You may not further distribute the material or use it for any profit-making activity or commercial gain

If the publication is distributed under the terms of Article $25 \mathrm{fa}$ of the Dutch Copyright Act, indicated by the "Taverne" license above, 


\section{Back on Track}

Chronic low back pain rehabilitation in primary care

Reni M. A. van Erp 
ISBN: 9789090306520

Cover design: Aart Kuipers

Typesetting: *studio Michal Slawinski

Printed in Poland

(c) 2017 R.M.A. van Erp, Veghel, the Netherlands

All rights reserved. No part of this publication may be reproduced or transmitted in any form or by any means, electronical or mechanical, including photocopy, recording or any other information storage or retrieval system, without written permission of the copyright owner.

The research presented in this thesis was conducted at CAPHRI Care and Public Health Research Institute, Department of Rehabilitation Medicine, of Maastricht University. CAPHRI participates in the Netherlands School of Primary Care Research CaRe. The Back on Track project was funded by Adelante, Centre of Expertise in Rehabilitation and Audiology Hoensbroek; the Province of Limburg (SAS-2012-01300) and CZ Foundation (AFVV12-205).

The printing of this thesis was financially supported by the Scientific College Physical Therapy (WCF) of the Royal Dutch Society for Physical Therapy (KNGF). 


\title{
Back on Track \\ Chronic low back pain rehabilitation in primary care
}

\author{
Proefschrift \\ ter verkrijging van de graad van doctor aan de Universiteit Maastricht, \\ op gezag van de Rector Magnificus, Prof. dr. Rianne M. Letschert \\ volgens het besluit van het College van Decanen, \\ in het openbaar te verdedigen \\ op vrijdag 22 december 2017 om 10.00 uur \\ door
}

Reni Maria Arnoldina van Erp 


\section{PROMOTORES}

Prof. dr. R.J.E.M. Smeets

Prof. dr. J.A. Verbunt

COPROMOTOR

Dr. I.P.J. Huijnen

\section{BEOORDELINGSCOMMISSIE}

Prof. dr. D. Ruwaard (voorzitter)

Prof. dr. R.A. de Bie

Dr. M.E.J.B. Goossens

Prof. dr. R.W.J.G. Ostelo (Vrije Universiteit Amsterdam)

Prof. dr. D.A.W.M. van der Windt (Keele University, United Kingdom) 


\section{Table of contents}

$\begin{array}{ll}\text { Chapter } 1 & 7\end{array}$

General introduction

Chapter 2

Effectiveness of primary care interventions using a biopsychosocial approach in chronic low back pain: a systematic review

\section{Chapter 3}

Development and content of the biopsychosocial primary care intervention 'Back on Track' for a subgroup of people with chronic low back pain

\section{Chapter 4}

A biopsychosocial primary care intervention (Back on Track) versus primary care as usual in a subgroup of patients with chronic low back pain; protocol for a randomised controlled trial

\section{Chapter 5}

Biopsychosocial primary care and physiotherapy as usual show no differences in effects in patients with chronic low back pain: results of a randomised controlled trial

\section{Chapter 6}

Feasibility of the biopsychosocial primary care intervention 'Back on Track' for patients with chronic low back pain: a process and effect-evaluation

\section{Chapter 7}

Spinal surgeons' opinions on pre- and postoperative rehabilitation in patients undergoing lumbar spinal fusion surgery: a surveybased study in the Netherlands and Sweden

\section{Chapter 8}

General discussion

\section{Chapter 9}

Valorisation addendum

\section{Summary}



Chapter 1

General introduction 



\section{GENERAL INTRODUCTION}

Back and neck pain are common health problems, having even the highest incidence rate of all health problems in the Netherlands (incidence rate of 852.630 in 2011). ${ }^{1}$ Low back pain (LBP) is generally defined as "pain and discomfort, localised below the costal margin and above the inferior gluteal folds, with or without referred leg pain".2 LBP has a mean point prevalence of $11.9 \pm 2.0 \%$ and a mean one-year prevalence of $23.2 \pm 2.9 .^{3}$ Furthermore, a lifetime prevalence of up to $84 \%$ has been reported, indicating that most people will experience LBP at some point in their lives. ${ }^{2,4}$ In only $10 \%$ of the patients who experience LBP, a specific pathology for the LBP can be found. ${ }^{5}$ Examples of specific pathology are nerve root involvement or serious spinal pathology (e.g. tumour, infection or inflammatory disease). In the other $90 \%$ of the patients, LBP is due to a "simple" back problem. In this case, no serious spinal pathology is present. ${ }^{2}$ Such LBP is also called "non-specific LBP". Although there is still limited understanding about what actually causes non-specific LBP, patients and physiotherapists consider biomechanical factors (e.g. lifting, bending, and rotating) to be the most important trigger. ${ }^{6}$

Non-specific LBP with a duration of 6 weeks or less, is called acute LBP. The prognosis for acute LBP is initially good as the pain and disability mostly reduce remarkably in the first six weeks.? However, once having experienced LBP, the chance of a new episode is approximately twice as high as compared to people without a history of LBP. ${ }^{8}$ Recurrent LBP is therefore common. If a LBP episode has a duration of 6 up till 12 weeks, the LBP is called subacute. In case complaints persist for more than 12 weeks, the LBP is called chronic (CLBP)., ${ }^{910}$ This thesis specifically focuses on this subgroup of patients with a chronic stage of LBP; patients who experience non-specific CLBP.

\section{CHRONIC LOW BACK PAIN}

In about one-quarter of the patients who experience acute LBP, the complaints become chronic.." A combination of biological, psychological and/or social factors is suggested to act as an underlying mechanism for the ongoing pain and disability. ${ }^{9}$ Specific risk factors for the development of CLBP are for example a high baseline level of pain and disability, maladaptive coping behaviour (e.g. fear-avoidance beliefs), somatisation, low general health status, and psychiatric comorbidity. ${ }^{11}$ As such bio-psycho-social factors can play a significant role in the development and persistence of CLBP, national and international therapy guidelines for LBP recommend assessment of psychosocial factors (also described as "yellow flags") within the diagnostic triage of patients with LBP. After having defined the presence and influence of biopsychosocial factors, therapy should be adjusted to the patient's biopsychosocial profile.

A biopsychosocial model, commonly used in Dutch clinical practice, which can be helpful to discuss and unravel a patient's LBP problem, is the pain-consequence model (Figure 1). The left side defines what factors have caused the LBP and what biomedical strategies have been used to reduce the pain and disability (biomedical approach). The right side of the model defines how a patient behaves or copes with the pain, and to what extent this behaviour has influenced physical, social and cognitive domains over time (i.e. short and long term consequences; biopsychosocial approach). 
Figure 1 The pain-consequence model

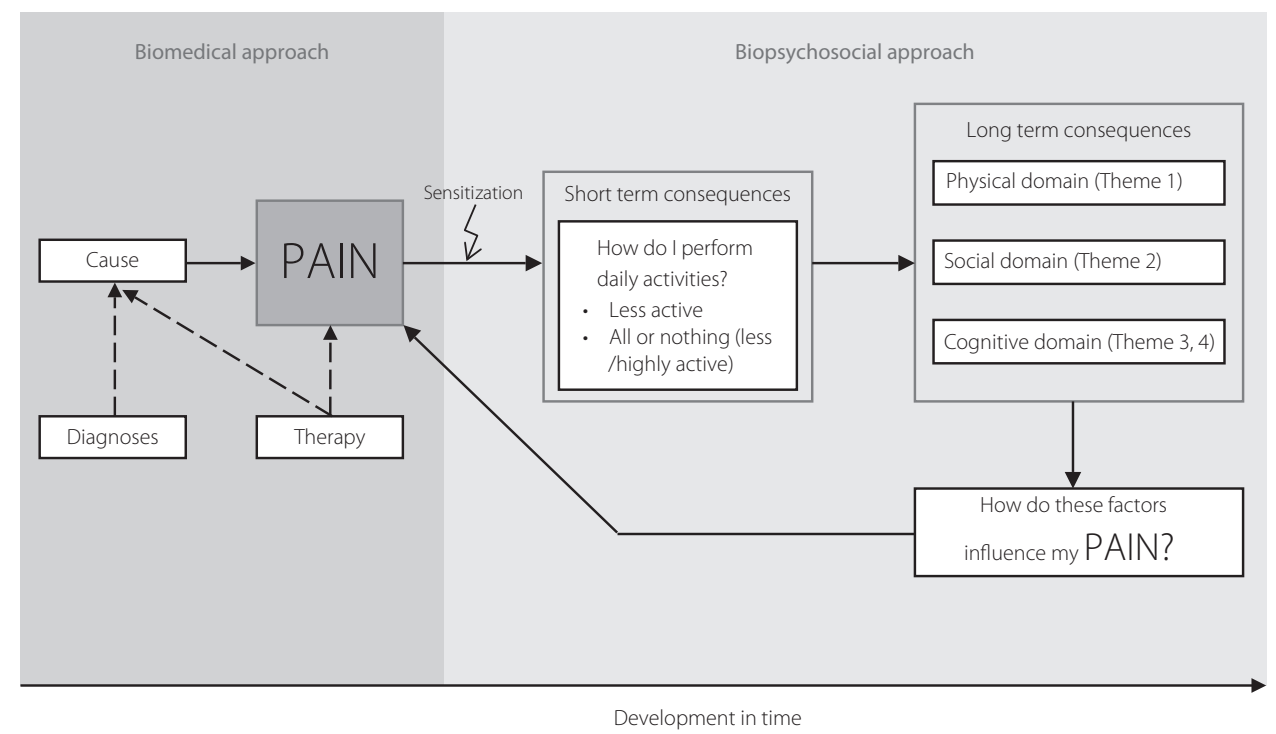

From a biomedical view, acute pain is caused by noxious chemical, mechanical or thermal stimuli. Chemicals will be released due to the noxious stimuli which activate nociceptors. Activated nociceptors will transmit the noxious information via the dorsal horn of the dorsal root to the spinal cord and towards the brain. In line with the model of Loeser, brain areas process the noxious information, which results in a cortical perception of pain. ${ }^{12}$ In a subgroup of patients, the sensory processes of the nervous system may be altered. One potential underlying mechanism for this is central sensitization. ${ }^{13}$ Central sensitization is defined by The International Association for the Study of Pain (IASP) as "an increased responsiveness of nociceptive neurons in the central nervous system to their normal or subthreshold afferent input".14 Patients with central sensitization thus seem hypersensitive to peripheral (chemical, mechanical, and thermal) stimuli. Although nociception and the cortical perception of pain might be more of "biomedical nature", the conscious experience or awareness of pain is eventually more of "biopsychosocial nature". Personal factors such as beliefs, mood, personality, and attitude, as well as opinions or responses from family, friends, colleagues or health care professionals can influence how a person experiences pain but also how pain is processed as these factors all can influence the efferent pain inhibition system. One might have catastrophizing thoughts and feel depressed about the (persisting) pain, while another might still be positive minded and feels less depressed. The experience of pain finally results in a certain type of pain behaviour. Several types of behaviour have been suggested in pa- 
tients with $\mathrm{CLBP}^{15,16}$ of which the pain-consequence model presents two (as a stimulus to define/ discuss the specific behaviour of the patient). One type of behaviour described in the pain-consequence model is "less active behaviour". This type of behaviour can be related to fear-avoidance behaviour, explained by the Fear Avoidance Model. ${ }^{17}$ This model proposes that after the experience of pain (e.g. during lifting), a person can develop catastrophizing thoughts about this activity (e.g. lifting will cause my back to fracture). Catastrophizing thoughts may lead to fear or anxiety for this specific activity (pain-related fear). Eventually this pain-related fear can lead to hypervigilance (increased sensitivity or attention to threat-related stimuli) and to the avoidance of this activity (e.g. avoidance of lifting).

In contrast to avoiding activities, the second type of behaviour, "all or nothing behaviour", can be related to endurance behaviour. Endurance behaviour is characterised by ignoring or suppressing the pain. A person with this behavioural style persists in being active and continues until the task, activity or goal is completed despite the pain. Although this type of behaviour is less scientifically investigated and underpinned than the fear-avoidance behaviour, both types of behaviour have consequences at short and long term. As avoiding activities will prevent a person from the experience of pain, this behaviour seems beneficial at short term. However, at long term this behaviour can lead to e.g. higher levels of disability (physical), depressed mood (cognitive) or social isolation (social). Also in the case of the "all or nothing" behaviour, a person might have feelings of control at short term. However, at long term this person might experience more pain (due to overusing/overloading the body), lose feelings of control and take rest until the pain disappears. In a person with an "all or nothing", pain-dependent behaviour, a saw-tooth activity pattern is seen in which highly active behaviour is alternated with less active behaviour. Eventually, as a consequence of this behaviour, this person also becomes less active and more functionally disabled at long term.

\section{THERAPY APPROACHES}

To what extent previous mentioned psychosocial factors lead to certain behaviour, to an increased level of disability and eventually to the chronification of LBP, can vary considerable between patients. The need for therapy and the content, intensity and setting of this therapy (i.e. primary, secondary or tertiary) might therefore differ. Hence, the Dutch special interest group on pain rehabilitation (Werkgroep Pijnrevalidatie Nederland; WPN) designed a classification system for physicians in rehabilitation medicine to subgroup individuals with chronic pain into four classes (Table 1). ${ }^{18,19}$ This classification is based on the contributing role of psychosocial factors and the level of functional disability. Lower classifications (WPN1 - WPN2) indicate no or less complex psychosocial problems and no or lower levels of functional disability, while higher classifications (WPN3 - WPN4) indicate the presence of more complex psychosocial problems and higher levels of functional disability. 
Table 1 Classification system and health care policy in the Netherlands for patients with CLBP based on the psychosocial profile and the level of functional disability

\begin{tabular}{clll}
\hline $\begin{array}{l}\text { WPN } \\
\text { level }\end{array}$ & Psychosocial factors & $\begin{array}{l}\text { Disability } \\
\text { level }\end{array}$ & Health care policy/therapy setting \\
\hline 1 & Not present (or of very low complexity) & - & Primary care (general practitioner) \\
2 & Low complexity & Low & Primary care (physiotherapist) \\
3 & Moderate complexity & Moderate & Secondary care (multidisciplinary) \\
4 & High complexity & High & Secondary/tertiary care (multidisciplinary) \\
\hline
\end{tabular}

Based on this classification system, physicians refer patients to a specific therapy, which is considered most suitable. A patient classified as WPN1 (i.e. without psychosocial factors), will not be referred to treatment and receives advice only. This patient is advised to stay active and reassured that nothing is seriously wrong with the lower back. A patient classified as WPN2 (i.e. with psychosocial factors that influence daily life functioning minimally) is referred to a physiotherapy treatment provided in a primary care physiotherapy practice. The Dutch primary care physiotherapy guideline recommends advice to stay active and supervised exercises. ${ }^{10}$ It has become evident that Dutch physiotherapists seem to deliver exercise therapy and manual interventions mainly in daily practice (e.g. manipulations and massage). ${ }^{20} \mathrm{~A}$ patient classified as WPN3 or WPN4 (i.e. with moderate to complex psychosocial factors) is generally referred to a pain rehabilitation programme in a secondary or tertiary care setting such as a hospital, rehabilitation centre and independent treatment centre (Zelfstandige Behandel Centrum, ZBC). Such programmes are generally offered multidisciplinary (or nowadays often referred as interdisciplinary) and focus specifically on targeting the impact of psychosocial factors and on improving the level of activities despite pain. A team of health care professionals who are specialised and experienced in using cognitive-behavioural approaches deliver these treatments.

In line with the European guideline for the management of non-specific CLBP, invasive treatments such as acupuncture, nerve blocks or injection therapy are not indicated. ${ }^{2}$ Also surgery such as spinal fusion surgery, is initially not recommended. Despite this, the number of fusion surgeries in patients with CLBP has increased during the last two decades. ${ }^{21,22}$ It should however be stressed that, at least in patients with CLBP and degenerative disc disease (DDD), cognitive therapy in combination with exercises still seems to be preferred over e.g. spinal fusion surgery as it results in similar short and long term improvements in functional disability and less complications. $^{23-25}$

\section{BIOPSYCHOSOCIAL THERAPY APPROACHES}

The cognitive-behavioural approaches that are frequently used within (conservative) multidisciplinary pain rehabilitation programmes for patients with CLBP are for example Graded Activity (GA), Exposure in vivo (EXP), and Acceptance and Commitment Therapy (ACT). GA is based on operant conditioning and aims to increase the level of activities by reinforcing healthy behaviour (positive reinforcement). ${ }^{26,27}$ It starts by defining the baseline activity level of functional 
activities and by setting a patient-specific plan. The patient-specific plan eventually stimulates the patient to a time-contingent, rather than pain-contingent, increase of the level of activities. EXP is originally based on classical conditioning and is related to the fear-avoidance model. ${ }^{17}$ EXP aims to identify patient's (catastrophizing) cognitions about certain movements or activities and subsequently exposes a patient to these fearful activities. EXP stimulates a patient to readjust the associated cognitions and by doing so, to reduce avoidance behaviour (i.e. becoming more active). ACT uses a different approach and focuses on acceptance of the CLBP and personal values in life (commitment) and aims to increase psychological flexibility. ${ }^{28}$

Multidisciplinary pain rehabilitation with such a biopsychosocial focus seems to be effective and more promising than advice from general practitioners or physiotherapy in reducing disability and pain in patients with CLBP. ${ }^{29}$ In the Netherlands, the number of multidisciplinary pain rehabilitation programmes for patients with CLBP within hospitals, rehabilitation centres and independent treatment centres (ZBC's) have increased during the past decade. While in 2002 only $7 \%$ of the outpatient rehabilitation was directed to the treatment of chronic pain patients (i.e. number of patients), this number increased to $25 \%$ in 2011. A drawback of a multidisciplinary pain rehabilitation programme is that it can be expensive due to the extensive treatment programmes in which multiple disciplines are involved. For example, the cost per chronic pain rehabilitation (per patient) is on average $€ 2,905$ in Dutch hospitals and rehabilitation centres, and even $€ 8,760$ in independent treatment centres (ZBC's). ${ }^{30}$ At this moment, multidisciplinary pain rehabilitation programmes therefore put a major financial burden on the health care system in the Netherlands. In combination with the aging, growing and demanding population, it is expected that the number of patients with $\mathrm{CLBP}^{3}$ and therefore the number of patients treated in multidisciplinary settings will increase. This will subsequently lead to an increased financial burden of multidisciplinary programmes on the health care system in future. ${ }^{31}$

The National Health Care Institute in the Netherlands (Zorginstituut Nederland) and the Dutch Ministry of Health, Welfare and Sport (VWS) stressed the need for strategies to reduce this financial burden. ${ }^{31}$ One potential strategy is to stimulate biopsychosocial interventions delivered by physiotherapists in primary care physiotherapy settings. An advantage of a primary care physiotherapy treatment is that it is generally easy accessible. Furthermore, the waiting time is mostly limited (stimulating early rehabilitation), and the direct therapy costs per session are lower as compared to multidisciplinary secondary care sessions.

At the start of this thesis, less was clear about the evidence for a biopsychosocial intervention in a primary care physiotherapy practice for patients with CLBP. It remained to be investigated which studies had previously investigated the effects of a biopsychosocial primary care intervention. To get insight in previously performed studies, performing a systematic review was considered essential. We noticed that only few biopsychosocial primary care physiotherapy interventions had been developed and were evaluated in a Randomised Controlled Trial (RCT). In addition, it became evident that biopsychosocial interventions varied considerably in design (e.g. the cognitive-behavioural approach used) and the education and skill of physiotherapists who delivered the intervention, what may have influenced the quality of delivery. There was need for the development of a new biopsychosocial primary care intervention based on the available scientific evidence and expert opinions, and in addition, to evaluate its effectiveness as compared 
to a usual physiotherapy programme in primary care in patients usually referred to primary care physiotherapy interventions (i.e. patients classified as WPN2). In addition, with the potential financial advantages of a primary care intervention in mind, it was questioned whether a biopsychosocial primary care intervention would be feasible in patients who normally would be referred to a multidisciplinary secondary care intervention (i.e. patients classified as WPN3-). As this would be a new approach for a patient classified as WPN3-, it was unclear which practical factors would influence the delivery and receipt of the intervention. Therefore, a process evaluation alongside an effect evaluation was considered valuable to study the feasibility and potential beneficial effects.

\section{BIOPSYCHOSOCIAL THERAPY APPROACHES AND LUMBAR SPINAL FUSION SURGERY}

Even though conservative treatments are recommended for patients with CLBP, it was noticed that lumbar spinal fusion surgery is increasingly provided in patients with CLBP nowadays, more specifically in patients with signs of DDD or spondylolisthesis. ${ }^{32,33}$ The general aim of lumbar spinal fusion surgery is to fuse and thereby stabilise the painful vertebrae and intervertebral discs, and by doing so reduce the pain. Although this is a rather biomedical approach and initially not recommended, a biopsychosocial approach might add value in the pre- and post-operative phase. Recent evidence suggests for example beneficial effects of pre-operative rehabilitation ("prehabilitation") on postoperative recovery, ${ }^{34}$ and shows that focusing on physical as well as cognitive-behavioural aspects (e.g. catastrophizing, fear of movement) in the pre-operative phase is beneficial for faster mobilisation and pain-coping postoperative. ${ }^{35}$ Also in the postoperative phase, treatments with a cognitive-behavioural approach seem to reduce disability, fear-avoidance beliefs and self-efficacy more effectively than exercise programmes only. ${ }^{36}$ Specific guidelines for preand postoperative rehabilitation are, however, not available. Moreover, it is unclear whether such pre- or postoperative programmes are actually advised by spinal surgeons in clinical practice, and to what extent spinal surgeons provide advice about e.g. mobilisation, daily activities, work and sports (allowed/not allowed, when, to what intensity, etcetera). Research is therefore needed to investigate what opinions spinal surgeons have about pre- and postoperative rehabilitation in patients undergoing lumbar spinal fusion surgery and what they recommend in daily practice. Such information is expected to be useful for new studies to investigate which pre- and postoperative approach is most effective and for developing practical guidelines in future.

\section{AIMS AND OUTLINE OF THIS DISSERTATION}

In order to get insight in the current available evidence for biopsychosocial primary care physiotherapy interventions for patients with non-specific CLBP, the first aim of this thesis was to systematically review and synthesise the available literature (Chapter 2). Apart from investigating to what extent biopsychosocial interventions delivered by primary care physiotherapists have an effect on disability, pain and work status as compared to other primary care interventions, we also aimed to provide an overview of the content of each biopsychosocial therapy programme, the training programme of physiotherapists and other process-related factors that might influence 
practical implementation. We noticed that only few studies investigated the effectiveness for a biopsychosocial intervention in primary care with methodological and practical limitations, and noticed that new high-quality studies were needed. Therefore, our second aim was to develop a new biopsychosocial primary care intervention 'Back on Track'. The development and content is presented in Chapter 3. We subsequently set up a protocol for a Randomised Controlled Trial (RCT) in which we aimed to compare the effectiveness of this newly developed Back on Track intervention with physiotherapy as usual for patients classified as WPN2 (Chapter 4). Results of this RCT will be presented in Chapter 5. In addition to the RCT, we aimed to perform a feasibility study to investigate to what extent the newly developed Back on Track intervention is feasible and effective for patients classified as WPN3- (Chapter 6). Since referring physician in rehabilitation medicine are more closely involved in the treatment process of patients classified as WPN3-, the Back on Track intervention for this subgroup of patients was designed as an integrated care intervention. An additional and final aim of the research project was to gather opinions of Dutch and Swedish spinal surgeons regarding pre- and postoperative rehabilitation in patients undergoing lumbar spinal fusion surgery of which the results are presented in Chapter 7.

Overall, the objectives and outline of this thesis are:

- To systematically review the evidence on the effectiveness of biopsychosocial primary care interventions in patients with CLBP (Chapter 2).

- To give a detailed overview of the development and content of the biopsychosocial primary care intervention Back on Track (Chapter 3).

- To describe the rationale and design of the RCT comparing the Back on Track intervention with physiotherapy as usual for patients with CLBP (Chapter 4).

- To determine the short term effectiveness (3 months) of the Back on Track intervention as compared to primary care as usual in patients with CLBP experiencing low complex psychosocial complaints (Chapter 5).

- To describe the feasibility and effectiveness of the Back on Track intervention in patients with CLBP experiencing moderate complex psychosocial complaints (Chapter 6).

- To provide insight in the opinions of Dutch and Swedish spinal surgeons about pre- and postoperative lumbar spinal fusion rehabilitation (Chapter 7).

- To finish with a general discussion about the main findings, conclusions and practical recommendations (Chapter 8), a summary (English and Dutch), and possibilities for valorisation/implementation. 


\section{REFERENCES}

1. RIVM. Ranglijst ziekten op basis van incidentie. 2011; https://www.volksgezondheidenzorg.info/ranglijst/ranglijst-ziekten-op-basis-van-incidentie. Accessed 24072017.

2. Airaksinen O, Brox Jl, Cedraschi C, et al. Chapter 4. European guidelines for the management of chronic nonspecific low back pain. European spine journal : official publication of the European Spine Society, the European Spinal Deformity Society, and the European Section of the Cervical Spine Research Society. 2006;15 Suppl 2:S192-300.

3. Hoy D, Bain C, Williams G, et al. A systematic review of the global prevalence of low back pain. Arthritis and rheumatism. 2012;64(6):2028-2037.

4. Balague F, Mannion AF, Pellise F, Cedraschi C. Non-specific low back pain. Lancet. 2012;379(9814):482491.

5. Maher C, Underwood M, Buchbinder R. Non-specific low back pain. Lancet. 2017;389(10070):736-747.

6. Stevens ML, Steffens D, Ferreira ML, et al. Patients' and Physiotherapists' Views on Triggers for Low Back Pain. Spine. 2016;41(4):E218-224.

7. da CMCL, Maher CG, Hancock MJ, McAuley JH, Herbert RD, Costa LO. The prognosis of acute and persistent low-back pain: a meta-analysis. CMAJ : Canadian Medical Association journal = journal de I'Association medicale canadienne. 2012;184(11):E613-624.

8. Hestbaek L, Leboeuf-Yde C, Manniche C. Low back pain: what is the long-term course? A review of studies of general patient populations. European spine journal : official publication of the European Spine Society, the European Spinal Deformity Society, and the European Section of the Cervical Spine Research Society. 2003;12(2):149-165.

9. Waddell G. The back pain revolution. 2nd ed. ed. Oxford: Churchill Livingstone; 2004.

10. Staal JB, M. HEJ, Heijmans M, et al. KNGF-richtlijn Lage-rugpijn. Amersfoort: Drukkerij De Gans;2013.

11. Chou R, Shekelle P.Will this patient develop persistent disabling low back pain? JAMA. 2010;303(13):12951302.

12. Loeser JD. Perspectives on pain. In: Clinical Pharmacology \& Therapeutics. London: Palgrave Macmillan UK; 1980:316-326.

13. Nijs J, Apeldoorn A, Hallegraeff $H$, et al. Low back pain: guidelines for the clinical classification of predominant neuropathic, nociceptive, or central sensitization pain. Pain Physician. 2015;18(3):E333-346.

14. Merskey H, Bogduk N. Classification of Chronic Pain. Part III: Pain Terms, A Current List with Definitions and Notes on Usage. 1994:209-214.

15. Hasenbring MI, Verbunt JA. Fear-avoidance and endurance-related responses to pain: new models of behavior and their consequences for clinical practice. The Clinical journal of pain. 2010;26(9):747-753.

16. Huijnen IP, Verbunt JA, Peters ML, et al. Differences in activity-related behaviour among patients with chronic low back pain. European journal of pain. 2011;15(7):748-755.

17. Vlaeyen JW, Kole-Snijders AM, Boeren RG, van Eek H. Fear of movement/(re)injury in chronic low back pain and its relation to behavioral performance. Pain. 1995;62(3):363-372.

18. Boonstra AM, Bühring M, Brouwers M, Bosma F, Schiphorst Preuper R. Patiënten met chronische pijnklachten op het grensvlak van revalidatiegeneeskunde en psychiatrie. Ned Tijdschr Pijn bestr. 2008;27(34):5-9. 
19. Boonstra AM, Schiphorst Preuper HR, Brouwer M, Weerdt de M, Knol DL, Köke A. Classificatie van patiënten met chronische pijnklachten; onderzoek naar de betrouwbaarheid van de WPN niveaus. Nederlandstalig Tijdschrift Pijnbestrijding. 2011;30(46):5-10.

20. Swinkels IC, van den Ende CH, van den Bosch W, Dekker J, Wimmers RH. Physiotherapy management of low back pain: does practice match the Dutch guidelines? The Australian journal of physiotherapy. 2005;51(1):35-41.

21. Rajaee SS, Bae HW, Kanim LE, Delamarter RB. Spinal fusion in the United States: analysis of trends from 1998 to 2008. Spine. 2012;37(1):67-76.

22. Machado GC, Maher CG, Ferreira PH, et al. Trends, Complications, and Costs for Hospital Admission and Surgery for Lumbar Spinal Stenosis. Spine. 2017.

23. Brox II, Sorensen R, Friis A, et al. Randomized clinical trial of lumbar instrumented fusion and cognitive intervention and exercises in patients with chronic low back pain and disc degeneration. Spine. 2003;28(17):1913-1921.

24. Brox Jl, Reikeras $\mathrm{O}$, Nygaard O, et al. Lumbar instrumented fusion compared with cognitive intervention and exercises in patients with chronic back pain after previous surgery for disc herniation: a prospective randomized controlled study. Pain. 2006;122(1-2):145-155.

25. Brox JI, Nygaard OP, Holm I, Keller A, Ingebrigtsen T, Reikeras O. Four-year follow-up of surgical versus non-surgical therapy for chronic low back pain. Annals of the rheumatic diseases. 2010;69(9):1643-1648.

26. Lindstrom I, Ohlund C, Eek C, et al. The effect of graded activity on patients with subacute low back pain: a randomized prospective clinical study with an operant-conditioning behavioral approach. Physical therapy. 1992;72(4):279-290; discussion 291-273.

27. Fordyce WE. Behavioral methods for chronic pain and illness. St. Louis: CV Mosby; 1976.

28. Hayes SC. Acceptance and Commitment Therapy, Relational Frame Theory, and the Third Wave of Behavioral and Cognitive Therapies-Republished Article. Behav Ther. 2016;47(6):869-885.

29. Kamper SJ, Apeldoorn AT, Chiarotto A, et al. Multidisciplinary biopsychosocial rehabilitation for chronic low back pain. The Cochrane database of systematic reviews. 2014;9:CD000963.

30. Van Eijndhoven M, Gaasbeek Janzen M, Latta J, De Wit J. Rapport Medisch-specialistische revalidatie: zorg zoals revalidatieartsen plegen te bieden. Zorginstituut Nederland;2015.

31. Onderhandelaarsresultaat medisch specialistische zorg 2014 t/m 2017. In. Den Haag: VWS; 2013.

32. Jacobs WC, Rubinstein SM, Koes B, van Tulder MW, Peul WC. Evidence for surgery in degenerative lumbar spine disorders. Best practice \& research Clinical rheumatology. 2013;27(5):673-684.

33. Stromqvist B, Fritzell P, Hagg O, Jonsson B, Sanden B, Swedish Society of Spinal S. Swespine: the Swedish spine register : the 2012 report. European spine journal : official publication of the European Spine Society, the European Spinal Deformity Society, and the European Section of the Cervical Spine Research Society. 2013;22(4):953-974.

34. Nielsen PR, Jorgensen LD, Dahl B, Pedersen T, Tonnesen $H$. Prehabilitation and early rehabilitation after spinal surgery: randomized clinical trial. Clinical rehabilitation. 2010;24(2):137-148.

35. Rolving N, Nielsen CV, Christensen FB, Holm R, Bunger CE, Oestergaard LG. Preoperative cognitive-behavioural intervention improves in-hospital mobilisation and analgesic use for lumbar spinal fusion patients. BMC musculoskeletal disorders. 2016;17:217.

36. Abbott AD, Tyni-Lenne R, Hedlund R. Early rehabilitation targeting cognition, behavior, and motor function after lumbar fusion: a randomized controlled trial. Spine. 2010;35(8):848-857. 



\title{
Chapter 2
}

\author{
Effectiveness of primary care \\ interventions using a biopsychosocial \\ approach in chronic low back \\ pain: a systematic review
}

\author{
Reni van Erp \\ Ivan Huijnen \\ Marluuke Jakobs \\ Jos Kleijnen \\ Rob Smeets
}





\section{ABSTRACT}

Background and Objective: Recent systematic reviews show promising effects for multidisciplinary biopsychosocial (biopsychosocial) interventions in patients with chronic low back pain (CLBP). Nowadays, biopsychosocial interventions have also been developed for primary care physiotherapy settings. Our aim was to systematically review the evidence on the effectiveness of primary care biopsychosocial interventions in improving functional disability, pain and work status for patients with CLBP. Secondly, we aimed to provide an overview of biopsychosocial intervention designs, physiotherapist-training programmes and process-related factors (practical implementation).

Databases and Data Treatment: We searched in scientific databases and reference lists. Randomised Controlled Trials (RCTs) evaluating primary care physiotherapist-led biopsychosocial interventions in adults ( $\geq$ 18 years) with non-specific CLBP ( $\geq 12$ weeks) were included.

Results: Searches resulted in 943 references; 7 RCTs were finally included (1,426 participants). Results show moderate quality evidence (3 trials; 991 participants) that a biopsychosocial intervention is more effective than education/advice for reducing disability and pain in the short, medium, and long term. Low quality evidence (4 trials; 435 participants) was found for no difference with physical activity treatments.

Conclusions: biopsychosocial interventions seem more effective than education/advice, and as effective as physical activity interventions in patients with CLBP. biopsychosocial interventions with a clear focus on psychosocial factors (understanding pain, unhelpful thoughts, coping styles, and goal-setting) seem most promising. Sufficient delivery of biopsychosocial elements is expected when physiotherapist receive training programmes with extensive support prior and during delivery (manual, supervision, and informative resources). Future studies of sufficient quality are expected to add significant information.

\section{KEYWORDS}

Low back pain, chronic pain, biopsychosocial intervention, primary health care, physiotherapy, systematic review 


\section{INTRODUCTION}

Chronic low back pain (CLBP) is defined as a health condition in which low back pain persists for $\geq$ 12 weeks. ' CLBP can be very disabling at an individual's functional, psychological, and social level. Moreover, it has a large impact on society due to the related high health care consumption and work absenteeism. ${ }^{2}$ The recognition that many of these factors can influence a patient's low back pain problem led to the development of the biopsychosocial model. ${ }^{3}$ Subsequently, multiple interventions with a biopsychosocial approach have been developed to target influencing factors, aiming to improve the level of a patient's daily life functioning. ${ }^{4}$

Within a biopsychosocial treatment, cognitive-behavioural approaches are often used, such as graded activity, exposure in vivo, ${ }^{6}$ or acceptance and commitment therapy. ${ }^{7.8}$ Biopsychosocial interventions are frequently provided by multidisciplinary teams in rehabilitation centres or specialised pain clinics for patients with moderate to high levels of disability. Multidisciplinary biopsychosocial interventions are more effective in reducing pain and disability as compared to usual care (e.g. provided by a general practitioner (GP)) or physical treatment (e.g. passive or active therapy provided by a physiotherapist). ${ }^{4}$ Due to the promising effects of such biopsychosocial interventions, similar interventions have also been developed for primary care (physiotherapy) settings.

Treatments in primary care physiotherapy settings are easy accessible, less expensive than multidisciplinary care (therapy costs) and often have shorter waiting lists. Few systematic reviews investigated the evidence from existing biopsychosocial primary care interventions. They focused on biopsychosocial interventions in patients with low back pain in general (i.e. acute, subacute and chronic), ${ }^{9}$ or (sub)acute low back pain specifically. ${ }^{10,11}$ Until now, no systematic review has been performed to investigate the evidence of biopsychosocial interventions provided by physiotherapists working in primary care for CLBP specifically. In addition, less attention has been paid to factors other than methodological ones that might influence outcomes as well. It seems for example that physiotherapists working in primary care settings prefer a straightforward biomedical approach or feel unprepared to use a biopsychosocial approach (i.e. identifying and modifying influencing psychosocial factors). ${ }^{12}$ Besides skills or preparation/training of physiotherapists, adherence to the protocol or other factors can influence implementation of the intervention. As this can eventually influence improvement of a patient and thus outcome, it is important to have insight into practical factors for a better understanding of the process and the interpretation of the results of a study. Therefore, the aim of the current systematic review is:

1. To provide an overview of the evidence of randomised controlled trials (RCTs) for the effectiveness of primary care biopsychosocial interventions compared with waiting list controls or other primary care treatments in improving functional disability, pain and work status of patients with CLBP; and

2. To provide an overview of the design of each biopsychosocial intervention, the profiles of physiotherapists, the biopsychosocial education/training programmes for therapists, as well as of other factors such as protocol adherence and therapy compliance. 


\section{METHODS}

The systematic review was performed following the Cochrane Collaboration guidelines ${ }^{13,14}$ and reported using The PRISMA statement. ${ }^{15}$ The protocol was registered in the International Prospective Register of Systematic Reviews (PROSPERO; available from http://www.crd.york.ac.uk/ PROSPERO/; registration number: CRD42015029878; 08.12.2015).

\section{ELIGIBILITY CRITERIA}

\section{Types of studies}

English, Dutch and German language, full-text and peer-reviewed randomised controlled trials studying the effectiveness of biopsychosocial primary care treatment in patients with CLBP were included. No publication date or publication status restrictions were imposed.

\section{Types of participants}

Studies with adult participants ( $\geq 18$ years) experiencing non-specific CLBP were included. Non-specific CLBP was defined as pain between the $12^{\text {th }}$ rib and gluteal region, whether or not with radiation towards one or both legs, present for at least three months. ${ }^{3}$ Studies evaluating patients with acute and subacute low back pain were excluded unless these subjects comprised $10 \%$ or less of the total study population ( $\geq 90 \%$ should be CLBP), or results of patients with CLBP were presented separately.

\section{Types of interventions}

We defined a biopsychosocial intervention as a multicomponent intervention including at least (1) a biological component e.g. to improve (knowledge of) physical components, pain physiology, pain sensitization, differences between acute and chronic, and (2) a psychological or social component e.g. to improve (knowledge about the influence of) cognitions, attitude, (pain-) behaviour, coping styles, (self-) management strategies, and/or coping styles of family/friends/ colleagues. This definition is in line with definitions used in the literature and a recently-conducted systematic review. ${ }^{3,4}$ Interventions had to be provided individually or in a group and delivered by a physiotherapist working in primary care. Studies evaluating multidisciplinary interventions delivered by different health care professionals were excluded. Primary care treatments without a biopsychosocial approach (e.g. usual care, physical treatment) or no treatment/waiting list were considered as control interventions. 


\section{Types of outcome measures}

In accordance with recent conducted reviews on multidisciplinary rehabilitation treatments $s^{4,16}$ and as recommended by the Cochrane Back Review Group, ${ }^{13}$ primary outcomes included patient-centred measures such as functional disability, pain, and work status. Secondary outcomes included generic functional status or wellbeing, overall improvement or satisfaction, emotional functioning and cognitions (depression, anxiety, catastrophizing, fear-avoidance), and adverse events (AEs). Outcomes were categorised as short term (up to 3 months), medium term ( $>3$ to 12 months), and long term (> 12 months).

\section{SEARCH METHODS FOR IDENTIFICATION OF STUDIES}

A search strategy was developed by review authors (RVE, IH, JK, RS). The search strategy included indexed keywords (e.g. Medical Subject Headings, MeSH) and text terms for title and/or abstract of database records. An information specialist (SD) finalised the search strategy, adapted keywords according to the configuration of each database, and performed the computer-aided search (Appendix I, 30.11.15 - 01.12.2015). The following databases were used:

- MEDLINE (Ovid)

- MEDLINE In-Process Citations \& Daily Update (Ovid)

- PubMed (NLM) (Internet) http://www.ncbi.nlm.nih.gov/pubmed

- Embase (Ovid)

- PsycINFO (Ovid)

- CINAHL (EBSCO)

- Cochrane Database of Systematic Reviews (CDSR) (Cochrane Library: Wiley)

- The Cochrane Central Register of Controlled Trials (CENTRAL) (Cochrane Library: Wiley)

- Database of Abstracts of Reviews of Effects (DARE) Cochrane Library: Wiley)

- Health Technology Assessment (HTA) database (Cochrane Library: Wiley)

- PEDro (Internet) http://www.pedro.org.au/

Reference lists of review articles and included articles were checked on relevant studies. Identified references were downloaded and collected using the EndNote bibliographic software.

\section{STUDY SELECTION}

Two review authors (RVE and MJ) independently screened and selected search results by title and abstract based on previously formulated eligibility criteria. A third review author $(\mathrm{IH})$ was consulted to resolve disagreement. Subsequently, selected searches were independently screened as full text by two review authors (RVE and MJ) and if necessary by a third review author $(I H)$ to gain consensus. 


\section{RISK OF BIAS ASSESSMENT}

Two review authors (RVE and MJ) independently performed the risk of bias assessment and a third review author $(\mathrm{IH})$ was involved in case of disagreement. The Cochrane Back Review Group (CBRG) 'risk of bias'tool was used. ${ }^{13}$ Twelve items were scored as yes (= low risk), no (= high risk) or unclear. Review authors were not blinded for author names, institutions or journals. If additional information was needed, corresponding authors were contacted.

\section{DATA EXTRACTION AND DATA SYNTHESIS}

Data from the selected studies were extracted by one review author (RVE) and checked by a second review author $(\mathrm{IH})$. Extracted data included relevant reported information about the study population (age, gender, duration of symptoms, baseline score of outcomes), type of intervention (using an adapted version of the National Institutes of Health Behavior Change Consortium $(\mathrm{NIH}-$ BCC) checklist, Appendix 2), type of comparison intervention, type of methodology and analysis, and outcomes (baseline and follow-up). In case multiple publications existed regarding one $\mathrm{RCT}$, all available publications were checked and relevant data extracted. After data extraction from the included studies, it became evident that the studies were too heterogeneous to justify meta-analysis. Therefore, narrative analyses were conducted. The overall quality of the evidence was assessed using the Grades of Recommendation, Assessment, Development, and Evaluation (GRADE) approach. Evidence was defined as 'high quality evidence,', moderate quality evidence', 'low quality evidence', or 'very low quality evidence. ${ }^{17}$

\section{RESULTS}

The computer-aided database search identified 1,633 records (Figure 1). One additional record was identified through reference checking. After removing duplicates, 943 records remained and were screened on title and abstract. This resulted in 42 records to be assessed as full text for eligibility. Eventually, 7 RCTs (12 articles) met predefined eligibility criteria and were included in the systematic review. ${ }^{18-29}$ 
Figure 1 Study flow diagram

1,633 records identified trough database searching - MEDLINE $(n=376)$

- MEDLINE In-Process Citations \& Daily Update $(n=42$

- PubMed $(n=7)$

- Embase $(n=545)$

- PsyciNFO $(n=64)$

- $\operatorname{CINHAL}(n=238)$

- Cochrane Database of Systematic Reviews $(n=89)$

- CENTRAL $(n=171)$

- Database of Abstracts of Reviews of Effects $(n=6)$

- Health Technology Assessment (HTA) database $(n=5)$

- PEDro $(n=90)$

1 additional record identified

through other sources

691 duplicates removed

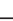

943 records screened on ti / ab.

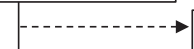

901 records excluded

42 records assessed on full -text

30 Full-text publications excluded:

- Not CLBP $(n=15)$

Not physiotherapist $(n=8)$

- Not primarycare $(n=3)$

- Not integrated BPS intervention $(n=1)$

- Not obtainable $(n=1)$

- No comparison intervention of interest $(n=2)$

$7 R C T$ s included in review

(12 records)

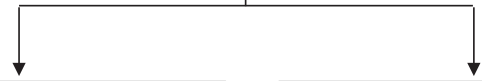

BPS THERAPY VS. ADVICE / GP CARE

McDonough 2013 (Clin J Pain)

Lamb 2010 (Lancet), 2012 (Pain), 2010 (HTA), 2010 (Osteopat),

Knox 2014 (BMC Musculoskelet Disord)

- Johnson 2007 (Spine)

BPS THERAPY VS. EXERCISE / MANUAL THERAPY / PHYSIOTHERAPY

Wälti 2015 (BMC Musculoskelet Disord)

- VibeFersum 2013 (Eur J Pain)

- Macedo 2012 (Phys Ther)

- vd Roer 2008 (Eur Spine J), 2008 (Spine) 


\section{CHARACTERISTICS OF INCLUDED STUDIES}

The studies included in the systematic review were all RCTs, written in English. Studies were conducted in Switzerland, ${ }^{29}$ Northern Ireland, ${ }^{25}$ Norway, $_{1}{ }^{28}$ Australia, ${ }_{1}^{24}$ the Netherlands, ${ }_{1}^{26}$ and the United Kingdom. ${ }^{18,21}$

\section{Participants}

Participants were recruited via general practitioners (GPs), medical specialists working in hospitals, primary care physiotherapists, retrospective searches (patient records) and advertisements. Sample sizes of the included studies ranged from 28 (pilot RCT) ${ }^{29}$ to 701 patients, ${ }^{21}$ resulting in an overall included sample size of 1,426 patients (Table 1). Mean baseline levels of disability and pain intensity were on average mild to moderate (Roland Morris Disability Questionnaire (RMDQ) range 9.0-12.1, Oswestry Disability Index (ODI) range 21.3-31.9, Pain Intensity Numerical Rating Scale (NRS) range 4.6-6.2, and Pain Intensity Visual Analogue Scale (VAS) range 45-59). Most studies reported an employment percentage between $47 \%$ and $72 \%$, except for Vibe Fersum et al. ${ }^{28}$ (88\% and 79\% in the biopsychosocial and control group, respectively). Three studies reported increased fear avoidance beliefs (Fear-Avoidance Beliefs Questionnaire: FABQ > 14) or kinesiophobia (Tampa Scale for Kinesiophobia:TSK $\left.\leq 37^{30}\right)$ ). ${ }^{21,25,26}$ In addition, Vibe Fersum et al. ${ }^{28}$ reported a relatively low FABQ score (FABQ physical activity $<14$ ). Wälti et al. ${ }^{29}$ reported a FABQ total score only $(23.93 \pm 11.58$ and $25.92 \pm 12.28)$. 


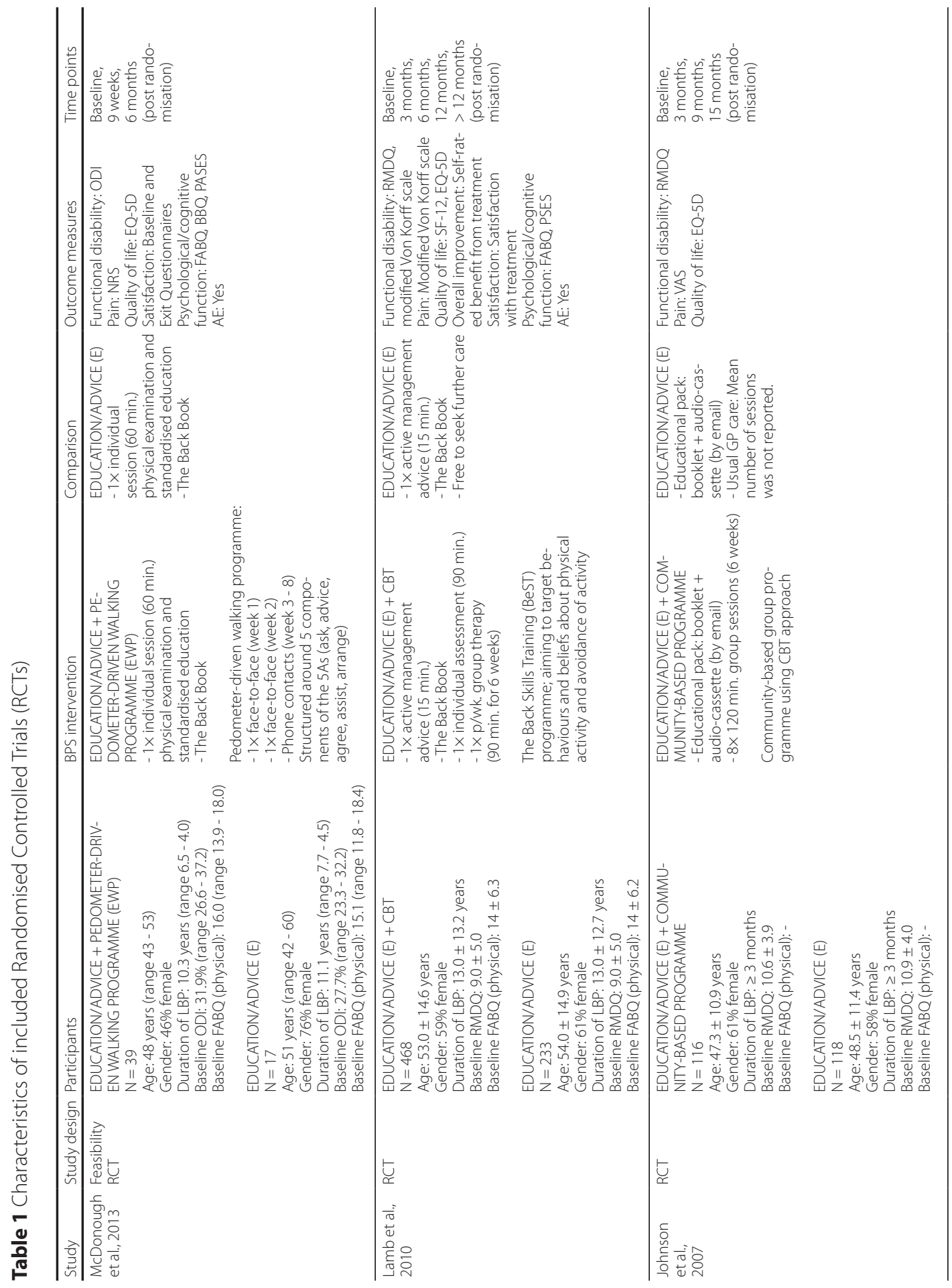




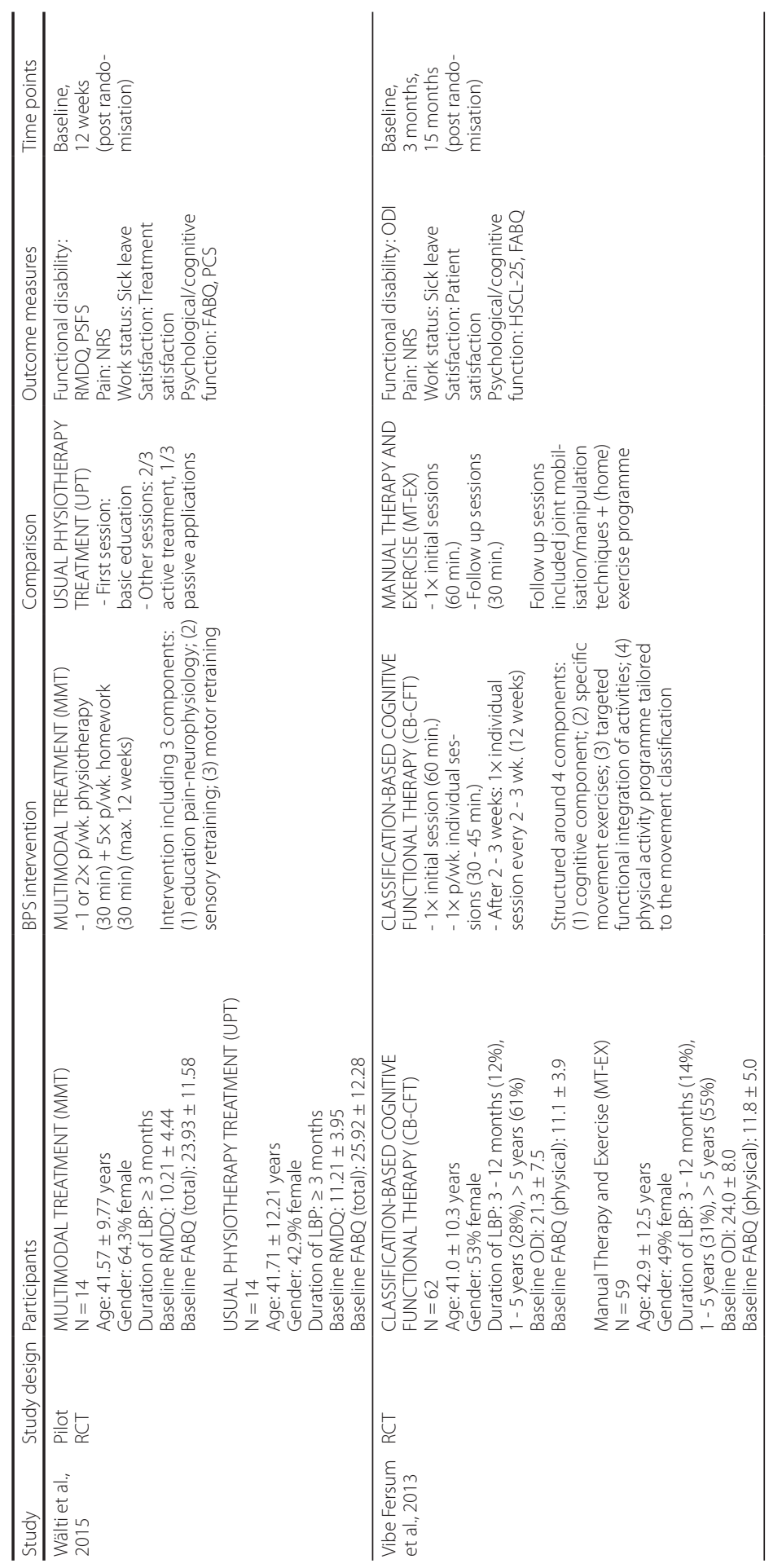




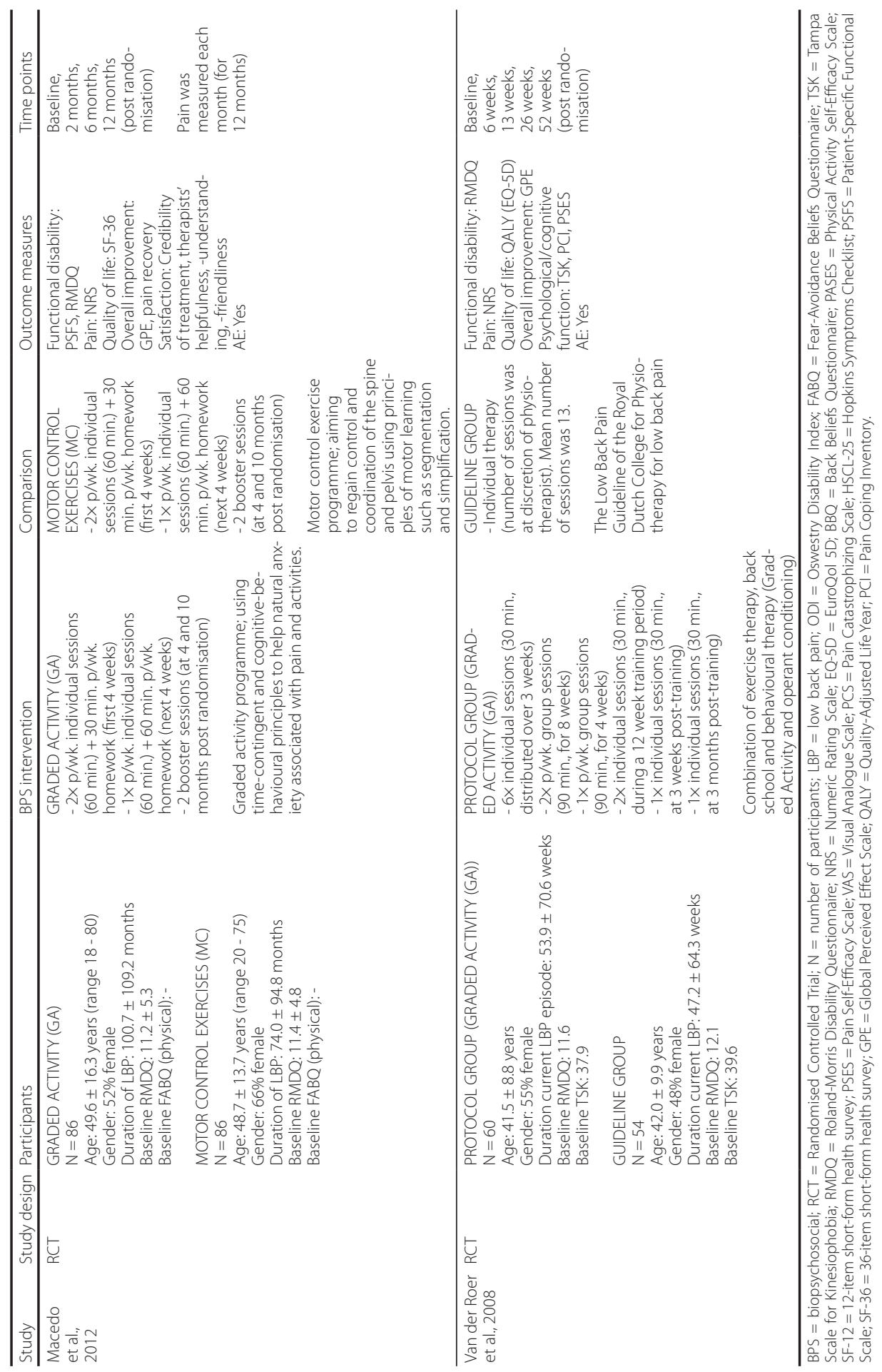




\section{Interventions}

Three studies compared a biopsychosocial intervention with education and advice. 18,21,25 Four studies compared a biopsychosocial intervention with a physical activity therapy. Physical activity therapy included usual/guideline physiotherapy, ${ }^{26,29}$ motor control therapy, ${ }^{24}$ and manual therapy plus exercise ${ }^{28}$ (Table 2).

The biopsychosocial interventions in all selected studies contained cognitive behavioural principles. However, the applied approach varied. Two studies used operant conditioning and Graded Activity principles, ${ }^{24,26}$ and another study used the 5A's model of health behaviour advice (ask/assess, advice, agree, assist, arrange). ${ }^{25}$ These three biopsychosocial interventions focused on specific exercise programmes to improve activity levels and cognitive-behavioural approaches were used additionally to encourage active behaviour. Another study used neurophysiological education about pain, disability and perceptions in addition to sensory and motor retraining. ${ }^{29}$ The remaining studies used cognitive behavioural therapy (CBT) $)^{18,21}$ or cognitive functional therapy. ${ }^{28}$ Although the latter two biopsychosocial interventions did include exercises, the main focus was on targeting beliefs and behaviour (e.g. to reduce fear avoidance and catastrophizing, and to improve coping style). By doing so, they aimed to stimulate/improve the level of functional activities. Four studies reported providing a booklet with education about LBP and coping strategies such as The Back Book, ${ }^{21,25}$ Explain Pain, ${ }^{29}$ or a general booklet on self-management strategies. ${ }^{18}$

All interventions were of low intensity ( $<30$ hours), except the biopsychosocial intervention of van der Roer et al. ${ }^{26}$ consisting of 35 hours contact time. The total duration of included biopsychosocial interventions ranged between 6 and 12 weeks. Two studies also provided booster sessions for the biopsychosocial interventions in the longer term (i.e. at 3 months, ${ }^{26}$ and at 4 and at 10 months $^{24}$ ). Four biopsychosocial interventions consisted of individual sessions, 24,25,28,29 two interventions of individual and group sessions combined, ${ }^{21,26}$ and one intervention of group sessions only. ${ }^{18}$ 


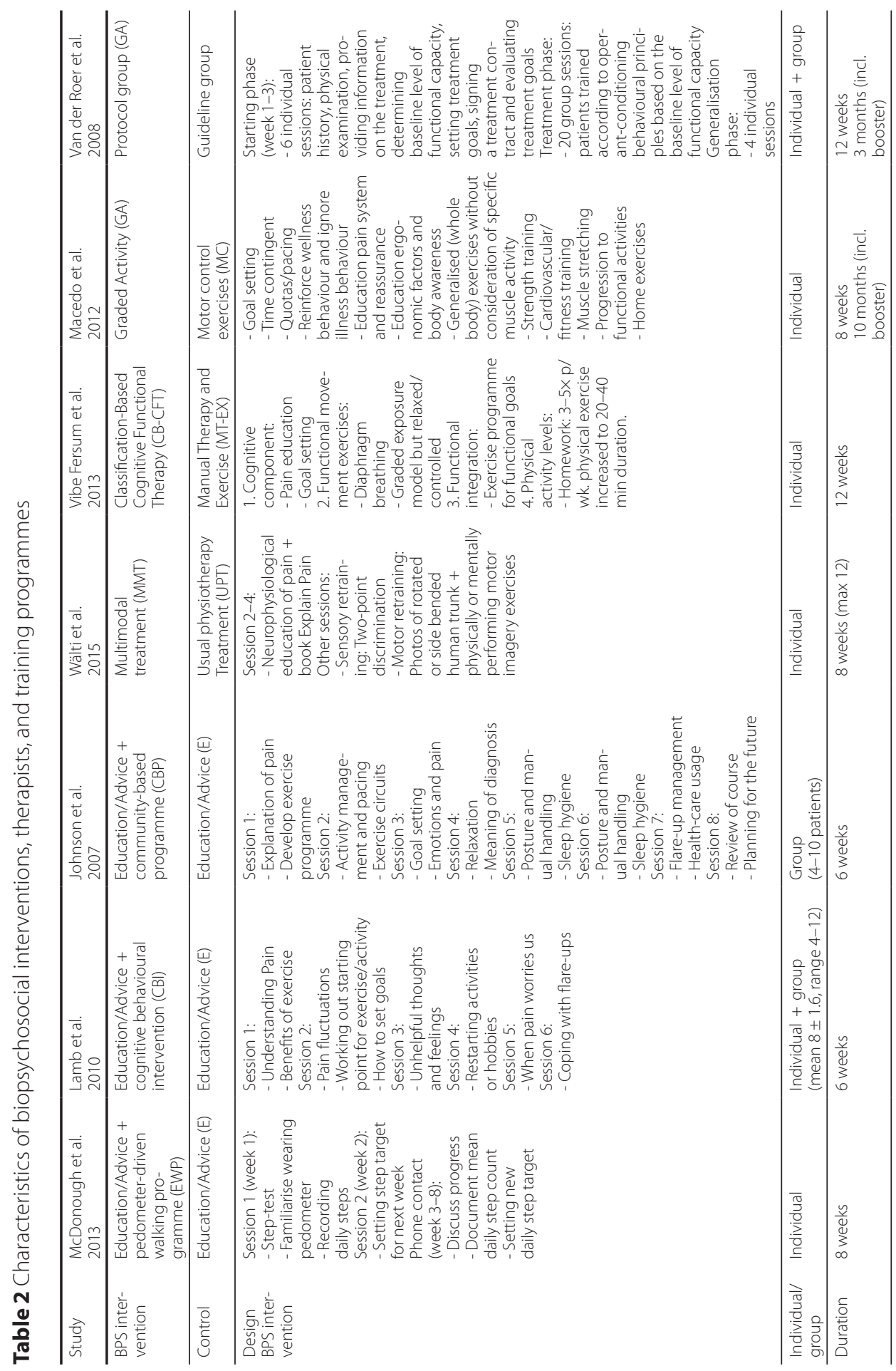




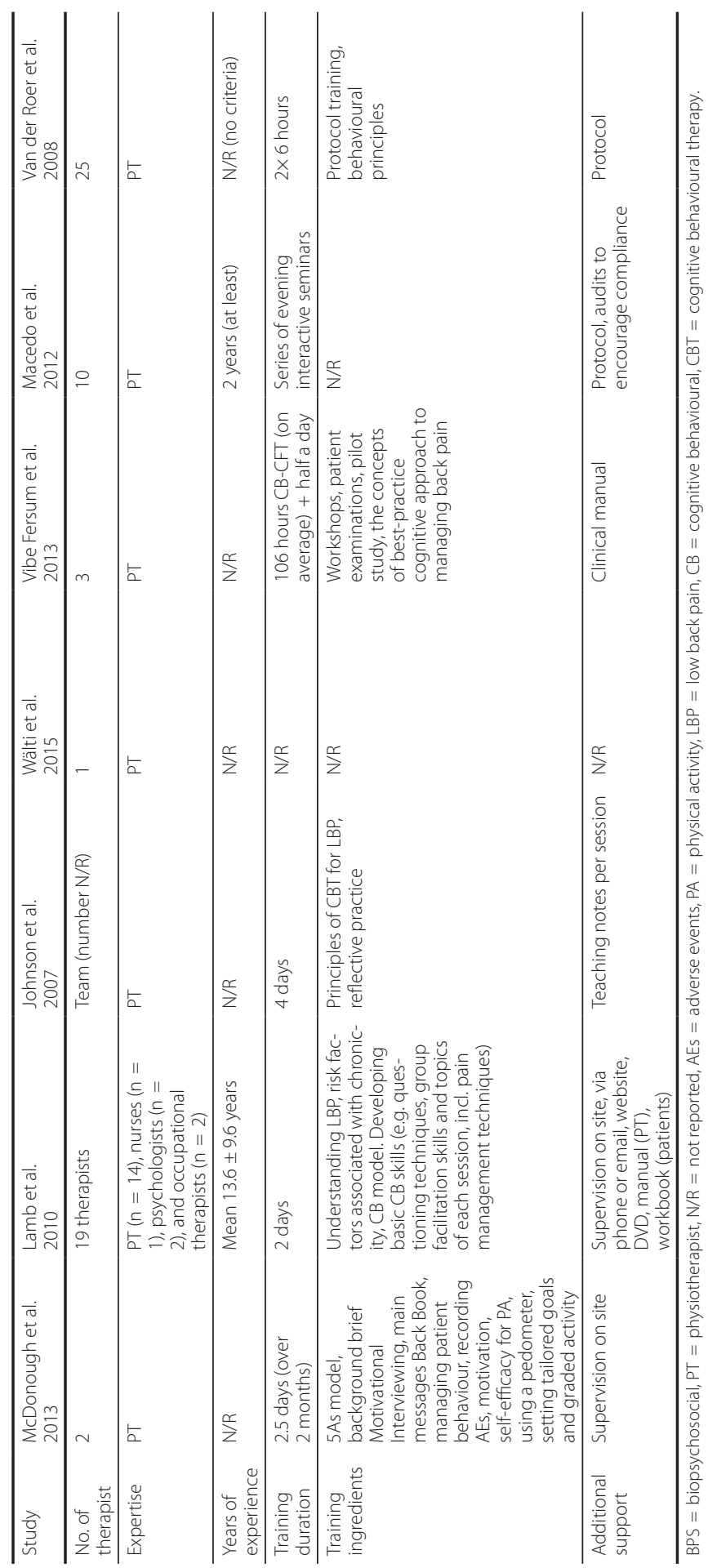




\section{Physiotherapists}

The number of physiotherapists providing the biopsychosocial interventions varied from one $\mathrm{e}^{29}$ or two, ${ }^{25}$ to $25^{26}$ (Table 2). Physiotherapists mostly received a short training programme with a duration ranging from 2 days ${ }^{21}$ to a maximum of 4 days..$^{18}$ One exception was Vibe Fersum et al. ${ }^{28}$ in which physiotherapists had on average 106 hours of cognitive behavioural training. Training programmes generally included protocol training and understanding of cognitive behavioural approaches. As additional support, most therapists received a protocol/manual. Lamb et al. ${ }^{21}$ and Vibe Fersum et al. ${ }^{28}$ were the only studies that provided (or at least reported on) support via other sources (Table 2).

\section{Outcomes}

All studies measured functional disability (RMDQ or ODI) and pain (NRS, Modified Von Korff Scale (MVKS) or VAS). Only four studies measured sick leave. ${ }^{21,26,28,29}$ Studies differed in the number and type of additional primary and secondary outcome measures (e.g. generic functional status, overall improvement, satisfaction, psychological and cognitive function, AEs). All studies provided data at short term ( $\leq 3$ months follow up), five studies at medium term ( $>3-12$ months follow-up), and five studies at long term ( $\geq 12$ months follow-up; all follow-up outcomes measured post-randomisation). Data at medium term were not available for Vibe Fersum et al. ${ }^{28}$ and Wälti et al. ${ }^{29}$ (pilot RCT), and not at long term for Wälti et al..$^{29}$ (pilot RCT) and McDonough at al. ${ }^{25}$ (feasibility RCT).

\section{RISK OF BIAS ASSESSMENT}

All studies reported an adequate method of randomisation (criterion 1) and 6 studies described treatment allocation as concealed (criterion 2; Table 3). The corresponding author of Johnson et al. ${ }^{18}$ replied that allocation in the study was not concealed for patients and therapists.

Six studies were not able to blind patients (criterion 3) and one study did not report on patient blinding..$^{29}$ All studies blinded neither therapists (criterion 4) nor outcome assessors (criterion 5), as therapists provided the treatments and therefore could not be blinded, and all studies used self-reported outcomes.

Only Vibe Fersum et al. ${ }^{28}$ showed incomplete outcome data ( $>20 \%{ }^{13}$ criterion 6). In this study, 16 of 59 patients (27.1\%) assigned to the control intervention and 11 of 62 patients (17.7\%) assigned to the biopsychosocial intervention were excluded from the analysis (due to not starting or not completing the interventions; total excluded 22.3\%). All studies had a low risk of selective outcome reporting (criterion 7). Furthermore, similar baseline characteristics (criterion 8) were reported in all except two studies. ${ }^{28,29}$ In Wälti et al. ${ }^{29}$, the percentage of females was higher in the biopsychosocial group than in the control group (64.3\% vs. $42.9 \%$ ) and pain catastrophizing (on the Pain Catastrophizing Scale (PCS)) was higher in the control group than in the biopsychosocial group (mean \pm SD $14.43 \pm 7.62$ vs. $20.08 \pm 8.24$ ). In Vibe Fersum et al. ${ }^{28}$ anxiety and depression 
(Hopkins Symptoms Checklist (HSCL)) and fear-avoidance of work (FABQ) were both higher in the control group than in the biopsychosocial group (mean \pm SD respectively $1.57 \pm 0.39$ vs. $1.40 \pm$ 0.33 , and $19.3 \pm 11.1$ vs. $14.1 \pm 9.6$ ). Studies did not report controlling for these variables.

Co-interventions were mostly neither reported nor clear (e.g. most studies did report trying to avoid co-interventions, but did not report the number or type of co-intervention, or whether they were similar between groups). Compliance with interventions (criterion 10) was acceptable in four studies, ${ }^{21,24,25,28}$ unclear in one study, ${ }^{26}$ and not acceptable in two studies. ${ }^{18,29}$ Wälti et al. ${ }^{29}$ reported good compliance in both interventions ( $>80 \%, \mathrm{n}=22$ ) but did not take into account the five patients who discontinued the intervention after 4 or more weeks of training, and the one patient who was lost to follow-up. Johnson et al. reported that non-compliance was modest (63\% attended at least half of the sessions) but that this influenced treatment effects.

All except two studies ${ }^{28,29}$ performed intention-to-treat analysis (criterion 11). Vibe Fersum et al. ${ }^{28}$ performed analysis on an 'available case basis' and Wälti et al. ${ }^{29}$ analysed 13 patients in the control group where 14 patients were originally included. Next, all studies reported similar timing of outcome assessment between intervention and control groups (criterion 12).

All included studies were RCTs, although Wälti et al. ${ }^{29}$ and McDonough et al. ${ }^{25}$ were a pilot and feasibility RCT, respectively. Wälti et al. ${ }^{29}$ aimed to evaluate the (short term) effects and feasibility of a multimodal intervention in order to calculate appropriate sample size for a larger RCT. The sample size was not calculated but pre-defined and small $(n=28)$. In addition, McDonough et al. ${ }^{25}$ was a feasibility RCT of a pedometer-driven walking programme (assessing recruitment, adherence, incidence AEs and effect sizes). For this study, no sample size (calculation) was reported and no significance tests were performed. The GRADE assessment of the quality of the evidence is presented in Table 4 and 5. 


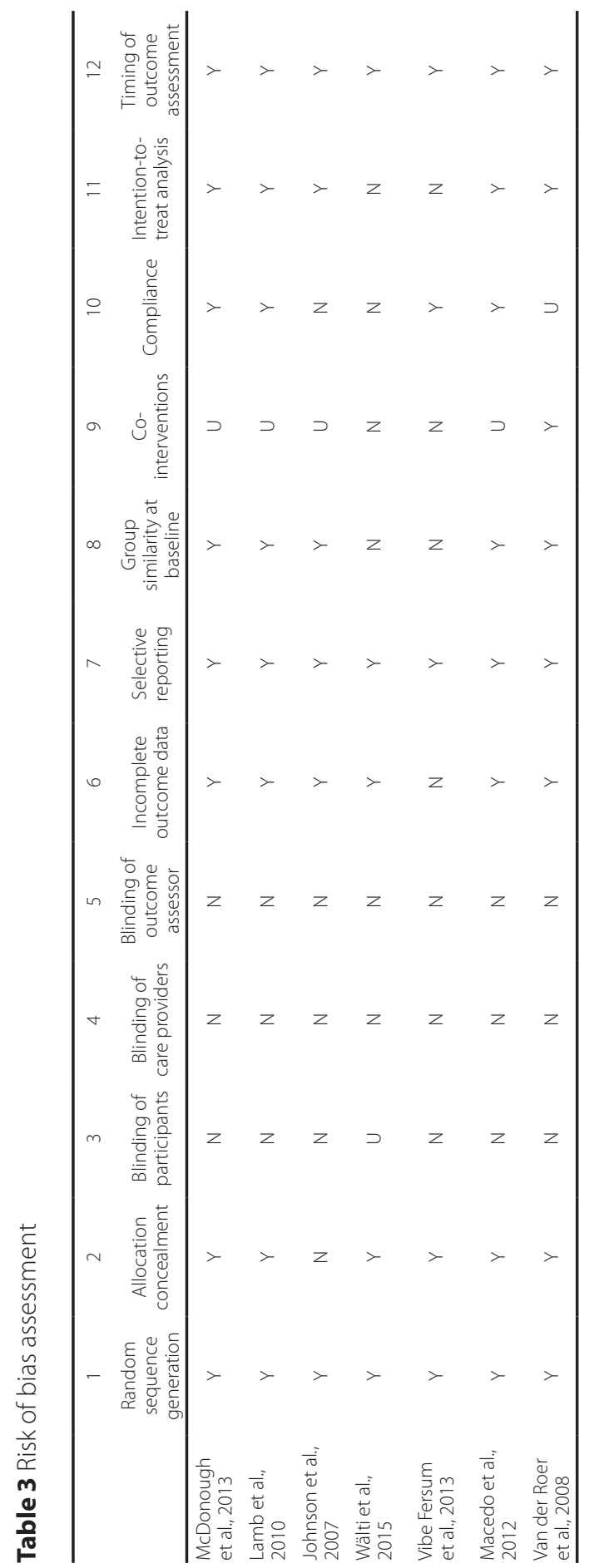


Table 4 GRADE evidence profile: biopsychosocial interventions compared to education and advice for patients with chronic low back pain

\begin{tabular}{|c|c|c|c|c|c|c|}
\hline \multicolumn{7}{|c|}{ Quality assessment } \\
\hline No of studies & Risk of bias & Inconsistency & Indirectness & Imprecision & Publication bias & $\begin{array}{c}\text { Overall quality } \\
\text { of evidence }\end{array}$ \\
\hline \multicolumn{7}{|c|}{ Functional disability level (follow up: mean 12 months; assessed with: RMDQ/ODI) } \\
\hline 3 RCTs & serious $^{a}$ & not serious & not serious & not serious & none & $\begin{array}{l}\oplus \oplus \oplus \bigcirc \\
\text { MODERATE }\end{array}$ \\
\hline \multicolumn{7}{|c|}{ Pain intensity (follow up: mean 12 months; assessed with:VAS/NRS) } \\
\hline 3 RCTs & serious $^{a}$ & not serious & not serious & not serious & none & $\begin{array}{l}\oplus \oplus \oplus \bigcirc \\
\text { MODERATE }\end{array}$ \\
\hline a. No blinding & & & & & & \\
\hline
\end{tabular}

Table 5 GRADE evidence profile: biopsychosocial interventions compared to physical activity interventions for patients with chronic low back pain

\begin{tabular}{|c|c|c|c|c|c|c|}
\hline \multicolumn{7}{|c|}{ Quality assessment } \\
\hline No of studies & Risk of bias & Inconsistency & Indirectness & Imprecision & Publication bias & $\begin{array}{l}\text { Overall quality } \\
\text { of evidence }\end{array}$ \\
\hline \multicolumn{7}{|c|}{ Functional disability level (follow up: mean 12 months; assessed with: RMDQ/ODI) } \\
\hline 4 RCTs & serious $^{a}$ & serious $^{b}$ & not serious & not serious & none & $\underset{\text { LOW }}{\oplus \oplus \bigcirc \bigcirc}$ \\
\hline \multicolumn{7}{|c|}{ Pain intensity (follow up: mean 12 months; assessed with:VAS/NRS) } \\
\hline 4 RCTs & serious $^{\text {a }}$ & serious $^{b}$ & not serious & not serious & none & $\underset{\text { LOW }}{\oplus \oplus \bigcirc}$ \\
\hline
\end{tabular}

\section{BIOPSYCHOSOCIAL INTERVENTION VERSUS EDUCATION/ADVICE}

\section{Primary outcomes (functional disability, pain, work status)}

At short term, the high quality RCT of Lamb et al..$^{21}$ showed significant between-group differences for functional disability and pain in favour of the biopsychosocial intervention (mean RMDQ 1.1 (95\% confidence interval (CI) 0.38 to 1.17); mean MVKS 4.2\%, (95\% Cl 0.40 to 8.10), mean MVKS pain $6.8 \%$ (95\% Cl 3.31 to 10.20), Appendix 3). The feasibility study of McDonough et al. ${ }^{25}$ also reported small but positive effects in favour of the biopsychosocial intervention, but reported effect sizes only (functional disability Cohen's $d=-0.39$, pain Cohen's $d=-0.10$ ) at short term. Johnson et al. ${ }^{18}$ did not find significant differences between the two interventions at short term. At medium and long term, all three studies found similar results for functional disability and pain. ${ }^{18,21,25}$ McDonough et al. ${ }^{25}$ additionally reported at 6 months (medium term) a higher percentage of patients achieving a minimal clinically important difference of $>10 \%$ in functional disability in the 
biopsychosocial group than in the control group (56\% vs. 44\%). For pain, no difference in clinically important improvements was visible between groups. For outcomes related to work status, only Lamb et al. ${ }^{21}$ measured sick leave and reported no differences between groups at all time points.

Secondary outcomes (quality of life, health status, psychological factors, AEs)

All three studies measured quality of life using the EuroQol-5D (EQ-5D) at all time points. ${ }^{18,21,25}$ At short term, mixed results were visible. Lamb et al. showed significant mean between-group difference of 0.07 ( $95 \%$ Cl 0.02 to 0.12) in favour of the biopsychosocial intervention, while McDonough et al. ${ }^{25}$ and Johnson et al. ${ }^{18}$ reported no effects and no significant between-group differences. At medium and long term follow-up, no studies found significant differences between the intervention groups. ${ }^{18,21,25}$ Lamb et al. ${ }^{21}$ also used the 12-item short-form health survey (SF-12) for quality of life. They found significant mean between-group differences for physical functioning scale at short, medium, and long term (e.g. long term -4.1 (95\% Cl -5.62 to -2.63)). The SF-12 was not used by McDonough et al. ${ }^{25}$ and Johnson et al..$^{18}$

Lamb et al. ${ }^{21}$ also showed significant larger mean improvements in fear-avoidance beliefs $(F A B Q)$ in the biopsychosocial intervention compared with the control intervention at all time points (short term 2.7 (95\% Cl 1.68 to 3.67); medium term 3.1 (95\% Cl 2.13 to 4.15); long term 2.9 (95\% Cl 1.83 to 4.03)). McDonough et al. ${ }^{25}$ also measured the FABQ at short- and medium term follow-up, but found no effect (Cohen's $d=-0.02$ and Cohen's $d=-0.06$, respectively). Johnson et al. ${ }^{18}$ did not measure fear-avoidance beliefs.

Only one AE (acute spinal cord compression with pulmonary embolus) was reported in the control group of Lamb et al..$^{21}$ which was not associated with the control intervention. McDonough et al..$^{25}$ reported 20 AEs in the biopsychosocial group of which 8 were related to the biopsychosocial intervention (increased pain in lower limb $(n=4)$ and back $(n=2)$, allergic reaction to metal clip of the pedometer which led to stopping the biopsychosocial intervention $(n=2)$. Johnson et al. ${ }^{18}$ did not report on AEs.

\section{BIOPSYCHOSOCIAL INTERVENTION VERSUS PHYSICAL ACTIVITY THERAPY}

\section{Primary outcomes (functional disability, pain, work status)}

One study with low methodological quality showed short term statistically and clinically important differences for functional disability in favour of the biopsychosocial intervention (mean ODI -9.7 (95\% Cl -12.7 to -6.7$)$ ). ${ }^{28}$ The differences remained significant at long term follow-up (mean ODI -8.2 (95\% Cl-12.6 to -3.8)). The other three studies did not find significant differences in functional disability between intervention groups and control groups at short-, 24,26,29 medium-, ${ }^{24,26}$ or long term follow-up. 24,26

For pain relief, mixed results were reported. At short term, the pilot study of Wälti et al. ${ }^{29}$ (mean NRS -1.45 (95\% Cl -4.0 to 0.0)) and Vibe Fersum et al. ${ }^{28}$ (mean NRS -2.1 (95\% Cl -2.7 to -1.4)) did find significant between-group differences in favour of the biopsychosocial intervention. The other two studies did not find significant between-group differences..$^{24,26}$ At medium term, two 
studies provided data. Macedo et al. ${ }^{24}$ did not find significant nor clinically important differences while van der Roer et al. ${ }^{26}$ found significant between-group differences in favour of the biopsychosocial interventions (mean NRS - 0.97 (95\% Cl -1.88 to -0.06)). At long term follow-up, three studies provided data for pain relief for which mixed results were found. ${ }^{24,26,29}$ Only Vibe Fersum et al. ${ }^{28}$ showed significant and clinically important improvements in favour of the biopsychosocial intervention (mean NRS -8.2 (95\% Cl -12.6 to -3.8)).

For outcomes related to work status, one study reported that sick leave did not occur during the study. ${ }^{29}$ Another study reported that patients in the biopsychosocial intervention had a 2.95 -times lower likelihood of taking sick. ${ }^{28}$ The other two studies either did not report ${ }^{24}$ or did not analyse sick leave. ${ }^{26}$

\section{Secondary outcomes (quality of life, health status, psychological factors, AEs)}

Only Macedo et al. ${ }^{24}$ measured quality of life and showed no between-group difference at short-, medium-, and long term follow-up (36-item short-form health survey (SF-36), physical and mental component). Van der Roer et al. ${ }^{26}$ performed an additional economic evaluation using the EQ-5D at long term follow-up, and reported no significant between-group differences (mean EQ-5D 0.03 (95\% Cl -0.06 to 0.12$)$ ).

Psychological factors were measured in three of the four studies. ${ }^{26,28,29}$ The FABQ was used in two studies of which one reported no differences in effects between groups (only measured at short term) ${ }^{29}$ and the other reported significant between-group differences in favour of the biopsychosocial intervention at short term (mean FABQ physical -3.6 (95\% Cl -5.3 to -1.9); mean FABQ work: -5.7 (95\% Cl -7.8 to -3.6$)$ ) and long term (mean FABQ physical -4.7 (95\% Cl -6.5 to -3.0); mean FABQ work -5.6 (95\% Cl -8.7 to -2.5)). ${ }^{28}$ Van der Roer et al. ${ }^{26}$ used other psychological functioning questionnaires (TSK; Pain Coping Inventory, PCl; Pain Self-Efficacy Scale, PSES) but could not identify significant differences between interventions, except for the mean PCI-P (items passive coping) at short term, which was in favour of the biopsychosocial intervention $(-0.61(95 \% \mathrm{Cl}-1.10$ to -0.12$))$.

Only one out of four studies reported AEs. ${ }^{24}$ They mentioned pain exacerbation $(n=35)$ and development of musculoskeletal complaints $(n=2)$ as similar in both intervention and control groups. Van der Roer et al. ${ }^{26}$ did not specifically report AEs but said there were no serious AEs.

\section{OUTCOMES RELATED TO TREATMENT DELIVERY}

Only one study reported most participating therapists having little or no experience in providing a biopsychosocial intervention. ${ }^{21}$ Three studies evaluated treatment delivery by physiotherapists. ${ }^{18,21,26}$ Lamb et al..$^{21}$ and Johnson et al. ${ }^{18}$ used audiotapes, and Van der Roer et al. ${ }^{26}$ used registration forms completed by physiotherapists (regarding therapy goals, content and evaluation of different therapy sessions), and regular therapy visits. Overall, Lamb et al. ${ }^{21}$ reported that therapists had satisfactorily delivered most of the predefined therapy items and satisfactorily demonstrated therapist skills (e.g. 100\% of the therapists listened appropriately, 63\% elicited beliefs/thoughts, and 77\% referred to the cognitive behavioural model). Johnson et al. ${ }^{18}$ reported 
that physiotherapists also delivered most cognitive behavioural components, but discovered that physiotherapists found it difficult to apply cognitive behavioural communication styles and to identify, for example, patients' anxieties and fears (73\% did not achieve this element). Van der Roer et al. ${ }^{26}$ noticed that therapists did not significantly increase their behavioural orientation after training, measured with the Pain Attitudes and Beliefs Scale for Physiotherapists (PABS-PT). This study reported that the protocol was adequately provided to $82 \%$ of the patients (i.e. treatment goals set, information provided, exercise scheme prepared). However, the extent to which cognitive behavioural skills were applied was not specifically assessed. The quality of the delivered cognitive behavioural components remains therefore unknown.

\section{OUTCOMES RELATED TO THE RECEIPT OF TREATMENT}

The percentage of patients who discontinued the biopsychosocial intervention when allocated and after treatment was started was low $(\leq 5 \%)$ in three studies, ${ }^{21,24,25}$ moderate $(\leq 21 \%)$ in three studies, ${ }^{26,28,29}$ and not reported in one study. ${ }^{18}$ Reasons for discontinuing from the biopsychosocial interventions include allergic reaction to pedometer $(7 \%){ }_{1}^{25}$ not able or willing to attend group sessions $(<1 \%),{ }^{21}$ losing person to support home exercises (7\%) or problems with online home training programme (14\%), ${ }^{29}$ time constraints (2\%), ${ }^{28}$ and not improving (1\% and 5\% respectively Macedo et al. ${ }^{24}$ and van der Roer et al. $\left.{ }^{26}\right)$. At the end, compliance was assessed differently in each study. As McDonough et al. ${ }^{25}$ evaluated a pedometer-driven walking programme, they assessed adherence to the step target. In total, $73 \%$ of the patients adhered to the weekly step target during the 8-week programme. Lamb et al. ${ }^{21}$ and Johnson et al. ${ }^{18}$ both reported that $63 \%$ attended at least half of the group sessions. Wälti et al..$^{29}$ assessed to what degree patients answered questions correctly and performed exercises. They reported that $\geq 81 \%$ reached the pre-defined adherence level. However, they did not take into account the data of six patients who dropped out.

\section{DISCUSSION}

This systematic review is, as far as we know, the first in comparing biopsychosocial primary care interventions delivered by physiotherapists with other primary care interventions for patients with CLBP. In addition, it provides an overview of theoretical and practical aspects of the biopsychosocial interventions included and is therefore useful for both researchers and clinicians. Our systematic review is based on seven studies, including one feasibility RCT and one pilot RCT, leaving five full-scale RCTs. Since the included studies suffered from methodological and/or practical limitations, new, higher quality studies would add valuable information to the findings of this systematic review.

In summary, this systematic review provides moderate quality evidence that a biopsychosocial intervention is more effective than education and advice in improving functional disability and pain at short, medium, and long term. For work status, no differences in effect were visible between the interventions. When a biopsychosocial intervention is compared to physical activity therapy, there is low quality evidence that both interventions are equally effective in improving functional disability, pain and work status at short, medium, and long term. 
From a practical point of view, nearly all biopsychosocial interventions included a physical component. Those biopsychosocial interventions who had an additional focus on psychosocial factors seemed especially promising. 18,21,28 Psychosocial factors included understanding pain, defining unhelpful thoughts/beliefs, avoidance behaviour, coping styles, and goal setting, Interventions with less focus on these elements (i.e. that did not focus as much or at all on psychosocial factors or self-defined functional activities that were valuable to the patient) seemed less effective. ${ }^{25,29}$

Training programmes were generally offered to physiotherapists to improve the delivery of biopsychosocial elements. While physiotherapists in the study of Vibe Fersum et al. ${ }^{28}$ were extensively trained, most studies offered short training programmes ranging from 2 to 4 days only. Van der Roer et al. ${ }^{26}$ reported that physiotherapists however did not significantly increase their behavioural orientation after a 2-day programme. Also physiotherapists in the study of Johnson et al. ${ }^{18}$ experienced difficulties when applying biopsychosocial principles in practice and discussing patients' beliefs and fears after a 4-day programme. Only the study of Lamb et al. ${ }^{21}$ reported adequate delivery after a short training programme. Physiotherapists had no or little experience prior to the start and received a 2-day training programme. These physiotherapists however received additionally a treatment protocol with a detailed description of each treatment session, supervision and a DVD with examples of the first sessions, and furthermore had access to a website with supporting materials. The extensive support in addition to the training programme could have positively influenced the delivery of the therapy.

The findings of the current systematic review are in part comparable to other systematic reviews. Kamper et al. ${ }^{4}$ for example reviewed the evidence for a multidisciplinary biopsychosocial intervention and also found moderate quality evidence for more effectiveness in pain relief and disability compared to usual care (GP). In line with the current systematic review, the systematic review of Kamper et al. ${ }^{4}$ found small differences in effects between intervention groups. The magnitude of change (i.e. between-group differences) required to meet the definition of clinical meaningfulness is debatable. It is mainly determined by the pros and cons of the therapy, or factors such as costs, effects on secondary outcomes, and convenience. ${ }^{31,32}$ In our systematic review, the biopsychosocial intervention was more favourable than education and advice when considering costs and psychological factors (e.g. fear-avoidance beliefs and self-efficacy ${ }^{21}$ ). No serious AEs were reported and also one study ${ }^{25}$ additionally reported a slightly higher number of patients achieving a minimal clinically important difference in the biopsychosocial intervention group (i.e. defined as a minimal improvement of 10\% points for ODI over time). Based on these findings, one might consider effects clinically meaningful and therefore consider a biopsychosocial intervention more promising than just education and advice.

Compared with physical treatments, Kamper et al. ${ }^{4}$ found low quality evidence for greater effectiveness of behavioural treatments, and Henschke et al. ${ }^{16}$ moderate quality evidence. Hall et al. ${ }^{9}$ even reported high-quality evidence for greater effectiveness of biopsychosocial interventions compared with exercise interventions. Our systematic review did not detect a difference between a biopsychosocial intervention and a physical activity therapy. The differences in findings are likely due to different therapy settings and participant populations included in previous mentioned systematic reviews. These systematic reviews focused on interventions delivered in both primary 
and secondary (multidisciplinary) care settings, and not in primary care settings specifically. Hall et al. ${ }^{9}$ furthermore did not focus on patients with CLBP in specific but rather on patients with acute, subacute, and chronic pain. Patients with CLBP might have different beliefs and behaviour, and might have received several treatments already. It is therefore possible that patients with a longer duration of LBP respond differently to treatments than patients with acute or subacute LBP.

Strengths of this systematic review are the search conducted by an experienced information specialist and the use of guidelines for optimal reporting. Furthermore, the systematic review included studies with RCT designs only. In general, RCTs represent the highest level of evidence as the risk of bias is lowest compared to other designs (e.g. observational designs). The main source of bias in the included studies was the inability to blind patients, therapists and outcome assessors. However, since patients may recognise the therapy to which they are allocated and therapists will know the intervention they are delivering, bias with respect to blinding is unavoidable.

A requirement of the systematic review was to only include studies with a biopsychosocial intervention of interest. Our definition of a biopsychosocial intervention was based on descriptions in current literature, self-defined criteria, and a previously-conducted systematic review. ${ }^{3,4}$ We defined a biopsychosocial intervention as a multicomponent intervention, focusing on biological (e.g. pain physiology, physical components), psychological (e.g. beliefs, behaviour, coping style), and social aspects (e.g. family, work, etc.). While studies did include these components in the biopsychosocial intervention, not every study provided in-depth information to what extent biopsychosocial elements were explicitly addressed during treatment. A protocol of the intervention may be helpful for a better interpretation and furthermore helpful for replication, implementation or development of a (new) biopsychosocial intervention. Other limitations were the heterogeneity in study and treatment designs and the use, or not, of measurement instruments, especially psychosocial ones. Two studies did not measure psychosocial factors at all. 18,24 Since biopsychosocial interventions initially aim to target psychosocial factors, psychosocial measurements are of high value and should be used in future trials.

Future clinical trials should develop and evaluate biopsychosocial primary care interventions that are specifically focused on functional goals (valuable for the patient) and psychosocial needs. It is important to select therapists based on their biopsychosocial skills or to adequately educate and support them in providing biopsychosocial elements. The essential role of sufficient training and resources (e.g. treatment protocols and support) in complex behavioural interventions can be confirmed by current literature. ${ }^{9,33}$ Both studies suggest that if physiotherapists receive appropriate training and resources, and possess sufficient competences, physiotherapists will be able to effectively provide a cognitive behavioural intervention. When reporting outcomes of trials, it is necessary to provide a detailed description of the treatment design (biopsychosocial elements), its practical implementation (process information), and information on the selection, training and supervision of physiotherapists. Such information is necessary for adequate interpretation of findings and for appropriate replication of biopsychosocial interventions in clinical practice. 


\section{CONCLUSIONS}

This systematic review shows beneficial effects for primary care biopsychosocial interventions over education and advice in patients with CLBP. Furthermore, primary care biopsychosocial interventions and physical activity interventions provide equally promising effects. However, some included studies suffered from methodological and practical limitations. In combination with the rather low number of studies evaluating primary care biopsychosocial interventions, it is recommended that additional studies of high methodological as well as practical quality be performed. This is of particular importance for comparison with physical activity treatments. High quality studies are expected to add valuable information to the findings of this systematic review and will be important for future directions of primary care interventions for patients with CLBP.

\section{ACKNOWLEDGEMENTS}

We acknowledge information specialist Steven Duffy for his contribution to performing the search strategy. Furthermore, we acknowledge Les Hearn for proofreading and editing the manuscript. 


\section{REFERENCES}

1. Merskey H, Bogduk N. Classification of Chronic Pain. Part III: Pain Terms, A Current List with Definitions and Notes on Usage. 1994:209-214.

2. Manchikanti L, Singh V, Falco FJ, Benyamin RM, Hirsch JA. Epidemiology of low back pain in adults. Neuromodulation. 2014;17 Suppl 2:3-10.

3. Waddell G. The back pain revolution. 2nd ed. ed. Oxford: Churchill Livingstone; 2004.

4. Kamper SJ, Apeldoorn AT, Chiarotto A, et al. Multidisciplinary biopsychosocial rehabilitation for chronic low back pain. The Cochrane database of systematic reviews. 2014;9:CD000963.

5. Lindstrom I, Ohlund C, Eek C, et al. The effect of graded activity on patients with subacute low back pain: a randomized prospective clinical study with an operant-conditioning behavioral approach. Physical therapy. 1992;72(4):279-290; discussion 291-273.

6. Vlaeyen JW, de Jong J, Geilen M, Heuts PH, van Breukelen G. Graded exposure in vivo in the treatment of pain-related fear: a replicated single-case experimental design in four patients with chronic low back pain. Behaviour research and therapy. 2001;39(2):151-166.

7. Trompetter HR, Bohlmeijer ET, Veehof MM, Schreurs KM. Internet-based guided self-help intervention for chronic pain based on Acceptance and Commitment Therapy: a randomized controlled trial. Journal of behavioral medicine. 2015;38(1):66-80.

8. Hayes SC. Acceptance and Commitment Therapy, Relational Frame Theory, and the Third Wave of Behavioral and Cognitive Therapies-Republished Article. Behav Ther. 2016;47(6):869-885.

9. Hall A, Richmond H, Copsey B, et al. Physiotherapist-delivered cognitive-behavioural interventions are effective for low back pain, but can they be replicated in clinical practice? A systematic review. Disability and rehabilitation. 2016:1-9.

10. Brunner E, De Herdt A, Minguet P, Baldew SS, Probst M. Can cognitive behavioural therapy based strategies be integrated into physiotherapy for the prevention of chronic low back pain? A systematic review. Disability and rehabilitation. 2013;35(1):1-10.

11. Ramond-Roquin A, Bouton C, Gobin-Tempereau AS, et al. Interventions focusing on psychosocial risk factors for patients with non-chronic low back pain in primary care-a systematic review. Family practice. 2014;31(4):379-388.

12. Synnott A, O'Keeffe M, Bunzli S, Dankaerts W, O'Sullivan P, O'Sullivan K. Physiotherapists may stigmatise or feel unprepared to treat people with low back pain and psychosocial factors that influence recovery: a systematic review. J Physiother. 2015;61(2):68-76.

13. Furlan AD, Malmivaara A, Chou R, et al. 2015 Updated Method Guideline for Systematic Reviews in the Cochrane Back and Neck Group. Spine. 2015;40(21):1660-1673.

14. Higgins JPT, Green S. Cochrane Handbook for Systematic Reviews of Interventions. 2011.

15. Liberati A, Altman DG, Tetzlaff J, et al. The PRISMA statement for reporting systematic reviews and meta-analyses of studies that evaluate healthcare interventions: explanation and elaboration. Bmj. 2009;339:b2700.

16. Henschke N, Ostelo RW, van Tulder MW, et al. Behavioural treatment for chronic low-back pain. The Cochrane database of systematic reviews. 2010(7):CD002014.

17. Guyatt GH, Oxman AD, Vist GE, et al. GRADE: an emerging consensus on rating quality of evidence and strength of recommendations. Bmj. 2008;336(7650):924-926. 
18. Johnson RE, Jones GT, Wiles NJ, et al. Active exercise, education, and cognitive behavioral therapy for persistent disabling low back pain: a randomized controlled trial. Spine. 2007;32(15):1578-1585.

19. Knox CR, Lall R, Hansen Z, Lamb SE. Treatment compliance and effectiveness of a cognitive behavioural intervention for low back pain: a complier average causal effect approach to the BeST data set. BMC musculoskeletal disorders. 2014;15:17.

20. Lamb SE, Hansen Z, Lall R, et al. Group cognitive behavioral intervention in primary care in low back pain: A randomized, controlled study and analysis of cost-effectiveness. [German]. Osteopathische Medizin. 2010;11(2):24-26.

21. Lamb SE, Hansen Z, Lall R, et al. Group cognitive behavioural treatment for low-back pain in primary care: a randomised controlled trial and cost-effectiveness analysis. Lancet. 2010;375(9718):916-923.

22. Lamb SE, Lall R, Hansen Z, et al. A multicentred randomised controlled trial of a primary care-based cognitive behavioural programme for low back pain. The Back Skills Training (BeST) trial. Health Technol Assess. 2010;14(41):1-253, iii-iv.

23. Lamb SE, Mistry D, Lall R, et al. Group cognitive behavioural interventions for low back pain in primary care: extended follow-up of the Back Skills Training Trial (ISRCTN54717854). Pain. 2012;153(2):494-501.

24. Macedo LG, Latimer J, Maher CG, et al. Effect of motor control exercises versus graded activity in patients with chronic nonspecific low back pain: a randomized controlled trial. Physical therapy. 2012;92(3):363377.

25. McDonough SM, Tully MA, Boyd A, et al. Pedometer-driven walking for chronic low back pain: a feasibility randomized controlled trial. The Clinical journal of pain. 2013;29(11):972-981.

26. van der Roer N, van Tulder M, Barendse J, Knol D, van Mechelen W, de Vet H. Intensive group training protocol versus guideline physiotherapy for patients with chronic low back pain: a randomised controlled trial. European spine journal : official publication of the European Spine Society, the European Spinal Deformity Society, and the European Section of the Cervical Spine Research Society. 2008;17(9):1193-1200.

27. van der Roer $N$, van Tulder M, van Mechelen W, de Vet H. Economic evaluation of an intensive group training protocol compared with usual care physiotherapy in patients with chronic low back pain. Spine. 2008;33(4):445-451.

28. Vibe Fersum K, O'Sullivan P, Skouen JS, Smith A, Kvale A. Efficacy of classification-based cognitive functional therapy in patients with non-specific chronic low back pain: a randomized controlled trial. European journal of pain. 2013;17(6):916-928.

29. Walti P, Kool J, Luomajoki H. Short-term effect on pain and function of neurophysiological education and sensorimotor retraining compared to usual physiotherapy in patients with chronic or recurrent non-specific low back pain, a pilot randomized controlled trial. BMC musculoskeletal disorders. 2015;16:83.

30. Vlaeyen JW, Kole-Snijders AM, Boeren RG, van Eek H. Fear of movement/(re)injury in chronic low back pain and its relation to behavioral performance. Pain. 1995;62(3):363-372.

31. Dworkin RH, Turk DC, McDermott MP, et al. Interpreting the clinical importance of group differences in chronic pain clinical trials: IMMPACT recommendations. Pain. 2009;146(3):238-244.

32. Dworkin RH, Turk DC, Wyrwich KW, et al. Interpreting the clinical importance of treatment outcomes in chronic pain clinical trials: IMMPACT recommendations. The journal of pain: official journal of the American Pain Society. 2008;9(2):105-121.

33. van der Windt D, Hay E, Jellema P, Main C. Psychosocial interventions for low back pain in primary care: lessons learned from recent trials. Spine. 2008;33(1):81-89. 


\section{APPENDIX}

\section{APPENDIX I SEARCH STRATEGIES}

Medline (Ovid): 1946-2015/Nov week 2

Date searched: 30.11 .15

1 Low Back Pain/ (15806)

2 exp Back Pain/ and Chronic Disease/ (3314)

3 ((low or lower or lumbal or lumbosacral or lumbo-sacral or lumbar\$) adj3 (back pain\$ or backache\$ or back-ache\$ or backpain\$)).ti,ab. (18719)

4 ((low back or lower back or lowback or lowerback or loin) adj3 (pain or pains or ache\$ or painful\$ or pained or hurt or hurts or hurting or sore or soreness or tender\$ or discomfort or aching or agony)).ti,ab. (19309)

5 ((lumbal or lumbar\$ or lumbosacral or lumbo-sacral or lumbosacroiliac or lumbo sacroiliac or spine or spinal) adj3 (pain or pains or painful\$ or pained or hurt or hurts or hurting or sore or soreness or tender\$ or discomfort or aching or agony or ache\$ or backache\$ or backpain\$)).ti,ab. (7304)

6 ((lumbar\$ or lumbal or lumbosacral or lumbo-sacral) adj2 syndrome\$).ti,ab. (635)

7 (lumbago or lumbalgesia or lumbalgia or lumbodynia).ti,ab. (1234)

8 or/1-7 (32285)

$9 \exp$ Behavior Therapy/ (57760)

10 exp "Conditioning (Psychology)"/ (50253)

11 ((behavio?r\$ or cognitive or implosive or implosion or aversion) adj3 (therap\$ or treatment\$ or training or modify or modification or modified)).ti,ab. (41306)

12 desensiti?ation.ti,ab. (20137)

13 CBT.ti,ab. (5382)

14 (conditioning or conditional stimul\$ or conditioned behavio?r\$ or operant treatment\$ or operant behavio?r\$).ti,ab. (46027)

15 Patient Education as Topic/ (74402)

16 ((patient\$ or consumer\$ or health) adj3 (educat\$ or learn\$ or teach\$ or train\$)).ti,ab. (87565)

17 psychoeducat\$.ti,ab. (2681)

18 (graded adj3 (activit\$ or exposure\$)).ti,ab. (709)

19 exp Self Care/ (44305)

20 (self care or self regulat\$ or self manag\$ or self monitor\$ or self help).ti,ab. (34119)

21 exp Physical Therapy Modalities/ (135645)

22 Physical Therapists/ (563)

23 (physiotherap\$ or physio therap\$ or (physical adj2 therap\$)).ti,ab. (29211)

24 ((psychosocial or psycho social or bio psychosocial or bio psycho social or biopsycho social) adj3 (care\$ or intervention\$ or therap\$ or treatment\$ or support\$ or rehab\$)).ti,ab. (11148)

25 or/9-24 (519296)

26 exp Primary Health Care/ (87766) 
27 (primary care or primary health care or primary health service\$ or primary healthcare or primary medical care).ti,ab. (86844)

28 or/26-27 (134704)

298 and 25 and 28 (376)

30 exp Animals/ not (exp Animals/ and Humans/) (4150916)

3129 not $30(376)$

Medline In-Process \& Other Non-Indexed Citations (Ovid); Medline Daily Update (Ovid): November 242015

Date searched: 30.11 .15

1 Low Back Pain/ (16)

2 exp Back Pain/ and Chronic Disease/ (0)

3 ((low or lower or lumbal or lumbosacral or lumbo-sacral or lumbar\$) adj3 (back pain\$ or backache\$ or back-ache\$ or backpain\$)).ti,ab. (2661)

4 ((low back or lower back or lowback or lowerback or loin) adj3 (pain or pains or ache\$ or painful\$ or pained or hurt or hurts or hurting or sore or soreness or tender\$ or discomfort or aching or agony)).ti,ab. (2785)

5 ((lumbal or lumbar\$ or lumbosacral or lumbo-sacral or lumbosacroiliac or lumbo sacroiliac or spine or spinal) adj3 (pain or pains or painful\$ or pained or hurt or hurts or hurting or sore or soreness or tender\$ or discomfort or aching or agony or ache\$ or backache\$ or backpain\$)).ti,ab. (975)

6 ((lumbar\$ or lumbal or lumbosacral or lumbo-sacral) adj2 syndrome\$).ti,ab. (48)

7 (lumbago or lumbalgesia or lumbalgia or lumbodynia).ti,ab. (65)

8 or/1-7 (3664)

9 exp Behavior Therapy/ (56)

10 exp "Conditioning (Psychology)"/ (19)

11 ((behavio? $\$$ or cognitive or implosive or implosion or aversion) adj3 (therap\$ or treatment\$ or training or modify or modification or modified)).ti,ab. (5024)

12 desensiti?ation.ti,ab. (714)

13 CBT.ti,ab. (865)

14 (conditioning or conditional stimul\$ or conditioned behavio?r\$ or operant treatment\$ or operant behavio?r\$).ti,ab. (3636)

15 Patient Education as Topic/ (43)

16 ((patient\$ or consumer\$ or health) adj3 (educat\$ or learn\$ or teach\$ or train\$)).ti,ab. (9525)

17 psychoeducat\$.ti,ab. (400)

18 (graded adj3 (activit\$ or exposure\$)).ti,ab. (42)

19 exp Self Care/ (61)

20 (self care or self regulat\$ or self manag\$ or self monitor\$ or self help).ti,ab. (4503)

21 exp Physical Therapy Modalities/ (171)

22 Physical Therapists/ (4)

23 (physiotherap\$ or physio therap\$ or (physical adj2 therap\$)).ti,ab. (4015) 
24 ((psychosocial or psycho social or bio psychosocial or bio psycho social or biopsycho social) adj3 (care\$ or intervention\$ or therap\$ or treatment\$ or support\$ or rehab\$)).ti,ab. (1241)

25 or/9-24 (27887)

26 exp Primary Health Care/ (87)

27 (primary care or primary health care or primary health service\$ or primary healthcare or primary medical care).ti,ab. (9510)

28 or/26-27 (9548)

298 and 25 and 28 (42)

30 exp Animals/ not (exp Animals/ and Humans/) (2036)

3129 not $30(42)$

PubMed (NLM): up to 30 November 2015

Date searched: 30.11 .15

\#18 Search \#16 AND\#17 7

\#17 Search pubstatusaheadofprint OR publisher[sb] (491894)

\#16 Search (\#4 AND \#14 AND \#15) (271)

\#15 Search "primary care"[tiab] OR "primary health care"[tiab] OR "primary health service"[tiab OR "primary health services"[tiab] OR "primary healthcare" OR "primary medical care" (100170)

\#14 Search (\#5 OR \#6 OR \#7 OR \#8 OR \#9 OR \#10 OR \#11 OR \#12 OR \#13) (483699)

\#13 search (psychosocial[tiab] OR "psycho social "[tiab] OR "bio psychosocial"[tiab] OR "bio psycho social"[tiab] OR "biopsycho social"[tiab]) AND (care*[tiab] OR intervention*[tiab] OR therap*[tiab] OR treatment*[tiab] OR support*[tiab] OR rehab*[tiab]) (49587)

\#12 Search physiotherapy*[tiab] OR "physio therapy"[tiab] OR "physical therapy"[tiab] OR "physio therapist"[tiab] OR "physical therapist"[tiab] OR "physio therapists"[tiab] OR "physical therapists"[tiab] (29835)

\#11 Search "self care"[tiab] OR "self regulation"[tiab] OR "self regulate"[tiab] OR "self manage"[tiab] OR "self management"[tiab] OR "self monitor"[tiab] OR "self monitoring"[tiab] OR "self help"[tiab] (36396)

\#10 Search "graded activity"[tiab] OR "graded activities"[tiab] OR "graded exposure"[tiab] OR "graded exposures"[tiab] (283)

\#9 Search psychoeducat*[tiab] (3158)

\#8 Search "patient education"[tiab] OR "consumer education"[tiab] OR "health education"[tiab] OR "client education"[tiab] (38816)

\#7 Search conditioning[tiab] OR "conditional stimulation "[tiab] OR "conditioned behavior"[tiab] OR "conditional behavior "[tiab] OR "operant treatment "[tiab] OR "operant behavior"[tiab] OR "operant behaviour "[tiab] (949529)

\#6 Search desensitisation[tiab] OR desensitization[tiab] OR CBT[tiab] (27307)

\#5 Search (behaviour*[tiab] OR behavior*[tiab] OR cognitive[tiab] OR implosive[tiab] OR implosion[tiab] OR aversion[tiab]) AND (therap*[tiab] OR treatment*[tiab] OR training[tiab] OR modify[tiab] OR modification[tiab] OR modified[tiab]) (281514)

\#4 Search (\#1 OR \#2 OR \#3) (27595) 
\#3 Search lumbago[tiab] OR lumbalgesia[tiab] OR lumbalgia[tiab] OR lumbodynia[tiab] (1350)

\#2 Search (lumbar*[tiab] OR lumbal[tiab] OR lumbosacral[tiab] OR lumbo-sacral[tiab]) AND syndrome*[tiab] (5771)

\#1 Search ("low back pain"[tiab] OR "lower back pain"[tiab] OR "lumbal back pain"[tiab] OR "lumbosacral back pain"[tiab] OR "lumbo-sacral back pain"[tiab] OR "lumbar back pain"[tiab] OR "low backache"[tiab] OR "lower backache"[tiab] OR "lumbal backache"[tiab] OR "lumbosacral backache"[tiab] OR "lumbo-sacral backache"[tiab] OR "lumbar backache"[tiab] OR "low backpain"[tiab] OR "lower backpain"[tiab] OR "lumbal backpain"[tiab] OR "lumbosacral backpain"[tiab] OR "lumbo-sacral backpain"[tiab] OR "lumbar backpain"[tiab]) (21196)

Embase (Ovid): 1974-2015/week 47

Date searched: 30.11 .15

1 low back pain/ (40687)

2 ((low or lower or lumbal or lumbosacral or lumbo-sacral or lumbar\$) adj3 (back pain\$ or backache\$ or back-ache\$ or backpain\$)).ti,ab. (28062)

3 ((low back or lower back or lowback or lowerback or loin) adj3 (pain or pains or ache\$ or painful\$ or pained or hurt or hurts or hurting or sore or soreness or tender\$ or discomfort or aching or agony)).ti,ab. (29026)

4 (lumbal or lumbar\$ or lumbosacral or lumbo-sacral or lumbosacroiliac or lumbo sacroiliac or spine or spinal) adj3 (pain or pains or painful\$ or pained or hurt or hurts or hurting or sore or soreness or tender\$ or discomfort or aching or agony or ache\$ or backache\$ or backpain\$)).ti,ab. (11682)

5 ((lumbar\$ or lumbal or lumbosacral or lumbo-sacral) adj2 syndrome\$).ti,ab. (923)

6 (lumbago or lumbalgesia or lumbalgia or lumbodynia).ti,ab. (1833)

7 or/1-6 (56318)

8 behavior therapy/ (39189)

9 exp cognitive therapy/ (39468)

10 exp conditioning/ (58564)

11 ((behavio? $\$$ or cognitive or implosive or implosion or aversion) adj3 (therap\$ or treatment\$ or training or modify or modification or modified)).ti,ab. (64702)

12 desensiti?ation.ti,ab. (25146)

13 CBT.ti,ab. (9563)

14 (conditioning or conditional stimul\$ or conditioned behavio?r\$ or operant treatment\$ or operant behavio?r\$).ti,ab. (67931)

15 patient education/ (93209)

16 ((patient\$ or consumer\$ or health) adj3 (educat\$ or learn\$ or teach\$ or train\$)).ti,ab. (129474)

17 psychoeducat\$.ti,ab. (4692)

18 (graded adj3 (activit\$ or exposure\$)).ti,ab. (957)

19 exp self care/ (56960)

20 (self care or self regulat\$ or self manag\$ or self monitor\$ or self help).ti,ab. (51035) 
21 exp physiotherapy/ (62877)

22 physiotherapist/ (11756)

23 (physiotherap\$ or physio therap\$ or (physical adj2 therap\$)).ti,ab. (51334)

24 psychosocial care/ (13110)

25 ((psychosocial or psycho social or bio psychosocial or bio psycho social or biopsycho social) adj3 (care\$ or intervention\$ or therap\$ or treatment\$ or support\$ or rehab\$)).ti,ab. (17781)

26 or/8-25 (587936)

27 exp primary health care/ (121229)

28 (primary care or primary health care or primary health service\$ or primary healthcare or primary medical care).ti,ab. (121033)

29 or/27-28 (164101)

307 and 26 and 29 (545)

31 animal/ (1707432)

32 animal experiment/ (1897167)

33 (rat or rats or mouse or mice or murine or rodent or rodents or hamster or hamsters or pig or pigs or porcine or rabbit or rabbits or animal or animals or dogs or dog or cats or cow or bovine or sheep or ovine or monkey or monkeys).ti,ab,ot,hw. (6089240)

34 or/31-33 (6089240)

35 exp human/ or human experiment/ (16579563)

3634 not (34 and 35) (4804905)

3730 not $36(545)$

PsycINFO (Ovid): 1806-2015/Nov week 3

Date searched: 30.11 .15

1 back pain/ (3131)

2 ((low or lower or lumbal or lumbosacral or lumbo-sacral or lumbar\$) adj3 (back pain\$ or backache\$ or back-ache\$ or backpain\$)).ti,ab. (2851)

3 ((low back or lower back or lowback or lowerback or loin) adj3 (pain or pains or ache\$ or painful\$ or pained or hurt or hurts or hurting or sore or soreness or tender\$ or discomfort or aching or agony)).ti,ab. (2914)

4 (lumbal or lumbar\$ or lumbosacral or lumbo-sacral or lumbosacroiliac or lumbo sacroiliac or spine or spinal) adj3 (pain or pains or painful\$ or pained or hurt or hurts or hurting or sore or soreness or tender\$ or discomfort or aching or agony or ache\$ or backache\$ or backpain\$)).ti,ab. (934)

5 ((lumbar\$ or lumbal or lumbosacral or lumbo-sacral) adj2 syndrome\$).ti,ab. (22)

6 (lumbago or lumbalgesia or lumbalgia or lumbodynia).ti,ab. (31)

7 or/1-6 (4720)

8 biopsychosocial approach/ (3107)

9 exp behavior therapy/ (17758)

10 exp conditioning/ (50205)

11 cognitive therapy/ (12175) 
12 ((behavio?r\$ or cognitive or implosive or implosion or aversion) adj3 (therap\$ or treatment\$ or training or modify or modification or modified)).ti,ab. (65161)

13 desensiti?ation.ti,ab. (5366)

14 CBT.ti,ab. (9242)

15 (conditioning or conditional stimul\$ or conditioned behavio?r\$ or operant treatment\$ or operant behavio?\$).ti,ab. (32339)

16 client education/ (3282)

17 ((patient\$ or consumer\$ or health or client\$) adj3 (educat\$ or learn\$ or teach\$ or train\$)). ti,ab. (37050)

18 psychoeducation/ (3546)

19 psychoeducat\$.ti,ab. (6828)

20 (graded adj3 (activit\$ or exposure\$)).ti,ab. (278)

21 self care skills/ (3665)

22 (self care or self regulat\$ or self manag\$ or self monitor\$ or self help).ti,ab. (38145)

23 physical therapy/ (2119)

24 physical therapists/ (369)

25 (physiotherap\$ or physio therap\$ or (physical adj2 therap\$)).ti,ab. (5025)

26 ((psychosocial or psycho social or bio psychosocial or bio psycho social or biopsycho social) adj3 (care\$ or intervention\$ or therap\$ or treatment\$ or support\$ or rehab\$)).ti,ab. (12393)

27 or/8-26 (236175)

28 primary health care/ (14121)

29 (primary care or primary health care or primary health service\$ or primary healthcare or primary medical care).ti,ab. (25365)

30 or/28-29 (27477)

317 and 27 and 30 (64)

CINAHL (EBSCO): 1982-20151201

Date searched: 1.12 .15

S28 S8 AND S24 AND S27 (238)

S27 S25 OR S26 (52,023)

S26 "primary care" or "primary health care" or "primary health service*" or "primary healthcare" or "primary medical care" $(52,023)$

S25 (MH "Primary Health Care") $(32,401)$

S24 S9 OR S10 OR S11 OR S12 OR S13 OR S14 OR S15 OR S16 OR S17 OR S18 OR S19 OR S20 OR S21 OR S22 OR S23 $(306,853)$

S23 (psychosocial or "psycho social" or "bio psychosocial" or "bio psycho social" or "biopsycho social") N3 (care* or intervention* or therap* or treatment* or support* or rehab*) $(59,463)$

S22 physiotherap* or "physio therap*" or (physical N2 therap*) $(45,897)$

S21 (MH "Physical Therapists") $(6,963)$

S20 (MH "Physical Therapy+") $(75,208)$

S19 "self care" or "self regulat*" or "self manag*" or "self monitor*" or "self help" $(34,361)$

S18 (MH "Self Care+") (26,816) 
S17 graded N3 (activit* or exposure*) (135)

S16 psychoeducat* $(2,328)$

S15 (patient* or consumer* or health) N3 (educat* or learn* or teach* or train*) $(107,589)$

S14 (MH "Patient Education+") $(49,596)$

S13 conditioning or "conditional stimul*" or "conditioned behavio\#r*" or "operant treatment*" or "operant behavio\#r*" $(3,747)$

S12 desensitisation or desensitization or CBT $(3,078)$

S11 (behavior* or behaviour* or cognitive or implosive or implosion or aversion) N3 (therap* or treatment* or training or modify or modification or modified) $(22,845)$

S10 (MH "Conditioning (Psychology)") (514)

S9 (MH "Behavior Therapy+") (13,423)

S8 S1 OR S2 OR S3 OR S4 OR S5 OR S6 OR S7 $(14,886)$

S7 lumbago or lumbalgesia or lumbalgia or lumbodynia (45)

S6 (lumbar* or lumbal or lumbosacral or lumbo-sacral) N2 syndrome* (90)

S5 (lumbal or lumbar* or lumbosacral or lumbo-sacral or lumbosacroiliac or lumbo sacroiliac or spine or spinal) N3 (pain or pains or painful* or pained or hurt or hurts or hurting or sore or soreness or tender* or discomfort or aching or agony or ache* or backache* or backpain*) $(2,499)$

S4 ("low back" or "lower back" or lowback or lowerback or loin) N3 (pain or pains or ache* or painful* or pained or hurt or hurts or hurting or sore or soreness or tender* or discomfort or aching or agony) $(12,532)$

S3 (low or lower or lumbal or lumbosacral or lumbo-sacral or lumbar*) N3 ("back pain*" or backache* or back-ache* or backpain*) $(12,462)$

S2 (MH "Chronic Pain") AND (MH "Back Pain + ") (2,213)

S1 (MH "Low Back Pain") (10,349)

Cochrane Database of Systematic Reviews (CDSR) (Wiley). Issue 12 of 12: December 2015

Cochrane Central Register of Controlled Trials (CENTRAL) (Wiley). Issue 11 of 12: November 2015

Database of Abstracts of Reviews of Effects (DARE) (Wiley). Issue 2 of 4: April 2015

Health Technology Assessment database (HTA) (Wiley). Issue 4 of 4: October 2015

Date searched: 1.12.15

\#1 [mh ^ "Low Back Pain"] (177)

\#2 [mh "Back Pain"] and [mh ^^"Chronic Disease"] (624)

\#3 (low or lower or lumbal or lumbosacral or lumbo-sacral or lumbar*) near/3 (back pain* or backache* or back-ache* or backpain*) (5554)

\#4 ("low back" or "lower back" or lowback or lowerback or loin) near/3 (pain or pains or ache* or painful* or pained or hurt or hurts or hurting or sore or soreness or tender* or discomfort or aching or agony) (5551)

\#5 (lumbal or lumbar* or lumbosacral or lumbo-sacral or lumbosacroiliac or lumbo sacroiliac or spine or spinal) near/3 (pain or pains or painful* or pained or hurt or hurts or hurting or sore or soreness or tender* or discomfort or aching or agony or ache* or backache* or backpain*) (2594) 
\#6 (lumbar* or lumbal or lumbosacral or lumbo-sacral) near/2 syndrome* (105)

\#7 lumbago or lumbalgesia or lumbalgia or lumbodynia (239)

\#8 \#1 or \#2 or \#3 or \#4 or \#5 or \#6 or \#7 (7256)

\#9 [mh "Behavior Therapy"] (11029)

\#10 [mh "Conditioning (Psychology)"] (604)

\#11 (behavio* ${ }^{*}$ or cognitive or implosive or implosion or aversion) near/3 (therap* or treatment* or training or modify or modification or modified) (23667)

\#12 desensiti*ation or CBT (5476)

\#13 conditioning or (conditional next stimul*) or (conditioned next behavio* ${ }^{*}$ ) or (operant next treatment*) or (operant next behavio** $\left.{ }^{*}\right)(4495)$

\#14 [mh "Patient Education as Topic"] (6777)

\#15 (patient* or consumer* or health) near/3 (educat* or learn* or teach* or train*) (24585)

\#16 psychoeducat* ${ }^{*}$ (1502)

\#17 graded near/3 (activit* or exposure*) (230)

\#18 [mh "self Care"] (4072)

\#19 "self care" or (self next regulat*) or (self next manag*) or (self next monitor*) or "self help" (10702)

\#20 [mh "Physical Therapy Modalities"] (16607)

\#21 [mh "Physical Therapists"] (35)

\#22 physiotherap* or (physio next therap*) or (physical near/2 therap*) (15681)

\#23 (psychosocial or "psycho social" or "bio psychosocial" or "bio psycho social" or "biopsycho social") near/3 (care* or intervention* or therap* or treatment* or support* or rehab*) (2971)

\#24 \#9 or \#10 or \#11 or \#12 or \#13 or \#14 or \#15 or \#16 or \#17 or \#18 or \#19 or \#20 or \#21 or \#22 or \#23 (83959)

\#25 [mh "primary Health Care"] (4040)

\#26 "primary care" or "primary health care" or (primary next health next service*) or "primary healthcare" or "primary medical care" (15313)

\#27 \#25 or \#26 (16020)

\#28 \#8 and \#24 and \#27 (305)

$\begin{array}{ll}\text { CDSR } & 89 \\ \text { CENTRAL } & 171 \\ \text { DARE } & 6 \\ \text { HTA } & 5\end{array}$

PEDro (http://www.pedro.org.au/): up to 3 November 2015

Date searched: 1.12.15 


\section{Advanced Search}

\begin{tabular}{llc}
\hline Search Fields & Search Terms & Results \\
\hline Abstract \& Title & biopsychosocial & 21 \\
AND & lumbar spine, sacro-iliac joint or pelvis & \\
Body Part* & psychosocial & 69 \\
Abstract \&Title & lumbar spine, sacro-iliac joint or pelvis & \\
AND & & 90 \\
Body Part* & & 86 \\
Total & & \\
Total after removal of duplicates & &
\end{tabular}

*Predetermined list of terms 
APPENDIX II ADAPTED VERSION OF THE NATIONAL INSTITUTES OF HEALTH BEHAVIOR CHANGE CONSORTIUM (NIHBCC) CHECKLIST

\begin{tabular}{|c|c|c|c|}
\hline \multicolumn{4}{|l|}{ Treatment design } \\
\hline \multirow[t]{3}{*}{ Type, number \& length (min) of contacts } & Type & Number & Length (min) \\
\hline & Individual & & \\
\hline & Group & & \\
\hline \multicolumn{4}{|l|}{ Number of participants per group } \\
\hline \multicolumn{4}{|l|}{ Number of therapists per group } \\
\hline \multicolumn{4}{|l|}{ Frequency of contacts } \\
\hline \multicolumn{4}{|l|}{ Duration of contact over time } \\
\hline \multicolumn{4}{|l|}{$\begin{array}{l}\text { Theoretical model or clinical guidelines } \\
\text { on which the intervention is based }\end{array}$} \\
\hline \multicolumn{4}{|l|}{ Specified active ingredients of the intervention } \\
\hline \multicolumn{4}{|l|}{ Additional support for therapist/patients } \\
\hline \multicolumn{4}{|l|}{ Characteristics of therapists } \\
\hline \multicolumn{4}{|l|}{ Number of therapists } \\
\hline \multicolumn{4}{|l|}{ Expertise of therapists } \\
\hline \multicolumn{4}{|l|}{ Years/level of experience } \\
\hline \multirow{2}{*}{\multicolumn{4}{|c|}{$\begin{array}{l}\text { Training programme therapists } \\
\text { Construction of training programme therapists }\end{array}$}} \\
\hline & & & \\
\hline \multirow[t]{3}{*}{ Type, number \& length of contact } & Type & Number & Length (min) \\
\hline & Individual & & \\
\hline & Group & & \\
\hline \multicolumn{4}{|l|}{ Intensity of contacts } \\
\hline \multicolumn{4}{|l|}{ Duration of contact over time } \\
\hline \multicolumn{4}{|l|}{ Differences in dose between therapists } \\
\hline \multicolumn{4}{|l|}{ Additional supervision } \\
\hline \multicolumn{4}{|l|}{ Duration of additional supervision over time } \\
\hline \multicolumn{4}{|l|}{$\begin{array}{l}\text { Theoretical model, clinical guidelines or previous studies } \\
\text { on which the training programme is based } \\
\end{array}$} \\
\hline \multicolumn{4}{|l|}{ Specified active ingredients of the training programme } \\
\hline \multicolumn{4}{|l|}{ Orientation of therapists (biomedical, biopsychosocial) } \\
\hline \multicolumn{4}{|l|}{ Delivery of Treatment } \\
\hline \multicolumn{4}{|l|}{ Differences in dose between participants (e.g. attendance list) } \\
\hline \multicolumn{4}{|l|}{$\begin{array}{l}\text { Adherence of therapists to intervention plan (audiotape, observation, } \\
\text { self-report of provider, exit interview with participant) }\end{array}$} \\
\hline \multicolumn{4}{|l|}{ Receipt of treatment } \\
\hline \multicolumn{4}{|l|}{$\begin{array}{l}\text { Did the participant comprehend the intervention } \\
\text { during the intervention period? }\end{array}$} \\
\hline \multicolumn{4}{|l|}{$\begin{array}{l}\text { Did the therapist use strategies to improve participant comprehension? } \\
\text { (review homework or self-monitoring logs; ask questions/discuss } \\
\text { material; complete activity logs; conduct structured interviewed } \\
\text { with participants; work with subjects until they can demonstrate } \\
\text { the skills; monitor and give feedback on practice sessions. }\end{array}$} \\
\hline $\begin{array}{l}\text { Did the study assess the participant's ability } \\
\text { to perform the intervention skills? }\end{array}$ & & & \\
\hline $\begin{array}{l}\text { Did the study use strategies to improve participant performance } \\
\text { of intervention skills during the intervention period }\end{array}$ & & & \\
\hline
\end{tabular}




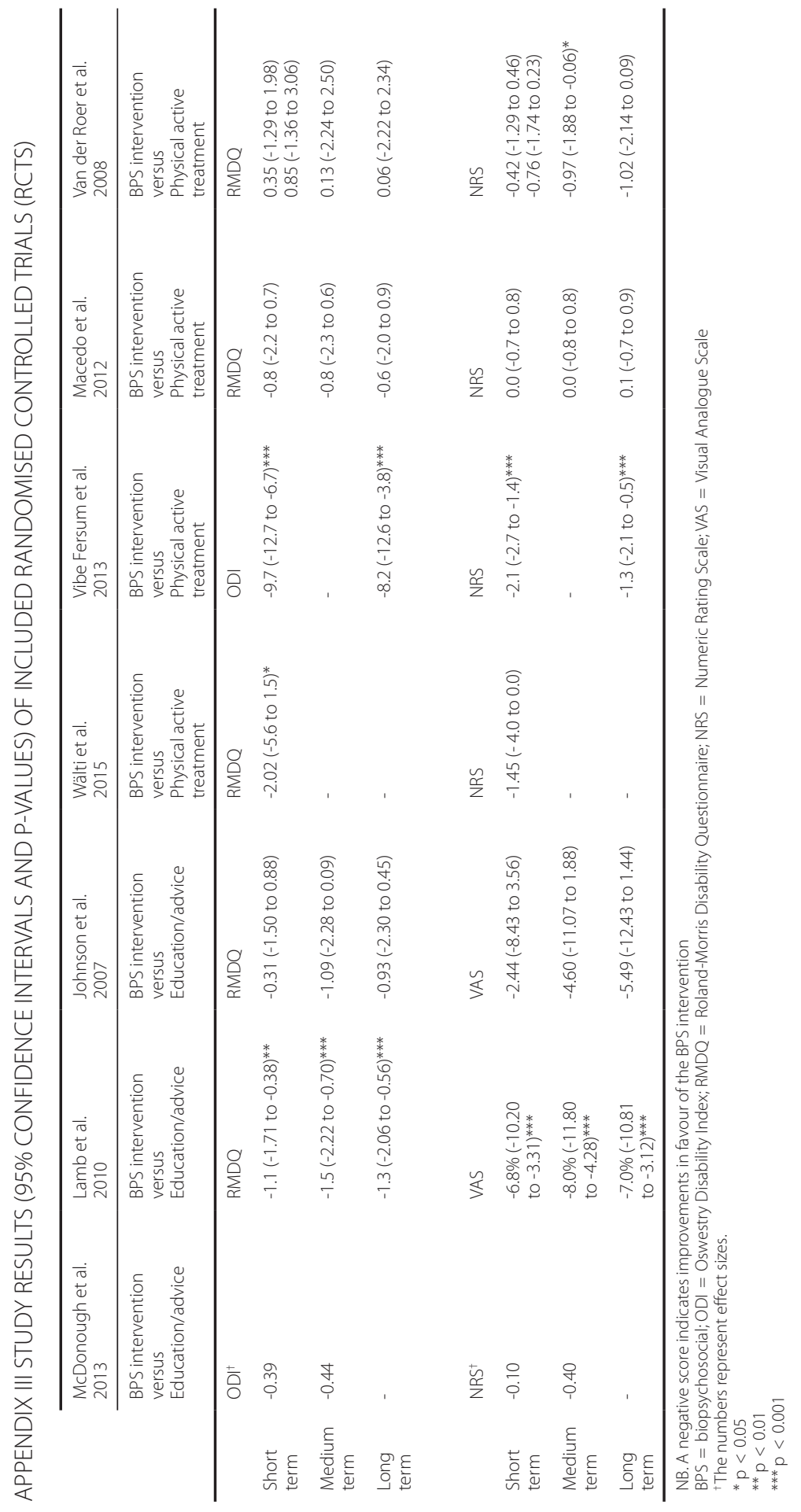




\title{
Chapter 3
}

\section{Development and content of the biopsychosocial primary care intervention 'Back on Track' for a subgroup of people with chronic low back pain}

\author{
Reni van Erp \\ Ivan Huijnen \\ Albère Köke \\ Frans Abbink \\ Marlies den Hollander \\ Rob Smeets
}





\section{ABSTRACT}

Biopsychosocial interventions provided in multidisciplinary settings are promising for improving functional disability levels in patients with chronic low back pain (CLBP). These multidisciplinary biopsychosocial interventions mainly focus on cognitive-behavioural approaches that aim to change negative cognitions, emotions, behaviour, work and social factors. As some patients with CLBP treated in primary care settings also experience psychosocial factors that influence their level of disability, these patients may benefit from the provision of a biopsychosocial intervention in primary care. This paper will provide a detailed description of the development and content of the biopsychosocial primary care intervention 'Back on Track' for this specific subgroup of patients. The Back on Track intervention was developed based on available scientific evidence and clinical experience from multidisciplinary pain rehabilitation programmes, and its effectiveness is currently being tested.

\section{KEYWORDS}

Low back pain, chronic pain, cognitive behavioural therapy, primary health care, physical therapy 


\section{INTRODUCTION}

Developing effective interventions for patients with non-specific chronic low back pain (CLBP) is challenging. Despite the fact CLBP is very common, affecting approximately $21 \%$ of the general adult population (> 18 years), ${ }^{1}$ there is still ongoing debate about the most appropriate treatment strategy. As no medical cure for pain exists, many treatments, based on a biopsychosocial model, focus on teaching patients how to cope with their pain and pain-related disability. Examples are exercise therapy, back schools, psychological or behavioural treatment, and multi-disciplinary rehabilitation. ${ }^{2}$ At present, national and inter-national guidelines recommend (multidisciplinary) biopsychosocial interventions for the management of CLBP. ${ }^{3-5}$ Also, systematic reviews analysing randomised controlled trials (RCTs) found more promising results for multidisciplinary biopsychosocial interventions than other treatments or waiting lists for the improvement of disability levels., ${ }^{2,6}$

Biopsychosocial interventions combine different treatment modalities to target physical, psychological and social factors based on the assumption that (a combination of) psychological and social factors are related to the persistence of chronic pain and the associated level of disability. ${ }^{78}$ Well-known psychological factors are negative or catastrophizing thoughts that some patients develop after injury or due to the experience of persistent pain. For example, patients think or expect that certain activities or movements will cause (re)injury. Due to these negative thoughts, patients develop pain-related fear (i.e. being afraid to harm themselves or experience pain when performing functional activities). This leads to fear-avoidance behaviour, resulting in higher levels of inactivity and functional disability. Other patients may not avoid activities completely but act 'pain contingently', and stop performing activities once they experience pain. Overtime, this can lead to higher functional disability?

However, CLBP is not limited solely to the patient, but also affects the social environment. For example, a spouse might need to take more responsibility for household or parenting activities, and relationships with colleagues and friends might change due to reduced ability to perform social or work-related activities. Overall, it is suggested that patients with less social support at home/work are more likely to develop CLBP. ${ }^{9}$ However, where family members, friends or colleagues are supportive but have negative perceptions about (the consequences of) pain themselves, they might advise patients to avoid activities causing pain, to take medicine or to rest in bed. ${ }^{10,11}$ They can unconsciously restrict a patient's performance of daily activities and reinforce passive coping styles, and by doing so, play a significant role in the patient's pain problem.

The types of psychosocial factors present and the degree to which they influence CLBP varies between patients. Heterogeneity in profile subsequently leads to heterogenic responses to interventions. ${ }^{12}$ Therefore, identification of psychosocial factors by healthcare professionals seems to be of fundamental importance to provide the most suitable treatment. ${ }^{13,14}$

In current daily practice, patients with complex (severe) psychosocial factors reporting high levels of functional disability are the main recipients of biopsychosocial interventions in multidisciplinary pain rehabilitation settings. Patients with less complex or no psychosocial factors receive interventions in primary care settings. These interventions generally include information or advice (32\%), exercise therapy (81\%) and manual therapy (massage, manual manipulation; 76\%). ${ }^{15}$ 
Based on the assumption that primary care patients might also benefit from biopsychosocial interventions, a new biopsychosocial primary care intervention ('Back on Track') has been developed. This intervention aims specifically at identification and modification of influencing psychosocial factors. The Back on Track intervention is currently under evaluation and will be compared with primary care physiotherapy in a multicentre $(n=8)$ double-blind RCT. ${ }^{16}$ Usually, publications about clinical trials provide limited information about the content of the intervention. This article has therefore been compiled to give a detailed description of the biopsychosocial Back on Track intervention to facilitate healthcare providers in understanding and reproducing biopsychosocial interventions in clinical practice.

\section{DEVELOPMENT OF THE BACK ON TRACK INTERVENTION}

The Back on Track intervention is a primary care physiotherapy treatment developed by Dutch physiatrists, psychologists and physiotherapists working in primary and secondary healthcare settings. Furthermore, members of the Dutch Association of Back Pain 'de Wervelkolom' were invited to provide feedback. The Back on Track intervention is based on available scientific evidence and currently applied evidence-based multidisciplinary pain rehabilitation programmes used at the Department of Rehabilitation Medicine, Maastricht UMC+ (Maastricht, The Netherlands) and Adelante, Centre of Expertise in Rehabilitation and Audiology (Hoensbroek, The Netherlands). Multidisciplinary pain rehabilitation programmes aim to improve daily activity levels by using cognitive-behavioural approaches such as graded activity (GA) and graded exposure in vivo (EXP) in combination with pain education. GA and EXP have been shown to be equally effective for improving functional disability inpatients with CLBP. ${ }^{17,18}$ Pain education as an integral part of GA and EXP or on its own has also been shown to be effective. ${ }^{19}$ The Back on Track intervention includes the abovementioned approaches but at a lower intensity, as patients in primary care often experience less severe psychosocial factors than patients referred to multidisciplinary pain rehabilitation programmes. In general, the main objectives of the Back on Track intervention are:

- To understand pain mechanisms and differences between acute and chronic pain;

- To become aware of the role of physical, cognitive and social factors;

- To become aware of the influence of pain-behaviour on short and long term;

- To reduce negative/catastrophizing thoughts, pain-related fear and fear-avoidance behaviours/compensatory behaviour;

- To stimulate active coping styles by providing different self-management strategies;

- To reduce functional disability levels. 


\section{THE BACK ON TRACK INTERVENTION FORMAT}

\section{INTENSITY AND DURATION}

The Back on Track intervention consists of four individual and eight group sessions (Table 1). Within this format, 8 hours will be used for education/discussion and 2 hours will be used for performing physical activity. From the literature, it is known that education of at least 2.5 hours is more effective than less intensive or no education. ${ }^{20}$ In exercise therapy, no differences in effectiveness on disability levels are reported between extensive (20 to 30 sessions of 30 minutes) and shorter (six to 10 sessions of 30 minutes) interventions. ${ }^{21}$ As no standards are available about the intensity of interventions, the dose/intensity of the Back on Track intervention is based on the available resources in primary care, competence of physiotherapists, and an amount that will presumably induce behavioural changes.

Table 1 Schematic view of the Back on Track intervention

\begin{tabular}{llc}
\hline Sessions & Content/strategy & Duration (minutes) \\
\hline Individual & History taking & 30 \\
& Pain-education & 30 \\
& Goal setting & 30 \\
Theme 1-Pain \& physical activity & 60 \\
Session 1 education & $30+30$ \\
& Session 2 education + physical activity & 60 \\
Theme 2-Pain \& social network & $30+30$ \\
& Session 1 education & 60 \\
& Session 2 education + physical activity & $30+30$ \\
& Theme 3-Pain \& cognitions & \\
Session 1 education & 60 \\
Session 2 education + physical activity & $30+30$ \\
Theme 4-Fact or myth? & 30 \\
Session 1 education & Session 2 education + physical activity & \\
Individual & Evaluation &
\end{tabular}

The total duration of the programme is approximately 8 weeks when provided twice per week. As more than half of primary care patients with CLBP receive at least 10 physiotherapy sessions over a treatment period of at least 6 weeks in daily practice, ${ }^{22}$ it is expected that the intervention would be feasible to implement. 


\section{INDIVIDUAL AND GROUP SESSIONS}

The Back on Track intervention includes both individual and group sessions. Individual sessions are included to gain detailed insight in the patient-specific situation (history, psychosocial factors, goals) and to enhance the patient-therapist relationship. Group sessions are included to stimulate discussion between patients (e.g. about influencing psychosocial factors, difficulties in life and differences in pain behaviour or coping styles). Discussing these topics can lead to mutual support and observational learning which may stimulate active coping styles (i.e. observing positive consequences of certain behaviour in other patients may inspire confidence and may lead to imitation of these coping styles). ${ }^{23,24}$ Another advantage of group therapy is the potential cost-effectiveness compared with individual therapy. ${ }^{25}$ Back on Track groups include three to five patients, and are administered by one physiotherapist. A minimum of three patients is chosen as smaller group sizes are expected to lead to less group interaction, while a maximum of five patients is chosen for practicability reasons (facilities).

\section{METHOD OF DELIVERY}

\section{WHO DELIVERS THE BACK ON TRACK INTERVENTION?}

The Back on Track intervention is designed for primary care physiotherapists. However, it has been shown that the attitude (biomedical or biopsychosocial) of a healthcare provider influences the strategy of the actual treatment and the advice provided to patients. ${ }^{26}$ Motivation and skills of the physiotherapist are therefore important prerequisites for sufficient treatment delivery.

\section{TREATMENT PROTOCOL AND EDUCATIONAL PROGRAMME}

A treatment manual with detailed theoretical and practical information has been developed to standardise practical implementation. Also, an educational programme has been developed including three 4-hour meetings to ensure basic knowledge and understanding of cognitive-behavioural approaches and accurate delivery of the intervention. The length of the educational programme was derived from the 2-day programme of the study of Lamb et al., which resulted in a well-implemented and effective primary care cognitive-behavioural-based programme. ${ }^{27,28}$

\section{INFORMATION BOOKLETS}

It has been shown that providing general information about pain and the treatment rationale are effective elements of treatment. 29,30 $^{2}$ Moreover, written information in conjunction with verbal information seems to be effective in changing pain perceptions and the health status of patients. ${ }^{30}$ As such, an information booklet has been created for patients with basic information, helpful illustrations and home assignments. 


\section{DETAILED DESCRIPTION OF THE BACK ON TRACK INTERVENTION}

\section{REFERRAL PROCEDURE}

It is favourable if a patient is referred by a healthcare professional specialised in chronic pain. Skilled professionals can identify psychosocial factors and can introduce the biopsychosocial programme by providing education, including an explanation about the current health status, the meaning of chronic pain (hurt does not mean harm), and advice to perform normal daily activities despite pain. By providing education, the health care professional can reinforce the credibility of the biopsychosocial approach, and prepare patients for a different approach than normally expected in primary care.

\section{INDIVIDUAL SESSIONS}

\section{Individual session 1}

The intervention starts with a combination of history taking and pain education using the pain-consequence model; this model is used frequently in Dutch clinical practice (Figure 1). The model consists of two parts; a biomedical part (or causal explanation of pain) and a biopsychosocial part (or consequences that contribute to chronicity). During the first session, the biomedical part is discussed to provide insight into results and successes of previously received medical diagnoses, advice and treatments. The patient must eventually start to recognise that continuing a biomedical approach is probably unproductive and, due to the complexity of the pain problem, a transition to a more biopsychosocial orientation is needed. Pain education prepares the patient for the different treatment approach (improving daily functioning despite pain and no pain reduction), and increases readiness to change coping styles. A short functional examination can also be performed to gain insight into pain behaviour during lifting, bending or dressing (e.g. avoidance or compensatory).

\section{Individual session 2}

During the second session, the attention shifts to the biopsychosocial part of the pain-consequence model. First, pain mechanisms and the transition from acute to chronic pain are discussed in order to improve knowledge and understanding about pain. Secondly, and as insight progresses, factors that a patient recognises as contributing to his/her own pain problem are discussed. At home, the patient selects the three functional activities that are most restricted due to CLBP using the Dutch Patient Specific Functional Scale (PSFS). ${ }^{31}$ 


\section{Individual session 3}

For the three most restricted and valuable functional activities selected from the PSFS, patient-specific goals are set collaboratively according to the SMART principles (i.e. specific, measurable, achievable and realistic in a certain timeframe). Homework assignment includes performing selected activities on a pain-contingent basis to set up a baseline performance measure. This baseline will eventually be used to set up a starting point for GA (Theme 1).

Figure 1 The pain-consequence model. This model acts as a framework for the Back on Track intervention. The model addresses multiple relevant biomedical factors (left) and biopsychosocial factors (right) that might contribute to the development and/or persistence of CLBP.

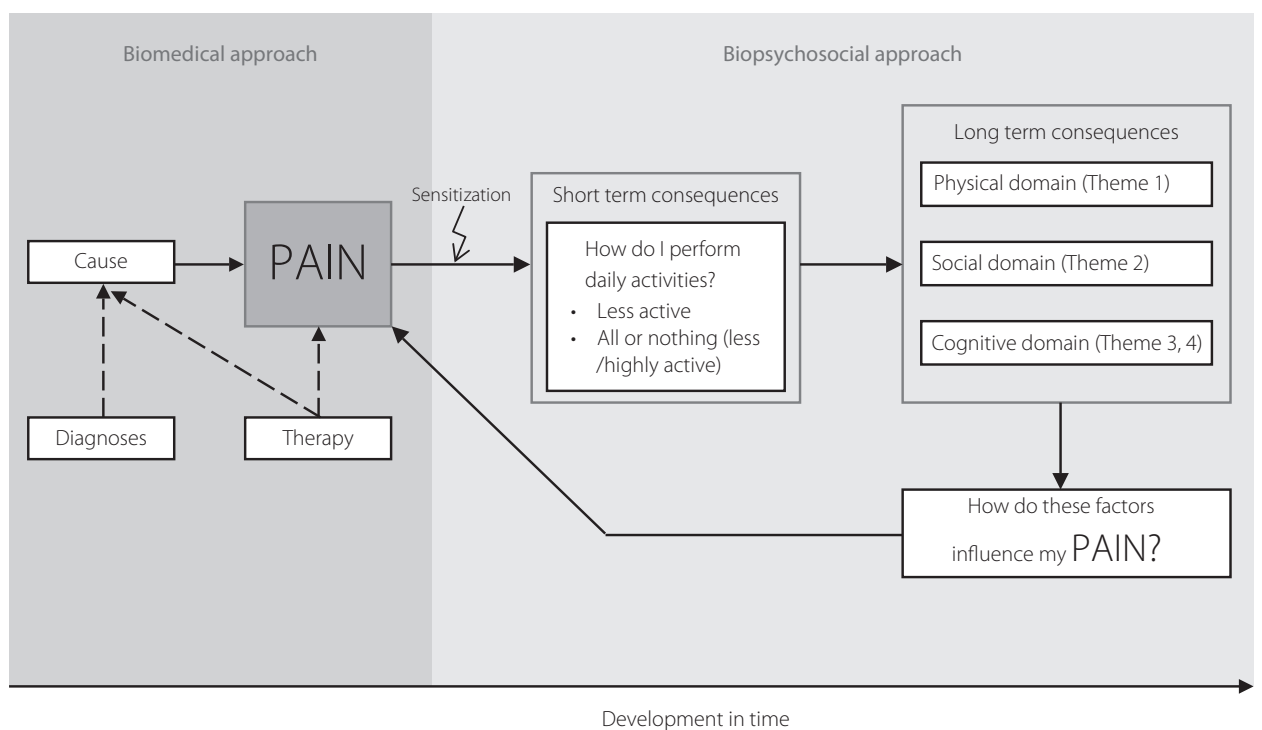




\section{GROUP SESSIONS}

After three individual sessions, eight group sessions are provided twice a week. Each week focuses on one of the four themes (Table 1). The first session of a week/theme includes 1 hour of education, while the second session includes a combination of education and physical activity. The following section provides information about each education session for each theme. Subsequently, all physical activity sessions are discussed together because of the overlap between these sessions.

\section{Theme 1. Pain \& physical activity}

Pain can stimulate a patient to stop performing activities (acting pain contingently), which often leads to pain reduction in the short term. However, in the long term, this pain behaviour can result in reduced daily life functioning. Therefore, Theme 1 discusses patients' own pain behaviour and related short and long term consequences. The operant conditioning behavioural approach, $\mathrm{GA}^{32}$ is introduced to encourage active coping styles. Furthermore, patients develop a GA plan based on self-measured activity levels (derived from homework assignment in the third individual session) in order to increase their performance levels gradually and time contingently. Patients are encouraged to use their GA plans during therapy (physical activity therapy sessions) and at home (homework assignment).

\section{Theme 2. Pain \& social network}

Social factors, such as family (spousal/partner support), work (colleague support, job stress) and health care (physiotherapist's empathy, communication), can play an important role in the development and/or persistence of chronic pain. ${ }^{9,33}$ Social factors should be recognised as external factors that can influence a patient's attitude towards pain either positively or negatively. Theme 2 therefore discusses the patient's own coping strategies in relation to coping strategies of their significant others. This might eventually stimulate lifestyle changes and pain behaviour. Home-work assignment is to write down how significant others respond whenever the patient experiences pain, and how the patient subsequently reacts to that response. In this way, a patient can learn operant-conditioning mechanisms that contribute to the maintenance of pain and pain-related disability.

\section{Theme 3. Pain \& cognitions}

As mentioned previously, some patients generate catastrophizing thoughts after the experience of pain and develop pain-related fear and kinesiophobia. This can lead to lower levels of activity and higher functional disability. I t has been shown that therapy can modify catastrophizing thinking, and a reduction in catastrophizing thinking is related to better treatment outcomes. ${ }^{34,35}$ Although the target group of the Back on Track intervention may not encounter extreme catastrophizing thoughts, a patient may still encounter negative thoughts that can lead to different 
movement strategies. ${ }^{36}$ Theme 3 therefore discusses what types of cognitions are present about daily activities. The Photograph Series of Daily Activities - Short Electronic Version is used to measure the threat value of daily activities [ranging from 0 ('not harmful at all') to 100 ('extremely harmful')] and to determine a hierarchy of the patient's fearful activities. ${ }^{37}$ At home, patients define and rate (new) threatening functional activities (homework assignment).

\section{Theme 4. Fact or myth?}

It has been shown that misbeliefs about low back pain still exist in the general population (e.g. the need for bedrest, or the need to use radiographs or other imaging tests to identify the cause of pain). ${ }^{38}$ Misbeliefs may be caused due to the inability of professionals to translate information to a patient's level, poor non-scientific sources of information available in the media, or the inability of the patient to interpret information appropriately. Theme 4 therefore aims to discuss (and to reach consensus) about facts and myths with regard to low back pain. Discussions about misbeliefs will improve knowledge and may indirectly stimulate active coping styles. In case uncertainties or questions are still present after this session, they must be recorded by the patient (homework) and discussed in subsequent sessions.

This session also addresses the impact of helpful (positive) or non-helpful (negative) thoughts on mood and disability level. It is important to clarify what type of thoughts, either positively or negatively tuned, the patient experiences in certain situations such as waking up, going to a birthday party, sports, work etc. At home, the patient should write down their own thoughts and feelings in different situations, and determine whether these cognitions are positively or negatively tuned.

\section{Physical active sessions}

The four physical activity sessions are provided as a second session each week and start by repeating cognitive-behavioural principles, previous discussions and self-management strategies. Secondly, sports activities are performed. The main aim of these sessions is to become physically active, to inspire confidence and to have pleasure in being physically active. Physiotherapists observe behaviour and confront the patient with their own (unconscious) pain behaviour. Sports activities are based on the preferences of the group and the available sports facilities. The type or content of sports activities is therefore not protocolled, apart from Theme 3.

During the physical activity session of Theme 3, the patient is challenged to test their own negative cognitions using EXP principles. EXP exposes patients to fearful functional activities in order to experience the inconsistency of previous expectations with actual consequences of the activity. Eventually, this leads to reduced pain-related fear and improved functional activity levels..$^{39,40}$

Homework assignment for physical activity sessions is to improve functional activities by executing personal GA plans or immediate exposure. 


\section{EVALUATION SESSION}

At the end of the intervention, an individual evaluation session is provided to assess the experiences of the patient, the actual status, improvements in functional disability levels and ability to generalise perceived knowledge into home situations. The physiotherapist discusses difficulties and ways to cope with situations in case back pain recurs. If the improvements, generalisability and coping behaviour of the patient are not sufficient, referral back to the referring healthcare professional is indicated.

\section{DISCUSSION}

This article provides a description of the biopsychosocial primary care intervention 'Back on Track'. This intervention is specifically developed for patients with CLBP who experience non-complex psychosocial factors and in whom the level of disability is low to moderate. The intervention is based on available scientific evidence and clinical experience from multidisciplinary pain rehabilitation programmes. Currently, the intervention is under evaluation in an RCT, the results of which are expected to be available in June 2017.The detailed description of the RCT design can be found elsewhere. ${ }^{16}$ If the intervention proves to be effective, this article may facilitate other healthcare professionals to implement biopsychosocial interventions in clinical practice.

\section{ACKNOWLEDGEMENTS}

The authors wish to thank Jeanine Verbunt, Paul Willems and the Dutch Association of Back Patients 'de Wervelkolom' for their contribution to the development of the Back on Track intervention. Furthermore, the authors wish to thank the Department of Rehabilitation in Medicine MUMC +, Spine Centre MUMC + and Fy'net Collaboration for management of the trial. 


\section{REFERENCES}

1. Picavet HS, Schouten JS. Musculoskeletal pain in the Netherlands: prevalences, consequences and risk groups, the DMC(3)-study. Pain. 2003;102(1-2):167-178.

2. van Middelkoop M, Rubinstein SM, Kuijpers T, et al. A systematic review on the effectiveness of physical and rehabilitation interventions for chronic non-specific low back pain. European spine journal : official publication of the European Spine Society, the European Spinal Deformity Society, and the European Section of the Cervical Spine Research Society. 2011;20(1):19-39.

3. Royal Dutch Society for Physical Therapy. (KNGF). KNGF evidence-based clinical practice guidelines. 2013; https://www.fysionet-evidencebased.nl/index.php/kngf-guidelines-in-english Accessed Febr 12, 2016.

4. Airaksinen O, Brox Jl, Cedraschi C, et al. Chapter 4. European guidelines for the management of chronic nonspecific low back pain. European spine journal : official publication of the European Spine Society, the European Spinal Deformity Society, and the European Section of the Cervical Spine Research Society. 2006;15 Suppl 2:S192-300.

5. Dutch Institute for Healthcare Improvement. (CBO). Clinical guideline for non-specific low back pain [in Dutch]. Alphen aan den Rijn2003.

6. Kamper SJ, Apeldoorn AT, Chiarotto A, et al. Multidisciplinary biopsychosocial rehabilitation for chronic low back pain. The Cochrane database of systematic reviews. 2014;9:CD000963.

7. Vlaeyen JW, Linton SJ. Fear-avoidance and its consequences in chronic musculoskeletal pain: a state of the art. Pain. 2000;85(3):317-332.

8. Waddell G. The back pain revolution. 2nd ed. ed. Oxford: Churchill Livingstone; 2004.

9. Melloh M, Salathe CR, Elfering A, et al. Occupational, personal and psychosocial resources for preventing persistent low back pain. International journal of occupational safety and ergonomics : JOSE. 2013;19(1):29-40.

10. Fordyce WE. Behavioral Methods for Chronic Pain and IIIness. St. Louis, MO: Mosby; 1976.

11. Leonard MT, Cano A, Johansen AB. Chronic pain in a couples context: a review and integration of theoretical models and empirical evidence. The journal of pain : official journal of the American Pain Society. 2006;7(6):377-390.

12. George SZ, Fritz JM, Bialosky JE, Donald DA. The effect of a fear-avoidance-based physical therapy intervention for patients with acute low back pain: results of a randomized clinical trial. Spine. 2003;28(23):2551-2560.

13. Hill JC, Dunn KM, Lewis M, et al. A primary care back pain screening tool: identifying patient subgroups for initial treatment. Arthritis and rheumatism. 2008;59(5):632-641.

14. Vibe Fersum K, O'Sullivan P, Skouen JS, Smith A, Kvale A. Efficacy of classification-based cognitive functional therapy in patients with non-specific chronic low back pain: a randomized controlled trial. European journal of pain. 2013;17(6):916-928.

15. Swinkels IC, van den Ende CH, van den Bosch W, Dekker J, Wimmers RH. Physiotherapy management of low back pain: does practice match the Dutch guidelines? The Australian journal of physiotherapy. 2005;51(1):35-41. 
16. Erp RM, Huijnen IP, Verbunt JA, Smeets RJ. A biopsychosocial primary care intervention (Back on Track) versus primary care as usual in a subgroup of people with chronic low back pain: protocol for a randomised, controlled trial. J Physiother. 2015.

17. Leeuw M, Goossens ME, van Breukelen GJ, et al. Exposure in vivo versus operant graded activity in chronic low back pain patients: results of a randomized controlled trial. Pain. 2008;138(1):192-207.

18. George SZ, WittmerVT, Fillingim RB, Robinson ME. Comparison of graded exercise and graded exposure clinical outcomes for patients with chronic low back pain. The Journal of orthopaedic and sports physical therapy. 2010;40(11):694-704.

19. Louw A, Diener I, Butler DS, Puentedura EJ. The effect of neuroscience education on pain, disability, anxiety, and stress in chronic musculoskeletal pain. Archives of physical medicine and rehabilitation. 2011;92(12):2041-2056.

20. Engers A, Jellema P, Wensing M, van der Windt DA, Grol R, van Tulder MW. Individual patient education for low back pain. The Cochrane database of systematic reviews. 2008(1):CD004057.

21. Ferreira ML, Smeets RJ, Kamper SJ, Ferreira PH, Machado LA. Can we explain heterogeneity among randomized clinical trials of exercise for chronic back pain? A meta-regression analysis of randomized controlled trials. Physical therapy. 2010;90(10):1383-1403.

22. Engers AJ, Schers H, Oostendorp RAB. Het handelen van Nederlandse fysiotherapeuten bij lage-rugpijn: een beschrijvend onderzoek van de dagelijkse praktijk. Ned Tijdschr Fysiother. 2001;6:142-151.

23. Carnes D, Homer KE, Miles CL, et al. Effective delivery styles and content for self-management interventions for chronic musculoskeletal pain: a systematic literature review. The Clinical journal of pain. 2012;28(4):344-354.

24. Bandura A. Social Foundations of Thought and Action: A Social Cognitive Theory. Englewood Cliffs, NJ: Prentice Hall; 1986.

25. Critchley DJ, Ratcliffe J, Noonan S, Jones RH, Hurley MV. Effectiveness and cost-effectiveness of three types of physiotherapy used to reduce chronic low back pain disability: a pragmatic randomized trial with economic evaluation. Spine. 2007;32(14):1474-1481.

26. Bishop A, Foster NE, Thomas E, Hay EM. How does the self-reported clinical management of patients with low back pain relate to the attitudes and beliefs of health care practitioners? A survey of UK general practitioners and physiotherapists. Pain. 2008;135(1-2):187-195.

27. Lamb SE, Hansen Z, Lall R, et al. Group cognitive behavioural treatment for low-back pain in primary care: a randomised controlled trial and cost-effectiveness analysis. Lancet. 2010;375(9718):916-923.

28. Lamb SE, Mistry D, Lall R, et al. Group cognitive behavioural interventions for low back pain in primary care: extended follow-up of the Back Skills Training Trial (ISRCTN54717854). Pain. 2012;153(2):494-501.

29. Del Bano-Aledo ME, Medina-Mirapeix F, Escolar-Reina P, Montilla-Herrador J, Collins SM. Relevant patient perceptions and experiences for evaluating quality of interaction with physiotherapists during outpatient rehabilitation: a qualitative study. Physiotherapy. 2014;100(1):73-79.

30. Nijs J, Paul van Wilgen C, Van Oosterwijck J, van Ittersum M, Meeus M. How to explain central sensitization to patients with 'unexplained' chronic musculoskeletal pain: practice guidelines. Manual therapy. 2011;16(5):413-418.

31. Beurskens AJ, de Vet HC, Koke AJ, et al. A patient-specific approach for measuring functional status in low back pain. J Manipulative Physiol Ther. 1999;22(3):144-148. 
32. Lindstrom I, Ohlund C, Eek C, et al. The effect of graded activity on patients with subacute low back pain: a randomized prospective clinical study with an operant-conditioning behavioral approach. Physical therapy. 1992;72(4):279-290; discussion 291-273.

33. Shaw WS, Campbell P, Nelson CC, Main CJ, Linton SJ. Effects of workplace, family and cultural influences on low back pain: what opportunities exist to address social factors in general consultations? Best practice \& research Clinical rheumatology. 2013;27(5):637-648.

34. Smeets RJ, Vlaeyen JW, Kester AD, Knottnerus JA. Reduction of pain catastrophizing mediates the outcome of both physical and cognitive-behavioral treatment in chronic low back pain. The journal of pain : official journal of the American Pain Society. 2006;7(4):261-271.

35. Wertli MM, Burgstaller JM, Weiser S, Steurer J, Kofmehl R, Held U. Influence of catastrophizing on treatment outcome in patients with nonspecific low back pain: a systematic review. Spine. 2014;39(3):263273.

36. Thomas JS, France CR. Pain-related fear is associated with avoidance of spinal motion during recovery from low back pain. Spine. 2007;32(16):E460-466.

37. Leeuw M, Goossens ME, van Breukelen GJ, Boersma K, Vlaeyen JW. Measuring perceived harmfulness of physical activities in patients with chronic low back pain: the Photograph Series of Daily Activities - short electronic version. The journal of pain : official journal of the American Pain Society. 2007:8(11):840-849.

38. Ihlebaek C, Eriksen HR. The "myths" of low back pain: status quo in norwegian general practitioners and physiotherapists. Spine. 2004;29(16):1818-1822.

39. Vlaeyen JW, de Jong J, Geilen M, Heuts PH, van Breukelen G. Graded exposure in vivo in the treatment of pain-related fear: a replicated single-case experimental design in four patients with chronic low back pain. Behaviour research and therapy. 2001;39(2):151-166.

40. Vlaeyen JW, de Jong J, Geilen M, Heuts PH, van Breukelen G. The treatment of fear of movement/(re) injury in chronic low back pain: further evidence on the effectiveness of exposure in vivo. The Clinical journal of pain. 2002;18(4):251-261. 



\title{
Chapter 4
}

\author{
A biopsychosocial primary care \\ intervention (Back on Track) versus \\ primary care as usual in a subgroup of \\ patients with chronic low back pain; \\ protocol for a randomised controlled trial
}

\author{
Reni van Erp \\ Ivan Huijnen \\ Jeanine Verbunt \\ Rob Smeets
}





\section{ABSTRACT}

Introduction: Multidisciplinary biopsychosocial interventions are effective in improving functional disability in patients experiencing chronic low back pain (CLBP). These interventions are, however, expensive and often long waiting times exist before treatment can start. Implementing biopsychosocial interventions in primary care settings might therefore be interesting. Since patients with CLBP show different biopsychosocial profiles, patients might respond differently to specific interventions.

Research questions: This study will investigate the difference in (cost-) effectiveness between a biopsychosocial primary care intervention Back on Track and primary care physiotherapy as usual in a subgroup of patients with CLBP.

Design: Double-blind, multicentre $(n=8)$ randomised controlled trial.

Participants: Eighty-six patients with CLBP, aged 18-65 yr., experiencing low to moderate levels of disability and in whom the contributing role of psychosocial factors to this disability is restricted.

Intervention: The Back on Track intervention; 4 individual and 8 group sessions, based on biopsychosocial approaches from multidisciplinary pain rehabilitation programmes, and provided by trained physiotherapists.

Control: Primary care physiotherapy as usual.

Measurements: Primary outcome is functional disability (Quebec Back Pain Disability Scale) at post-treatment, 3 and 12 months of follow-up. Secondary measures are credibility and expectancy, anxiety and depression, catastrophizing, pain intensity, kinesiophobia, self-efficacy, patient's global perceived effect, cost-effectiveness, and cost-utility estimated with cost diaries and quality-adjusted life years.

Analysis: Linear mixed models using an intention-to-treat principle. Incremental cost-effectiveness and cost-utility ratios will be calculated and plotted on a cost-effectiveness plane.

Discussion: This study will provide useful information on a biopsychosocial intervention for chronic low back pain in primary care settings. 


\section{INTRODUCTION}

Low back pain (LBP) is the most prevalent self-reported musculoskeletal pain complaint in the Netherlands. ${ }^{1}$ In the majority of patients experiencing LBP (90\%) no biomedical abnormalities can be found explaining the low-back complaints. ${ }^{2}$ These cases are called non-specific LBP. One in five patients with non-specific LBP eventually develops chronic complaints (CLBP), indicating persistent LBP for more than 3 months. ${ }^{3}$ CLBP has nowadays been recognised as a complex phenomenon in which multiple physical, psychological and social factors are assumed to be related to the development and maintenance of CLBP. Since most patients remain seeking a solution for their complex and unsolvable pain problem (medical shopping behaviour) and often show long term work absenteeism, CLBP leads to high medical as well as societal costs., ${ }^{3,4}$ Due to a growing and aging population it is expected that in future the number of patients with CLBP and the accompanying medical and societal costs will even further increase. ${ }^{5}$

Patients with CLBP can vary in the level of disability they experience and the complexity of psychosocial factors (e.g. catastrophizing thoughts and avoidance behaviours) influencing the development and/or maintenance of the level of CLBP associated disability. ${ }^{6}$ Moreover, due to this variability in patients with CLBP, patients respond differently to interventions. ${ }^{6,7}$ In the Netherlands, Dutch physiatrists therefore determine the complexity of psychosocial factors and the associated disability level in patients with CLBP and refer patients based on their biopsychosocial profile to different therapy settings (for a summary of the classification system and health care policy for patients with chronic low back pain in the Netherlands see appendix). Patients with more complex psychosocial factors and a higher level of disability are referred to specialised multidisciplinary pain rehabilitation settings, while patients with only a restricted influence of psychosocial factors and a lower level of disability are referred to either general multidisciplinary settings not specialised in pain rehabilitation or primary care physiotherapy settings.

In patients with CLBP, multidisciplinary interventions with a biopsychosocial approach have proven to be effective in reducing functional disability ${ }^{8,9}$ and pain intensity. ${ }^{8-10}$ These biopsychosocial interventions specifically address psychosocial factors that are associated with the persistence of LBP-associated disability. Since such interventions address multiple factors, therapy guidelines highly recommend multidisciplinary biopsychosocial interventions in the management of LBP.,11 Multidisciplinary biopsychosocial interventions are, however, rather expensive and due to an increasing number of patients suffering from CLBP, often substantial waiting lists for treatment exist. Implementing biopsychosocial interventions in primary care settings would therefore be of main importance since primary care interventions are more accessible and less expensive. However, current primary care physiotherapists often focus on the treatment of functional impairments and mainly use exercise therapy and manual interventions (e.g. manipulations and massage) in the management of CLBP. ${ }^{12} \mathrm{~A}$ shift towards a more biopsychosocial-orientated approach in primary care might therefore be of additional value but challenging.

Some studies have already evaluated biopsychosocial interventions in primary care settings. For example, Lamb et al. showed positive effects at 12 months in functional disability and pain after a primary care cognitive-behavioural group intervention (1 individual session plus 6 
group sessions, 1.5 hours each) as compared to advice only in patients with at least moderately troublesome sub-acute or chronic LBP (> 6 weeks). ${ }^{13}$ The positive effects remained significant even after a mean follow-up time of 34 months $^{14}$ and remained cost-effective after one year as well. ${ }^{13}$ In contrast, van der Roer et al. compared an intensive group training including exercise therapy plus graded activity principles (10 individual and 20 group sessions) to usual care according to the Dutch guideline for LBP and showed no significant differences in outcome regarding functional status, pain intensity, coping strategies and self-efficacy. ${ }^{15}$ The inconsistency in findings between Lamb et al. and van der Roer et al. might be related to the contrast between the experimental and control intervention (i.e. using advice as control intervention (Lamb et al.) or an active intervention without a behavioural focus (van der Roer et al.)), but might also be related to the differences in the content of the experimental intervention that was tested. Where Lamb et al. focused on coping styles, fear-avoidance based principles and catastrophizing thoughts, van der Roer et al. mainly focused on operant conditioning behavioural principles, back school and exercise.

It is suggested that patients with CLBP respond differently to interventions due to the fact patients vary in the level of disability and the complexity of disabling psychosocial factors. For example, patients with a higher degree of fear-avoidance behaviour seem to benefit more from fear-avoidance targeted therapies than patients with no or a low degree of fear-avoidance behaviour. ${ }^{\text {So }}$ in order to investigate which primary care treatment is best suitable for patients with CLBP, it might be of considerable importance to execute appropriate selection by professionals prior to the start of the treatment, to refer homogeneous groups of patients towards appropriate therapy settings and to provide therapy within this therapy setting which is adjusted to the needs of the referred homogeneous group of patients. It should be noted that in the study of van der Roer et al. no specific selection criteria with regard to psychosocial factors were used, whereas Lamb et al. used a self-reported questionnaire in which patients had to self-rate the troublesomeness of their pain. In both studies, no professionals with expertise in distinguishing different psychosocial profiles were involved in the selection procedure.

Altogether, it can be presumed that biopsychosocial interventions in multidisciplinary settings are promising in patients with CLBP but that unequivocal evidence about the effectiveness of such biopsychosocial intervention in primary care is still lacking. More research is needed to investigate whether this biopsychosocial intervention in primary care is more effective compared to the regular primary care physiotherapy treatment. Patients referred to primary care physiotherapy practices experience a moderate level of disability and psychosocial factors of which the contributing role to their disability of is at maximum low. The purpose of this study is therefore to develop a biopsychosocial primary care intervention 'Back on Track' for this specific group of patients and to investigate whether this subgroup of patients will benefit more from the biopsychosocial Back on Track intervention as compared to primary care physiotherapy as usual. A cost-effectiveness evaluation as well as a process evaluation will be performed in addition. 
As mentioned before, this study will focus on patients with CLBP who experience low to moderate levels of disability and non-complex but present psychosocial factors. The specific research questions for this subgroup of patients are:

1. What is the difference in long term treatment effect (change in functional disability between pre-treatment and 12 months post-treatment) between the new primary care intervention Back on Track and primary care physiotherapy as usual?

2. What is the difference in treatment effect at 3 months post-treatment (change in functional disability between pre-treatment and 3 months post-treatment) between the new primary care intervention Back on Track and usual primary care physiotherapy?

3. What is the difference in short term treatment effect (change in functional disability between pre- and post-treatment) between the new primary care intervention Back on Track and usual primary care physiotherapy?

4. What is the difference in cost-effectiveness and cost-utility over a one-year period between the new primary care intervention Back on Track and primary care physiotherapy as usual?

\section{METHOD}

\section{DESIGN}

The study will use a pragmatic double-blind multicentre $(n=8)$ randomised controlled trial $(R C T)$. Both an effect evaluation as well as an economic evaluation will be performed. Furthermore, a process evaluation will be performed in order to evaluate treatment fidelity. Ethical approval for the Back on Track intervention study was provided by the institutional medical ethics committee of the University Hospital of Maastricht and Maastricht University (METC azM/UM; METC143019).

\section{PARTICIPANTS}

Patient recruitment will take place at the department of rehabilitation medicine of Maastricht University Medical Centre + (MUMC + ), the Netherlands, from August 2014 to August 2015. Physiatrist to whom patients are referred for treatment advice will determine the influence of psychosocial factors and the level of disability based on their clinical expertise and a set of questionnaires (pain Numeric Rating Scale (NRS), Hospital Anxiety and Depression Scale (HADS), Pain Catastrophizing Scale (PCS) and Short-Form health survey questionnaire (SF-36, physical functioning subscale). Patients complete these questionnaires prior to their visit at the physiatrist. Scores are provided to physiatrists to facilitate their clinical decision making. Patients with low to moderate levels of disability and non-complex but present psychosocial factors will be eligible for the current study. 
Figure 1 Flow diagram of the study

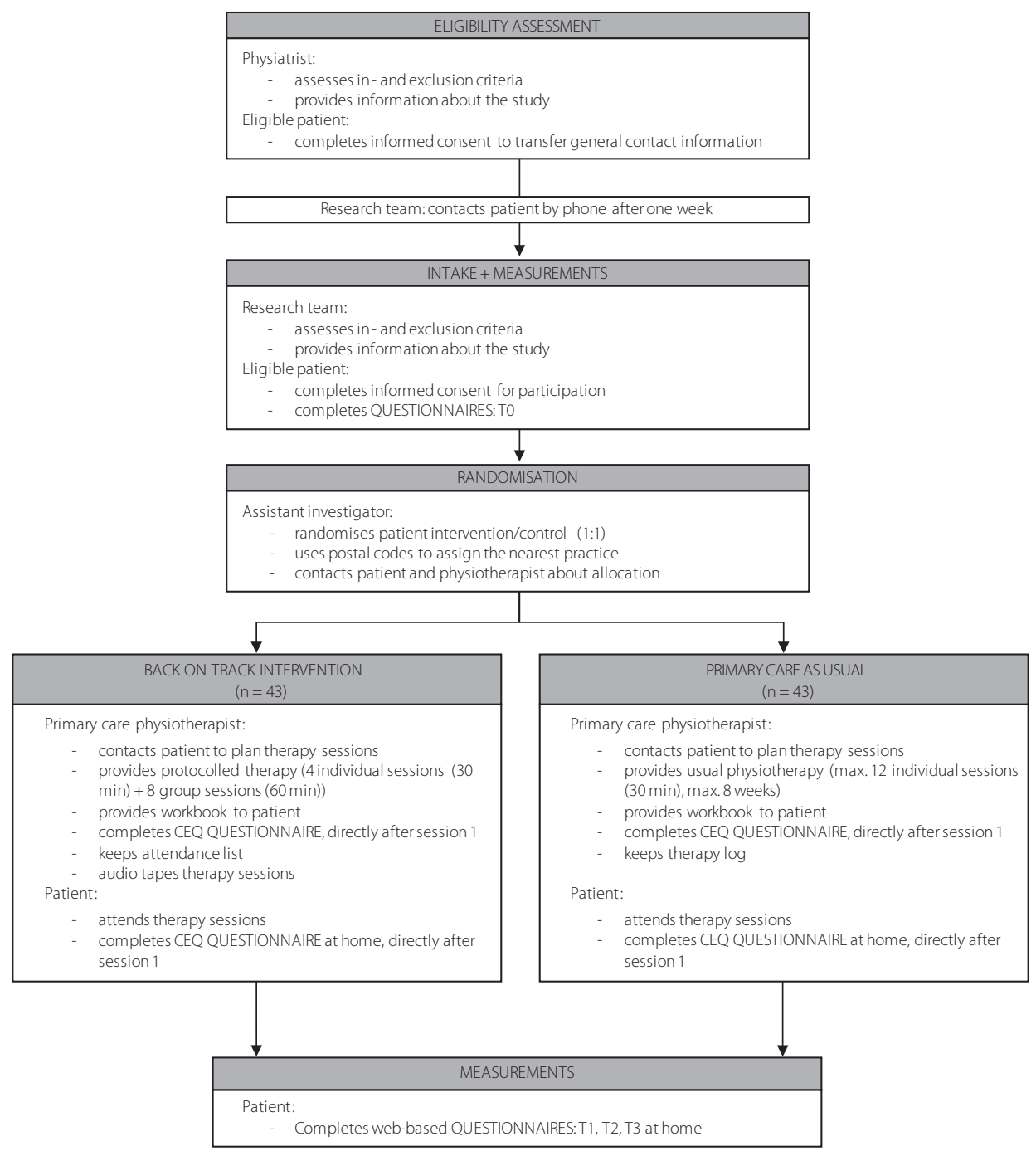


Eighty-six patients will be recruited for this study in the course of one year. In order to be eligible for participation, patients must meet all of the following inclusion criteria:

- CLBP; defined as pain between scapulae and gluteal region, whether or not with radiation towards one or both legs, present for at least three months

- Presence of contributing social and psychological factors, however not complex

- Age between 18 and 65 years

- Sufficient knowledge of the Dutch language

- Acceptance towards a biopsychosocial approach instead of a biomedical approach

Exclusion criteria are:

- CLBP pain attributable to e.g. infection, tumour, osteoporosis, fracture, structural deformity, inflammatory process, radicular syndrome or cauda equina syndrome

- Pregnancy

- Any suspicion of an (underlying) psychiatric disease, for which psychiatric treatment is better suited, according to the expert opinion of the physiatrist

In regular care, all patients receive medical education from their physiatrist irrespective of participation in the study or the allocated therapy in case of participation (Back on Track intervention or primary care as usual). Physiatrists will give explanations about underlying pain mechanisms (hurt does not mean harm) and will encourage patients to be physically active and to restart functional daily activities.

\section{PHYSIOTHERAPY PRACTICES \& THERAPISTS}

Eight physiotherapy practices located in Maastricht and the surrounding area will be selected to provide physiotherapy interventions. To minimise the risk for contamination between the interventions, four physiotherapy practices will provide the Back on Track intervention and four physiotherapy practices will provide primary care as usual. Selection criteria for physiotherapy practices include appropriate facilities, willingness to participate, and motivation to provide a biopsychosocial intervention in case the practice is assigned to the Back on Track intervention. Each physiotherapy practise will designate one or two physiotherapists to provide physiotherapy interventions within the current study. No specific criteria are established for this selection (e.g. years of experience, skills or knowledge of cognitive behavioural interventions). Physiotherapists providing care as usual will not receive information about the content of the Back on Track intervention.

\section{BACK ON TRACK INTERVENTION}

The Back on Track intervention is based on principles well known from literature and used in cognitive behavioural interventions as for example Graded Activity and Graded exposure. ${ }^{16,17}$ Graded Activity uses an operant conditioning approach in order to gradually, time-contingently, increase the activity 
level of patients. Graded Activity has proven to be significantly effective in improving pain and functional disability as compared to minimal interventions or no interventions ${ }^{18}$ and significantly more effective in improving occupational functioning than regular care (varying from rest and analgesics to physiotherapy). ${ }^{16,19}$ Graded Exposure uses a classical conditioning approach and is used to expose patients to fearful activities stimulating patients to recognise the inconsistency between their unrealistic thoughts about fearful activities and the actual consequences of these fearful activities. Graded Exposure has been shown effective in improving pain-related fear, pain catastrophizing ${ }^{20,21}$ and has been shown equally effective in improving functional disability as Graded Activity. ${ }^{22,23}$

The Back on Track intervention consists of four individual sessions (30 minutes) and eight group sessions (60 minutes; Table 1).

Table 1 Time schedule of the Back on Track intervention

\begin{tabular}{llr}
\hline Session & Content & Min. \\
\hline Individual session 1 & History taking, screening & 30 \\
Individual session 2 & Pain education, defining influencing (bio)psychosocial factors & 30 \\
Individual session 3 & Goal setting (SMART) & 30 \\
Group session 1-8 & Theme 1. Pain \& physical activity (elements of Graded Activity) & $2 \times 60$ \\
& Theme 2. Pain \& social factors & $2 \times 60$ \\
& Theme 3. Pain \& cognitions (elements of Graded Exposure) & $2 \times 60$ \\
Individual session 4 & Theme 4. Low back pain: Myth or fact? & $2 \times 60$ \\
& Evaluation with physiotherapists & 30 \\
\hline
\end{tabular}

Back on Track physiotherapists will receive a treatment protocol with detailed information about each session and patients will receive a workbook with explanations, illustrations and home assignments. It has been shown that explanations by therapists during therapy in conjunction with written explanations and illustrations provided in booklets are effective in changing pain perceptions and health status. ${ }^{24}$ Patients seem positive about receiving general information about pain as it improves knowledge and understanding of the complexity of the pain problem. ${ }^{24,25}$

Individual sessions of the Back on Track intervention mainly focus on history taking (session 1), pain education (e.g. definition of pain, differences between acute and chronic pain) and exploration of patient-specific psychosocial factors (session 2), and goal setting for most restricted functional activities (session 3). Thereafter, groups will be provided twice a week and will focus each week on one theme in particular. Themes include pain \& physical activity (theme 1), pain \& psychological factors (theme 2), pain \& social factors (theme 3 ) and low back pain: myth or fact? (theme 4). The first group session of each theme will be an education-based session aiming to gain insight in the role of (bio-) psychosocial factors and potential ways to cope with these factors. The second session of each theme will be a more physically active based session in order to extract patients from their comfort zone, to increase confidence in their own body, to have 
pleasure in being active and to become more physically/functionally active. In all group sessions, elements of Graded Activity and Graded Exposure will be used to change cognitions, coping styles and to improve functional disability levels.

Overall, the main objectives of the Back on Track intervention are:

- To gain insight in pain mechanisms and the transition from acute to chronic pain;

- To gain insight in the role of physical activity levels, cognitions and social life;

- To set patient-specific goals and to improve activity levels;

- To gain insight in potential catastrophizing thoughts and fear-avoidance behaviours;

- To stimulate active coping styles and self-management strategies;

In case a patient, after completion of the Back on Track intervention, was not able to improve his/her functional disability level or is not capable in translating learned objectives towards daily settings, he/she will be referred back to his/her physiatrist. The physiatrist will evaluate the current status of the patient and, if necessary, will discuss remaining questions and repeat previously provided medical education. Finally, the necessity of additional care will be determined.

Prior to the study, Back on Track physiotherapists will be educated by specialised cognitive-behavioural therapists and experienced physiotherapists within three meetings of four hours each. The educational programme aims to improve knowledge regarding pain and CLBP, the influence of biopsychosocial factors on CLBP, the relevance of cognitive-behavioural principles, and practical implementation of the Back on Track intervention. It is assumed that multiple educational meetings will enable physiotherapists to apply knowledge into practice and to discuss experiences in subsequent meetings. Additionally, physiotherapists will receive supervision from members of the educational team throughout the intervention. Supervision will include individual feedback whenever necessary and intervision in order to improve the physiotherapists' skills and knowledge.

One additional aim of the Back on Track intervention is to improve communication between primary and secondary care by using a specific safeguarded software programme. Physiatrists and physiotherapists of the Back on Track intervention will use this software programme in order to exchange patient-specific medical information. Exchanging information will create transparency in defined diagnoses, treatment strategies and findings, and might lead to less time-consuming health care since professionals will be able to share medical information.

\section{PRIMARY CARE AS USUAL}

Primary care as usual will be provided by physiotherapists according to their current knowledge and the Dutch profession-specific guideline for LBP. ${ }^{2}$ Therapy will not be protocolled, but will be restricted to a maximum of twelve individual sessions (30 minutes) within eight weeks in order to preserve from infinite number of treatments and to keep the amount of attention between interventions more equal and comparable. To gain insight in the provided therapy, physiotherapists will record the number, content and time spent on every session for each patient specifically. 


\section{OUTCOME MEASURES}

Measurements will be performed pre-treatment, post-treatment, at 3 and 12 months follow-up, unless stated otherwise (Table 2).

\section{Primary outcome}

Functional disability level will be determined using the Quebec Back Pain Disability Scale (QBPDS). Improving functional disability is the key outcome of most physiotherapy interventions for CLBP. ${ }^{26}$ The QBPDS consists of 20 items, each scored from 0 (not difficult at all) to 5 (unable to perform). Maximum score (100) therefore represents maximally functionally disabled. Since the QBPDS has a sufficient scale range to detect reliable improvements or deteriorations and is proved to be among the most reliable scale for functional disability, ${ }_{1}{ }^{7}$ the QBPDS is the main study parameter of this study. The QBPDS proved to have high internal consistency, good test-retest reliability, sufficient responsiveness and validity. ${ }^{27-29}$

Table 2 Outcome measures of the Back on Track study

\begin{tabular}{|c|c|c|}
\hline Domain & Measures & Time points* \\
\hline \multicolumn{3}{|l|}{ Primary outcome } \\
\hline Disability & Quebec Back Pain Disability Scale & $\mathrm{T} 0, \mathrm{~T} 1, \mathrm{~T} 2, \mathrm{~T} 3$ \\
\hline \multicolumn{3}{|l|}{ Secondary outcomes } \\
\hline Pain intensity & Numeric Rating Scale & $\mathrm{T} 0, \mathrm{~T} 1, \mathrm{~T} 2, \mathrm{~T} 3$ \\
\hline Pain catastrophizing & Pain Catastrophizing Scale & $\mathrm{T} 0, \mathrm{~T} 1, \mathrm{~T} 2, \mathrm{~T} 3$ \\
\hline Anxiety and depression & Hospital Anxiety and Depression Scale & $\mathrm{T} 0, \mathrm{~T} 1, \mathrm{~T} 2, \mathrm{~T} 3$ \\
\hline Pain-related fear & Tampa Scale of Kinesiophobia & $\mathrm{T} 0, \mathrm{~T} 1, \mathrm{~T} 2, \mathrm{~T} 3$ \\
\hline Self-efficacy & Pain Self-Efficacy Questionnaire & $\mathrm{T} 0, \mathrm{~T} 1, \mathrm{~T} 2, \mathrm{~T} 3$ \\
\hline Global perceived effect & Global Perceived Effect & $\mathrm{T} 1, \mathrm{~T} 2, \mathrm{~T} 3$ \\
\hline Credibility and expectancy & Credibility and Expectancy Questionnaire & Directly after session 1 \\
\hline \multicolumn{3}{|l|}{ Economic evaluation } \\
\hline Quality of life & EuroQol-5D & $\mathrm{T} 0, \mathrm{~T} 1, \mathrm{~T} 2, \mathrm{~T} 3$ \\
\hline Cost diary & $\begin{array}{l}\text { Trimbos and iMTA questionnaire on Costs } \\
\text { associated with Psychiatric illness }\end{array}$ & $\mathrm{T} 0, \mathrm{~T} 1, \mathrm{~T} 2, \mathrm{~T} 3$ \\
\hline \multicolumn{3}{|l|}{ Process evaluation } \\
\hline Protocol adherence & Voice recordings Back on Track intervention & All therapy sessions \\
\hline
\end{tabular}

\footnotetext{
* T0: baseline;T1: post-treatment; T2: 3 months follow-up;T3: 12 months follow-up
} 


\section{Secondary outcome (effect evaluation)}

Pain intensity will be measured using the Numeric Rating Scale (NRS). The NRS is a quick and easy to administer scale to measure the patient's current level of pain intensity, the highest and lowest level of pain intensity last week and the level of pain intensity experienced during the last night. The questionnaire can be rated from 0 (no pain) to 10 (worst imaginable pain). The average pain intensity will finally be calculated.

Pain catastrophizing will be determined using the Pain Catastrophizing Scale (PCS). ${ }^{30}$ The PCS consists of 13 definitions regarding thoughts and feelings when experiencing pain. The definitions can be rated on a 5-point scale from 0 (not at all) to 4 (all the time). Total score ranges from 0-52 indicating higher levels of catastrophizing thoughts and feelings at higher scores. The Dutch version of the PCS appears to be valid and highly reliable. .1,32 $^{1}$

Anxiety and depression will be determined by the Hospital Anxiety and Depression Scale (HADS). The HADS consists of 7 anxiety-related questions and 7 depression-related questions. All items in both subscales can be rated from 0 (not at all) to 3 (most of the time). Total score ranges from 0-21 on each subscale in which a higher score reflects higher distress. The HADS questionnaire appears to have adequately psychometric properties (e.g. reliability and validity). ${ }^{33}$

Pain-related fear will be assessed by the Tampa Scale of Kinesiophobia (TSK). This 17-itemed questionnaire can be rated from 1 (totally disagree) to 4 (totally agree). Total score ranges from 17-68, with higher scores reflecting higher fear of movement or (re)injury. The Dutch version of the TSK appeared to be reliable and valid. ${ }^{34,35}$

Pain self-efficacy will be measured using the Pain Self-Efficacy Questionnaire (PSEQ). Ten questions will be rated on a 7-point Likert scale from 0 (not at all confident) to 6 (completely confident). This questionnaire reflects the confidence of patients regarding the ability to perform activities in the experience of pain. Total score ranges from 0-60, representing stronger self-efficacy beliefs at higher scores. Psychometric properties of the PSEQ are excellent. ${ }^{36}$

Quality of Life will be measured by the EuroQol-5D (EQ-5D). ${ }^{37}$ The EQ-5D consists of five dimensions: mobility, self-care, usual activities, pain/discomfort and anxiety/depression. The questionnaire proved to be a valid and responsive instrument to assess quality of life in patients with CLBP. ${ }^{38}$

Global perceived effect of the treatment will be determined using the Global Perceived Effect (GPE) questionnaire. The GPE reports the patient's opinion about two aspects of the treatment: recovery and satisfaction. Both questions use a 7-point Likert scale ranging from 1-7 ("completely recovered" to "worse than ever", and "satisfied" to "very dissatisfied"). The GPE questionnaire appears to have excellent reproducibility. ${ }^{39}$ The GPE will be measured at post-treatment, and 3 and 12 months of follow-up.

\section{Potential moderator and prognostic factor}

Credibility and expectancy of the treatment will be measured with the Dutch Credibility and Expectancy Questionnaire (CEQ) developed by Smeets and colleagues. ${ }^{40}$ The Dutch version of the CEQ proved to have high internal consistency. An adapted version of the Dutch CEQ will be used 
for this study and will consist of two subscales: 5 items are related to credibility and expectancy, and 6 items are related to the success the patient is expected to perceive. The questionnaire uses a rating scale of 1-9, ranging from "not at all" to "very much", reflecting higher credibility/ expectancy at higher scores. The CEQ will be completed directly after the first therapy session.

\section{Secondary outcome (economic evaluation)}

The economic evaluation will be carried out from a societal perspective. To assess economic consequences, indirect and direct healthcare costs will be measured. In order to calculate intervention costs, therapists will document the number of treatments and the duration of each treatment for each patient. Health care costs will be measured with the Trimbos/iMTA questionnaire on Costs associated with Psychiatric illness (TiC-P). ${ }^{41}$ The questionnaire consists of 15 items and includes questions about the number of consultations, use of medication, hospital visits and other medical/social care over the past three months. It represents the patient's work and household activities. ${ }^{42}$ In addition, productivity losses will be assessed with the second part of the TiC-P including 11 items of the Short-Form Health and Labour Questionnaire (SF-HLQ). ${ }^{43}$

\section{Secondary outcome (process evaluation)}

Treatment fidelity will be measured to investigate whether treatment outcomes are related to the Back on Track intervention. Recommendations from existing guidelines will be used to measure treatment fidelity with regard to the Back on Track intervention. ${ }^{44,45}$ Physiotherapists of the Back on Track intervention will be asked to report treatment location, to report the number of individual/group sessions, and to make audio recordings of each therapy session in order to assess protocol adherence (see also section process evaluation). Care as usual physiotherapists will only be asked to report treatment location, treatment duration and treatment content of each session in order to get insight in the treatment strategies of primary care as usual.

\section{PROCEDURE}

Both male and female patients with CLBP will be recruited and assessed for eligibility by physiatrists at the department of rehabilitation medicine at MUMC+, the Netherlands. As mentioned previously, prior to this consultation a set of questionnaires will be completed (e.g., socio-demographic questionnaire, SF-36, Pain Disability Index (PDI), PCS, NRS, and HADS). Physiatrists will use scores on the abovementioned questionnaires and their clinical expertise to determine the influence of psychosocial factors and the associated disability level of patients. Eligible patients will receive oral and written information about the study and will give written consent to transfer general contact information to the research team (Figure 1).

One week after receiving written consent, patients will be contacted by phone and invited for a personal intake session by a researcher at Maastricht University (UM). Remaining questions will be answered and written consent to participate in the study will be provided during this intake session. The written consent procedure also includes a confirmation of a request to use 
already available results of questionnaires completed prior to the consultation at the department of rehabilitation medicine (socio-demographic questionnaire, SF-36, PDI, PCS, NRS and HADS). By using these outcomes from patient care assessment batteries, duplicate completion of questionnaires, requiring extra load for patients, will be prevented. Outcomes are expected to remain unchanged within this small time frame. Finally, remaining questionnaires (baseline measures; T0) will be completed electronically via a web-based software programme in a private room at the UM without attendance of any member of the research team.

\section{Randomisation}

Central randomisation (1:1) will be executed by an assistant investigator via a computerised random number generator using block-randomisations with random block sizes of 4,6 and 8 . No stratification will be used. A randomisation list will be prepared by the assistant investigator which will be accessible only for the assistant investigator. The assistant investigator will assign the participant to one of the two interventions and will inform both participant and therapist about the allocation. The physiotherapist will subsequently contact the patient to start the therapy.

\section{Blinding}

This study uses a double-blind design with data-analysts and patients blinded to treatment allocation. Patients will receive general information about the allocated practice (i.e. location etc.), but not treatment allocation. However, patients will presumably recognise the allocated therapy to which they are randomised. In addition, patients will also be blinded for the study hypotheses. Physiatrists and physiotherapists cannot be blinded for treatment allocation because they are involved in practical implementation of the therapy.

\section{Monitoring}

Clinical Trial Centre Maastricht (CTCM), the Netherlands, will monitor protocol fidelity of the trial. Monitoring will comprise one initiation visit, 3 interim visits and one close-out visit. Accuracy and completeness of reported trial data and compliance with the approved protocol will be assessed.

\section{Trial status}

Ethical approval of the study was provided in July 2014. Currently the data collection is ongoing until November 2016 and data analysis will be executed from October until June 2017. Final results of the study will be available in June 2017. 


\section{DATA ANALYSIS}

\section{Effect evaluation}

Data analyses will be executed using IBM SPSS Statistics 22. Analyses will be performed using an intention-to-treat principle i.e. all patients will keep their allocated therapy they were randomised to, regardless of poor compliance or withdrawal. Due to the longitudinal design of the study including repeated measures at different time points, linear mixed models will be used to evaluate the differences in outcomes over one year between the primary care Back on Track intervention and primary care as usual. The model will be adjusted for the baseline value and will use the follow-up measurement as dependent variable.

\section{Economic evaluation}

Intervention costs will be calculated by multiplying the number of treatments with cost prices for physiotherapy treatments set by health insurance company CZ. Cost prices of additional medical consumption (TiC-P) will be calculated using an updated version of the Dutch manual for costs analysis in healthcare research. ${ }^{46} \mathrm{All}$ costs will be presented in Euros. The human capital approach ( $\mathrm{HCA}$ ) will be used to calculate productivity costs. Mean total costs of the therapy groups will eventually be compared. In addition, cost-effectiveness analysis and cost-utility analysis will be performed. For the cost-effectiveness analysis, an incremental cost-effectiveness ratio (ICER) will be calculated in which total costs will be weighed against disability levels (QBPDS). With regard to the cost-utility analysis, mean total costs will be weighed against mean health utility, i.e. comparing cost per Quality Adjusted Life Years (QALY) gained. Utility will be calculated from EQ-5D scores for every assessment (T0, T1, T2 and T3) using the Dolan algorithm. ${ }^{47}$ Gains in QALY over one year will be calculated using the area under the curve.

Additionally, bootstrapping will be used to explore sample uncertainty (5000 replications). These bootstrap simulations will be conducted to quantify the uncertainty around the ICER, yielding information about the joint distribution of cost and effect differences between the interventions. Furthermore, the results of this study will also be depicted in a cost-effectiveness acceptability curve (CEAC), representing the uncertainty around the cost-effectiveness of the Back on Track intervention compared to primary care as usual.

\section{Process evaluation}

Treatment fidelity (protocol adherence) will be measured using audiotapes and a modified version of the protocol used by Leeuw and colleagues. ${ }^{48}$ The protocol will include most important and essential topics as well as prohibited topics per session. A random selection of the audiotapes will be drawn according to the method used in a study of Leeuw and colleagues. ${ }^{48}$ Adherence will be regarded sufficient in case $70 \%$ of the required treatment elements are provided during the treatment. Two independent raters blinded to the study hypotheses will assess treatment fidelity 
to the Back on Track intervention. The homogeneity of the ratings on the self-developed instrument between the two independent investigators will be assessed using inter-rater reliability (Cohen's kappa). Additional data with regard to the process evaluation will be analysed descriptively.

\section{Sample size calculation}

Sample size calculation was based on a study of Smeets et al. who investigated the improvement in functional disability after a physical activity programme (baseline - post-treatment) in patients with CLBP using the Roland-Morris Disability Questionnaire (RDQ, mean baseline $14.15 \pm 3.70){ }^{49}$ This study investigated also the improvement in QBPDS after a physical activity programme (baseline-post-treatment) which has not been reported in the published paper. The QBPDS at baseline was $44.72 \pm 13.96$ and decreased with $3.0 \pm 11.21$ post-treatment. Due to the fact the Back on Track study also uses the QBPDS as primary outcome and QBPDS scores before and after treatment of the study of Smeets et al. were available, sample size calculation is based on QBPDS scores of the study of Smeets et al. In order to detect a minimal clinically important difference at 12 months follow-up between the new Back on Track intervention and primary care as usual in the present study, a difference of improvement of at least 15\% on the QBPDS between the two interventions was chosen. Calculating a 15\% improvement from the mean baseline QBPDS score (44.72) of the study of Smeets et al., this results in a difference of improvement of 7 points. Next, correlations were calculated from the data of Smeets et al. between baseline measurements and post-treatment measurements ( $\rho=0.746$ ) to adjust for repeated measures $(r=3)$. Taken into account a 2-tailed test with a significance level of 0.05 , a power of $80 \%$ and drop-out rate of $20 \%$, a sample size of 43 per group will be necessary for the present study.

\section{DISCUSSION}

Existing guidelines present general recommendations for biopsychosocial interventions in patients with CLBP. 2,11 Such biopsychosocial interventions are primarily offered in expensive multicentre rehabilitation teams to patients with complex psychosocial factors and high levels of functional disability. Evidence about whether a biopsychosocial intervention would also be effective in patients treated in primary care (i.e. having less complex psychosocial factors and lower functional disability levels) is still scarce. In addition, no conclusive evidence is available about "which primary care intervention works best" for this specific subgroup of patients with CLBP treated in primary care. Due to this reason, it is the aim of the study to compare the effectiveness of a biopsychosocial primary care intervention to primary care as usual (physiotherapy) in a subgroup of patients who are usually treated in primary care settings and experience non-complex (but present) psychosocial factors and low to moderate functional disability levels. For this study, a new biopsychosocial primary care intervention 'Back on Track' has been developed which is based on biopsychosocial approaches well-known from literature and used in multidisciplinary pain rehabilitation settings. 
The internal strength of the Back on Track intervention is the focus on multiple relevant factors such as physical, cognitive and environmental factors, which might have an influence on the development and/or maintenance of CLBP. It is expected that our subgroup of patients will benefit from this intervention since patients experience non-complex but present psychosocial factors. By addressing various influencing factors and by providing different methods to counteract these influencing factors (e.g. elements of Graded Activity and Graded Exposure), patients will discover which approach suits them best. Patients will be encouraged to apply elements of both methods actively in their situation in order to improve their daily life functioning. A workbook with detailed information and illustrations will stimulate active participation of patients during the intervention and will enable them to add important notes whenever desired and to reread information in case new episodes of low back pain occur in future.

A major strength of the study is the double-blinded RCT-design of the study. Blinded treatment allocation for patients and data-analysts, as well as the concealed allocation procedure executed by an assistant investigator will reduce the chance of confounding and bias. Furthermore, the trial will be monitored by an independent monitor to assess protocol deviations as well.

An additional strength of this study is the selection of patients by experienced medical practitioners instead of for example a general practitioner, a physiotherapist or a selection based on a questionnaire. A medical practitioner specialised in the evaluation of psychosocial profiles, such as the physiatrist in the Dutch health care system, is expected to be able to adequately select a homogeneous group of patients. Selecting a homogeneous group of patients is recommended by several authors since this leads to less individual variation what is expected to result in a better effect of an intervention. 6.7

This study aims to keep referral and organisational procedures similar to those used in usual practise nowadays (determining psychosocial profiles, referral to appropriate therapy settings). By doing so, only patients with CLBP who normally receive therapy in primary care will be included what will lead to a better reflection of current health care as well as a selection of a homogeneous group of patients with corresponding psychosocial profiles. Furthermore, no specific criteria will be used to select physiotherapists in both interventions. It is expected that all these pragmatic procedures will lead to an overall better reflection of the feasibility (and effectiveness) of the new biopsychosocial Back on Track intervention in usual primary care setting $5^{50}$ what might subsequently result in conclusions that will be easier to implement in usual care.

Physiotherapists providing the Back on Track intervention will be offered an educational programme and treatment protocol, and will receive supervision during the study. Recommendations from existing guidelines to enhance treatment fidelity as well as to measure it will be used for this study. ${ }^{44,45}$ Measuring treatment fidelity will give useful information with regard to the implementation of the intervention and the degree effects of the study are actually related to the intervention.

One limitation of the study might be the use of self-reported questionnaires. Even though validated self-reported questionnaires have remarkable advantages with regard to psychometric qualities, time-management and financial aspects, they might lead to socially desirable answers 
resulting in response bias. This study will try to minimise this aspect by offering electronic questionnaires enabling patients to complete questionnaires at home without the attendance of physiotherapists or members of the research team.

Another possible limitation might also be the fact that primary care as usual will not be standardised in a protocol what might result in unequal attention and treatment strategies between patients in the usual care-group. However, in order to minimise unequal attention, and to prevent from endless treatments, care as usual will be restricted to a maximum of twelve treatments (30 minutes each) within eight weeks.

In summary, the results of this study will provide practical recommendations for primary care interventions (effectiveness study) as well as practical recommendations for policy and health plan decisions (cost-effectiveness study) in order to support the management of CLBP in patients who normally receive treatments in primary care (experiencing low to moderate levels of disability and non-complex but present psychosocial factors).

\section{ACKNOWLEDGEMENTS}

We acknowledge Paul Willems, Frans Abbink, Albère Köke and Marlies den Hollander for their contribution to the development of the Back on Track intervention and the content and implementation of the educational programme. Furthermore, we acknowledge the Department of Rehabilitation in medicine MUMC+, Spine Centre MUMC+ and Fy'net Collaboration for the management of the trial. The Back on Track study is sponsored by Adelante, Centre of Expertise in Rehabilitation and Audiology Hoensbroek, the Netherlands; the Province of Limburg and CZ Foundation. 


\section{REFERENCES}

1. Picavet HS, Schouten JS. Musculoskeletal pain in the Netherlands: prevalences, consequences and risk groups, the DMC(3)-study. Pain. Mar 2003;102(1-2):167-178.

2. Staal JB, M. HEJ, Heijmans M, et al. KNGF-richtlijn Lage-rugpijn. Amersfoort: Drukkerij De Gans;2013.

3. van Tulder MW, Koes BW, Bouter LM. A cost-of-illness study of back pain in The Netherlands. Pain. 1995;62(2):233-240.

4. Picavet H. Aspecifieke lage rugklachten: omvang en gevolgen. 2005/2007 CvP-eZ. 2005/2007.

5. Koes BW, van Tulder MW, Poos MJJC. Neemt het aantal mensen met nek- en rugklachten toe of af? In: Volksgezondheid NK, ed. Bilthoven: RIVM; 2014.

6. Jellema P, van der Horst HE, Vlaeyen JW, Stalman WA, Bouter LM, van der Windt DA. Predictors of outcome in patients with (sub)acute low back pain differ across treatment groups. Spine. Jul 1 2006;31(15):1699-1705.

7. George SZ, Fritz JM, Bialosky JE, Donald DA. The effect of a fear-avoidance-based physical therapy intervention for patients with acute low back pain: results of a randomized clinical trial. Spine. Dec 1 2003;28(23):2551-2560.

8. Kamper SJ, Apeldoorn AT, Chiarotto A, et al. Multidisciplinary biopsychosocial rehabilitation for chronic low back pain. The Cochrane database of systematic reviews. Sep 2 2014;9:CD000963.

9. Monticone M, Ferrante S, Rocca B, Baiardi P, Farra FD, Foti C. Effect of a long-lasting multidisciplinary program on disability and fear-avoidance behaviors in patients with chronic low back pain: results of a randomized controlled trial. The Clinical journal of pain. Nov 2013;29(11):929-938.

10. van Middelkoop M, Rubinstein SM, Kuijpers T, et al. A systematic review on the effectiveness of physical and rehabilitation interventions for chronic non-specific low back pain. European spine journal : official publication of the European Spine Society, the European Spinal Deformity Society, and the European Section of the Cervical Spine Research Society. Jan 2011;20(1):19-39.

11. Airaksinen O, Brox Jl, Cedraschi C, et al. Chapter 4. European guidelines for the management of chronic nonspecific low back pain. European spine journal : official publication of the European Spine Society, the European Spinal Deformity Society, and the European Section of the Cervical Spine Research Society. Mar 2006;15 Suppl 2:S192-300.

12. Swinkels IC, van den Ende CH, van den Bosch W, Dekker J, Wimmers RH. Physiotherapy management of low back pain: does practice match the Dutch guidelines? The Australian journal of physiotherapy. 2005;51(1):35-41.

13. Lamb SE, Hansen Z, Lall R, et al. Group cognitive behavioural treatment for low-back pain in primary care: a randomised controlled trial and cost-effectiveness analysis. Lancet. Mar 13 2010;375(9718):916-923.

14. Lamb SE, Mistry D, Lall R, et al. Group cognitive behavioural interventions for low back pain in primary care: extended follow-up of the Back Skills Training Trial (ISRCTN54717854). Pain. Feb 2012;153(2):494501.

15. van der Roer N, van Tulder M, Barendse J, Knol D, van Mechelen W, de Vet H. Intensive group training protocol versus guideline physiotherapy for patients with chronic low back pain: a randomised controlled trial. European spine journal : official publication of the European Spine Society, the European Spinal Deformity Society, and the European Section of the Cervical Spine Research Society. Sep 2008;17(9):1193-1200. 
16. Lindstrom I, Ohlund C, Eek C, et al. The effect of graded activity on patients with subacute low back pain: a randomized prospective clinical study with an operant-conditioning behavioral approach. Phys Ther. 1992;72(4):279-290; discussion 291-273.

17. Vlaeyen JW, Linton SJ. Fear-avoidance and its consequences in chronic musculoskeletal pain: a state of the art. Pain. Apr 2000;85(3):317-332.

18. Macedo LG, Smeets RJ, Maher CG, Latimer J, McAuley JH. Graded activity and graded exposure for persistent nonspecific low back pain: a systematic review. Physical therapy. Jun 2010;90(6):860-879.

19. Staal JB, Hlobil H, Twisk JW, Smid T, Koke AJ, van Mechelen W. Graded activity for low back pain in occupational health care: a randomized, controlled trial. Annals of internal medicine. Jan 20 2004;140(2):7784.

20. Vlaeyen JW, de Jong J, Geilen M, Heuts PH, van Breukelen G. The treatment of fear of movement/(re) injury in chronic low back pain: further evidence on the effectiveness of exposure in vivo. The Clinical journal of pain. Jul-Aug 2002;18(4):251-261.

21. Boersma K, Linton S, Overmeer T, Jansson M, Vlaeyen J, de Jong J. Lowering fear-avoidance and enhancing function through exposure in vivo. A multiple baseline study across six patients with back pain. Pain. Mar 2004;108(1-2):8-16.

22. George SZ, WittmerVT, Fillingim RB, Robinson ME. Comparison of graded exercise and graded exposure clinical outcomes for patients with chronic low back pain. The Journal of orthopaedic and sports physical therapy. Nov 2010;40(11):694-704.

23. Leeuw M, Goossens ME, van Breukelen GJ, et al. Exposure in vivo versus operant graded activity in chronic low back pain patients: results of a randomized controlled trial. Pain. Aug 15 2008;138(1):192-207.

24. Nijs J, Paul van Wilgen C, Van Oosterwijck J, van Ittersum M, Meeus M. How to explain central sensitization to patients with 'unexplained' chronic musculoskeletal pain: practice guidelines. Manual therapy. Oct 2011;16(5):413-418.

25. Moseley GL, Nicholas MK, Hodges PW. A randomized controlled trial of intensive neurophysiology education in chronic low back pain. The Clinical journal of pain. Sep-Oct 2004;20(5):324-330.

26. Delitto A. Are measures of function and disability important in low back care? Physical therapy. May 1994;74(5):452-462.

27. Davidson M, Keating JL. A comparison of five low back disability questionnaires: reliability and responsiveness. Phys Ther. 2002;82(1):8-24.

28. Schoppink LE, van Tulder MW, Koes BW, Beurskens SA, de Bie RA. Reliability and validity of the Dutch adaptation of the Quebec Back Pain Disability Scale. Phys Ther. 1996;76(3):268-275.

29. Smeets R, Koke A, Lin CW, Ferreira M, Demoulin C. Measures of function in low back pain/disorders: Low Back Pain Rating Scale (LBPRS), Oswestry Disability Index (ODI), Progressive Isoinertial Lifting Evaluation (PILE), Quebec Back Pain Disability Scale (QBPDS), and Roland-Morris Disability Questionnaire (RDQ). Arthritis care \& research. Nov 2011;63 Suppl 11:S158-173.

30. Sullivan MJ, Bishop SR, Pivik J. The Pain Catastrophizing Scale: Development and validation. Psychological Assessment. 1995;7:524-532.

31. Crombez G, Eccleston C, Baeyens F, Eelen P. When somatic information threatens, catastrophic thinking enhances attentional interference. Pain. 1998a;75(2-3):187-198.

32. Crombez G, Vlaeyen JW, Heuts PH, Lysens R. Pain-related fear is more disabling than pain itself: evidence on the role of pain-related fear in chronic back pain disability. Pain. 1999a;80(1-2):329-339. 
33. Hansson M, Chotai J, Nordstom A, Bodlund O. Comparison of two self-rating scales to detect depression: HADS and PHQ-9. The British journal of general practice : the journal of the Royal College of General Practitioners. Sep 2009;59(566):e283-288.

34. Vlaeyen JW, Kole-Snijders AM, Boeren RG, van Eek H. Fear of movement/(re)injury in chronic low back pain and its relation to behavioral performance. Pain. Sep 1995;62(3):363-372.

35. Goubert L, Crombez G, Vlaeyen J, van Damme S, van den Broeck A, van Houdenhove B. De Tampa Schaal voor Kinesiofobie: Psychometrische karakteristieken en normering (The Tampa scale Kinesiophobia: psychometric properties and norms). Gedrag Gezond. 2000;28:54-62.

36. Nicholas MK. The pain self-efficacy questionnaire: Taking pain into account. European journal of pain. Feb 2007;11(2):153-163.

37. EuroQol G. EuroQol—a new facility for the measurement of health-related quality of life. Health policy. Dec 1990;16(3):199-208.

38. Soer R, Reneman MF, Speijer BL, Coppes MH, Vroomen PC. Clinimetric properties of the EuroQol-5D in patients with chronic low back pain. The spine journal : official journal of the North American Spine Society. Nov 2012;12(11):1035-1039.

39. Kamper SJ, Ostelo RW, Knol DL, Maher CG, de Vet HC, Hancock MJ. Global Perceived Effect scales provided reliable assessments of health transition in people with musculoskeletal disorders, but ratings are strongly influenced by current status. Journal of clinical epidemiology. Jul 2010;63(7):760-766 e761.

40. Smeets RJ, Beelen S, Goossens ME, Schouten EG, Knottnerus JA, Vlaeyen JW. Treatment expectancy and credibility are associated with the outcome of both physical and cognitive-behavioral treatment in chronic low back pain. The Clinical journal of pain. May 2008;24(4):305-315.

41. Hakkaart- van Roijen L. Manual Trimbos/iMTA questionnaire for Costs associated with Psychiatric illness (TiC-P). Rotterdam: Institute for Medical Technology Assessment, Erasmus University Rotterdam;2010.

42. Bouwmans C, De Jong K, Timman R, et al. Feasibility, reliability and validity of a questionnaire on healthcare consumption and productivity loss in patients with a psychiatric disorder (TiC-P). BMC health services research. 2013;13:217.

43. Hakkaart- van Roijen L, Bouwmans CAM. Short Form-Health and Labour Questionnaire (SF-HLQ). Rotterdam: Institute for Medical Technology Assessment. Erasmus Universitair Medisch Centrum Rotterdam;2007.

44. Bellg AJ, Borrelli B, Resnick B, et al. Enhancing treatment fidelity in health behavior change studies: best practices and recommendations from the NIH Behavior Change Consortium. Health psychology : official journal of the Division of Health Psychology, American Psychological Association. Sep 2004;23(5):443-451.

45. Saunders RP, Evans MH, Joshi P. Developing a process-evaluation plan for assessing health promotion program implementation: a how-to guide. Health promotion practice. Apr 2005;6(2):134-147.

46. Hakkaart- van Roijen L, Tan LSS, Bouwmans CAM. Handleiding voor kostenonderzoek, methoden en standaard kostprijzen voor economische evaluaties in de gezondheidszorg. Geactualiseerde versie 2010. College voor Zorgverzekeringen (CVZ). 2010.

47. Dolan P. Modeling valuations for EuroQol health states. Medical care. Nov 1997;35(11):1095-1108.

48. Leeuw M, Goossens ME, de Vet HC, Vlaeyen JW. The fidelity of treatment delivery can be assessed in treatment outcome studies: a successful illustration from behavioral medicine. Journal of clinical epidemiology. Jan 2009;62(1):81-90. 
49. Smeets RJ, Vlaeyen JW, Hidding A, et al. Active rehabilitation for chronic low back pain: cognitive-behavioral, physical, or both? First direct post-treatment results from a randomized controlled trial [ISRCTN22714229]. BMC musculoskeletal disorders. 2006;7:5.

50. Thorpe KE, Zwarenstein M, Oxman AD, et al. A pragmatic-explanatory continuum indicator summary (PRECIS): a tool to help trial designers. CMAJ : Canadian Medical Association journal = journal de I'Association medicale canadienne. May 12 2009;180(10):E47-57. 


\section{APPENDIX}

APPENDIX I CLASSIFICATION SYSTEM AND HEALTH CARE POLICY IN THE NETHERLANDS FOR PATIENTS WITH CHRONIC LOW BACK PAIN BASED ON THE PSYCHOSOCIAL PROFILE AND THE LEVEL OF FUNCTIONAL DISABILITY

\begin{tabular}{clll}
\hline Classification & Psychosocial factors & Disability level & Health care policy/therapy setting \\
\hline 1 & Not present & - & Primary care (general practitioner) \\
2 & Present but not complex & Low & Primary care (physiotherapist) \\
3 & Present and slightly complex & Moderate & Secondary care (multidisciplinary) \\
4 & Present and complex & High & Secondary/tertiary care (multidisciplinary) \\
\hline
\end{tabular}





\title{
Chapter 5
}

Biopsychosocial primary care and physiotherapy as usual show no differences in effects in patients with chronic low back pain: results of a randomised controlled trial

\author{
Reni van Erp \\ Ivan Huijnen \\ Ton Ambergen \\ Jeanine Verbunt \\ Rob Smeets
}





\section{ABSTRACT}

Question: What is the effect of a biopsychosocial primary care intervention (Back on Track) as compared to primary care physiotherapy as usual in decreasing the level of disability at post treatment and 3 months follow-up in patients with chronic low back pain (CLBP) experiencing low complex psychosocial complaints?

Design: A double-blind multicentre randomised controlled trial using intention-to-treat analysis.

Participants: Twenty-five patients ( $\geq 18$ yr.) with non-specific CLBP ( $\geq$ 12 wk.) experiencing low complex psychosocial complaints and a low to moderate level of disability.

Intervention: A biopsychosocial primary care physiotherapy intervention (4 individual and 8 group sessions) versus primary care physiotherapy as usual (maximally 12 individual sessions).

Outcome measures: Primary outcome was functional disability (Quebec Back Pain Disability Scale) at post-treatment and 3 months follow-up. Secondary measures were anxiety, depression, catastrophizing, pain intensity, kinesiophobia, self-efficacy, patient's global perceived effect. Effects were analysed using linear mixed model analysis.

Results: No significant differences in functional disability were found between the Back on Track intervention and physiotherapy as usual at post-treatment (mean difference $0.10,95 \% \mathrm{Cl}-12.9$ to 13.1 ) and 3 months follow-up (mean difference $-5.4,95 \% \mathrm{Cl}-19.1$ to 8.3). Secondary outcomes also showed no significant difference between intervention groups.

Conclusion: The effects of the Back on Track intervention are comparable to the effects of primary care physiotherapy as usual in patients with CLBP who experience low complex psychosocial complaints. Well-powered studies with sufficient methodological quality are needed to accurately detect the difference in effects between the interventions.

\section{KEYWORDS}

Low back pain, chronic pain, cognitive behavioural therapy, physical therapy, primary health care 


\section{INTRODUCTION}

Back pain, including neck pain, has the highest incidence rate of all health-related problems in the Netherlands. ' For low back pain (LBP) specifically, a lifetime prevalence of even up to $84 \%$ has been reported. ${ }^{2,3}$ Therefore, LBP is considered as a common health problem. LBP can be classified as acute ( $<6$ weeks of duration), subacute (6-12 weeks of duration), or chronic (CLBP, $\geq 12$ weeks of duration). Although physical or biomedical factors are suggested to trigger the onset of the LBP in the acute phase ${ }^{4}$, psychosocial factors have been found to influence the persistence of pain and disability (i.e. the development of CLBP). ${ }^{5}$

Due to the role of psychosocial factors in the persistence of LBP, therapy recommendations for patients with CLBP are directed towards a biopsychosocial approach. ${ }^{6}$ The beneficial effects of therapies with a biopsychosocial approach in multidisciplinary care settings have been confirmed by several systematic reviews. ${ }^{7-9}$ A multidisciplinary intervention is however not indicated and needed for every patient with CLBP. In the Netherlands for example, only patients who experience moderate to complex psychosocial complaints and who are, due to these complaints, at least moderately disabled at functional level, are referred to a multidisciplinary intervention (often provided in a secondary or tertiary care setting). ${ }^{10}$ Patients in which psychosocial complaints are present but influence daily life functioning to a lesser extent are referred to physiotherapy interventions in primary care. Physiotherapy interventions however include exercise therapy and manual therapy (i.e. manipulations and massage) ${ }^{11}$ and therefore not a specific biopsychosocial intervention.

To what extent a biopsychosocial approach would also be valuable in a primary care physiotherapy programme is inconclusive. To date, few studies investigated the differences in effects between a biopsychosocial primary care intervention and a (more biomedically oriented) physiotherapy programme, reporting mixed results. ${ }^{12}$ Some studies found significant beneficial effects in favour of the biopsychosocial primary care intervention (i.e. as compared to manual therapy or usual physiotherapy), ${ }^{13,14}$ while others reported no differences in effectiveness (as compared to motor control exercises and guideline physiotherapy). ${ }^{15,16}$ The varying findings could be a result of e.g. the selection procedure (patients not specifically selected on psychosocial complaints), the content of the intervention (different cognitive-behavioural elements used), physiotherapist's competence and adherence to the biopsychosocial protocol (including amount of cognitive-behavioural training and support). We noticed that a new study considering mentioned reasons was needed to study the effectiveness of a biopsychosocial primary care intervention. For this reason, a new biopsychosocial primary care intervention 'Back on Track' has been developed based on existing multidisciplinary biopsychosocial interventions (secondary care) but specifically adapted to match the patient's needs and the competence of trained physiotherapists in a primary care physiotherapy setting. ${ }^{17}$ The aim of this study was to compare the effects of the Back on Track intervention with primary care physiotherapy as usual (guideline based). The research question for this study was therefore: What is the difference in treatment effect (change in functional disability) between the new biopsychosocial primary care intervention 'Back on Track' and primary care physiotherapy as usual at post-treatment and 3 months post-treatment in a subgroup of patients with CLBP who experience psychosocial complaints that influences daily life functioning minimally? 


\section{METHOD}

\section{DESIGN}

This study is a pragmatic multicentre double-blind Randomised Controlled Trial (RCT). Ethical approval was provided by the Medical Ethics committee of the University Hospital of Maastricht and Maastricht University (METC azM/UM; METC143019). The study was monitored by the Clinical Trial Centre Maastricht (CTCM), the Netherlands. An extensive description of the study protocol has been published previously. ${ }^{18}$

The inclusion period lasted from August 2014-May 2016. Recruitment was conducted by physicians specialized in pain rehabilitation, working at the Maastricht University Medical Centre (MUMC+), the Netherlands. In case patients indicated to be interested in study participation, patients were invited for an intake at Maastricht University (UM) where they received additional information regarding the study. They provided written consent to participate and agreed that previously collected questionnaires in daily care were used for this study (i.e. questionnaires completed prior to the consultation with the physician). In addition, patients completed baseline questionnaires at the intake session. Patients were centrally randomised to either the Back on Track intervention or primary care physiotherapy as usual using block-randomisations via a computerized random number generator. The allocation sequence was accessible for the research assistant only and concealed for patients, physicians (recruiter), physiotherapists, researchers and data analysts. Researchers, data analysts, and patients remained blinded for treatment allocation. The research assistant, physiotherapists and physicians could not be blinded during treatment since they were involved in the intervention/logistics of the study.

\section{PARTICIPANTS, THERAPISTS, CENTRES}

Adult patients (18-65 years) were eligible for inclusion if they had CLBP for $\geq 12$ weeks, psychosocial were present but not complex, and functional disability was low to moderate. The classification of psychosocial complaints and level of disability was based on the expert opinion of the referring physicians in rehabilitation medicine. In addition, physicians had access to questionnaires completed by patients before the first consultation which could be used as support to the classification (i.e. Pain Catastrophizing Scale (PCS; range score $\geq 18-20$ ), Hospital Anxiety and Depression Scale (HADS; range score 8-11), RAND-36 item Health Survey (RAND-36; score > 42), Numeric Rating Scale (NRS; score > 4)). Patients needed to speak and read Dutch sufficiently, and to be willing to receive a biopsychosocial treatment approach. Patients were excluded in case of specific CLBP (e.g. infection, tumour, osteoporosis, fracture, structural deformation, inflammatory process, radicular syndrome or cauda equina syndrome), pregnancy, and/or suspicion of an (underlying) psychiatric disease.

Physiotherapy treatments were provided by physiotherapists working in primary care practices located in Maastricht, the Netherlands and the surrounding area. Four practices provided the Back on Track intervention, four practices primary care physiotherapy as usual. Practices were 
selected on appropriate facilities, willingness and motivation to participate. No specific criteria for physiotherapists were formulated, except that physiotherapists providing the Back on Track intervention should generally be motivated to provide a protocolled biopsychosocial oriented intervention and to receive a training programme.

\section{INTERVENTIONS}

All patients received medical education from the referring physician prior to recruitment. This included information about pain physiology and the difference between acute and chronic pain. Physicians reassured that patients could improve the level of daily activities despite pain and explained that hurt would not equal harm.

Patients randomised to the Back on Track intervention received four individual sessions (30 minutes) and eight group sessions (60 minutes). The Back on Track intervention stimulated patients to gain insight in pain mechanisms, behaviour and beliefs, coping styles, goal-setting and self-management strategies. The intervention included elements of cognitive behavioural approaches such as Graded Activity and Exposure in vivo. Patients furthermore received a workbook with basic information about the treatment and homework assignments (described in detail elsewhere. ${ }^{17}$ Compliance threshold with the Back on Track intervention was reached if a patient attended at least three individual sessions and at least half of the group sessions. Physiotherapists received a manual with standardized treatment sessions prior to the start. Physiotherapists were trained to deliver the Back on Track intervention according to the protocol and to deliver cognitive behavioural elements (3 meetings of 4 hours). Two booster sessions were offered to physiotherapists during the study.

Patients randomised to primary care as usual received individual physiotherapy sessions according to physiotherapists' best practice and the Dutch profession-specific guideline for LBP..$^{19}$ The number of sessions was at the discretion of the physiotherapists, but to a maximum of twelve sessions to keep the length comparable to the Back on Track intervention. A log was kept regarding the content, frequency and duration of the therapy. Physiotherapists received no treatment manual or training for treatment delivery. Physiotherapists only received one meeting to be informed about the study logistics. They were not informed about the content of the Back on Track intervention to prevent cross-contamination.

\section{OUTCOME MEASURES}

Patients completed questionnaires at baseline, directly post-treatment and at 3 months post-treatment. The primary outcome was functional disability using the Quebec Back Pain Disability Scale (QBPDS, 0-100). ${ }^{20-22}$ Secondary outcomes were: (1) pain intensity, measured with the Numeric Rating Scale (NRS) ranging from 0 (no pain) to 10 (worst pain); (2) pain catastrophizing, measured with the PCS (0-52):23,24 (3) anxiety and depression, measured with the HADS (0-21 per subscale); ${ }^{25}$ (4) pain-related fear, measured with the Tampa Scale of Kinesiophobia (TSK, 17-68);26,27 (5) Self-efficacy, measured with 10-itemed Pain Self Efficacy Questionnaire (PSEQ, 0-60); ${ }^{28}$ and (6) global perceived effect (GPE), measured with a 7-point Likert scale ranging from "completely 
recovered" to "worse than ever", and "satisfied" to "very dissatisfied".29 Patients furthermore completed the Credibility and Expectancy Questionnaire (CEQ) ${ }^{30,31}$ directly after the first individual session to investigate the credibility and expectancy related to the treatment (credibility score 5-45, expectancy score 6-54). Directly after having received the entire intervention, patients were asked which intervention they thought to have received.

\section{DATA ANALYSIS}

Baseline scores of Smeets et al. ${ }^{32}$ were used to calculate the sample size for this study. We aimed to detect a between-group difference of at least 15\% on the QBPDS, which equals to 7 points in the study of Smeets et al. A 2-tailed test with a significance level of 0.05 , a power of $80 \%$, and a drop-out rate of $20 \%$ was used. In order to take the longitudinal design of the study into account, sample size calculation was adjusted for repeated measures. Sample size calculation resulted in 43 patients per group.

Descriptive statistics were used to summarize baseline characteristics of patients. Frequencies are presented for categorical variables, means and standard deviations (SDs) for normally distributed continuous variables, and medians and ranges for not normally distributed continuous data (i.e. baseline pain, anxiety and physical functioning). Baseline similarity was determined using chi-square tests for categorical variables, and independent t-tests or Mann-Whitney $U$ (non-parametric) tests for continuous variables.

Linear mixed model analysis was used to determine between and within group differences over time, using the $3 \times 3$ identity covariance structure. No imputation strategy was used since mixed model analysis allows for missing data. Patient was included as random factor. For each patient, there are three measurements: pre, post and 3 months after end of treatment. Treatment, time (three categories), and time $\times$ treatment were included as fixed factors. Baseline age, duration of LBP and sex were also considered in the model, but apart from duration of LBP in modelling HADS subscale depression and in modelling TSK, none of these were significant and therefore not included in the final model. The influence of credibility and expectancy on QBPDS outcome was determined by adding credibility and expectancy separately to the model. An interaction term (credibility $\times$ treatment or expectancy $\times$ treatment) was subsequently added to determine differences between treatment groups. Analysis was by intention-to-treat. Significance level was set at 0.05 .

\section{RESULTS}

\section{FLOW OF PATIENTS, THERAPISTS, AND CENTRES}

Thirty-one eligible patients were referred to the Back on Track study (Figure 1). Twenty-five patients gave written consent and were randomised. Table 1 provides baseline characteristics of randomised patients per intervention. The mean age of participating patients was 44 years (SD 12.2, range 18-62). Fifty-six percent was female (14 of 25). The level of psychosocial complaints was at group low as patients experienced a mean level of catastrophizing of 15 (SD 9.6), a median 
level of anxiety of 4 (range 0-18), a mean level of depression of 3.9 (SD 3.0), a mean level of kinesiophobia of 33 (SD 6.6), and a median level of physical functioning of 70 (range 20-95). There were no significant differences at baseline between the two groups, as well as between patients who discontinued or continued the intervention (Table 1).

The Back on Track intervention was provided by four physiotherapists. Professional experience of physiotherapists ranged from no experience (just graduated) to 31 years of experience as physiotherapist. All four physiotherapists attended the three training sessions prior to the study.

Figure 1 Flow chart of the study participants

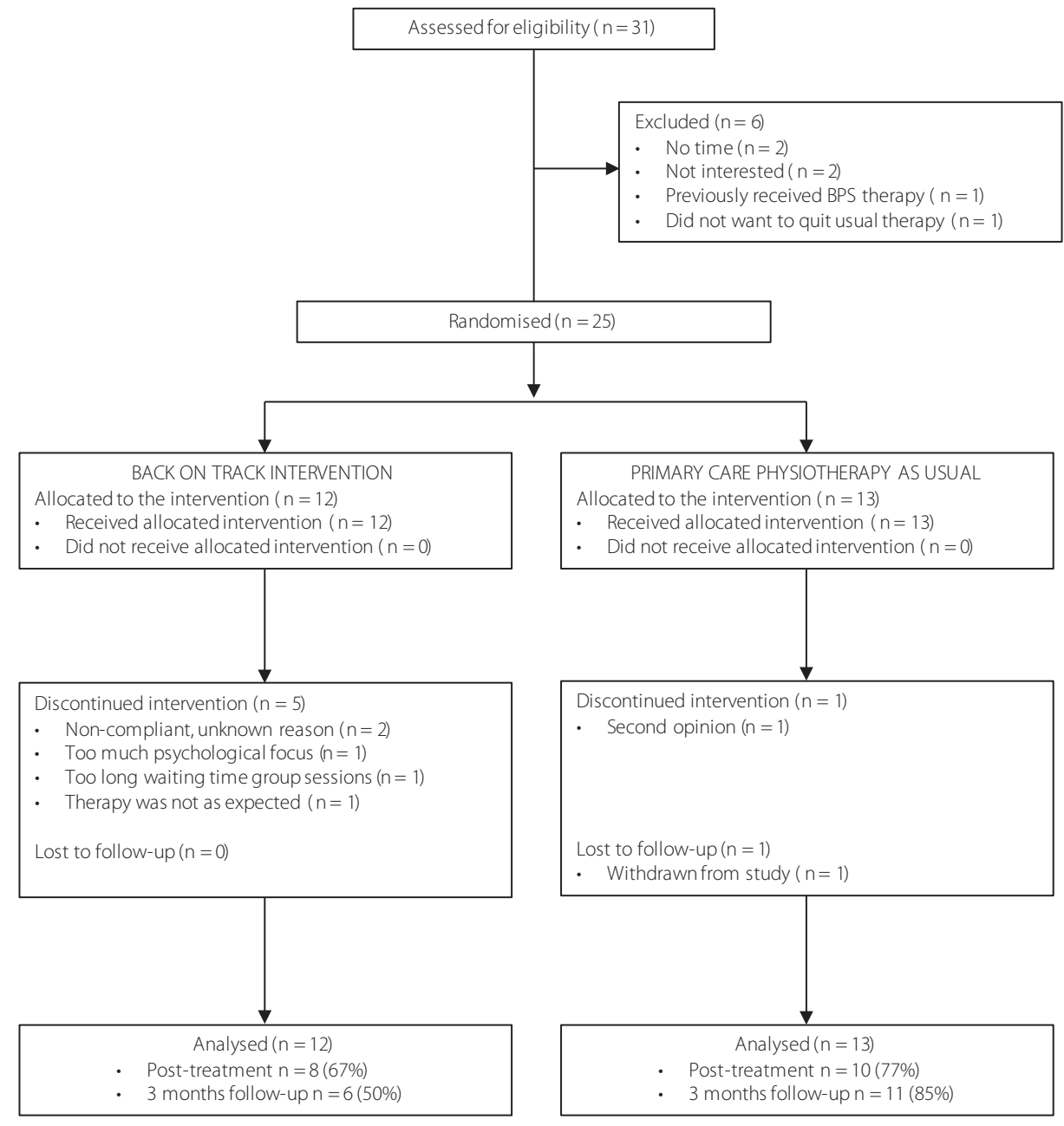


Table 1 Baseline characteristics of study participants

\begin{tabular}{|c|c|c|}
\hline & $\begin{array}{l}\text { Back on Track } \\
\text { Intervention } \\
(\mathrm{n}=12)\end{array}$ & $\begin{array}{c}\text { Physiotherapy } \\
\text { as usual } \\
(\mathrm{n}=13)\end{array}$ \\
\hline Age (years) - mean (SD) & $43(12)$ & $45(12)$ \\
\hline Sex (female) - n (\%) & $7(58 \%)$ & $7(54 \%)$ \\
\hline \multicolumn{3}{|l|}{ Nationality - n (\%) } \\
\hline Dutch & $12(100 \%)$ & $12(92 \%)$ \\
\hline Other & $0(0 \%)$ & $1(8 \%)$ \\
\hline \multicolumn{3}{|l|}{ Employment - n (\%) } \\
\hline Full-time & $6(50 \%)$ & $6(46 \%)$ \\
\hline Part-time & $5(42 \%)$ & $6(46 \%)$ \\
\hline Full-time education & $1(8 \%)$ & $1(8 \%)$ \\
\hline \multicolumn{3}{|l|}{ Education level - n (\%) } \\
\hline Low & $2(17 \%)$ & $3(23 \%)$ \\
\hline Middle & $5(42 \%)$ & $8(62 \%)$ \\
\hline High & $5(42 \%)$ & $2(15 \%)$ \\
\hline \multicolumn{3}{|l|}{ Duration of low back pain (since first onset) - $\mathrm{n}(\%)$} \\
\hline $3-6$ months & $1(8 \%)$ & $0(0 \%)$ \\
\hline $6-12$ months & $1(8 \%)$ & $1(8 \%)$ \\
\hline $1-2$ years & $1(8 \%)$ & $3(23 \%)$ \\
\hline $2-5$ years & $5(42 \%)$ & $7(54 \%)$ \\
\hline$>5$ years & $4(33 \%)$ & $2(15 \%)$ \\
\hline Functional disability (QBPDS) - mean (SD) & $31(12)$ & $38(15)$ \\
\hline Functional disability (PDI) - mean (SD) & $25(15.4)$ & $28(12.3)$ \\
\hline Pain intensity (NRS) - median (range) & $6.0(2-8)$ & $7.0(3-9)$ \\
\hline Pain catastrophizing (PCS) - mean (SD) & $14(10.4)$ & $16(9.2)$ \\
\hline \multicolumn{3}{|l|}{ Anxiety and Depression (HADS) } \\
\hline Anxiety - median (range) & $5.0(0-9)$ & $4.0(0-18)$ \\
\hline Depression - mean (SD) & $3.8(3.2)$ & $3.9(3.0)$ \\
\hline Kinesiophobia (TSK) - mean (SD) & $32(7.2)$ & $35(6.1)$ \\
\hline Pain self-efficacy (PSEQ) - mean (SD) & $46(8.8)$ & $42(12.9)$ \\
\hline Physical functioning (RAND-36, subscale) - median (range) & $68(25-95)$ & $70(20-75)$ \\
\hline Mental health (RAND-36, subscale) - mean (SD) & $78(16.8)$ & $75(21.6)$ \\
\hline Credibility $(C E Q)^{*}-$ mean (SD) & $30(8.7)$ & $32(6.2)$ \\
\hline Expectancy (CEQ)* - mean (SD) & $36(11)$ & $39(8.0)$ \\
\hline
\end{tabular}

QBPDS = Quebec Back Pain Disability Scale (0-100); NRS = Numeric Rating Scale (0-10), average pain last week; PCS = Pain Catastrophizing Scale (0-52); HADS = Hospital Anxiety and Depression Scale (0-21 per subscale), PSEQ = Pain Self Efficacy Questionnaire (0-60); TSK = Tampa Scale for Kinesiophobia (17-68); RAND-36 = RAND-36 item Health Survey (0-100 per subscale); Credibility and Expectancy Scale (credibility 5-45, expectancy 6-54).

${ }^{*}$ CEQ is measured directly after the first therapy session; Back on Track $n=8$, physiotherapy as usual $n=11$ 


\section{ADHERENCE TO THE STUDY PROTOCOL}

The average number of therapy sessions attended by patients in the Back on Track intervention was 7.9 (range 2-12). Over half of the patients met the compliance threshold (58\%). Only patients who discontinued the intervention did not meet the compliance threshold (reasons are listed in Figure 1). Of the patients who did continue the intervention, three patients missed one session only. Reasons for non-attendance were: forgot to join the therapy session ( $n=1)$, and unknown reasons $(n=2)$.

The average number of therapy sessions in the physiotherapy as usual intervention was 8.2 (range 3-12). The content of the physiotherapy as usual sessions mainly comprised of mobilisation techniques, core stability training, specific lower back exercises, massages and manual therapy. Directly after the intervention, one of the 19 patients who responded to the question to which intervention he or she was allocated to, did not know the allocation (Back on Track). Five patients indicated the allocation wrong and 13 patients the right intervention.

\section{EFFECTS OF THE INTERVENTIONS}

Mixed model analyses showed no significant differences in functional disability between the Back on Track intervention and primary care physiotherapy as usual directly after treatment and at 3 months follow-up (Table 2). Mixed model analyses furthermore showed no significant between-group difference on other secondary outcomes at both time points. In addition, higher scores on credibility and expectancy did not significantly result in improvements in functional disability. Also its influence did not significantly differ between the intervention groups ( $p>0.05$ ). GPE scores at both time points also did not differ significantly between interventions ( $p>0.05$ ).

During the study, no serious adverse events were reported. One adverse event was reported in the physiotherapy as usual group, but this was not related to the therapy (i.e. LBP due to fall from stairs at home). 
Table 2 Estimates of clinical effectiveness at post-treatment and 3 months follow-up

\begin{tabular}{|c|c|c|c|c|c|c|}
\hline & \multicolumn{2}{|c|}{$\begin{array}{l}\text { Mean score }(95 \% \mathrm{Cl}) \text { within } \\
\text { intervention group }\end{array}$} & \multicolumn{2}{|c|}{$\begin{array}{l}\text { Mean change }(95 \% \mathrm{Cl}) \text { within } \\
\text { intervention group }\end{array}$} & \multirow{2}{*}{$\begin{array}{l}\text { Mean treatment } \\
\text { difference }(95 \% \\
\text { CI) between } \\
\text { intervention } \\
\text { groups }\end{array}$} & \multirow{2}{*}{ p-value } \\
\hline & $\begin{array}{l}\text { Back on Track } \\
\text { Intervention } \\
\quad(\mathrm{n}=12)\end{array}$ & $\begin{array}{c}\text { Physiotherapy } \\
\text { as usual } \\
(\mathrm{n}=13)\end{array}$ & $\begin{array}{c}\text { Back on Track } \\
\text { intervention } \\
\quad(n=12)\end{array}$ & $\begin{array}{l}\text { Physiotherapy } \\
\text { as usual } \\
(\mathrm{n}=13)\end{array}$ & & \\
\hline \multicolumn{7}{|l|}{ QBPDS } \\
\hline Baseline & 30.7 (20.5 to 40.8$)$ & 37.8 (28.1 to 47.6$)$ & & & & \\
\hline Post-treatment & 27.2 (15.8 to 38.5$)$ & 34.2 (23.8 to 44.6$)$ & $-3.5(-13.2$ to 6.2$)$ & $-3.6(-12.3$ to 5.1$)$ & $0.10(-12.9$ to 13.1$)$ & .987 \\
\hline 3 months follow-up & $21.9(9.6$ to 34.1$)$ & 34.5 (24.3 to 44.6$)$ & $-8.8(-19.6$ to 2.0$)$ & $-3.4(-11.8$ to 5.0$)$ & $-5.4(-19.1$ to 8.3$)$ & .428 \\
\hline \multicolumn{7}{|l|}{ NRS } \\
\hline Baseline & 5.3 (4.0 to 6.5$)$ & 6.5 (5.3 to 7.8$)$ & & & & \\
\hline Post-treatment & 5.5 (3.9 to 7.0$)$ & $5.7(4.3$ to 7.1$)$ & $0.22(-1.5$ to 2.0$)$ & $-0.86(-2.4$ to 0.72$)$ & $1.1(-1.3$ to 3.4$)$ & .356 \\
\hline 3 months follow-up & $5.0(3.2$ to 6.7$)$ & $5.6(4.3$ to 7.0$)$ & $-0.28(-2.2$ to 1.6$)$ & $-0.90(-2.4$ to 0.63$)$ & $0.63(-1.8$ to 3.1$)$ & .609 \\
\hline \multicolumn{7}{|l|}{ PCS } \\
\hline Baseline & 14.3 (8.7 to 19.8$)$ & $15.8(10.4$ to 21.1$)$ & & & & \\
\hline Post-treatment & $8.4(2.0$ to 14.8$)$ & 16.2 (10.4 to 22.0$)$ & $-5.8(-11.8$ to 0.14$)$ & $0.41(-5.0$ to 5.8$)$ & $-6.2(-14.3$ to 1.8$)$ & .123 \\
\hline 3 months follow-up & 12.6 (5.6 to 19.6$)$ & 15.7 (10.1 to 21.4$)$ & $-1.7(-8.3$ to 5.0$)$ & $-0.05(-5.3$ to 5.2$)$ & $-1.6(-10.1$ to 6.8$)$ & .697 \\
\hline \multicolumn{7}{|l|}{ HADS, subscale anxiety } \\
\hline Baseline & $4.2(2.1$ to 6.2$)$ & 5.3 (3.3 to 7.3$)$ & & & & \\
\hline Post-treatment & $3.6(1.3$ to 5.9$)$ & $4.3(2.3$ to 6.4$)$ & $-0.57(-2.3$ to 1.2$)$ & $-0.96(-2.4$ to 0.47$)$ & $0.39(-1.9$ to 2.7$)$ & .728 \\
\hline 3 months follow-up & $3.3(0.90$ to 5.7$)$ & 4.7 (2.7 to 6.8$)$ & $-0.88(-2.8$ to 0.99$)$ & $-0.60(-2.0$ to 0.84$)$ & $-0.28(-2.6$ to 2.1$)$ & .808 \\
\hline \multicolumn{7}{|c|}{ HADS, subscale depression* } \\
\hline Baseline & $5.0(3.0$ to 6.9$)$ & 4.8 (2.7 to 6.8$)$ & & & & \\
\hline Post-treatment & $4.0(1.8$ to 6.2$)$ & $4.8(2.6$ to 6.9$)$ & $-0.91(-2.9$ to 1.1$)$ & $0.03(-1.6$ to 1.7$)$ & $-0.94(-3.5$ to 1.6$)$ & .459 \\
\hline 3 months follow-up & $3.1(0.87$ to 5.4$)$ & $5.3(3.2$ to 7.5$)$ & $-1.8(-3.9$ to 0.28$)$ & $0.57(-1.1$ to 2.2$)$ & $-2.4(-5.0$ to 0.26$)$ & .076 \\
\hline \multicolumn{7}{|l|}{ PSEQ } \\
\hline Baseline & 45.9 (39.1 to 52.8$)$ & 42.3 (35.7 to 48.9$)$ & & & & \\
\hline Post-treatment & 45.8 (37.9 to 53.6$)$ & 41.9 (34.8 to 49.1$)$ & $-0.14(-7.7$ to 7.4$)$ & $-0.38(-7.2$ to 6.4$)$ & $0.23(-9.9$ to 10.4$)$ & .962 \\
\hline 3 months follow-up & 50.4 (41.7 to 59.0) & 42.8 (35.8 to 49.7$)$ & $4.5(-3.9$ to 12.9$)$ & $0.44(-6.1$ to 7.0$)$ & $4.1(-6.6$ to 14.7$)$ & .445 \\
\hline \multicolumn{7}{|l|}{$\mathrm{TSK}^{*}$} \\
\hline Baseline & 34.7 (30.6 to 38.7) & $36.0(31.7$ to 40.4$)$ & & & & \\
\hline Post-treatment & 34.0 (29.5 to 38.6$)$ & 40.1 (35.5 to 44.7$)$ & $-0.64(-5.1$ to 3.8$)$ & $4.1(0.04$ to 8.1$)$ & $-4.7(-10.7$ to 1.3$)$ & .117 \\
\hline 3 months follow-up & 34.5 (29.5 to 39.4$)$ & 38.4 (33.8 to 42.9$)$ & $-0.21(-5.2$ to 4.7$)$ & $2.3(-1.6$ to 6.2$)$ & $-2.5(-8.8$ to 3.8$)$ & .420 \\
\hline \multicolumn{7}{|l|}{ GPE, subscale recovery ${ }^{\#}$} \\
\hline Post-treatment & $4.8(4.2$ to 5.3$)$ & $4.5(4.0$ to 5.0$)$ & & & & \\
\hline 3 months follow-up & $4.9(4.3$ to 5.6$)$ & 4.5 (4.0 to 5.0$)$ & & & & \\
\hline \multicolumn{7}{|l|}{ GPE, subscale satisfaction" } \\
\hline Post-treatment & 4.6 (3.4 to 5.8$)$ & 4.5 (3.4 to 5.5$)$ & & & & \\
\hline 3 months follow-up & $4.6(3.3$ to 5.9$)$ & $3.7(2.7$ to 4.7$)$ & & & & \\
\hline
\end{tabular}

QBPDS = Quebec Back Pain Disability Scale (0-100); NRS = Numeric Rating Scale (0-10), average pain last week; PCS = Pain Catastrophizing Scale (0-52); HADS = Hospital Anxiety and Depression Scale (0-21 per subscale), PSEQ = Pain Self Efficacy Questionnaire (0-60); TSK = Tampa Scale for Kinesiophobia (17-68), GPE = Global Perceived Effect (1-7 per subscale).

* Mean score and mean change were as average over the five categories of the significant variable duration of low back pain.

" Back on Track $n=8$, physiotherapy as usual $n=11$ 


\section{DISCUSSION}

This RCT investigated the difference in effectiveness between a biopsychosocial primary care intervention and primary care physiotherapy as usual in a subgroup of patients with CLBP. Patients included in this study experienced psychosocial complaints of low complexity which influence daily life functioning minimally. In the Netherlands, this subgroup of patients is usually referred to primary care physiotherapy treatments. The improvements in functional disability, as well as in other secondary outcomes, were not significantly different between the two interventions at post-treatment and 3 months follow-up. Based on these findings it can be suggested that no therapy is better than the other in patients with CLBP in which psychosocial factors are only of minimal influence.

Findings of this study are in line with findings of van der Roer et al. ${ }^{16}$ and Macedo et al. ${ }^{15}$ who both compared a Graded Activity intervention with another, more biomedically oriented, physiotherapy programme (guideline physiotherapy as usual and motor control exercises, respectively). These studies also found no differences in improvements in the level of functional disability between the interventions at short, medium and long term. Although the Back on Track intervention design differed from the Graded Activity interventions as it also included other cognitive-behavioural elements (e.g. exposure in vivo), these studies confirm the assumption that the type of therapy makes little difference for patients referred to a physiotherapy treatment in primary care.

Of notice is that our study included only 25 patients in total, while it was powered at 86 patients. The lack of power makes interpretation of our results difficult and limits the comparison with other studies. It is possible that there were differences in effects, but the study was unable to detect them. Drawing conclusions and a direct comparison with mentioned studies should therefore be made with caution.

The inability to achieve the desired sample size was caused by the recruitment approach used. We considered recruitment via physicians important since physicians were specialised in chronic pain rehabilitation treatments, experienced in identifying psychosocial complaints and experienced in delivering pain education. We expected this would lead to the referral of a homogeneous group of patients, well-prepared for a biopsychosocial approach. In the time of receiving medical ethical approval for the study, Dutch legislation changed. Secondary care for patients with non-complex psychosocial complaints was discouraged and fewer patients were referred to physicians. Despite extra efforts to stimulate recruitment (i.e. by contacting general practitioners (GPs), physiotherapists and other health care specialists), the desired number of patients could by far not be achieved.

Another limitation was that more patients discontinued the Back on Track intervention than physiotherapy as usual. One reason was that the waiting time for group sessions was too long. The study had difficulties with generating groups, caused by the slow study recruitment. Patients therefore had to wait for group sessions to start. This was not an issue in the physiotherapy as usual intervention as it comprised of individual sessions only. An additional reason was that patients had different expectations about the Back on Track intervention. Physicians reported in focus group interview that, due to the allocation concealment, they could not properly prepare 
patients for a biopsychosocial intervention as they would usually do. ${ }^{33}$ So, the study procedures seem to have restricted physician's usual care. To what extent this has influenced study outcomes is unknown.

A priori, we published our study protocol and considered this, in combination with the study design (a double-blinded RCT), a strength of the study. ${ }^{18}$ Randomisation seemed successful since baseline characteristics were equally distributed over interventions. We expected that patient blinding would be affected as patients might recognise the allocated therapy. Six patients either did not know or thought they were allocated to the other therapy. This indicates that blinding was maintained to some extent. An additional strength was that physiotherapists who provided the Back on Track intervention were satisfied with the training and could sufficiently deliver cognitive behavioural protocol elements. ${ }^{33}$ It is therefore assumed that the Back on Track intervention was provided as intended and delivery did not influence therapy outcomes.

For future studies, it can be recommended to reconsider the recruitment strategy, the biopsychosocial intervention design, and if desirable, the study design. Alternative recruitment strategies are via GPs or primary care physiotherapist. These are more frequently consulted by patients with low complex psychosocial complaints than physicians in secondary care nowadays. Additional screening tools might be helpful to ensure correct identification of patient's psychosocial complaints ${ }^{34,35}$ as previous studies have shown that GPs and physiotherapists seem not or only partially able to correctly identify psychosocial complaints. ${ }^{36,37}$ As soon as the patient is allocated to the biopsychosocial intervention, the patient should be well-prepared for a biopsychosocial approach. Being well-prepared and satisfied with the therapy likely improves therapy compliance. Compliance in turn has been shown to optimise therapy effects. ${ }^{38}$ As therapy expectations did not always match and compliance was not optimal in our study, devoting an additional session to provide pain education and to discuss patient's expectations might be required in future. Furthermore, group therapy should be considered only if recruitment or referral is sufficient to generated groups in time. An alternative study design could furthermore be recommended such as a single-case design in which a patient acts as its own control. This design requires less patients, although more frequent measurements and different logistics need to be performed. ${ }^{39}$ Overall, we expect that the discussed findings and recommendations will likely be helpful for developing future feasible, high-quality studies, which remain required to determine the difference in effectiveness between a biopsychosocial intervention in a primary care physiotherapy setting and primary care as usual for patients with CLBP.

\section{ACKNOWLEDGEMENTS}

We acknowledge Marion de Mooij for her assistance during the study; the Department of Rehabilitation in medicine MUMC + and physicians for the recruitment of patients; dr. Paul Willems and members of the Spine Centre MUMC + and department of Anaesthesiology for referring patients; Fy'net Collaboration, dr. Frans Abbink (Fysiotherapiepraktijk Abbink), Tom Hameleers (ICL Fysio), Remco Reijnders \& Germaine Neumann (Fysiohof), Yvonne Janss (Fysiotherapiepraktijk Yvonne Janss), Rick Kessels (Fysio Zuyd Caberg), Judith Giessen-Ploemen (Fysiotherapiepraktijk Giessen-Ploemen) for providing treatments to patients in the study. 


\section{REFERENCES}

1. RIVM. Ranglijst ziekten op basis van incidentie. 2011; https://www.volksgezondheidenzorg.info/ranglijst/ranglijst-ziekten-op-basis-van-incidentie. Accessed 24072017.

2. Airaksinen O, Brox Jl, Cedraschi C, et al. Chapter 4. European guidelines for the management of chronic nonspecific low back pain. European spine journal : official publication of the European Spine Society, the European Spinal Deformity Society, and the European Section of the Cervical Spine Research Society. 2006;15 Suppl 2:S192-300.

3. Balague F, Mannion AF, Pellise F, Cedraschi C. Non-specific low back pain. Lancet. 2012;379(9814):482491.

4. Stevens ML, Steffens D, Ferreira ML, et al. Patients' and Physiotherapists' Views on Triggers for Low Back Pain. Spine. 2016;41(4):E218-224.

5. Chou R, Shekelle P.Will this patient develop persistent disabling low back pain? JAMA. 2010;303(13):12951302.

6. Koes BW, van Tulder M, Lin CW, Macedo LG, McAuley J, Maher C. An updated overview of clinical guidelines for the management of non-specific low back pain in primary care. European spine journal : official publication of the European Spine Society, the European Spinal Deformity Society, and the European Section of the Cervical Spine Research Society. 2010;19(12):2075-2094.

7. Kamper SJ, Apeldoorn AT, Chiarotto A, et al. Multidisciplinary biopsychosocial rehabilitation for chronic low back pain. The Cochrane database of systematic reviews. 2014;9:CD000963.

8. Henschke N, Ostelo RW, van Tulder MW, et al. Behavioural treatment for chronic low-back pain. The Cochrane database of systematic reviews. 2010(7):CD002014.

9. Hall A, Richmond H, Copsey B, et al. Physiotherapist-delivered cognitive-behavioural interventions are effective for low back pain, but can they be replicated in clinical practice? A systematic review. Disability and rehabilitation. 2016:1-9.

10. Boonstra AM, Bühring M, Brouwers M, Bosma F, Schiphorst Preuper R. Patiënten met chronische pijnklachten op het grensvlak van revalidatiegeneeskunde en psychiatrie. Ned Tijdschr Pijn bestr. 2008;27(34):5-9.

11. Swinkels IC, van den Ende CH, van den Bosch W, Dekker J, Wimmers RH. Physiotherapy management of low back pain: does practice match the Dutch guidelines? The Australian journal of physiotherapy. 2005;51(1):35-41.

12. Van Erp RMA, Huijnen IPJ, Jakobs MLG, Kleijnen J, Smeets RJEM. Effectiveness of primary care interventions using a biopsychosocial approach in chronic low back pain: a systematic review. Submitted.

13. Walti P, Kool J, Luomajoki H. Short-term effect on pain and function of neurophysiological education and sensorimotor retraining compared to usual physiotherapy in patients with chronic or recurrent non-specific low back pain, a pilot randomized controlled trial. BMC musculoskeletal disorders. 2015;16:83.

14. Vibe Fersum K, O'Sullivan P, Skouen JS, Smith A, Kvale A. Efficacy of classification-based cognitive functional therapy in patients with non-specific chronic low back pain: a randomized controlled trial. European journal of pain. 2013;17(6):916-928.

15. Macedo LG, Latimer J, Maher CG, et al. Effect of motor control exercises versus graded activity in patients with chronic nonspecific low back pain: a randomized controlled trial. Physical therapy. 2012;92(3):363377. 
16. van der Roer N, van Tulder M, Barendse J, Knol D, van Mechelen W, de Vet H. Intensive group training protocol versus guideline physiotherapy for patients with chronic low back pain: a randomised controlled trial. European spine journal : official publication of the European Spine Society, the European Spinal Deformity Society, and the European Section of the Cervical Spine Research Society. 2008;17(9):1193-1200.

17. van Erp RMA, Huijnen IPJ, Koke AJA, Abbink FE, den Hollander M, Smeets R. Development and content of the biopsychosocial primary care intervention 'Back on Track' for a subgroup of people with chronic low back pain. Physiotherapy. 2017;103(2):160-166.

18. van Erp RM, Huijnen IP, Verbunt JA, Smeets RJ. A biopsychosocial primary care intervention (Back on Track) versus primary care as usual in a subgroup of people with chronic low back pain: protocol for a randomised, controlled trial. J Physiother. 2015;61(3):155.

19. Staal JB, M. HEJ, Heijmans M, et al. KNGF-richtlijn Lage-rugpijn. Amersfoort: Drukkerij De Gans;2013.

20. Kopec JA, Esdaile JM, Abrahamowicz M, et al. The Quebec Back Pain Disability Scale. Measurement properties. Spine. 1995;20(3):341-352.

21. Schoppink LE, van Tulder MW, Koes BW, Beurskens SA, de Bie RA. Reliability and validity of the Dutch adaptation of the Quebec Back Pain Disability Scale. Physical therapy. 1996;76(3):268-275.

22. Smeets R, Koke A, Lin CW, Ferreira M, Demoulin C. Measures of function in low back pain/disorders: Low Back Pain Rating Scale (LBPRS), Oswestry Disability Index (ODI), Progressive Isoinertial Lifting Evaluation (PILE), Quebec Back Pain Disability Scale (QBPDS), and Roland-Morris Disability Questionnaire (RDQ). Arthritis care \& research. 2011;63 Suppl 11:S158-173.

23. Sullivan MJL, Bishop SR. The Pain Catastrophizing Scale: Development and Validation. Psychological Assessment. 1995;7:524-532.

24. Van Damme S, Crombez G, Vlaeyen J, Goubert L, Van den Broek A, Van Houdenhove B. De Pain Catastrophizing Scale: Psychometrische karakteristieken en normering. Gedragstherapie. 2000;33:209-220.

25. Zigmond AS, Snaith RP. The hospital anxiety and depression scale. Acta Psychiatr Scand. 1983;67(6):361-370.

26. Vlaeyen JW, Kole-Snijders AM, Boeren RG, van Eek H. Fear of movement/(re)injury in chronic low back pain and its relation to behavioral performance. Pain. 1995;62(3):363-372.

27. Goubert L, Crombez G, Vlaeyen J, van Damme S, van den Broeck A, van Houdenhove B. De Tampa Schaal voor Kinesiofobie: Psychometrische karakteristieken en normering (The Tampa scale Kinesiophobia: psychometric properties and norms). Gedrag Gezond. 2000;28:54-62.

28. Nicholas MK. The pain self-efficacy questionnaire: Taking pain into account. European journal of pain. 2007;11(2):153-163.

29. Kamper SJ, Ostelo RW, Knol DL, Maher CG, de Vet HC, Hancock MJ. Global Perceived Effect scales provided reliable assessments of health transition in people with musculoskeletal disorders, but ratings are strongly influenced by current status. Journal of clinical epidemiology. 2010;63(7):760766 e761.

30. Mertens VC, Moser A, Verbunt J, Smeets R, Goossens M. Content Validity of the Credibility and Expectancy Questionnaire in a Pain Rehabilitation Setting. Pain practice : the official journal of World Institute of Pain. 2016. 
31. Smeets RJ, Beelen S, Goossens ME, Schouten EG, Knottnerus JA, Vlaeyen JW. Treatment expectancy and credibility are associated with the outcome of both physical and cognitive-behavioral treatment in chronic low back pain. The Clinical journal of pain. 2008;24(4):305-315.

32. Smeets RJ, Vlaeyen JW, Hidding A, et al. Active rehabilitation for chronic low back pain: cognitive-behavioral, physical, or both? First direct post-treatment results from a randomized controlled trial [ISRCTN22714229]. BMC musculoskeletal disorders. 2006;7:5.

33. Van Erp RMA, Huijnen IPJ, Köke AJA, Verbunt JA, Smeets RJEM. Feasibility of the biopsychosocial primary care intervention 'Back on Track' for patients with chronic low back pain: a process and effect-evaluation. Submitted.

34. Hill JC, Dunn KM, Lewis M, et al. A primary care back pain screening tool: identifying patient subgroups for initial treatment. Arthritis and rheumatism. 2008;59(5):632-641.

35. Linton SJ, Nicholas M, MacDonald S. Development of a short form of the Orebro Musculoskeletal Pain Screening Questionnaire. Spine. 2011;36(22):1891-1895.

36. Jellema P, van der Windt DA, van der Horst HE, Blankenstein AH, Bouter LM, Stalman WA. Why is a treatment aimed at psychosocial factors not effective in patients with (sub)acute low back pain? Pain. 2005;118(3):350-359.

37. Synnott A, O'Keeffe M, Bunzli S, Dankaerts W, O'Sullivan P, O'Sullivan K. Physiotherapists may stigmatise or feel unprepared to treat people with low back pain and psychosocial factors that influence recovery: a systematic review. J Physiother. 2015;61(2):68-76.

38. Knox CR, Lall R, Hansen Z, Lamb SE. Treatment compliance and effectiveness of a cognitive behavioural intervention for low back pain: a complier average causal effect approach to the BeST data set. BMC musculoskeletal disorders. 2014;15:17.

39. Onghena P, Edgington ES. Customization of pain treatments: single-case design and analysis. The Clinical journal of pain. 2005;21(1):56-68; discussion 69-72. 


\title{
Chapter 6
}

\author{
Feasibility of the biopsychosocial \\ primary care intervention 'Back on Track' \\ for patients with chronic low back \\ pain: a process and effect-evaluation
}

Reni van Erp Ivan Huijnen Albère Köke Jeanine Verbunt Rob Smeets 



\section{ABSTRACT}

Purpose: To investigate the feasibility and effectiveness of a biopsychosocial integrated care programme (Back on Track) in patients with chronic low back pain (CLBP) with moderate complex psychosocial complaints.

Materials and methods: Mixed-method design. Physicians in rehabilitation medicine referred patients (age 18-65 yrs., low back pain $\geq 12$ wks., moderate complex psychosocial complaints) to the intervention (4 individual sessions, 8 group sessions) provided by trained physiotherapists in primary care. Physicians provided one consultation afterwards. Data from patients ( $n=18)$, physicians $(n=4)$ and physiotherapists $(n=12)$ were used for process and effect evaluation (attendance lists, questionnaires, audio recordings of therapy, focus groups).

Results: Physiotherapists were satisfied with the preceding training. Patients had significantly higher expectations than physiotherapists that the intervention would reduce pain intensity. Attendance was good for individual sessions, less for groups. Physiotherapists sufficiently delivered the intervention, although recruitment and contextual factors (physicians' approach to classify level of psychosocial complaints, communication, planning) influenced delivery and satisfaction. Patients significantly reduced functional disability (Quebec Back Pain Disability Scale) post treatment $(-8.3,95 \% \mathrm{Cl}-13.3$ to -2.7$)$ and at 3 months follow-up $(-7.6,95 \%$ $\mathrm{Cl}-12.9$ to -2.2$)$.

Conclusions: This study shows that a biopsychosocial integrated intervention is feasible and potentially effective in patients with CLBP.

\section{KEYWORDS}

Chronic low back pain, process evaluation, treatment fidelity, cognitive behavioural therapy, primary health care, physiotherapists 


\section{INTRODUCTION}

For patients with chronic low back pain (CLBP) various multidisciplinary biopsychosocial rehabilitation programmes have been developed showing moderate but promising effects. ${ }^{1}$ The general aim of such multidisciplinary biopsychosocial rehabilitation programme is to modify pain cognitions, to stimulate active coping behaviour, and to improve the level of functioning despite pain. Multidisciplinary biopsychosocial rehabilitation programmes are often protocolled and extensive. Patients with CLBP however differ in biopsychosocial profiles and it is therefore suggested that therapy (approach/content, intensity, setting) should be based on complexity of patient's complaints and patient's needs. ${ }^{2,3}$

In the Netherlands, the Task force Pain Rehabilitation of the Dutch Association for Rehabilitation Physicians (WPN-VRA) developed a classification system based on the complexity of the patient's psychosocial complaints. The classification is applied by physicians to refer a patient to the appropriate therapy setting. ${ }^{4}$ Mainly patients with moderate to high complex psychosocial complaints are referred to multidisciplinary biopsychosocial rehabilitation programmes in secondary or tertiary settings (i.e. hospitals, rehabilitation centres). The direct medical costs of these programmes are high, placing a significant burden on the healthcare system. ${ }^{5}$ In addition, the number of patients treated in secondary and tertiary settings has increased during the past years and it is expected that this number will increase even further. ${ }^{6-8}$

To control the increasing costs, substitution of biopsychosocial rehabilitation from a secondary to a primary care physiotherapy setting is a potential strategy. In the Netherlands, direct medical costs of primary care physiotherapy treatments are lower and waiting lists are shorter as compared to secondary care treatments. To what extent patients who usually receive treatments in a secondary care setting will benefit from a biopsychosocial intervention provided in a primary care setting, remains however to be investigated.

For this reason, a biopsychosocial primary care intervention ('Back on Track') has been developed in which therapy sessions are provided by a specifically trained physiotherapist. Patients are referred by a physician in rehabilitation medicine. This physician remains involved in the treatment and provides a final consultation at the end of the intervention. This results in an integrated care intervention for this subgroup of patients with moderate complex psychosocial complaints. Before evaluating the effectiveness of this newly developed intervention, it is important to investigate to what extent the intervention would be feasible in a primary care physiotherapy setting, e.g. in terms of expectancies, fidelity (quality), dose delivered (completeness), dose received (exposure), reach (participation rate), recruitment, and context (environmental factors). ${ }^{9}$ Having a clear overview of such process related factors will give insight to what extent the intervention is feasible to implement. It might, if desired or necessary, be useful to optimise the intervention for implementation, and will be useful for other researchers and clinicians who aim to develop, evaluate and/or implement a new biopsychosocial intervention.

The first aim of this study was to investigate to what extent the Back on Track intervention would be feasible in patients with CLBP who experience moderate complex biopsychosocial complaints. The second aim was to investigate to what extent these patients would benefit from 
the Back on Track intervention (i.e. reduce the level of functional disability). To combine these aims in one study, we initiated a process evaluation alongside an effect evaluation and formulated the following objectives:

1. To what extent attended physiotherapists the Back on Track training and were they satisfied with the training programme? (Reach \& dose received)

2. To what extent was the Back on Track intervention considered credible, and what were the expectancies of patients, physicians and physiotherapists prior to the start? (Credibility \& Expectancy)

3. To what extent attended patients the Back on Track intervention? (Reach)

4. To what extent was the Back on Track intervention delivered as planned? (Fidelity \& dose delivered)

5. To what extent were patients, physicians and physiotherapists satisfied with the Back on Track intervention? (Dose received)

6. To what extent had patient recruitment influenced the delivery of the Back on Track intervention? (Recruitment)

7. To what extent had barriers or facilitators experienced by physiotherapists and physicians influenced the delivery of the Back on Track intervention? (Context)

8. To what extent improved patients in functional disability after receiving the Back on Track intervention? (Effectiveness)

\section{MATERIALS AND METHODS}

This study included a mixed-methods design in which multiple qualitative and quantitative measurement instruments (e.g. questionnaires, focus groups, audio recordings), derived from different participants (patients, physicians and physiotherapists) were used to answer the research questions presented above. The Medical Ethics Committee of the University Hospital of Maastricht and Maastricht University, the Netherlands approved the study (METC143024), and the Clinical Trial Centre Maastricht (CTCM) monitored the study.

\section{PARTICIPANT \& SETTINGS}

Physicians in rehabilitation medicine working at Maastricht University Medical Centre (MUMC+, the Netherlands) referred patients with moderate complex biopsychosocial complaints to the Back on Track intervention (August 2014-May 2016). Patients were eligible if they fulfilled the following criteria; age between 18-65 years, LBP for $\geq 12$ weeks, sufficient knowledge of the Dutch language, no specific cause for CLBP, no suspicion of a (underlying) psychiatric disease, and no pregnancy. The complexity of the biopsychosocial complaints was determined by history taking and physical examination. If desirable, physicians used scores on multiple questionnaires although their own clinical opinion remained leading for classification. Range scores for questionnaires that could be used as guidance were determined based on clinical data (MUMC+) and existing datasets from previous conducted studies..$^{10,11}$ Range scores for a moderate complex bio- 
psychosocial profile included: Pain Catastrophizing Scale (PCS) between 21-23, Hospital Anxiety and Depression (HADS, each subscale) between 8-11, RAND-36 item Health Survey (RAND-36, physical functioning subscale) of > 35-42, and Pain Intensity Numeric Rating Scale (NRS, average pain last week) of $>4$. Eligible patients received pain education and were prepared for a biopsychosocial intervention by the referring physicians. Physicians furthermore informed (oral and written information) patients about study participation. If patients were willing to participate, patients gave written consent to be contacted by the research team.

The research team invited patients for an intake session at Maastricht University. After further information was provided about the study, patients gave written consent to participate in the study and completed baseline questionnaires. Patients additionally gave written consent to use the clinical questionnaires they completed previously to the first consultation with the physician. After the intake session, patients were referred to one of the participating physiotherapy practices.

In total four physiotherapy practices in the surroundings of Maastricht provided the Back on Track intervention. Practices were selected based on appropriate facilities (having a group therapy room), motivation and willingness to provide a biopsychosocial (group) therapy, as well as their willingness to participate in a scientific study and to receive a training programme. Each practice assigned two physiotherapists to deliver the Back on Track intervention. These physiotherapists were trained during 3 sessions ( 4 hours each) and received a treatment manual. Training sessions included basic knowledge on (chronic low back) pain, biopsychosocial factors, cognitive behavioural principles, and practical training regarding application of pain education and cognitive behavioural strategies in the physiotherapy setting. Physiotherapists received support during the study if needed and received two extra booster meetings (2 hours each) to discuss difficulties and to stimulate adherence during the study.

\section{THE BACK ON TRACK INTERVENTION}

The Back on Track intervention was developed by a team of physicians in rehabilitation medicine, physiotherapists, psychologists and patients. It was based on latest available evidence as well as on clinical experience from multidisciplinary pain rehabilitation teams at MUMC + and Adelante, Centre of Expertise in Rehabilitation and Audiology (Hoensbroek, The Netherlands). The Back on Track intervention aimed to improve the level of functional disability and to stimulate active coping behaviour. Reducing pain was not a direct goal. The intervention consisted of 12 sessions; 3 individual educative sessions (30 minutes each) at the start, 8 group sessions (60 minutes each) in between, and a final individual evaluation session (30 minutes). The role of the first individual sessions was to specify the individual pain problem (using the pain-consequence model) ${ }^{12}$, to educate about pain physiology and to define patient-specific goals. These individual sessions prepared patients for group sessions in which different cognitive-behavioural strategies were offered to stimulate active behaviour. Group sessions consisted of an educative part and a functional exercise part. Educative parts discussed dysfunctional beliefs, behaviour and strategies to improve the level of activities. In the functional exercise parts patients were stimulated to become 
physically active, to discuss patient's behaviour while being active, and to improve self-confidence. The group sessions were structured around four themes (pain \& physical activity, pain \& social network, pain \& cognitions, fact or myth?). A detailed description is provided elsewhere.12 Patients received a workbook including e.g. topics discussed during each session, assignments to reconsider own beliefs and behaviour, and self-management strategies to improve the level of activities at home. The Back on Track intervention ended with a final consultation of the referring physician. The physician evaluated the progress, reinforced behaviour change and provided information for long term adherence to active lifestyle.

\section{VARIABLES AND DATA COLLECTION}

Several measurement instruments, derived from different participants (patient, physicians and physiotherapists) were used to gather data and answer each research question (Table 1). This triangulation approach gives a comprehensive overview and detailed understanding of the feasibility of the Back on Track intervention.

\section{Attendance list Training programme (physiotherapists)}

Attendance lists were used to assess attendance of physiotherapists during the training programme. Sufficient compliance with the training programme was defined as having attended at least all three training sessions prior to delivery.

\section{Evaluation form training programme (physiotherapists)}

An evaluation form was used to investigate physiotherapists' experiences with the training programme. Physiotherapists completed the evaluation form directly after the third educational meeting. The evaluation form included questions about the level of the education/information, the competence of the educational team, and to what extent physiotherapists improved knowledge and skills. The evaluation form used a 6-point rating scale, ranging from 0 (very bad/too little) to 5 (very good/very much).

\section{Credibility and expectancy questionnaire}

An adapted version of the Dutch Credibility and Expectancy questionnaire (CEQ) was used to assess the credibility and expectancy of the Back on Track intervention. ${ }^{13}$ The questionnaire has good content validity, is easy to understand ${ }^{13}$, and scales have high internal consistency. ${ }^{14}$ Both patients and physiotherapists completed the CEQ directly after the first individual treatment session. The questionnaire included 5 questions for credibility and 6 for expectancies. Each item was answered with a 9-point rating scale ranging from 1 (not at all) to 9 (very much). Total score ranged from 5-45 for credibility and 6-54 for expectancy. A higher total score indicates a higher credibility or expectancy. 


\section{Attendance list Back on track intervention (patients)}

Attendance lists were used to assess attendance of patients during the Back on Track intervention and the final consultation with the physician. To our knowledge, no guidelines are available to define sufficient compliance for cognitive behavioural interventions. Therefore, we chose a cut off of having attended (at least) three individual sessions (session 1-3) and half of the group sessions $(n=4)$. We expected this number to be sufficient to understand and generalise learned cognitive behavioural principles.

\section{Audio recordings Back on track sessions}

Physiotherapists audio recorded Back on Track sessions to investigate to what extent physiotherapists delivered protocol elements as intended. A checklist based on the Method of Assessing Treatment Delivery (MATD) was used to assess treatment delivery..$^{15}$ The checklist included four types of elements; i.e. essential and unique (EU), essential but not unique (E), unique but not essential (U), and prohibited (P) (Table 4). Each element was rated as'satisfactorily achieved', 'partially achieved' or 'not achieved'. Sufficient treatment delivery was defined as having on average $\geq 70 \%$ of the EU and E elements satisfactorily achieved during therapy. Furthermore, less than 10\% of the $\mathrm{P}$ elements delivered during therapy was allowed. A trainee and physiotherapist in chronic pain (CK and JN) who were not involved in the study but experienced in applying cognitive-behavioural treatment principles independently rated the audio recordings. Raters were trained using audio recordings not selected for the final assessment. A total of 50 of the 127 available audio recordings (39\%) were used to assess treatment delivery. This number is sufficient to calculate interrater reliability as stated by COSMIN criteria. ${ }^{16}$ We choose to assess multiple sessions of an intervention due to the fact some physiotherapists did deliver elements not in the protocolled order. Seventy percent of the 50 audio recordings was used to assess complete treatment episodes (session 1-12) and 30\% to assess individual treatment episodes (session 1-3). This resulted in 3 entire treatment episodes assessed (35 of the 50 audio recordings), and 5 individual treatment episodes (15 of the 50 audio recordings), which were randomly chosen.

\section{Evaluation form back on track intervention}

A self-developed evaluation form was completed by patients directly after the last session with the physician. This evaluation form investigated patient's perceived knowledge, experiences and opinions about the intervention, the physiotherapist and the physician. The questionnaire included qualitative open-ended questions and quantitative rating scales.

\section{Focus groups}

Focus groups were organised at the end of the pilot study to gather the experiences of physicians and physiotherapists with the Back on Track intervention. One focus group was organised for participating physicians who referred patients to the pilot study ( 2 hours, MUMC,$+ n=4$ ). 
Another focus group was organised for participating physiotherapists who delivered the Back on Track intervention (2 hours, Maastricht University, $n=4$ ). Both focus groups were conducted by a senior researcher experienced in performing qualitative research (AK) and a student researcher. A semi-structured interview guide with open-ended questions regarding pre-specified themes (e.g. expectancy, credibility, dose delivered, dose received and practical issues) was used to investigate experiences during the Back on Track intervention. Participants were first asked to write down individually their experiences regarding pre-specified themes. Afterwards, individual notes were displayed, discussed in the group and clarified with in-depth questions. Both focus groups were audio recorded and subsequently transcribed by a student researcher and checked by a second researcher (RVE). The transcript was independently coded and thematised by both researchers, taking into account pre-defined process themes. Final codes and themes were discussed with a third researcher (AK) and eventually chosen consensus-based.

\section{Quebec Back Pain Disability Scale}

The Dutch version of the Quebec Back Pain Disability Scale (QBPDS) was used to investigate patient's improvement at functional disability level. ${ }^{17}$ Psychometric characteristics of the QBPDS are good. ${ }^{17-19}$ Patients completed the questionnaire at baseline, directly post-treatment and at 3 months follow-up (post-treatment). The QBPDS is a 20-itemed questionnaire with an answering scale from 0 (not difficult at all) to 5 (unable to perform). A total higher score indicates a higher level of functional disability.

\section{DATA ANALYSIS}

Normality of baseline data was assessed with the Shapiro-Wilk test. Means and standard deviations (SD) were calculated for normally distributed continuous baseline variables. Otherwise, medians and range scores were reported. Frequencies were calculated for nominal and ordinal baseline variables. Differences in baseline characteristics between patients who continued or discontinued the intervention were compared with a Chi-square test and Mann-Whitney test (non-parametric due to the small sample).

Additional analyses are described per research question. Research question 1: frequencies were calculated for physiotherapists' attendance, means and SD for ratings. Focus group data was analysed descriptively by thematic analysis (also applicable to research question 2, 4, 5, 6 and 7). Research question 2: credibility and expectancy of patients and physiotherapists were compared (per item and total score) with a Mann-Whitney $U$ test and reported as median, range. Total score credibility and expectancy of patients and therapists were separately added as covariate to the final multilevel model (described below) to determine the influential role on the QBPDS. Research question 3: frequencies were calculated for patients' attendance. Research question 4: ratings from both raters regarding the audio recordings of the Back on Track sessions were digitalised. Elements that could not be rated or were rated only once (such as sports activities), were deleted from the analysis. Inter-rater reliability of the raters was assessed using weighted Cohen's kappa to investigate the agreement. The average score of EU, E, $U$ and $P$ elements delivered by physio- 
therapists was calculated as the mean proportion achieved over all rated sessions. Frequencies were calculated for patients' ratings from the evaluation form. Research question 5, 6 and 7: Data concerned descriptive data, mean and SD for ratings. Research question 8: Multilevel analysis was used to investigate improvements in QBPDS over time (repeated measures, time as fixed factor and patient as random factor) using the identity covariance structure. Age, sex, duration of low back pain, and low back pain intensity at baseline were considered as covariates. Significance level was set at 0.05 .

\section{RESULTS}

\section{CHARACTERISTICS OF PARTICIPANTS}

Eighteen of the 23 eligible patients participated in the pilot study and received the Back on Track intervention (Figure 1). Participating patients were mainly women with a mean age of 45 years (range 18-59; Table 2). At group level, patients experienced moderate complex biopsychosocial complaints. At individual level, one patient was less complex than the predefined range off scores, while the others fulfilled at least 1 criterion (NRS cut off score $>4,94 \%$ ). Of these, 13 patients experienced one or more psychosocial complaints in addition (i.e. score within or above the predefined range scores). Eight patients discontinued the intervention. They had a significant lower median pain intensity at baseline of 6 (range 2-8) as compared to patient who continued the intervention (median 7, range 6-10). Two of the 18 patients were lost to follow-up.

Four physicians in rehabilitation medicine recruited patients for the pilot study. All physicians worked in chronic pain rehabilitation. The physicians' professional experience in chronic low back pain rehabilitation ranged from 5-19 years. At the end of the study, all four physicians attended the focus group.

In total twelve physiotherapists were educated to deliver the Back on Track intervention. Although education started with two physiotherapists per practice $(n=8)$, two physiotherapists quitted their job, one went on maternity leave and one seemed less available to deliver treatment. Therefore, four extra physiotherapists needed to be educated during the trial. Of the total educated physiotherapists, six were male, six female. The professional experience ranged from recently being graduated to 31 years. Finally, four physiotherapists delivered the Back on Track intervention (three males, one female; graduated recently $(n=1)$, 4 years of experience $(n=2)$, and 31 years of experience $(n=1)$. Three of the four physiotherapists delivering the intervention attended the focus group. 


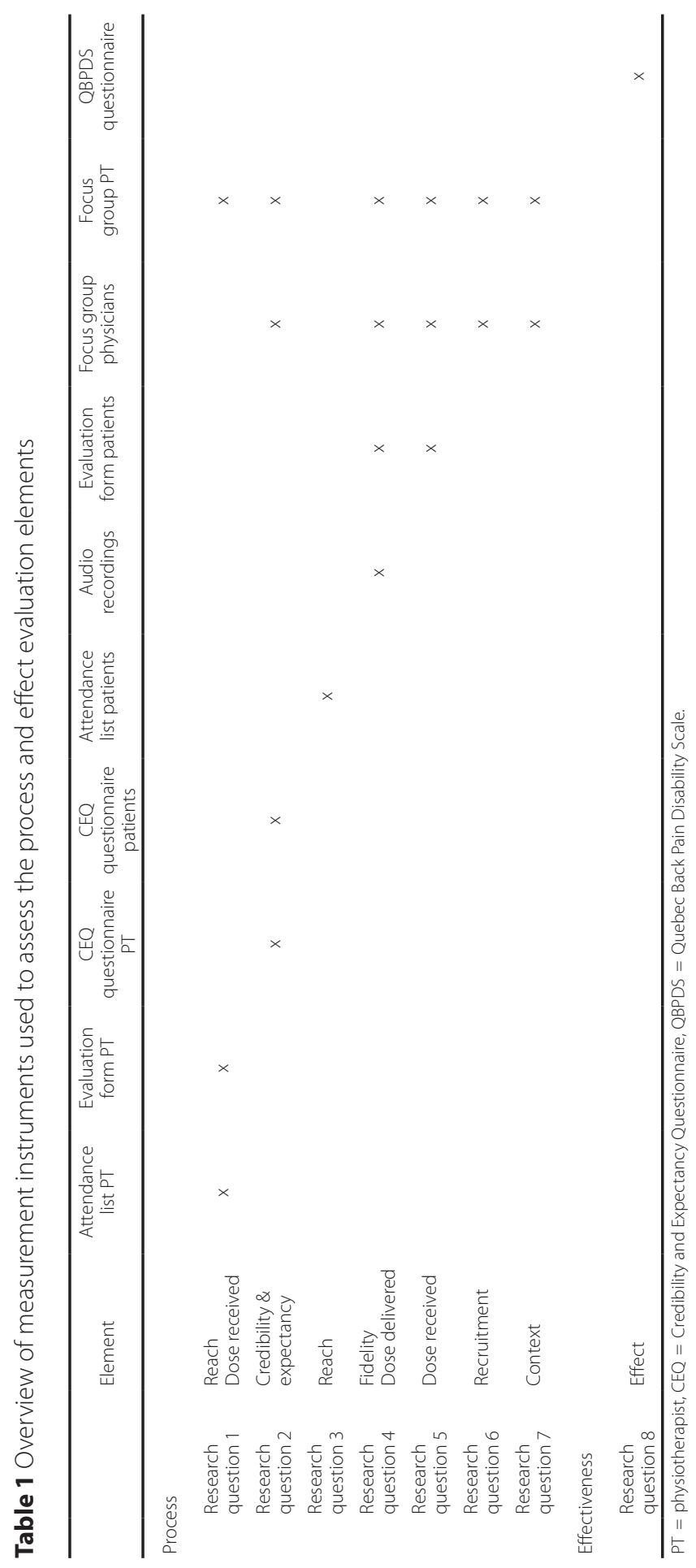


Figure 1 Flow chart of the study participants

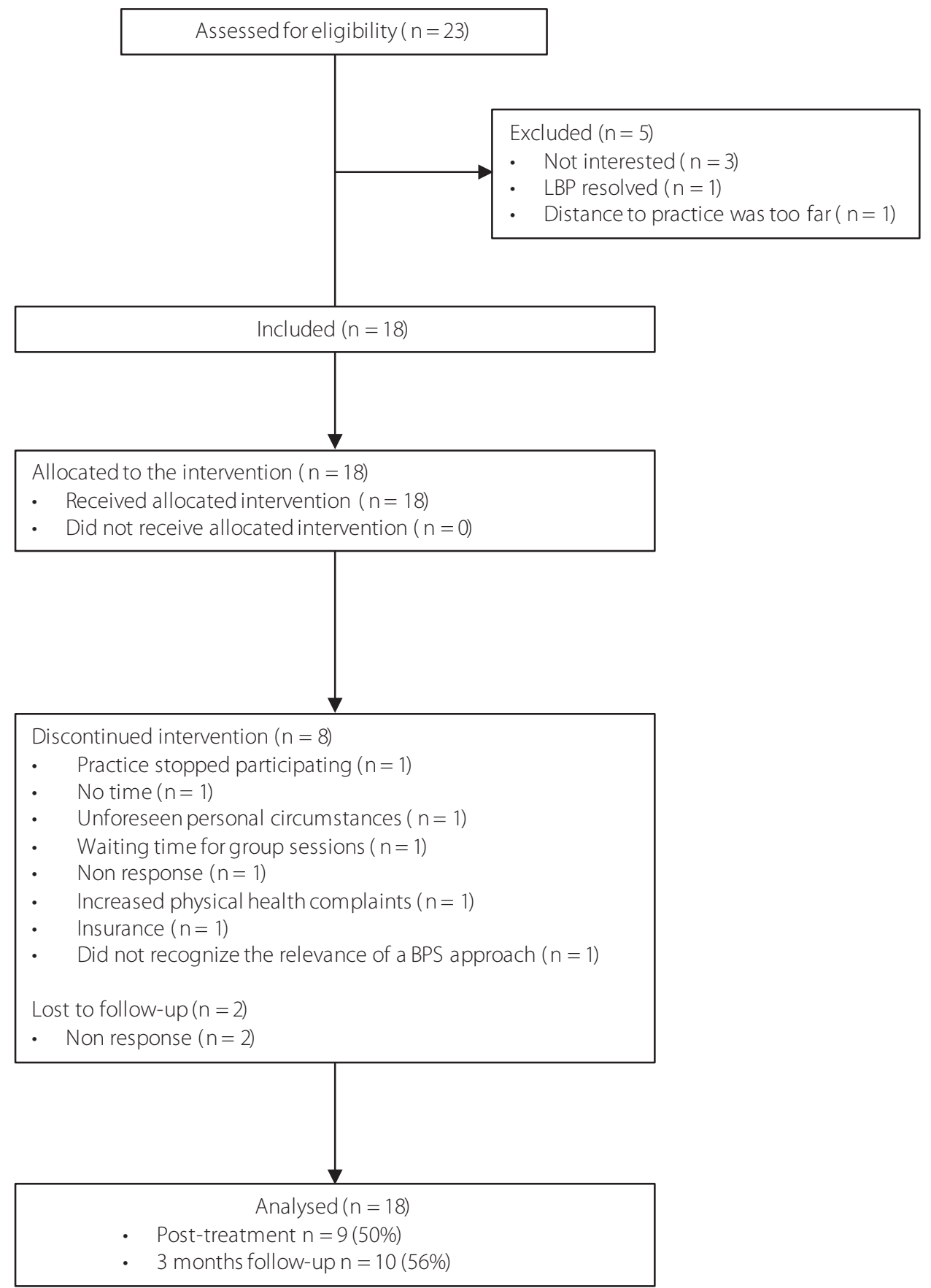


Table 2 Baseline characteristics of study participants

\begin{tabular}{|c|c|}
\hline & $\begin{array}{l}\text { Back on Track intervention }(n=18) \\
\text { Mean/frequency }(S D / \%)\end{array}$ \\
\hline Age (years)* & $49(18-59)$ \\
\hline Sex (female) & $13(72 \%)$ \\
\hline \multicolumn{2}{|l|}{ Nationality } \\
\hline Dutch & $16(89 \%)$ \\
\hline Other & $2(11 \%)$ \\
\hline \multicolumn{2}{|l|}{ Education level } \\
\hline Low & $9(50 \%)$ \\
\hline Middle & $7(39 \%)$ \\
\hline High & $2(11 \%)$ \\
\hline \multicolumn{2}{|l|}{ Employment } \\
\hline Full-time & $2(11 \%)$ \\
\hline Part-time & $1(6 \%)$ \\
\hline Full-time education & $2(11 \%)$ \\
\hline Voluntary work & $8(44 \%)$ \\
\hline Unemployed & $5(28 \%)$ \\
\hline \multicolumn{2}{|l|}{ Duration of low back pain (since first onset) } \\
\hline $3-6$ months & $1(6 \%)$ \\
\hline $6-12$ months & $3(17 \%)$ \\
\hline $1-2$ years & $2(11 \%)$ \\
\hline $2-5$ years & $7(39 \%)$ \\
\hline$>5$ years & $5(28 \%)$ \\
\hline Functional disability (QBPDS) & $42(14.0)$ \\
\hline Functional disability (PDI) & $33(10.6)$ \\
\hline Pain intensity (NRS)* & $6(2-10)$ \\
\hline Pain catastrophizing (PCS) & $21(8.3)$ \\
\hline \multicolumn{2}{|l|}{ Anxiety and Depression (HADS) } \\
\hline Anxiety* & $6(1-20)$ \\
\hline Depression* & $5(1-14)$ \\
\hline Physical functioning (RAND-36, subscale) & $50(15.3)$ \\
\hline Mental health (RAND-36, subscale) & $66(18.1)$ \\
\hline Pain self-efficacy (PSEQ) & $37(9.9)$ \\
\hline Kinesiophobia (TSK) & $37(5.9)$ \\
\hline \multicolumn{2}{|c|}{$\begin{array}{l}\text { QBPDS = Quebec Back Pain Disability Scale }(0-100) ; \text { PDI = Pain Disability Index (0-70); NRS = Numeric Rating Scale (0-10), } \\
\text { average pain last week; PCS = Pain Catastrophizing Scale (0-52); HADS = Hospital Anxiety and Depression Scale (0-21 per } \\
\text { subscale); RAND-36 = RAND-36 item Health Survey (0-100 per subscale); PSEQ = Pain Self Efficacy Questionnaire (0-60);TSK = } \\
\text { Tampa Scale for Kinesiophobia (17-68) } \\
\text { *Data is presented as median (range) due to the asymmetric distribution. }\end{array}$} \\
\hline
\end{tabular}


1. To what extent attended physiotherapists the Back on Track training and were they satisfied with the training programme? (Reach and dose delivered)

All physiotherapists attended all three Back on Track training sessions $(n=12)$. According to the evaluation form, therapists who delivered the intervention eventually ( $n=4)$ as well as those who did not $(n=8)$ evaluated the level of education as good (respectively $4.0 \pm 0.0$ and $4.4 \pm$ 0.5 ) and the competence of the education team near very good (respectively $4.8 \pm 0.5$ and 4.6 \pm 0.5 ). Physiotherapists gained new knowledge (respectively $3.8 \pm 0.5$ and $3.9 \pm 0.7$ ), new skills (respectively $3.5 \pm 0.6$ and $3.3 \pm 0.5$ ), and considered the training relevant for practice (respectively $3.8 \pm 1.3$ and $4.4 \pm 0.5$ ). Physiotherapists who delivered the intervention confirmed during the focus group that they were satisfied with the training programme.

2. To what extent was the back on track intervention considered as credible, and what were the expectancies of patients, physicians and physiotherapists prior to the start?

During the focus group, physiotherapists as well as physicians reported that they considered the Back on Track intervention, prior to the start, a credible and all-embracing intervention as it included multiple logical themes, psychosocial elements and group sessions. One physiotherapist expressed concerns regarding the duration of the second individual session (pain-education); whether there would be sufficient time to anticipate on the individual's situation and questions. Furthermore, another physiotherapist questioned whether it would be feasible to provide exposure in vivo elements and thought it would better fit psychologists.

Regarding the CEQ questionnaire, patients and physiotherapists did not differ in credibility and expectancy items ( $p>0.05$ ), except for one aspect; patients had significantly greater expectations that the Back on Track would reduce the level of pain than physiotherapists (median, range score of 5.0, $1-8$ versus $2.5,2-7$, respectively). Finally, patients' expectancy and credibility scores did not significantly influence the improvements in functional disability.

In the focus groups, physiotherapists and physicians both noticed that some patients did not know what to expect or still had different expectations of the therapy (e.g. more exercise therapy, less talking).

\section{To what extent attended patients the Back on Track intervention? (Reach)}

The mean number of sessions the patients attended during the Back on Track intervention was 8.3 \pm 4.1 (range 2-12). In total, $67 \%$ of the patients met the compliance threshold. Attendance rates per session show high attendance during individual sessions (session 1-3) and lower attendance during group sessions (Table 3). Lower attendance was due to discontinuance of the intervention $(n=6)$, holiday $(n=1)$, or unknown reasons $(n=4)$. Reasons for discontinuing the intervention are listed in Figure 1. After the intervention, 10 out of 18 attended the final consultation with the physician. No session was planned for two patients (unknown reason), four did not show up (unknown reason), and one cancelled (patient reported that he did not need it). 
Table 3 Attendance rates and mean satisfaction by patients per session of the Back on Track intervention

\begin{tabular}{lcc}
\hline Session & $\begin{array}{c}\text { Attendance rate } \\
\mathrm{n}(\%)\end{array}$ & $\begin{array}{c}\text { Satisfaction rating } \\
\text { Mean } \pm \text { SD }(\mathrm{n})\end{array}$ \\
\hline Individual session 1 & $18(100 \%)$ & $7.6 \pm 1.0(\mathrm{n}=11)$ \\
Individual session 2 & $18(100 \%)$ & $7.2 \pm 0.9(\mathrm{n}=11)$ \\
Individual session 3 & $16(89 \%)$ & $7.6 \pm 1.2(\mathrm{n}=10)$ \\
& & \\
Theme 1, session 1 & $13(72 \%)$ & $7.3 \pm 1.5(\mathrm{n}=7)$ \\
Theme 1, session 2 & $12(67 \%)$ & $6.3 \pm 2.9(\mathrm{n}=7)$ \\
Theme 2, session 1 & $11(61 \%)$ & $7.5 \pm 1.5(\mathrm{n}=6)$ \\
Theme 2, session 2 & $12(67 \%)$ & $6.3 \pm 2.9(\mathrm{n}=7)$ \\
Theme 3, session 1 & $12(67 \%)$ & $6.6 \pm 2.0(\mathrm{n}=8)$ \\
Theme 3, session 2 & $11(61 \%)$ & $7.4 \pm 1.3(\mathrm{n}=7)$ \\
Theme 4, session 1 & $10(56 \%)$ & $7.2 \pm 1.0(\mathrm{n}=6)$ \\
Theme 4, session 2 & $6(33 \%)$ & $5.8 \pm 3.9(\mathrm{n}=4)$ \\
& & \\
Individual session 4 & $10(56 \%)$ & $6.5 \pm 3.4(\mathrm{n}=6)$ \\
& & \\
Individual consultation physician & $11(61 \%)$ & $5.4 \pm 4.0(\mathrm{n}=7)$
\end{tabular}

4. To what extent was the Back on Track intervention delivered as planned? (Fidelity \& dose delivered)

Physicians mentioned during the focus group that they did not have insight into the extent physiotherapists adhered to the protocol and/or sufficiently provided cognitive-behavioural elements. Physiotherapists themselves reported that deviations from the protocol only occurred occasionally in case e.g. groups could not be formed. Two physiotherapists admitted they had once merged two sessions into one because the group was small.

According to the audio recordings, EU and E elements were on average for satisfactorily achieved in $80 \%$ ( \pm 16.7), indicating sufficient protocol adherence (Table 4). Interrater reliability between raters was fair (weighted Cohen's kappa $=0.489$ ). Those EU elements that were $<70 \%$ satisfactorily achieved were mainly group session elements. One prohibited element occurred in 15\% of the rated sessions ('the use of medical diagnosis to explain or justify the decreased level of daily life functioning'). This mostly occurred during theme 4 in which facts and myths about low back pain were discussed.

Considering specific protocol elements, physiotherapists seemed to be able to optimally discuss a patient's LBP history, to define important functional activities and set a specific plan to enhance daily activity levels (Graded activity; mean 100\%, Table 4). During the focus group, physiotherapists reported that especially Graded Activity elements had a clear structure and therefore went well. They mentioned that as soon as the topic became more personal and patients needed to speak about unpleasant/negative situations, emotions or cognitions, patients waited more for other group members to respond. According to the audio recordings, discussions about the 
vicious circle of negative thoughts and the short and long term consequences of a patient's pain-behaviour were some elements that were occasionally not addressed. Most patients however still reported that they learned about how thoughts and emotions can influence coping behaviour (8/10), and that physiotherapists gave the opportunity to discuss their cognitions and emotions (9/11).

Defining the level of perceived threat value of daily activities was one element occasionally not addressed during therapy. One physiotherapist addressed however some patients did not recognise themselves in having these fearful thoughts and avoidance behaviour. It became clear from the patient evaluation forms that only half of the patients would avoid or compensate fewer activities in future (4/10). Five patients responded neutral, and one slightly disagreed (i.e. would not avoid/compensate less in future).

Physical active sessions were largely not recorded and could therefore not be assessed by raters. According to physiotherapists' own experiences, physical active sessions focused on activities to perform or improve patient-specific goals what was in line with the protocol. Physiotherapists however noticed it was not always possible to actually improve the level of the activity during the session due to the limited time available. One physiotherapist explained to his patients that physical active sessions were meant to improve the quality of activities and to reinforce behaviour, and that patients needed to improve the level of activity (intensity/duration) themselves in their home situations. This was in line with the protocol.

Physiotherapists reported that few patients (slightly) deviated from the protocol as they did not always perform homework assignments addressed in the workbook. Two of the 10 patients reported themselves to have read less than half of the workbook. Eight patients had read at least half of the workbook of which four had read it entirely. Physiotherapist considered it however more important that patients would understand the information of the workbook, and could explain it themselves (realising what they were doing), rather than just completing assignments. Over half of the patients (6/11) agreed at the end of the intervention that they would be able to improve the level of activities themselves. Four patients responded neutral, and one patient disagreed. 
Table 4 Percentage of elements achieved during therapy sessions according to the ratings of audio recordings

\begin{tabular}{|c|c|c|c|c|c|}
\hline Session & Item & Element & $\begin{array}{l}\text { Not } \\
\text { achieved } \\
(\%)\end{array}$ & $\begin{array}{l}\text { Partially } \\
\text { achieved } \\
(\%)\end{array}$ & $\begin{array}{l}\text { Satisfactorily } \\
\text { achieved (\%) }\end{array}$ \\
\hline Individual 1-3 & $\begin{array}{l}\text { Aim of intervention is explained (improving } \\
\text { functional disability level + coping style) }\end{array}$ & EU & 6 & 6 & 88 \\
\hline Individual 1-3 & Pain education is provided & EU & 0 & 6 & 94 \\
\hline Individual 1-3 & Pain-consequence model is discussed & EU & 0 & 13 & 88 \\
\hline Individual 1-3 & Patient specific functional activities are defined & EU & 0 & 0 & 100 \\
\hline Individual 1-3 & SMART goals are set & EU & 0 & 14 & 86 \\
\hline Theme 1 & $\begin{array}{l}\text { Short and long term consequences of } \\
\text { pain behaviour are discussed }\end{array}$ & EU & 17 & 33 & 50 \\
\hline Theme 1 & Graded Activity principles are discussed & EU & 0 & 33 & 67 \\
\hline Theme 1 & Disadvantages of pain behaviour are discussed & EU & 0 & 17 & 83 \\
\hline Theme 1 & The aim of Graded Activity is discussed & EU & 0 & 17 & 83 \\
\hline Theme 1 & The baseline activity level of the patient is discussed & EU & 0 & 0 & 100 \\
\hline Theme 1 & A Graded Activity plan is defined & EU & 0 & 0 & 100 \\
\hline Theme 1, 2, 4 & Vicious circle is discussed (influence of negative thoughts) & EU & 11 & 33 & 56 \\
\hline Theme 2 & The coping style of patient is discussed & EU & 0 & 17 & 83 \\
\hline Theme 2 & $\begin{array}{l}\text { The coping style of environment } \\
\text { (family/work) is discussed }\end{array}$ & EU & 0 & 0 & 100 \\
\hline Theme 2 & Factors that influence behaviour change are discussed & EU & 0 & 33 & 67 \\
\hline Theme 3 & $\begin{array}{l}\text { Concerns/fear regarding activities is } \\
\text { discussed (e.g. using the PHODA) }\end{array}$ & EU & 0 & 33 & 67 \\
\hline Theme 3 & $\begin{array}{l}\text { The level of perceived threat value } \\
\text { of daily activities is defined }\end{array}$ & EU & 17 & 33 & 50 \\
\hline Theme 4 & $\begin{array}{l}\text { Facts and myths about chronic (low) } \\
\text { back pain are discussed }\end{array}$ & EU & 0 & 17 & 83 \\
\hline Theme 4 & $\begin{array}{l}\text { The influence of positive and negative } \\
\text { thoughts and emotions are discussed }\end{array}$ & EU & 0 & 0 & 100 \\
\hline Individual 4 & $\begin{array}{l}\text { To what extent the patient has insight into the } \\
\text { influencing psychosocial factors is discussed }\end{array}$ & EU & 17 & 33 & 50 \\
\hline Individual 4 & $\begin{array}{l}\text { To what extent the patient has insight into } \\
\text { self-management strategies to improve } \\
\text { the level of activities is discussed }\end{array}$ & EU & 0 & 33 & 67 \\
\hline Individual 4 & Future policy is discussed/defined & EU & 0 & 17 & 83 \\
\hline Session & Item & Element & $\begin{array}{c}\text { Not } \\
\text { achieved } \\
(\%)\end{array}$ & $\begin{array}{l}\text { Partially } \\
\text { achieved } \\
\text { (\%) }\end{array}$ & $\begin{array}{l}\text { Satisfactorily } \\
\text { achieved (\%) }\end{array}$ \\
\hline Individual 1-3 & $\begin{array}{l}\text { The therapist goes into the chronic pain symptoms } \\
\text { (existence, course, status praesens), level of activity and } \\
\text { participation, previous treatments and/or diagnosis }\end{array}$ & $E$ & 0 & 0 & 100 \\
\hline All & The patient is actively involved during the therapy & $E$ & 0 & 15 & 85 \\
\hline Individual 4 & Patient's experiences with the intervention are discussed & $E$ & 0 & 17 & 83 \\
\hline Individual 4 & $\begin{array}{l}\text { Patient's status and improvement during } \\
\text { the intervention is discussed }\end{array}$ & $\mathrm{E}$ & 0 & 33 & 67 \\
\hline
\end{tabular}




\begin{tabular}{|c|c|c|c|c|c|}
\hline Session & Item & Element & $\begin{array}{l}\text { Not } \\
\text { achieved } \\
(\%)\end{array}$ & $\begin{array}{l}\text { Partially } \\
\text { achieved } \\
(\%)\end{array}$ & $\begin{array}{l}\text { Satisfactorily } \\
\text { achieved (\%) }\end{array}$ \\
\hline All & $\begin{array}{l}\text { It is discussed that decreasing pain is } \\
\text { not a goal of the intervention }\end{array}$ & U & 28 & 15 & 57 \\
\hline All & $\begin{array}{l}\text { The therapist elicits influencing cognitions, } \\
\text { feelings, emotions regarding pain or activities }\end{array}$ & U & 4 & 20 & 76 \\
\hline Theme 3 & $\begin{array}{l}\text { Important factors are discussed that } \\
\text { influenced exposure to fearful activities (e.g. } \\
\text { safety behaviour, verbal expression) }\end{array}$ & U & 33 & 33 & 33 \\
\hline Session & Item* & Element & $\begin{array}{l}\text { Not } \\
\text { achieved } \\
(\%)\end{array}$ & $\begin{array}{l}\text { Partially } \\
\text { achieved } \\
(\%)\end{array}$ & $\begin{array}{l}\text { Satisfactorily } \\
\text { achieved (\%) }\end{array}$ \\
\hline $\begin{array}{l}\text { Individual 1-3 } \\
\text { Theme 1-4 }\end{array}$ & $\begin{array}{l}\text { Fear/concern with regard to pain or } \\
\text { activities is expressed by the therapist }\end{array}$ & $P$ & 91 & 7 & 2 \\
\hline All & $\begin{array}{l}\text { Medical diagnoses are used to explain } \\
\text { or justify the decreased level of daily life } \\
\text { functioning (except for pain physiology) }\end{array}$ & $P$ & 85 & 13 & 2 \\
\hline
\end{tabular}

$\overline{E U}=$ essential and unique; $\mathrm{E}=$ essential but not unique; $\mathrm{U}=$ unique but not essential; $\mathrm{P}=$ prohibited; $\mathrm{PHODA}=$ Photograph Series of Daily Activities, measurement instrument to identify the perceived harmfulness of daily activities by patients. ${ }^{20}$

* For these items, it is desirable to have high percentages 'not achieved' (i.e. did not occur).

5. To what extent were patients, physicians and physiotherapists satisfied with the Back on Track intervention? (Dose received)

According to the evaluation form, patients reported general positive experiences with the Back on Track intervention such as the good conversations with a laugh and a tear, the understanding and humanity, the focus on both physical and mental factors and thereby learning a lot about pain and also themselves. During the focus groups, physiotherapists were also generally positive about the Back on Track intervention. Patients' negative experiences were that the time for talking was too long and for treatment too short, groups were too small and the time between the sessions was too long. Both physiotherapists and physicians agreed that the waiting time between individual and group sessions was long.

At the evaluation form, patients valued the individual sessions (session 1-3) on average higher than group sessions (respectively $7.5 \pm 1.0$ and $6.8 \pm 2.1$ ). Physiotherapists specifically addressed that pain education provided in individual sessions was most valuable of the Back on Track intervention and acted as the foundation for the rest of the intervention. Physiotherapists mentioned that the pain-consequence model used within the (educative) individual sessions was a valuable tool to define and understand a patient's situation. Physiotherapists stressed however that sufficient understanding by the patient was required and this was a prerequisite for improvements during therapy. Physiotherapists reported that they noticed afterwards who would respond best to the intervention; i.e. those patients who were aware of the situation and were willing to change. Two physiotherapists drew explicit attention to the fact they considered educative discussions within group sessions also more important than performing physical activities for which often less time was available. Patients however expected more physical activities and confirmed that physical activities were not always performed during physical active sessions. This might have caused patients to value the physical active group session slightly lower than the 
educative group sessions (respectively $6.5 \pm 2.6$ and $7.1 \pm 1.5$. Physicians furthermore noticed that patients overall experienced group sessions differently as some did not like group sessions at all, while others were very positive and ventilated to have learnt from group members.

The final consultation with the physician was scored unsatisfactory (Table 3). On the other hand, patients rated the physician in person on average a $7.9 \pm 2.2(n=10)$.

\section{To what extent had patient recruitment influenced the delivery of the Back on Track intervention? (Recruitment)}

Physicians and physiotherapists both agreed during the focus groups that the recruitment rate was disappointing. Patients were often older than inclusion criterion, did not live in the surrounding of the four physiotherapy practices or experienced psychosocial factors that were too complex. As a consequence, one physiotherapy practice decided to stop at the beginning of the study, leaving three participating physiotherapy practices.

Due to the low recruitment rate, patients reported that they needed to wait before groups could be formed and group therapy could start. They reported that this negatively influenced their motivation. Physiotherapists agreed on this and reported that if the waiting time took too long, groups were started with two patients only (with approval from the study team). Physiotherapists mentioned it was a challenge with small groups to stimulate interactive group discussions. One physiotherapist mentioned that group sessions turned into lectures sometimes. One physician stressed his concerns and questioned whether group sessions actually had achieved their maximal quality.

7. To what extent had barriers or facilitators experienced by physiotherapists and physicians influenced the delivery of the Back on Track intervention? (Context)

Approach of physicians at the first consultation

During the focus group with physicians it became evident that the complexity of the patient's biopsychosocial profile was largely based on history taking and less on questionnaire scores for which predefined range scores were available. Physicians drew attention to the fact questionnaire scores did not always reflect the actual status of the patient. Factors that were, in addition to psychosocial factors, deemed important for diagnoses were motivation, personal goals, whether the patient already received cognitive behavioural treatments, medication use, psychiatric problems, and patient's behaviour throughout the consultation and physical examination (e.g. moan and groan).

During the focus group, physicians also noticed that they managed the first consultation differently. Although physicians used similar pain-education elements (e.g. explanations about acute and chronic pain, central sensitization and the message that 'hurt does not mean harm'), one physician provided pain-education as one package after history taking and physical examination, while others mixed these elements and started pain-education directly, based on what the patient reported. 
Competence of physiotherapists

Physiotherapists reported that the theory during the education programme was not difficult, but implementation was more challenging. Although physiotherapists considered the training appropriate and the treatment manual as additional support very useful, they agreed that they got confronted with new complex situations. This was mainly the case when patients asked critical questions beyond the scope of the intervention. One physiotherapist noticed that therapists in general might need some experience to manage complex situations (and interactions) more easily.

\section{Communication}

Physicians and physiotherapists mentioned that communication between them occurred few times by email and never by phone. It often included logistic questions about how to deal with the waiting time for groups. Physicians would have appreciated more contacts as well as more in-depth reports from physiotherapists as this would give insight in the type of therapy patients received. Physiotherapists considered the reports from physicians extensive and good, but mentioned that it was unclear what own reports should include as this was not protocolled. Physiotherapists would appreciate guidelines for reporting in future (i.e. regarding content). Physiotherapists furthermore reported that interim contacts with the physician might be ideal in future although they questioned whether this would be feasible.

\section{Protocol in Primary care}

Physiotherapist mentioned that a protocolled intervention requires certain planning. Physiotherapists sometimes felt pressure to discuss all relevant topics in the specified timeframe. This was mainly applicable to individual session 2 in which pain education had to be delivered within 30 minutes. Physiotherapists agreed that this was sometimes too limited. Physiotherapist furthermore mentioned that the planning of group sessions, in contrast to individual sessions, was most challenging. Physiotherapists had to deal with the low recruitment/referral rate, working patients, and the (in-)flexibility of the practice.

8. To what extent improved patients in functional disability after receiving the Back on Track intervention?

Of the considered covariates, only low back pain intensity was of significant influence and therefore included in the final model. Mixed model analyses showed that patients statically significantly decreased the level of functional disability with on average 8.3 points $(95 \% \mathrm{Cl}-13.9$ to -2.7 ) (Table 5). At 3 months follow-up, the reduction in functional disability remained constant and therefore statistically significant as compared to baseline. 
Table 5 Estimates of improvements in functional disability at post-treatment and 3 months post-treatment

\begin{tabular}{ccc}
\hline & $\begin{array}{c}\text { Mean change score from } \\
\text { baseline* }(95 \% \mathrm{Cl}) \\
\mathrm{n}=18\end{array}$ & p-value \\
\hline QBPDS (points) & & .006 \\
Post-treatment & $-8.3(-13.9$ to -2.7$)$ & .009 \\
3 months & $-7.6(-12.9$ to -2.2$)$ & $.2)$ \\
\hline
\end{tabular}

* Baseline QBPDS was $42 \pm 14.0$.

\section{DISCUSSION}

\section{SUMMARY OF MAIN OUTCOMES}

In this pilot study we evaluated a newly developed integrated care intervention with a biopsychosocial approach (the Back on Track intervention) in primary care. The intervention was applied to a group of patients with moderate complex CLBP who were usually treated in a secondary care setting. This study showed that the Back on Track intervention was feasible in primary care physiotherapy practices and showed statistically significant improvements in the level of functional disability at short and medium term.

This process evaluation showed that the training programme for primary care physiotherapists with different levels of professional experience was feasible and resulted in sufficient delivery of essential protocol elements during therapy. Trained physiotherapists seemed to use medical diagnosis to explain or justify the decreased level of daily life functioning as well, which could indicate contamination with a biomedical approach. However, the use of this element mostly occurred during theme 4 in which biomedical facts and myths about low back pain was discussed. It remains unclear how the prohibited element was used; i.e. with a biomedical view/ approach (indicating contamination), or to confirm the need for a biopsychosocial approach (indicating no contamination). When deleting theme 4 from the analysis, the contamination with a biomedical approach was no longer present. For this reason, we assume that the primary care physiotherapists were able to sufficiently deliver the biopsychosocial intervention.

Of all sessions, the individual sessions were appreciated most by patients and physiotherapists, and especially session 2 (by physiotherapists) in which pain education was provided. The pain-consequence model was furthermore considered a powerful tool to tackle biomedical beliefs and to direct into a biopsychosocial orientation. Changing the function of beliefs by challenging them (i.e. exposure in vivo elements), on the other hand, was sometimes difficult and/ or less applicable. Exposure in vivo focuses on catastrophizing thoughts, pain-related fear and avoidance behaviour. ${ }^{21}$ Some patients however reported low levels of catastrophizing thoughts at baseline (PCS range score was 6-37) and furthermore did not recognise themselves in having pain-related fear or avoidance behaviour. 
Overall, patients attended the first individual sessions of the Back on Track intervention sufficiently, although their attendance dropped as soon as the group sessions started. The lower attendance was likely a consequence of the low recruitment rate causing an impaired group formation and increased waiting time to continue the programme. The waiting time decreased motivation and eventually the attendance rate during groups. In addition, patients expected more physical activities during group sessions. Physiotherapists found it more important to pay attention to the educational discussions and considered the time usually too limited to actually improve the level of activities. Performing no or less physical activities during therapy was not in line with patients' expectations of the primary care intervention. This might have decreased patients' motivation to some extent, and may have influenced attendance rates in turn.

Patients showed statistically significant improvements the level of disability after the intervention and at three months follow up. These results are in line with results from the Back Skills Training Trial in which patients with comparable biopsychosocial profiles significantly reduced the level of disability after a biopsychosocial primary care intervention at post treatment and follow-up.22 No causality could however be established with our used pre-post-test design without a control group. Nevertheless, it was a first study to identify the potency of a new approach that eventually showed positive effects.

\section{IMPLICATIONS FOR FUTURE}

Our findings are in line with a recently performed systematic review which revealed that physiotherapists can effectively deliver a biopsychosocial intervention if they are additionally trained and have resources available such as a treatment manual. ${ }^{23}$ Although no guidelines are available for optimal training of therapists, it would be recommended discussing multiple cases and the use of role playing during the training programme (as we did). Furthermore, booster sessions after training may be useful as well as providing feedback during delivery of the intervention (coaching on the job). ${ }^{24}$ This might further improve the cognitive-behavioural skills and confidence of physiotherapists and therefore optimise delivery.

Previous studies have shown that group therapy favours individual therapy as it can be cost-effective ${ }^{25}$ and stimulates patient interaction and societal integration. ${ }^{26}$ Although we recognise the potential advantages, we encountered practical difficulties with generating groups. Consequently, this resulted in lower satisfaction in patients. It should be stressed that, although group therapy might be preferred, it is recommended to provide group therapy only if recruitment is appropriate and groups can be generated in time.

The Back on Track intervention may furthermore use some slight adaptations in future. First, attention should be paid to the (rather subjective) approach of physicians to classify patients on the influencing role of psychosocial complaints. Clinical tools for secondary care are not yet available but may be useful in future. Second, more time should be incorporated for pain education as 30 minutes was considered too limited. Devoting two individual sessions to pain education would be in line with a scientifically published practice guideline for pain (physiology) education. ${ }^{27}$ Third, whether an exposure session (theme 3, session 2 ) needs to be provided in the 
protocolled format, should be dependent on the patient's psychosocial complaints. If a patient has less or no dysfunctional beliefs about pain and daily activities, a regular Back on Track physical active session may suffice. However, as during all sessions, physiotherapists have to observe the patient's behaviour and potential adaptations in this behaviour because of pain should be discussed. Fourth, physiotherapists should be encouraged to stick to the prespecified time to perform physical activities. Performing physical activities add significant benefits when added to education alone (i.e. significant reductions in pain). ${ }^{28}$ Furthermore, performing physical activities is in line with patients' expectations and probably will increase therapy satisfaction. Fifth, communication and collaboration between physiotherapists and physicians in rehabilitation medicine need to be improved to ensure an integrated care approach. A contact moment could be added halfway the intervention for physiotherapists and/or patients with physicians; e.g. to discuss the applied approach, to define future approaches and to integrate specialist's knowledge into primary practice. A format could furthermore be developed for physiotherapists with topics essential to report, based on the expert opinion of physiotherapists and physicians. Finally, a long term follow-up session might be beneficial to maintain or improve activity levels of patients, especially for those patients who are not entirely sure about their ability to do this individually at home (such as few patients reported in the study). A booster session could be used to define the level of improvement/deterioration, to rehearse approaches learnt, and if necessary, to refer to a secondary care intervention. It should be noted that almost half of our patients did not attend the final consultation with the physician. Therefore, it should be determined to what extent patients consider a follow-up session valuable, whether they will attend such session and which health care professional should provide this session.

The promising effects of the Back on Track intervention on improving the level of functional disability indicate the need for a future study to evaluate the differences in cost-effectiveness between a biopsychosocial primary care intervention and multidisciplinary (secondary) care as usual. Therapy sessions of a primary care physiotherapy intervention are generally less expensive than multidisciplinary (secondary) therapy sessions. A cost-effectiveness study is therefore expected to give direction to whether care for this subgroup of patients should be substituted to primary care settings.

\section{STRENGTHS \& LIMITATIONS}

A major strength of this study was the use of multiple quantitative and qualitative measurement methods as well as multiple sources (physicians, physiotherapists and patients). We believe that this led to detailed insight in multiple factors that influenced the implementation of an integrated care programme into daily practice. Researchers and health care providers could benefit from such process related information as it can be useful for replication, improving, and implementing a biopsychosocial intervention into routine clinical practice.

One limitation of the study could be the selection of physiotherapists which was not based on the biopsychosocial orientation. Literature shows that therapist's orientation (biomedical versus biopsychosocial) can influence therapy delivery and the advice they provide. 29,30 Our physio- 
therapists were however motivated to provide a biopsychosocial intervention and were offered a training programme to guide into a more biopsychosocial orientation. Although not evaluated afterwards, physiotherapists' orientation was considered sufficient as they delivered biopsychosocial protocol elements sufficiently.

Another limitation was the rather low interrater reliability between the two raters of the audio recordings. This was likely a consequence of the rating procedure, i.e. using three options instead of two. A three-point rating scale was considered more valuable as this would provide insight whether elements were provided and to what extent. Such information would be useful to optimise the intervention and the training programme for physiotherapists. Introducing a third scoring option (i.e. partially provided), however introduces subjectivity within the ratings. Despite our training of the raters, this was not sufficient to overcome the subjective dissimilarities. Therefore, results from the audio recordings should be interpreted with caution.

In summary, this pilot study demonstrates that an integrated biopsychosocial intervention is feasible and potentially effective in reducing functional disability in patients with CLBP who experience moderate complex psychosocial complaints. The study can act as a preliminary study for high-quality clinical trials in which the cost-effectiveness of an integrated biopsychosocial primary care intervention will be compared with regular secondary care interventions in patients with CLBP.

\section{ACKNOWLEDGEMENTS}

First, we would like to thank Marion de Mooij for her support and assistance during study. We acknowledge Paul Willems, Frans Abbink and Marlies den Hollander for their help in the development of the Back on Track intervention, and thank all participating patients for receiving the intervention and to express their experiences. Thanks to the participating physiotherapists, especially those who delivered the Back on Track intervention, audio recorded therapy sessions, and participated in the focus groups (Tom Hameleers, Frans Abbink, Remco Reijnders and Germaine Neumann). We would like to thank the Department of Rehabilitation in medicine MUMC +, Spine Centre MUMC + and Fy'net Collaboration for facilitating the delivery of the Back on Track intervention, as well as physicians (Marieke van Beugen and Robin Strackke) for delivering the Back on Track consultations and participating in the focus groups. Many thanks to Celine Kieftenburg and Jana Naumann who rated the audio recordings, and Arjan Kooistra who conducted the focus groups and the related analysis. Also we would like to thank Ton Ambergen for his help performing the statistical analyses, and to interpret and report these. Finally, we would like to thank Adelante (Centre of Expertise in Rehabilitation and Audiology), the Province of Limburg, and CZ Foundation for sponsoring the study. 


\section{REFERENCES}

1. Kamper SJ, Apeldoorn AT, Chiarotto A, et al. Multidisciplinary biopsychosocial rehabilitation for chronic low back pain. The Cochrane database of systematic reviews. 2014;9:CD000963.

2. Scerri M, de Goumoens P, Fritsch C, Van Melle G, Stiefel F, So A. The INTERMED questionnaire for predicting return to work after a multidisciplinary rehabilitation program for chronic low back pain. Joint Bone Spine. 2006;73(6):736-741.

3. George SZ, Fritz JM, Bialosky JE, Donald DA. The effect of a fear-avoidance-based physical therapy intervention for patients with acute low back pain: results of a randomized clinical trial. Spine. 2003;28(23):2551-2560.

4. Boonstra AM, Bühring M, Brouwers M, Bosma F, Schiphorst Preuper R. Patiënten met chronische pijnklachten op het grensvlak van revalidatiegeneeskunde en psychiatrie. Ned Tijdschr Pijn bestr. 2008;27(34):5-9.

5. Van Eijndhoven M, Gaasbeek Janzen M, Latta J, De Wit J. Rapport Medisch-specialistische revalidatie: zorg zoals revalidatieartsen plegen te bieden. Zorginstituut Nederland;2015.

6. Hoy D, Bain C, Williams G, et al. A systematic review of the global prevalence of low back pain. Arthritis and rheumatism. 2012;64(6):2028-2037.

7. Manchikanti L, Singh V, Falco FJ, Benyamin RM, Hirsch JA. Epidemiology of low back pain in adults. Neuromodulation. 2014;17 Suppl 2:3-10.

8. Onderhandelaarsresultaat medisch specialistische zorg 2014 t/m 2017. In. Den Haag: VWS; 2013.

9. Saunders RP, Evans MH, Joshi P. Developing a process-evaluation plan for assessing health promotion program implementation: a how-to guide. Health promotion practice. 2005;6(2):134-147.

10. Leeuw M, Goossens ME, van Breukelen GJ, et al. Exposure in vivo versus operant graded activity in chronic low back pain patients: results of a randomized controlled trial. Pain. 2008;138(1):192-207.

11. Smeets RJ, Vlaeyen JW, Hidding A, Kester AD, van der Heijden GJ, Knottnerus JA. Chronic low back pain: physical training, graded activity with problem solving training, or both? The one-year post-treatment results of a randomized controlled trial. Pain. 2008;134(3):263-276.

12. van Erp RMA, Huijnen IPJ, Koke AJA, Abbink FE, den Hollander M, Smeets R. Development and content of the biopsychosocial primary care intervention 'Back on Track' for a subgroup of people with chronic low back pain. Physiotherapy. 2017;103(2):160-166.

13. Mertens VC, Moser A, Verbunt J, Smeets R, Goossens M. Content Validity of the Credibility and Expectancy Questionnaire in a Pain Rehabilitation Setting. Pain practice : the official journal of World Institute of Pain. 2016.

14. Smeets RJ, Beelen S, Goossens ME, Schouten EG, Knottnerus JA, Vlaeyen JW. Treatment expectancy and credibility are associated with the outcome of both physical and cognitive-behavioral treatment in chronic low back pain. The Clinical journal of pain. 2008;24(4):305-315.

15. Leeuw M, Goossens ME, de Vet HC, Vlaeyen JW. The fidelity of treatment delivery can be assessed in treatment outcome studies: a successful illustration from behavioral medicine. Journal of clinical epidemiology. 2009;62(1):81-90.

16. De Vet HCW, Terwee CB, Mokkink LB, Knol DL. Measurement in Medicine: a practical guide. Cambridge: Cambridge University Press; 2011. 
17. Schoppink LE, van Tulder MW, Koes BW, Beurskens SA, de Bie RA. Reliability and validity of the Dutch adaptation of the Quebec Back Pain Disability Scale. Physical therapy. 1996;76(3):268-275.

18. Kopec JA, Esdaile JM, Abrahamowicz M, et al. The Quebec Back Pain Disability Scale. Measurement properties. Spine. 1995;20(3):341-352.

19. Smeets R, Koke A, Lin CW, Ferreira M, Demoulin C. Measures of function in low back pain/disorders: Low Back Pain Rating Scale (LBPRS), Oswestry Disability Index (ODI), Progressive Isoinertial Lifting Evaluation (PILE), Quebec Back Pain Disability Scale (QBPDS), and Roland-Morris Disability Questionnaire (RDQ). Arthritis care \& research. 2011;63 Suppl 11:S158-173.

20. Leeuw M, Goossens ME, van Breukelen GJ, et al. Measuring perceived harmfulness of physical activities in patients with chronic low back pain: the Photograph Series of Daily Activities — short electronic version. J Pain. 2007 Nov;8(11):840-9. doi: 10.1016/j.jpain.2007.05.013. PubMed PMID: 17632038

21. Vlaeyen JW, de Jong J, Geilen M, Heuts PH, van Breukelen G. Graded exposure in vivo in the treatment of pain-related fear: a replicated single-case experimental design in four patients with chronic low back pain. Behaviour research and therapy. 2001;39(2):151-166.

22. Lamb SE, Hansen Z, Lall R, et al. Group cognitive behavioural treatment for low-back pain in primary care: a randomised controlled trial and cost-effectiveness analysis. Lancet. 2010;375(9718):916-923.

23. Hall A, Richmond H, Copsey B, et al. Physiotherapist-delivered cognitive-behavioural interventions are effective for low back pain, but can they be replicated in clinical practice? A systematic review. Disability and rehabilitation. 2016:1-9.

24. Van Erp RMA, Huijnen IPJ, Jakobs MLG, Kleijnen J, Smeets RJEM. Effectiveness of primary care interventions using a biopsychosocial approach in chronic low back pain: a systematic review. Submitted.

25. Critchley DJ, Ratcliffe J, Noonan S, Jones RH, Hurley MV. Effectiveness and cost-effectiveness of three types of physiotherapy used to reduce chronic low back pain disability: a pragmatic randomized trial with economic evaluation. Spine. 2007;32(14):1474-1481.

26. Carnes D, Homer KE, Miles CL, et al. Effective delivery styles and content for self-management interventions for chronic musculoskeletal pain: a systematic literature review. The Clinical journal of pain. 2012;28(4):344-354.

27. Nijs J, Paul van Wilgen C, Van Oosterwijck J, van Ittersum M, Meeus M. How to explain central sensitization to patients with 'unexplained' chronic musculoskeletal pain: practice guidelines. Manual therapy. 2011;16(5):413-418.

28. Louw A, Zimney K, Puentedura EJ, Diener I. The efficacy of pain neuroscience education on musculoskeletal pain: A systematic review of the literature. Physiother Theory Pract. 2016;32(5):332-355.

29. Bishop A, Foster NE, Thomas E, Hay EM. How does the self-reported clinical management of patients with low back pain relate to the attitudes and beliefs of health care practitioners? A survey of UK general practitioners and physiotherapists. Pain. 2008;135(1-2):187-195.

30. Darlow B, Fullen BM, Dean S, Hurley DA, Baxter GD, Dowell A. The association between health care professional attitudes and beliefs and the attitudes and beliefs, clinical management, and outcomes of patients with low back pain: a systematic review. European journal of pain. 2012;16(1):3-17. 


\section{Chapter 7}

Spinal surgeons' opinions on preand postoperative rehabilitation in patients undergoing lumbar spinal fusion surgery: a survey-based study in the Netherlands and Sweden 



\section{ABSTRACT}

Study Design: A cross-sectional survey in the Netherlands and Sweden.

Objective: To investigate Dutch and Swedish spinal surgeons' opinions on spinal fusion pre- and postoperative rehabilitation.

Summary of Background Data: Lumbar spinal fusion surgery is increasingly provided in patients with chronic low back pain. No guidelines however exist for pre- and postoperative rehabilitation and it is unknown what opinions spinal surgeons currently have about pre- and postoperative rehabilitation.

Methods: A survey was circulated to Dutch and Swedish spinal surgeons. Reminders were sent after 4 and 8/9 weeks. Data of completed questionnaires of orthopaedic- and neurosurgeons currently performing lumbar spinal fusion were included for analysis. Analysis comprised a range of descriptive summaries (numerical, graphical, and tabular).

Results: Surveys of 34 Dutch and 48 Swedish surgeons were analysed. Surgeons provided preoperative information on postoperative mobilization. Spinal fusion techniques varied, but technique did not influence postoperative treatment. Swedish surgeons recommended slightly faster mobilization than Dutch (direct vs. 1-day postoperative), and more activities the first day (sitting, standing, walking). Stair climbing was the most reported discharge criterion; however, time point to start varied. More Swedish surgeons referred to postoperative physiotherapy than Dutch (88\% vs. 44\%). Time-point to start home activities varied from 1 week to > 6 months. Pain increase was allowed for < 24h (NL 81\%, SE 92\%).

Conclusions: Findings reflect variability in lumbar spinal fusion rehabilitation in two European countries, especially in postoperative phase. The study proposes many new research topics and acts as starting point for future research valuable for the spinal community.

\section{KEYWORDS}

Lumbar spinal fusion, fusion, spinal surgery, surgeon opinions, surgeon practice, rehabilitation, physiotherapy, low back pain, chronic low back pain, international survey 


\section{INTRODUCTION}

In patients with low back pain, initial management includes advice to stay active and/or conservative treatment.' When conservative treatments do not suffice and symptoms can be (partially) attributed to specific pathology (e.g. spinal stenosis, spondylolisthesis or degenerative disc disease (DDD)), invasive treatments like injections or surgical interventions are potential alternatives., ${ }^{2,3}$ Spinal fusion is one such surgical intervention that is commonly performed. Although its effectiveness and success rate has been criticized as conservative treatments (with lower burden) show equal outcomes, ${ }^{4}$ the incidence of lumbar spinal fusion is large and has increased worldwide. ${ }^{3,5,6}$ In the United States for example, the overall annual number of spinal fusion surgeries increased from 174,223 to 413,171 between 1998 and 2008. ${ }^{5}$ In Sweden, spinal fusion is also the most commonly performed surgery for spondylolisthesis and DDD. ${ }^{3}$

The major goal of fusion surgery is to reduce pain, increase function and health related quality of life. It has been demonstrated that preoperative fitness improves surgical outcome postoperatively. Hence, prehabilitation (i.e. preliminary rehabilitation prior to surgery) has become a growing field in spinal surgery. ${ }^{8-10}$ Evidence based guidelines for prehabilitation, however, do not exist. Similar is true for postoperative rehabilitation. As a consequence, variability in practice is seen between spinal surgeons at pre- and postoperative phase (i.e. variability in discharge criteria, outcome measures, hospital stay, follow-up frequency and intensity). ${ }^{11}$ Also from clinical experience we notice uncertainty among health care specialists about appropriate advice for mobilization, loading, functional activities or rehabilitation after spinal fusion. It is possible that this fuels uncertainty in some patients, leading to fear of movement and increased disability.

Since there is uncertainty and variability in practice, it is important to investigate what kind of opinions spinal surgeons have about pre- and postoperative rehabilitation. Improving understanding about current opinions is valuable when developing consensus guidelines and to stimulate clinical trials to evaluate different strategies. To our knowledge, no study has yet been performed to investigate spinal surgeons' opinions in the Netherlands and Sweden. The purpose of the current study was to create an inventory of the opinions of Dutch and Swedish spinal surgeons regarding pre-and postoperative rehabilitation for patients with lumbar DDD who undergo spinal fusion.

\section{MATERIALS AND METHODS}

\section{DESIGN AND POPULATION}

A cross-sectional survey was conducted among Dutch and Swedish spinal surgeons. The Dutch Medical Ethics Approval Committee approved the Dutch survey (METC 14-5-035). According to the Swedish Law, this study did not fall under the Act (2003:460) concerning the Ethical Review of Research Involving Humans. The study is reported according to The Strengthening the Reporting of Observational Studies in Epidemiology (STROBE) statement. ${ }^{12}$ 
Recruitment was conducted via the Dutch Spine Society (DSS) and Swedish Society of Spinal Surgeons (4S). Members of the DSS and S4 were sent an invitation letter for participation and a link to an online survey distributed by MEMIC (Centre for Data and Information Management, NL; 11/2014-01/2015) or Webropol online survey tool (SE; 05/2015-09/2015). By returning the questionnaire, surgeons gave written consent for participation. Reminders were sent out after 4 and 8 weeks (NL), and 4 and 9 weeks (SE).

\section{SURVEY}

The survey was developed by a multi-professional team; a spinal surgeon (PW), a consultant in rehabilitation medicine (RS), a physiotherapist (ML), and a resident in rehabilitation medicine and orthopaedics (JJ). Questions were originally formulated in English and sent to Dutch spinal surgeons. The survey consisted mainly of closed questions, subdivided into five parts: general information, pre-operative phase, operative phase, postoperative phase and follow-up (outpatient) phase. The English questionnaire was translated into Swedish and sent to Swedish spinal surgeons. Some answering options were slightly adapted based on recommendations from the 4S (Appendix 1).

\section{DATA COLLECTION}

MEMIC (NL) and Webropol (SE) distributed the survey, collected data and provided Dutch and Swedish researchers with completed data. Researchers in both countries were responsible for data cleaning their respective surveys. An independent Swedish researcher merged the datasets.

\section{DATA ANALYSIS}

Data analysis was performed in the Netherlands using IBM SPSS Statistics 22. Only data from orthopaedic surgeons and neurosurgeons currently performing lumbar spinal fusion were included for analysis. Incomplete questionnaires and data of retired surgeons or surgeons working in countries other than the Netherlands or Sweden were excluded. Analysis comprised a range of descriptive summaries (numerical, graphical, and tabular).

\section{RESULTS}

Forty of the 105 Dutch spinal surgeons returned the questionnaire (response rate 38\%; Figure 1). Six provided incomplete data, resulting in 34 surveys analysed. In Sweden, 48 of the 140 spinal surgeons returned the questionnaire (response rate 34\%). All were included for analysis. 
Figure 1 Flowchart of responders

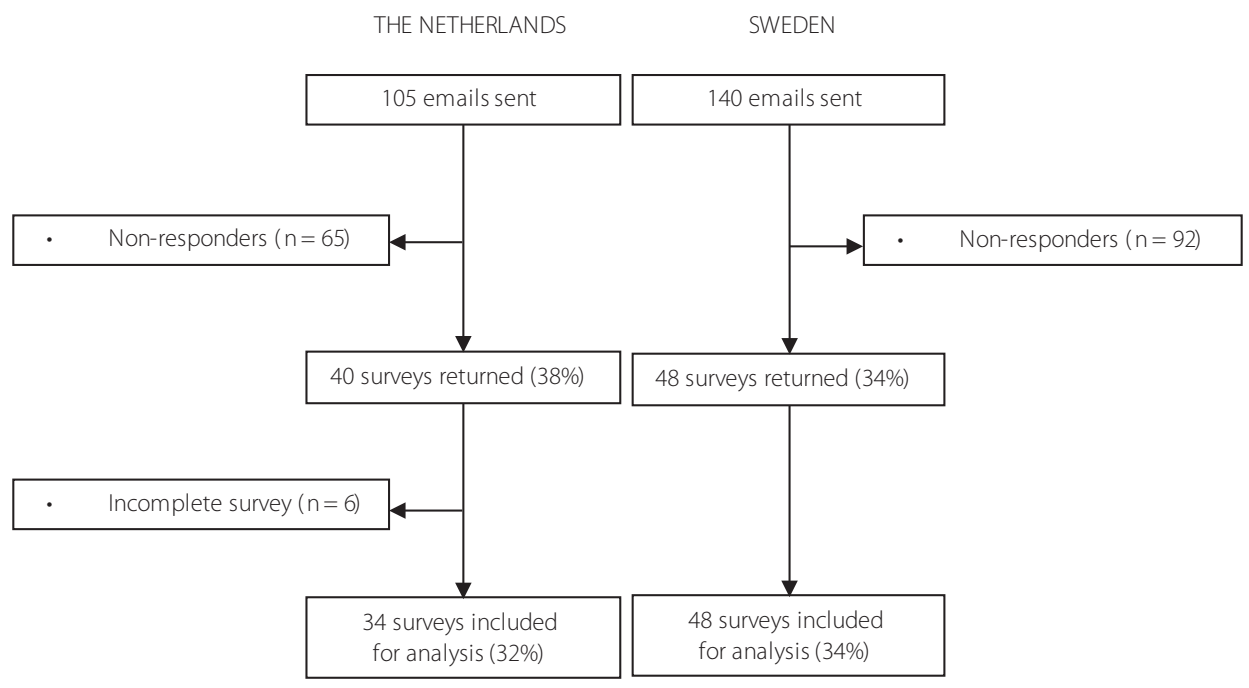

\section{CHARACTERISTICS OF SPINAL SURGEONS}

Most spinal surgeons worked as orthopaedic surgeons (NL 71\%, SE 94\%; Table 1). Approximately half of all Dutch spinal surgeons and three-quarter of all Swedish spinal surgeons had experience for $>10$ years. The number of spinal fusions performed per year varied considerably in both countries. Years of experience and number of fusions per year did not influence pre- and postoperative treatment. 
Table 1 Characteristics of Dutch responders $(n=34)$ and Swedish responders $(n=48)$

\begin{tabular}{|c|c|c|c|c|c|}
\hline & \multicolumn{2}{|c|}{ The Netherlands } & \multicolumn{3}{|c|}{ Sweden } \\
\hline & $\begin{array}{c}\text { Orthopedic } \\
\text { surgeons } \\
\text { n (\%) }\end{array}$ & $\begin{array}{c}\text { Neurosurgeons } \\
n(\%)\end{array}$ & $\begin{array}{c}\text { Orthopedic } \\
\text { surgeons } \\
\text { n (\%) }\end{array}$ & $\begin{array}{c}\text { Neurosurgeons } \\
n(\%)\end{array}$ & $\begin{array}{l}\text { Both* } \\
n(\%)\end{array}$ \\
\hline No. of responders & 24 & 10 & 45 & 2 & 1 \\
\hline \multicolumn{6}{|l|}{ Clinical experience } \\
\hline$<5$ years & $6(25)$ & $0(0)$ & $3(7)$ & $0(0)$ & $0(0)$ \\
\hline $5-10$ years & $6(25)$ & $4(40)$ & $8(18)$ & $0(0)$ & $0(0)$ \\
\hline$>10$ years & $12(50)$ & $6(60)$ & $34(76)$ & $2(100)$ & $1(100)$ \\
\hline \multicolumn{6}{|c|}{ No. of fusions per year } \\
\hline $1-25$ & $12(50)$ & $1(10)$ & $20(44)$ & $0(0)$ & $0(0)$ \\
\hline $26-50$ & $2(8)$ & $3(30)$ & $14(31)$ & $0(0)$ & $0(0)$ \\
\hline $51-75$ & $7(29)$ & $3(30)$ & $7(16)$ & $0(0)$ & $0(0)$ \\
\hline $76-100$ & $2(8)$ & $1(10)$ & $2(4)$ & $1(50)$ & $0(0)$ \\
\hline$>100$ & $1(4)$ & $2(20)$ & $2(4)$ & $1(50)$ & $1(100)$ \\
\hline
\end{tabular}

\section{PRE-OPERATIVE PHASE}

Most Dutch and Swedish spinal surgeons provided preoperative information on postoperative mobilisation (NL 97\%, SE 98\%). Preoperative information was mainly provided by surgeons themselves (NL 100\%, SE 96\%). Other less frequently reported pathways were by nurse (NL 38\%, SE 23\%), letter (NL 44\%, SE 19\%), physiotherapist (NL 9\%, SE 54\%), brochure (NL 12\%, SE 10\%), website (NL 6\%) and/or occupational therapist (SE 4\%). Referral to preoperative physiotherapy varied, but was recommended by (almost) one-third of the Dutch (27\%) and Swedish surgeons (35\%). Other surgeons referred only rarely/sometimes (NL 35\%, SE 46\%) or not at all (NL 38\%, SE 19\%).

\section{OPERATIVE PHASE}

Both countries used multiple spinal fusion techniques, but most Dutch (91\%) and almost three-quarter of the Swedish spinal surgeons (73\%) reported that the technique, regardless of their preference, did not influence postoperative treatment.

\section{POSTOPERATIVE PHASE}

The majority of the Dutch spinal surgeons recommended mobilisation the first day postoperative (63\%), while most Swedish spinal surgeons recommended mobilisation directly (73\%; Figure 2). 
Figure 2 Time points at which patients can be mobilised after lumbar spinal fusion surgery

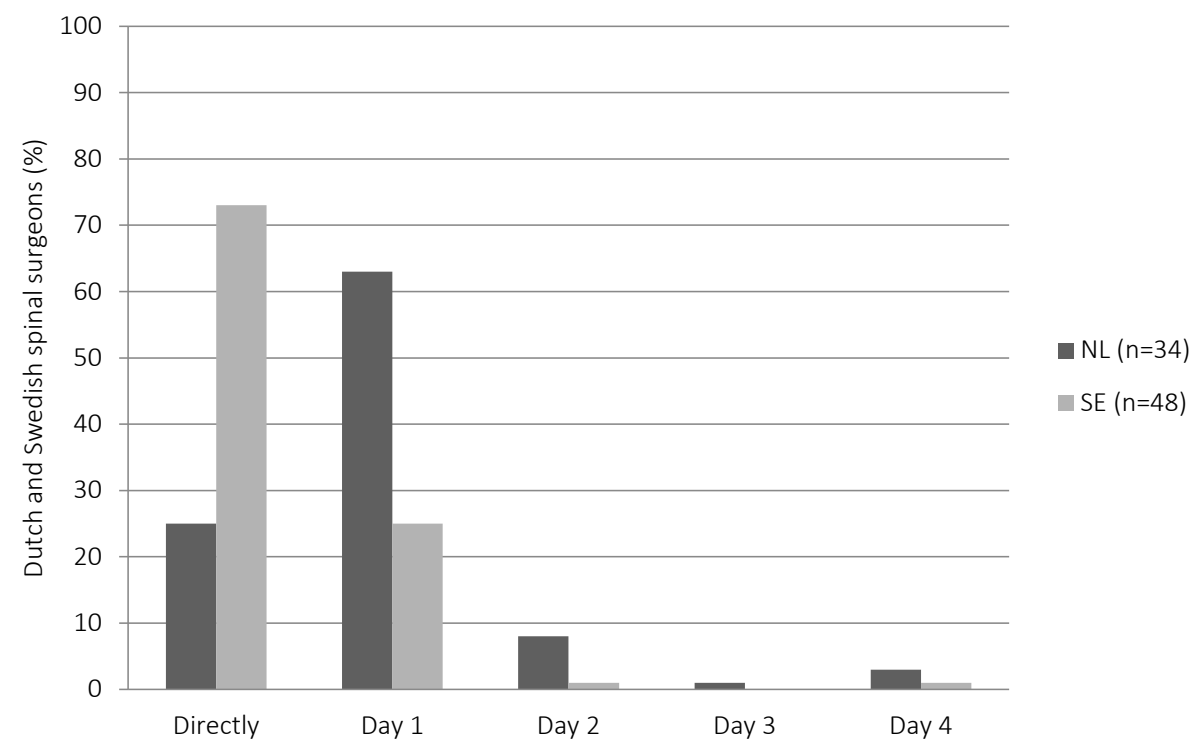

Almost all Dutch and Swedish spinal surgeons advised mobilisation to be guided by a physiotherapist (NL 97\%, SE 100\%). At the first day postoperatively, the majority of the Dutch and Swedish spinal surgeons recommended sitting in bed (88\% and 98\%; Figure 3) while most Swedish spinal surgeons also advised standing (98\%), and walking with support (85\%). The ability to climb stairs was the most reported physical discharge criterion (NL 74\%, SE 56\%). 
Figure 3 Time points at which functional activities can be performed postoperatively as reported by Dutch (above; $n=34$ ) and Swedish (below; $n=48$ ) spinal surgeons

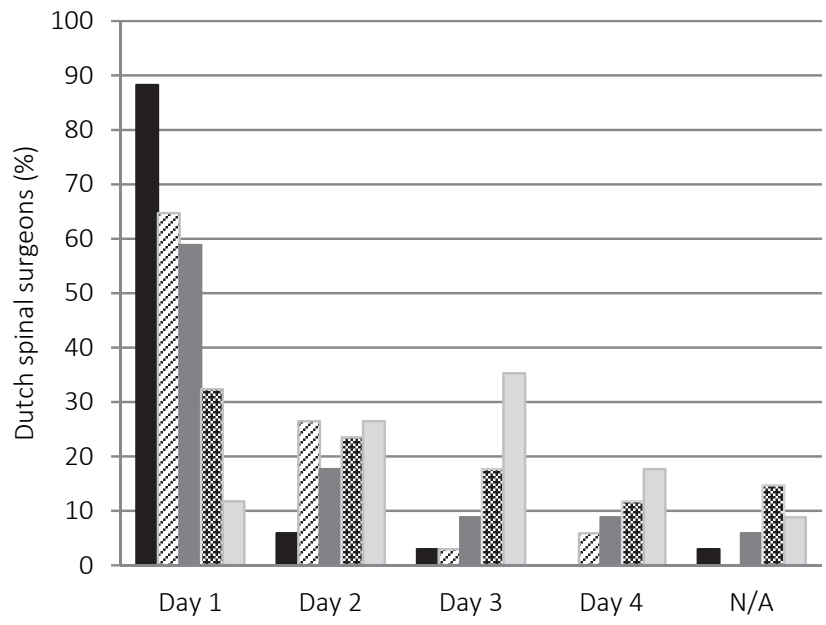

\section{Sitting in bed} $\square$ Standing

Walking with support

Walking without support

$\square$ Climbing stairs

Sitting in bed $\triangle$ Standing

Walking with support

Walking without support

$\square$ Climbing stairs

Most Dutch and Swedish spinal surgeons recommended supervision while start standing (94\% vs. $85 \%$ ), walking with support ( $97 \%$ vs. $88 \%$ ), walking without support ( $86 \%$ vs. $92 \%$ ), and stair climbing (100\% vs. 98\%). Dutch spinal surgeons also recommended supervision while start sitting in bed (76\%). 
Almost all Dutch and Swedish spinal surgeons agreed that pain after mobilisation was permitted (NL94\%, SE 98\%). Approximately one third of the Dutch (28\%) and two third of the Swedish spinal surgeons (66\%) reported that this pain was permitted for maximally 6 hours. Half (53\%) of the Dutch, and 26\% of the Swedish spinal surgeons permitted increased pain until 24 hours. Remaining surgeons permitted pain over 24 hours.

\section{POSTOPERATIVE OUTPATIENT PHASE}

Almost all Dutch and Swedish spinal surgeons advised walking and stair climbing in the first week (Table $2 \mathrm{a}$ and $2 \mathrm{~b}$ ). There was no consensus on when to return to other activities as advice varied considerably from the first day postoperatively to $>6$ months. A few surgeons discouraged running, rotating, extending and jumping.

Table 2a Time points at which activities are allowed to be performed as reported by Dutch spinal surgeons $(n=34)$

\begin{tabular}{|c|c|c|c|c|c|c|c|}
\hline & \multicolumn{7}{|c|}{$\begin{array}{l}\text { The Netherlands } \\
\text { No. surgeons (\%) }\end{array}$} \\
\hline & $\begin{array}{l}1-7 \\
\text { days }\end{array}$ & $\begin{array}{c}1-4 \\
\text { weeks }\end{array}$ & $\begin{array}{c}5-8 \\
\text { weeks }\end{array}$ & $\begin{array}{c}9-12 \\
\text { weeks }\end{array}$ & $\begin{array}{c}3-6 \\
\text { months }\end{array}$ & $\begin{array}{l}>6 \\
\text { months }\end{array}$ & Never \\
\hline Walking & $34(100)$ & 0 & 0 & 0 & 0 & 0 & 0 \\
\hline Stair climbing & $31(91)$ & $2(6)$ & $1(3)$ & 0 & 0 & 0 & 0 \\
\hline Running & $2(6)$ & $2(6)$ & $6(18)$ & $3(9)$ & $14(41)$ & $6(18)$ & $1(3)$ \\
\hline Bending forward & $6(18)$ & $4(12)$ & $10(29)$ & $4(12)$ & $10(29)$ & 0 & 0 \\
\hline $\begin{array}{l}\text { Rotation to end } \\
\text { position }\end{array}$ & $6(18)$ & $3(9)$ & $6(18)$ & $8(24)$ & $11(32)$ & 0 & 0 \\
\hline $\begin{array}{l}\text { Extension to } \\
\text { end position }\end{array}$ & $6(18)$ & $2(6)$ & $7(21)$ & $9(26)$ & $10(29)$ & 0 & 0 \\
\hline Cycling & $2(6)$ & $3(9)$ & $18(53)$ & $3(9)$ & $7(21)$ & $1(3)$ & 0 \\
\hline Driving car & $2(6)$ & $7(21)$ & $20(59)$ & $3(9)$ & $2(6)$ & 0 & 0 \\
\hline $\begin{array}{l}\text { Swimming } \\
\text { (breaststroke) }\end{array}$ & $1(3)$ & $3(9)$ & $12(35)$ & $7(21)$ & $10(29)$ & $1(3)$ & 0 \\
\hline Lifting (2.5 kg) & $3(9)$ & $2(6)$ & $9(26)$ & $6(18)$ & $9(26)$ & $5(15)$ & 0 \\
\hline Jumping $(10 \mathrm{~cm})$ & $2(6)$ & 0 & $5(15)$ & $5(15)$ & $15(44)$ & $6(18)$ & $1(3)$ \\
\hline
\end{tabular}


Table $\mathbf{2} \mathbf{b}$ Time points at which activities are allowed to be performed as reported by Swedish spinal surgeons $(n=48)$

\begin{tabular}{|c|c|c|c|c|c|c|c|}
\hline & \multicolumn{7}{|c|}{$\begin{array}{c}\text { Sweden } \\
\text { No. surgeons (\%) }\end{array}$} \\
\hline & $\begin{array}{l}1-7 \\
\text { days }\end{array}$ & $\begin{array}{c}1-4 \\
\text { weeks }\end{array}$ & $\begin{array}{c}4-8 \\
\text { weeks }\end{array}$ & $\begin{array}{c}8-12 \\
\text { weeks }\end{array}$ & $\begin{array}{c}3-6 \\
\text { months }\end{array}$ & $\begin{array}{c}>6 \\
\text { months }\end{array}$ & Never \\
\hline Walking & $48(100)$ & 0 & 0 & 0 & 0 & 0 & 0 \\
\hline Stair climbing & $46(96)$ & $2(4)$ & 0 & 0 & 0 & 0 & 0 \\
\hline Running & $2(4)$ & $3(6)$ & $10(21)$ & $8(17)$ & $19(40)$ & $4(8)$ & $2(4)$ \\
\hline Bending forward & $17(35)$ & $13(27)$ & $8(17)$ & $4(8)$ & $6(13)$ & 0 & 0 \\
\hline $\begin{array}{l}\text { Rotation to end } \\
\text { position }\end{array}$ & $12(25)$ & $12(25)$ & $6(13)$ & $4(8)$ & $10(21)$ & $2(4)$ & $2(4)$ \\
\hline $\begin{array}{l}\text { Extension to } \\
\text { end position }\end{array}$ & $15(31)$ & $10(21)$ & $8(17)$ & $5(10)$ & $7(15)$ & $1(2)$ & $2(4)$ \\
\hline Cycling & $3(6)$ & $14(29)$ & $11(23)$ & $8(17)$ & $10(21)$ & $2(4)$ & 0 \\
\hline Driving car & $2(4)$ & $18(38)$ & $12(25)$ & $12(25)$ & $3(6)$ & $1(2)$ & 0 \\
\hline $\begin{array}{l}\text { Swimming } \\
\text { (breaststroke) }\end{array}$ & $2(4)$ & $7(15)$ & $19(40)$ & $8(17)$ & $10(21)$ & $2(4)$ & 0 \\
\hline Lifting (2.5 kg) & $7(15)$ & $7(15)$ & $12(25)$ & $7(15)$ & $11(23)$ & $4(8)$ & 0 \\
\hline Jumping $(10 \mathrm{~cm})$ & $2(4)$ & $5(10)$ & $6(13)$ & $9(19)$ & $18(38)$ & $5(10)$ & $3(6)$ \\
\hline
\end{tabular}

Advice on maximum lifting weight and jumping height varied in both countries from $<5$ kilograms or $<5$ centimetres to no limit at all (Figure 4 and 5). Additional Swedish recommendations for lifting included; patient-dependent, not applicable if proper lifting techniques are used, and allowed until patient's pain threshold. For jumping, one additional recommendation was reported; patient's choice. Five Swedish spinal surgeons had no opinion and thirteen did not report maximum lifting weight. 
Figure 4 Maximum lifting weight (kilograms) as advised by Dutch and Swedish spinal surgeons

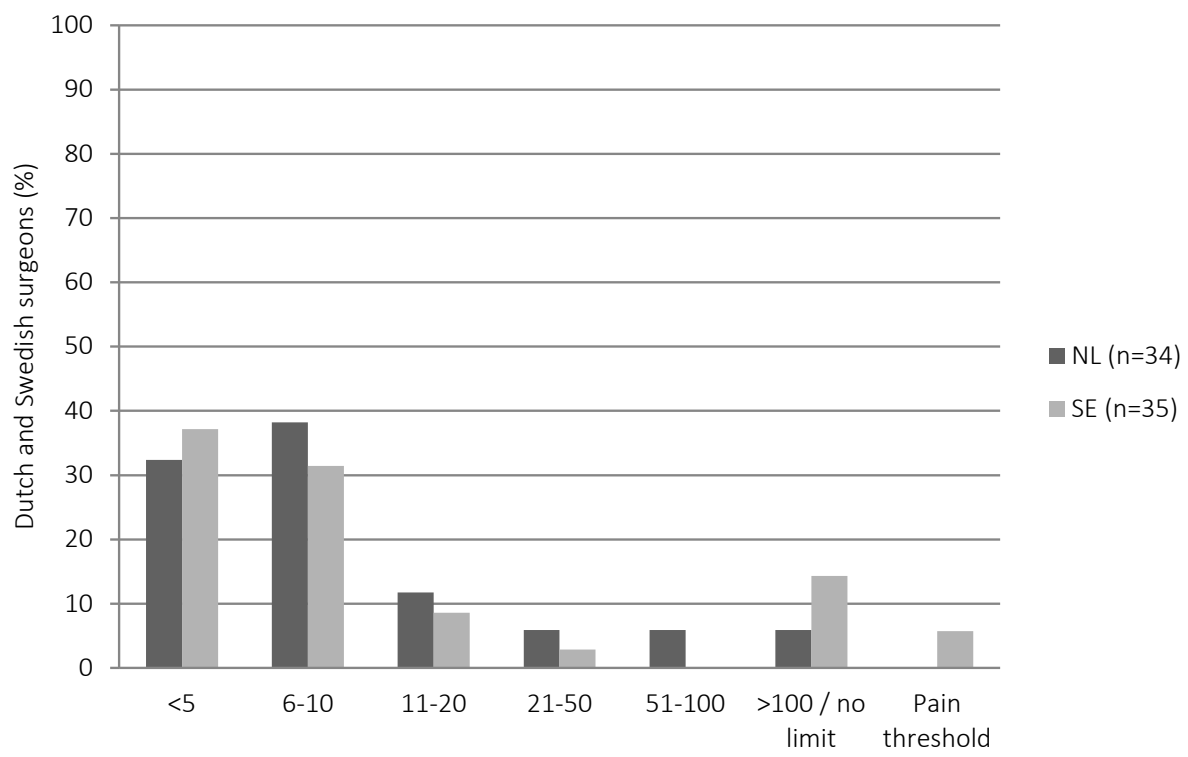

Figure 5 Maximum jumping height (centimetres) as advised by Dutch and Swedish spinal surgeons

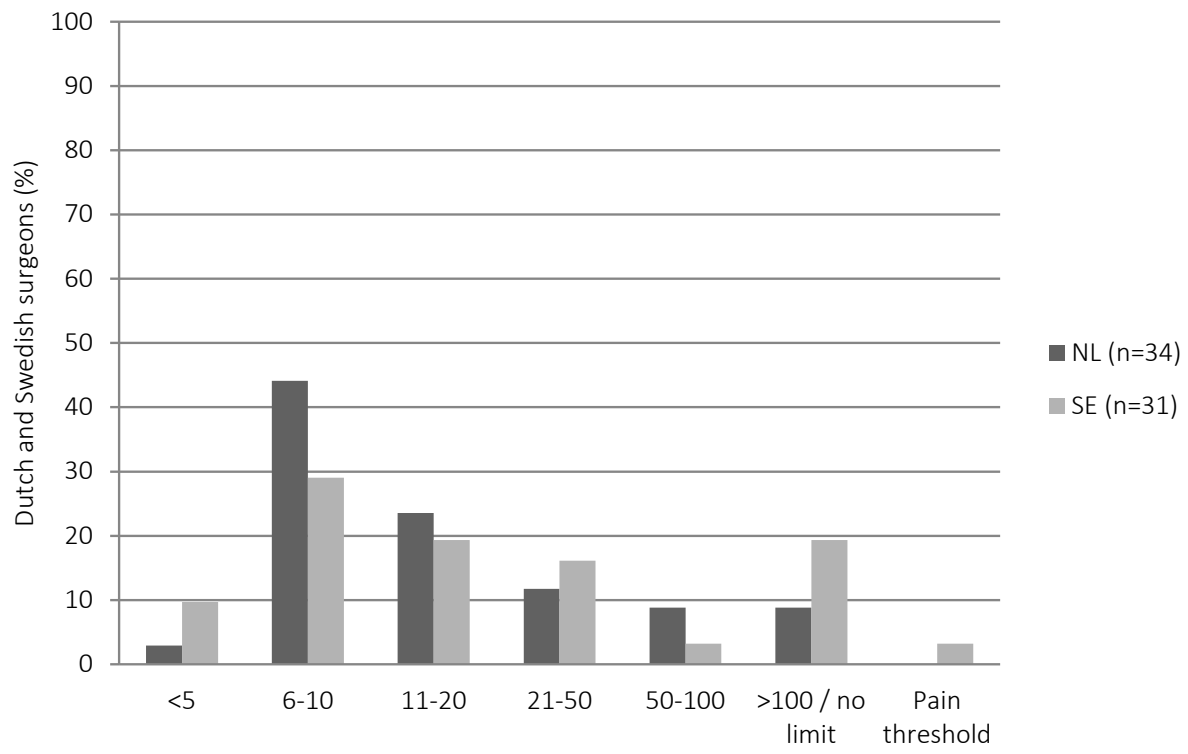


More than two-third of the Dutch and Swedish spinal surgeons advised against wearing a corset (68\% and 69\%). The small group recommending a corset, showed large variation in wearing advice (e.g. until 6 weeks, until 3 months, patient's choice, during lifting or physical activities).

Fewer Dutch (44\%) than Swedish spinal surgeons (88\%) referred to postoperative physiotherapy. Spinal surgeons who did prescribe physiotherapy varied in advice when to start physiotherapy; ranging from immediately to 9-12 weeks. Top three treatments that should not be provided were (1) manual therapy (NL 82\%, SE 58\%), (2) mechanical diagnosis therapy (McKenzie; NL 35\%, SE 52\%) and; (3) sensory stimulation (massage) (NL 32\%, SE 38\%). Of the Swedish spinal surgeons, $27 \%$ had no opinion. Also one Dutch spinal surgeon reported to have no idea.

Advice for return to work was most commonly reported to be dependent on type of work. Other less frequently reported approaches were; a pain-contingent increase, and time-contingent increase in working hours. One Dutch and one Swedish spinal surgeon had no opinion. Other factors reported by Swedish surgeons were 'based on duration of disorder', 'psychological well-being,',radiologic healing,' 'whether occupation can be adapted to a high degree', and 'never before 3 months'.

\section{DISCUSSION}

This study provides an overview of opinions of Dutch and Swedish spinal surgeons about current pre- and postoperative rehabilitation in patients undergoing lumbar spinal fusion. One interesting findings was that nearly all surgeons provided preoperative information on postoperative mobilisation. This is comparable to British spinal surgeons of whom 91\% provided preoperative information. ${ }^{11}$ In the Netherlands, additional preoperative information was most frequently provided by a letter and/or nurse, while in Sweden this was done by a physiotherapist. Although different professionals were involved, findings indicate consensus on providing preoperative information. Further studies are required to investigate what the content of information should be, who should deliver it and at what time point.

Consensus on preoperative information links nicely with the growing evidence for prehabilitation. ${ }^{713,14}$ Recent literature supports referral to prehabilitation programmes as it facilitates mobilisation and lowers the length of hospital stay as compared to standard care. ${ }^{8}$ Moreover, if cognitive behavioural elements are added, patients show a lower intake of analgesics. ${ }^{9}$ Referral to prehabilitation was however not consistently recommended in both countries. One possible explanation is that prehabilitation is still in its infancy, not well known by spinal surgeons, and not structurally implemented in clinical practice.

Swedish spinal surgeons recommended slightly faster postoperative mobilisation in the hospital than Dutch spinal surgeons, but overall, both countries mobilised patients within one day. This corresponds with current physiotherapy practice in the UK where most patients were seen within the first day postoperatively. ${ }^{15}$ Swedish spinal surgeons started activities like standing and walking slightly earlier than Dutch spinal surgeons. This might presume faster discharge and therefore shorter duration of hospital stay in Sweden although this assumption could not be checked with this study. This study identified that both countries used the same physical discharge criteria (i.e. the ability to climb stairs independently). The time point to start this activity 
varied in both countries from day 1 to 4 . It is therefore possible that the duration of hospital stay varies within each country. It is recommended to find consensus on the time point to start such functional activities as it will likely improve uniformity on discharge and shorten hospital stay.

In our study, few surgeons advised never to jump or run again, while others advised to jump or run in the first week. Furthermore, some surgeons allowed maximally $5 \mathrm{~cm}$ for jumping, or $5 \mathrm{~kg}$ for lifting, while others reported no limits at all. It seems that surgeons have varying ideas about the time point to start these activities and its intensity, or as few surgeons in our study reported, have no opinion at all. Variability in advice corresponds with Rushton et al. ${ }^{11}$ where recommendations for activities like jogging, sports, and lifting ranged from 2 weeks to 9 months. The finding that surgeons working in different European countries provide different advice, suggests the need for clinical guidance.

Another important finding was that pain is permitted as part of the rehabilitation process although it seemed hard to define at what time point the mobilisation strategy should be adjusted. The majority allowed an increase of pain for $<24$ hours. Of note is that more than half of the Swedish surgeons allowed pain for $<6$ hours. Further studies could investigate whether increased pain symptoms after activities should be used to modify rehabilitation.

The final major finding was the lack of consensus regarding postoperative referral to physiotherapy (except from Swedish surgeons), the time point to start postoperative physiotherapy, as well as the type of therapy. Postoperative physiotherapy seems beneficial for recovery ${ }^{16}$, but currently little evidence is available for optimal timing and specific treatment elements. Oestergaard et al. for example, found that initiation of rehabilitation at 6 weeks was less effective and more expensive than initiation of rehabilitation at 12 weeks. ${ }^{17,18}$ Additionally they reported that adding cognitive behavioural elements could increase the overall effectiveness. Abbot et al. ${ }^{19}$ provided early rehabilitation with cognitive behavioural elements (within the first three months) and showed significant larger improvements in functional disability, self-efficacy, outcome expectancy, and fear of movement/(re)injury than exercise therapy alone. This study highlights the potential role of cognitive behavioural elements and early postoperative rehabilitation.

The study included data of both Dutch and Swedish spinal surgeons and therefore provided an overview of surgeons' practice in two European countries. The number of surgeons analysed is comparable to other recently conducted cross-sectional studies. ${ }^{11,20}$ We discovered consistent opinions valuable for consensus guidelines, as well as inconsistent opinions and variability in practice leading to new research topics. We believe this study can act as a starting point for future research and will be valuable for the spinal community.

One limitation of the study is the relatively low response rate (38\% and 34\%) which increases the risk for bias. It is possible that surgeons with more interest in rehabilitation were more likely to complete the survey than surgeons with less interest. Consequently, opinions might have been different between responders and non-responders and therefore not entirely reflect clinical practice. 
There are methodological and process challenges associated with conducting a study across two countries. Multiple ethical committees and advisory boards must be considered with cultural variance and recommendations. One example for this study is the recommendation of the $4 \mathrm{~S}$ to slightly adapt the Swedish survey. Eventually these adjustments have not influenced our results since our aim was to describe the findings narratively.

It is recommended for future studies to systematically translate the survey and to keep questions and answering options standardised. Transparency, communication, structured management and documentation are key characteristics. It is recommended to adopt a study protocol and to appoint a project leader to systematically conduct an international study. For surgeons performing lumbar spinal fusion surgery it is desirable to provide clear and consistent instructions about the intensity and frequency of daily life activities (e.g. stair climbing, running and lifting), as well as the time point to start. Future trials are needed to investigate the time point to start preand post-rehabilitation, which health care professionals should be involved and what therapy elements are required for optimal recovery.

In summary, the findings of this study suggest variability in lumbar spinal fusion rehabilitation opinions in two European countries, especially in the postoperative phase. It seems unclear which activities are allowed, at what time point, and under what circumstances. Furthermore, physiotherapy (pre- and post-operative) seems to be less integrated in the rehabilitation process yet, although supported by literature. Future studies are needed to find consensus on suggested topics and to evaluate the effectiveness of treatment approaches to improve pre- and postoperative rehabilitation for patients undergoing spinal fusion.

\section{ACKNOWLEDGEMENTS}

We acknowledge the members of the Dutch Spine Society and the Swedish Society of Spinal Surgeons for their participation in this study. Furthermore, we would like to thank Max Jakobsson for his contribution to the merging of data and Ken Stewart for editing the manuscript. 


\section{REFERENCES}

1. Koes BW, van Tulder M, Lin CW, et al. An updated overview of clinical guidelines for the management of non-specific low back pain in primary care. European spine journal: official publication of the European Spine Society, the European Spinal Deformity Society, and the European Section of the Cervical Spine Research Society 2010;19:2075-94.

2. Jacobs WC, Rubinstein SM, Koes B, et al. Evidence for surgery in degenerative lumbar spine disorders. Best practice \& research. Clinical rheumatology 2013;27:673-84.

3. Stromqvist B, Fritzell P, Hagg O, et al. Swespine: the Swedish spine register : the 2012 report. European spine journal: official publication of the European Spine Society, the European Spinal Deformity Society, and the European Section of the Cervical Spine Research Society 2013;22:953-74.

4. Brox JI, Nygaard OP, Holm I, et al. Four-year follow-up of surgical versus non-surgical therapy for chronic low back pain. Annals of the rheumatic diseases 2010;69:1643-8.

5. Rajaee SS, Bae HW, Kanim LE, et al. Spinal fusion in the United States: analysis of trends from 1998 to 2008. Spine 2012;37:67-76.

6. Du Bois M, Szpalski M, Donceel P. A decade's experience in lumbar spine surgery in Belgium: sickness fund beneficiaries, 2000-2009. European spine journal: official publication of the European Spine Society, the European Spinal Deformity Society, and the European Section of the Cervical Spine Research Society 2012;21:2693-703.

7. Snowden CP, Prentis J, Jacques B, et al. Cardiorespiratory fitness predicts mortality and hospital length of stay after major elective surgery in older people. Ann Surg 2013;257:999-1004.

8. Nielsen PR, Jorgensen LD, Dahl B, et al. Prehabilitation and early rehabilitation after spinal surgery: randomized clinical trial. Clinical rehabilitation 2010;24:137-48.

9. Rolving N, Nielsen CV, Christensen FB, et al. Preoperative cognitive-behavioural intervention improves in-hospital mobilisation and analgesic use for lumbar spinal fusion patients. BMC musculoskeletal disorders 2016;17:217.

10. Lotzke H, Jakobsson M, Brisby H, et al. Use of the PREPARE (PREhabilitation, Physical Activity and exeRcisE) program to improve outcomes after lumbar fusion surgery for severe low back pain: a study protocol of a person-centred randomised controlled trial. BMC musculoskeletal disorders 2016;17:349.

11. Rushton A, White L, Heap A, et al. Evaluation of current surgeon practice for patients undergoing lumbar spinal fusion surgery in the United Kingdom. World J Orthop 2015;6:483-90.

12. von Elm E, Altman DG, Egger M, et al. The Strengthening the Reporting of Observational Studies in Epidemiology (STROBE) statement: guidelines for reporting observational studies. Lancet 2007;370:1453-7.

13. Santa Mina D, Clarke H, Ritvo P, et al. Effect of total-body prehabilitation on postoperative outcomes: a systematic review and meta-analysis. Physiotherapy 2014;100:196-207.

14. Minnella EM, Bousquet-Dion G, Awasthi R, et al. Multimodal prehabilitation improves functional capacity before and after colorectal surgery for cancer: a five-year research experience. Acta Oncol 2017;56:295-300.

15. Rushton A, Wright C, Heap A, et al. Survey of current physiotherapy practice for patients undergoing lumbar spinal fusion in the United Kingdom. Spine 2014;39:E1380-7.

16. McGregor AH, Probyn K, Cro S, et al. Rehabilitation following surgery for lumbar spinal stenosis. A Cochrane review. Spine 2014;39:1044-54. 
17. Oestergaard $L G$, Nielsen $C V$, Bunger $C E$, et al. The effect of early initiation of rehabilitation after lumbar spinal fusion: a randomized clinical study. Spine 2012;37:1803-9.

18. Oestergaard LG, Christensen FB, Nielsen CV, et al. Early versus late initiation of rehabilitation after lumbar spinal fusion: economic evaluation alongside a randomized controlled trial. Spine 2013;38:1979-85.

19. Abbott AD, Tyni-Lenne R, Hedlund R. Early rehabilitation targeting cognition, behavior, and motor function after lumbar fusion: a randomized controlled trial. Spine 2010;35:848-57.

20. Janssen ER, Scheijen EE, van Meeteren NL, et al. Determining clinical practice of expert physiotherapy for patients undergoing lumbar spinal fusion: a cross-sectional survey study. European spine journal : official publication of the European Spine Society, the European Spinal Deformity Society, and the European Section of the Cervical Spine Research Society 2016;25:1533-41. 


\section{APPENDIX}

\section{APPENDIX I DUTCH (NL) AND SWEDISH (SE) SURVEY}

\section{(7) Maastricht UMC+}

UNIVERSITY OF GOTHENBURG

\section{Introduction}

Dear colleague and spinal surgeon,

Lumbar spinal fusion is a commonly performed treatment for lumbar spondylolysis/-olisthesis or symptomatic degenerative disc disease. However, there is no consensus on the postoperative treatment strategy and rehabilitation in current literature.

In order to get more insight in the current clinical practice we ask you, as a spinal surgeon, to anonymously fill in a short questionnaire, which will take 5-10 minutes. Spinal surgeons in both The Netherlands and in Sweden are asked to participate. We need your experience in lumbar spinal fusion to achieve consensus in the future on the postoperative treatment strategy and rehabilitation.

\section{General}

1. What is your medical discipline? Multiple answers are possible (NL)

$\square \quad$ Orthopedics

$\square$ Neurosurgery

$\square$ Other:

What is your medical specialty? (SE)

$\square$ Orthopedics

$\square \quad$ Neurosurgery

$\square$ Both

$\square$ Other

If other, which one?

2. How many years of experience do you have as a spinal surgeon? (NL)

$$
\begin{aligned}
& \square \quad<5 \text { years } \\
& \square \quad 5-10 \text { years } \\
& \square>10 \text { years }
\end{aligned}
$$

How many years of experience do you have as a spinal surgeon? (SE)

$$
\begin{aligned}
& \square \quad<5 \text { years } \\
& \square \quad 5-10 \text { years } \\
& \square \quad>10 \text { years }
\end{aligned}
$$


3. How many lumbar spinal fusions do you perform per year? (NL)
$\square \quad 0$
$\square \quad 1-25$
$\square \quad 26-50$
$\square \quad 51-75$
$\square \quad 76-100$
$\square>100$

How many lumbar spinal fusions do you perform per year? (SE)
$\square \quad 0$
$\square \quad 1-25$
$\square \quad 26-50$
$\square \quad 51-75$
$\square \quad 76-100$
$\square>100$

\section{Preoperative Phase}

4. Do you provide information to the patient about postoperative mobilisation before surgery? (NL)
$\square$ Never
$\square$ Rarely
$\square$ Sometimes
$\square$ Often
$\square$ Always

Do you provide information to the patient about postoperative mobilisation before surgery? (SE)
$\square \quad$ No
$\square \quad$ Yes
$\square \quad$ Sometimes

5. How do patients get the information? (multiple options possible) (NL)
$\square$ by the doctor
$\square$ by the nurse
$\square \quad$ by letter
$\square$ by other, please specify how:

How do patients get the information? (SE)

$\square$ by the doctor

$\square$ by the nurse

$\square$ by letter

$\square$ by other, please specify by whom:

$\square \quad$ the patient doesn't get any information 
6. Do you refer your patients - scheduled for lumbar fusion surgery - for a preoperative programme guided by a physiotherapist? (NL)
$\square \quad$ Never
$\square$ Rarely
$\square \quad$ Sometimes
$\square \quad$ Often
$\square$ Always

Do you refer your patients - scheduled for lumbar fusion surgery - for a preoperative programme guided by a physiotherapist? (SE)
$\square$ Always
$\square \quad$ Often
$\square \quad$ Sometimes
$\square$ Rarely
$\square \quad$ Never

\section{Intraoperative Phase}

7. Which technique do you use for lumbar spinal fusion? (multiple options possible) (NL)

$\square$ unilateral/bilateral posterior lumbar interbody fusion (PLIF)

$\square$ unilateral/bilateral transforaminal lumbar interbody fusion (TLIF)

$\square \quad$ anterior lumbar interbody fusion (ALIF)

$\square$ extreme lateral lumbar interbody fusion (XLIF)

$\square \quad$ transaxial lumbar interbody fusion (AxiaLIF)

$\square \quad$ minimally invasive spinal fusion (MIP/MIS)

Which technique do you use for lumbar spinal fusion? (multiple options possible) (SE)

$\square \quad$ unilateral/bilateral posterior lumbar interbody fusion (PLIF)

$\square$ unilateral/bilateral transforaminal lumbar interbody fusion (TLIF)

$\square \quad$ anterior lumbar interbody fusion (ALIF)

$\square$ extreme lateral lumbar interbody fusion (XLIF)

$\square \quad$ transaxial lumbar interbody fusion (AxiaLIF)

$\square \quad$ minimally invasive spinal fusion (MIP/MIS)

$\square \quad$ other technique, which one:

8. Does the technique used for lumbar spinal fusion have an influence on your postoperative treatment? (NL)

$\square \quad$ No

$\square \quad$ Yes, in which way: 
Does the technique used for lumbar spinal fusion surgery have an influence on your postoperative treatment? (SE)

$\square$ no

$\square \quad$ yes $\Rightarrow$ If you have replied yes in question 8, in what way does the operation technique influence the post-operative mobilisation? (open question)

\section{Postoperative Phase - in Hospital}

9. When can the patient start to mobilise with respect to the used technique? (PO = postoperative) (NL)

\begin{tabular}{|l|c|c|c|c|c|c|}
\hline & Directly & 1st PO day & $2^{\text {nd } P O ~ d a y ~}$ & 3rd PO day & 24th PO day & $\begin{array}{c}\text { I do not use } \\
\text { this technique }\end{array}$ \\
\hline PLIF & $\square$ & $\square$ & $\square$ & $\square$ & $\square$ & $\square$ \\
\hline TLIF & $\square$ & $\square$ & $\square$ & $\square$ & $\square$ & $\square$ \\
\hline ALIF & $\square$ & $\square$ & $\square$ & $\square$ & $\square$ & $\square$ \\
\hline XLIF & $\square$ & $\square$ & $\square$ & $\square$ & $\square$ & $\square$ \\
\hline AxialLIF & $\square$ & $\square$ & $\square$ & $\square$ & $\square$ & $\square$ \\
\hline MIP/MIS & $\square$ & $\square$ & $\square$ & $\square$ & $\square$ & $\square$ \\
\hline
\end{tabular}

When can the patient start to mobilise with respect to the used technique? (PO = postoperative) (SE)

\begin{tabular}{|l|c|c|c|c|c|c|}
\hline & Directly & 1st PO day & 2 ${ }^{\text {nd }}$ PO day & 3rd PO day & Later & $\begin{array}{c}\text { Ido not use } \\
\text { this technique }\end{array}$ \\
\hline PLIF & $\square$ & $\square$ & $\square$ & $\square$ & $\square$ & $\square$ \\
\hline TLIF & $\square$ & $\square$ & $\square$ & $\square$ & $\square$ & $\square$ \\
\hline ALIF & $\square$ & $\square$ & $\square$ & $\square$ & $\square$ & $\square$ \\
\hline XLIF & $\square$ & $\square$ & $\square$ & $\square$ & $\square$ & $\square$ \\
\hline AxialLIF & $\square$ & $\square$ & $\square$ & $\square$ & $\square$ & $\square$ \\
\hline MIP/MIS & $\square$ & $\square$ & $\square$ & $\square$ & $\square$ & $\square$ \\
\hline
\end{tabular}

10. Is the patient mobilised by a physiotherapist in the hospital after the surgical procedure? (NL)
$\square \quad$ No
$\square \quad$ Yes

Is the patient mobilized by a physiotherapist in the hospital after the surgical procedure? (SE)
$\square \quad$ No
$\square \quad$ Yes 
11. On average - at what day is the following activity started? (NL)

\begin{tabular}{|l|c|c|c|c|c|c|}
\hline & 1st day & 2nd day & 3th day & 4th day & $\begin{array}{c}\text { Not } \\
\text { applicable }\end{array}$ & $\begin{array}{c}\text { With or without } \\
\text { supervision }\end{array}$ \\
\hline Sitting in bed & $\square$ & $\square$ & $\square$ & $\square$ & $\square$ & $\square$ with $\square$ without \\
\hline Standing beside the bed & $\square$ & $\square$ & $\square$ & $\square$ & $\square$ & $\square$ with $\square$ without \\
\hline Walking with support & $\square$ & $\square$ & $\square$ & $\square$ & $\square$ & $\square$ with $\square$ without \\
\hline $\begin{array}{l}\text { Walking without } \\
\text { support }\end{array}$ & $\square$ & $\square$ & $\square$ & $\square$ & $\square$ & $\square$ with $\square$ without \\
\hline Climbing Stairs & $\square$ & $\square$ & $\square$ & $\square$ & $\square$ & $\square$ with $\square$ without \\
\hline
\end{tabular}

On average - at what day are the following activities done? (SE)

\begin{tabular}{|l|c|c|c|c|}
\hline & 1st & 2nd & 3rd & 4th \\
\hline Sit in bed & PO & PO & $\square$ \\
\hline Stand at the bed & $\square$ & $\square$ & $\square$ & $\square$ \\
\hline Walk with support & $\square$ & $\square$ & $\square$ & $\square$ \\
\hline Walk without support & $\square$ & $\square$ & $\square$ & $\square$ \\
\hline Walk the stairs & $\square$ & $\square$ & $\square$ & $\square$ \\
\hline
\end{tabular}

From your answers in Question 12, do you think that the following activities should be supervised?

\begin{tabular}{|l|c|c|}
\hline & With supervision & Without supervision \\
\hline Sit in bed & $\square$ & $\square$ \\
\hline Stand next to the bed & $\square$ & $\square$ \\
\hline Walk with support & $\square$ & $\square$ \\
\hline Walk without support & $\square$ & $\square$ \\
\hline Walk in the stairs & $\square$ & $\square$ \\
\hline
\end{tabular}


12a. Is it allowed that a patient-after performing an activity - experiences pain? (NL)

$\square \quad$ No

$\square \quad$ Yes

Is it allowed that a patient-after performing an activity - experiences pain? (SE)

$\square \quad$ No

$\square \quad$ Yes

12b. How long is this increase allowed before you adjust the mobilisation strategy? (This question will only appear when "Yes" is selected at question 12a) (NL)
$\square<6$ hours
$\square \quad 6-24$ hours
$\square \quad 25-48$ hours
$\square \quad 49-72$ hours
$\square>72$ hours

If you consider pain to be allowed in relation to an activity (question 14) for how long do you allow that increase in pain to stay before adjusting the mobilization strategy? The pain can last for... (SE)
$\square<6$ hours
$\square \quad 6-24$ hours
$\square \quad 25-48$ hours
$\square \quad 49-72$ hours
$\square \quad>72$ hours

13. Based on what criteria do you determine when the patient can be discharged. If he/she is not suffering from wound leakage-and (NL):

$\square \quad$ The patient is able to stand independently and safely

$\square$ The patient is able to walk in his/her room independently and safely

$\square$ The patient is able to walk outside independently and safely

$\square$ The patient is able to walk stairs-with the use of one or two handrails-independently and safely

Based on what criteria do you determine when the patient can be discharged.

$\square \quad$ The patient can be discharged if he/she is not suffering from wound leakage-and (SE):

$\square$ The patient is able to stand independently and safely

$\square$ The patient is able to walk in his/her room independently and safely

$\square$ The patient is able to walk outside independently and safely

$\square$ The patient is able to walk stairs - independently and safely, with the use of one or two handrails

$\square \quad$ Other criteria, which: 


\section{Postoperative Phase - at home}

15. In the table below we ask you to indicate when a patient is allowed to perform the activity mentioned. This may be: 1-7 days, 1-4 weeks, 4-12 weeks, 3-6 months, > 6 months postoperatively (NL).

\begin{tabular}{|l|c|c|c|c|c|c|c|}
\hline & $1-7$ days & $\begin{array}{c}1-4 \\
\text { weeks }\end{array}$ & $\begin{array}{c}5-8 \\
\text { weeks }\end{array}$ & $\begin{array}{c}9-12 \\
\text { weeks }\end{array}$ & $\begin{array}{c}>3-5 \\
\text { months }\end{array}$ & $\begin{array}{c}>6 \\
\text { months }\end{array}$ & Never \\
\hline Walking & $\square$ & $\square$ & $\square$ & $\square$ & $\square$ & $\square$ & $\square$ \\
\hline Climbing stairs & $\square$ & $\square$ & $\square$ & $\square$ & $\square$ & $\square$ & $\square$ \\
\hline Running & $\square$ & $\square$ & $\square$ & $\square$ & $\square$ & $\square$ & $\square$ \\
\hline Bending forward & $\square$ & $\square$ & $\square$ & $\square$ & $\square$ & $\square$ & $\square$ \\
\hline Rotation to end position & $\square$ & $\square$ & $\square$ & $\square$ & $\square$ & $\square$ & $\square$ \\
\hline Extension to end position & $\square$ & $\square$ & $\square$ & $\square$ & $\square$ & $\square$ & $\square$ \\
\hline Cycling & $\square$ & $\square$ & $\square$ & $\square$ & $\square$ & $\square$ & $\square$ \\
\hline Driving car & $\square$ & $\square$ & $\square$ & $\square$ & $\square$ & $\square$ & $\square$ \\
\hline Swimming (breaststroke) & $\square$ & $\square$ & $\square$ & $\square$ & $\square$ & $\square$ & $\square$ \\
\hline Lifting (> 2.5 kg) & $\square$ & $\square$ & $\square$ & $\square$ & $\square$ & $\square$ & $\square$ \\
\hline Jumping (10 cm) & $\square$ & $\square$ & $\square$ & $\square$ & $\square$ & $\square$ & $\square$ \\
\hline
\end{tabular}

When do you allow a patient that has undergone lumbar fusion surgery to do the following activities? (SE)

14b. Maximum allowed lifting weight in $\mathrm{kg}$ : ...

If you think that a patient is allowed to lift, which is the maximal weight in $\mathrm{kg}$ ?

14c. Maximum allowed jumping weight in $\mathrm{cm}$ :

If you think that a patient is allowed to jump, which is the maximal height in $\mathrm{cm}$ ?

15a. Do your patients wear a lumbar corset after lumbar spinal fusion? (NL)
$\square \quad$ No
$\square \quad$ Yes

15b. In what situations do your patients wear a lumbar corset after lumbar spinal fusion? (multiple options possible) (NL)

$\square$ until 6 weeks postoperative

$\square$ until 3 months postoperative

$\square \quad$ until 1 year postoperative

$\square \quad$ Lifelong

$\square$ until there is a good gait

$\square$ until the patient is free of pain 
$\square$ choice of the patient

$\square$ during lifting

$\square \quad$ during physical activities

Do your patients use a brace after the lumbar fusion surgery? (SE)
$\square \quad$ No
$\square \quad$ Yes, to 6 weeks postoperatively
$\square \quad$ Yes, to 3 months postoperatively
$\square \quad$ Yes, to 1 year postoperatively
$\square$ Yes, until the patient can walk in a good way
$\square \quad$ Yes, until the patient is pain free
$\square \quad$ Yes, by lifting
$\square \quad$ Yes, for life
$\square \quad$ Yes, when physically active
$\square \quad$ The patient decides
$\square \quad$ Yes, when (write when):

15c. In what operation technique do your patients wear a lumbar corset (multiple options possible) (NL)
$\square$ unilateral/bilateral posterior lumbar interbody fusion (PLIF)
$\square$ unilateral/bilateral transforaminal lumbar interbody fusion (TLIF)
$\square \quad$ anterior lumbar interbody fusion (ALIF)
$\square \quad$ extreme lateral lumbar interbody fusion (XLIF)
$\square \quad$ transaxial lumbar interbody fusion (AxiaLIF)
$\square \quad$ minimally invasive spinal fusion

If you have answered yes in question 20 and your patients use a brace after lumbar fusion surgery, after what operation techniques do they use a brace? (multiple options possible) (SE)

$\square$ unilateral/bilateral posterior lumbar interbody fusion (PLIF)

$\square$ unilateral/bilateral transforaminal lumbar interbody fusion (TLIF)

$\square \quad$ anterior lumbar interbody fusion (ALIF)

$\square \quad$ extreme lateral lumbar interbody fusion (XLIF)

$\square \quad$ transaxial lumbar interbody fusion (AxiaLIF)

$\square \quad$ minimally invasive spinal fusion

16a. Do you prescribe physiotherapy in the postoperative phase? (NL)

$\square \quad \mathrm{No}$

$\square \quad$ Yes 
16b. After how many weeks do you start physiotherapy in the postoperative phase? (NL)
$\square$ Immediately
$\square \quad$ After 2 weeks
$\square \quad$ After 3-4 weeks
$\square \quad$ After 5-8 weeks
$\square \quad$ After 9-12 weeks
$\square \quad$ After $>12$ weeks

Do you prescribe physiotherapy in the postoperative phase? (SE)
$\square \quad$ No
$\square \quad$ Yes, immediately
$\square \quad$ Yes, after 2 weeks
$\square \quad$ Yes after 3-4 weeks
$\square \quad$ Yes, after $4-8$
$\square \quad$ yes, after 8-12 weeks
$\square \quad$ yes, after $>12$ weeks

17. What elements should NOT occur in the physical therapy? (multiple options possible) (NL)

$\square$ Posture correction

$\square$ Gait Practice

$\square$ Mechanical Diagnosis Therapy (McKenzie)

$\square$ Neuromotor control (Core Stability, Balance)

$\square$ Sensory stimulation (Massage)

$\square \quad$ Exercise therapy

$\square \quad$ Manuel therapy techniques

$\square \quad$ Physical form of therapy

$\square \quad$ Graded Activity

$\square$ Graded Exposure

$\square \quad$ Cognitive Behavourial Therapy

$\square \quad$ Alternative therapies, namely: 
What elements should NOT occur in the physical therapy? (multiple options possible) (SE)

$\square$ Posture correction

$\square$ Gait Practice

$\square \quad$ Mechanical Diagnosis Therapy (McKenzie)

$\square \quad$ Neuromotor control (Core Stability, Balance)

$\square$ Sensory stimulation (Massage)

$\square \quad$ Exercise therapy

$\square$ Manuel therapy techniques

$\square$ Physical form of therapy

$\square$ Graded Activity

$\square$ Graded Exposure

$\square$ Cognitive Behavourial Therapy

$\square \quad$ Alternative therapies, namely

$\square \quad$ No opinion

18. On what opinion do you base your advice to go back to work? (NL)

$\square \quad$ No opinion

$\square$ Time contingent-Gradual increase in hours regardless of pain

$\square \quad$ Pain contingent-Gradual increase in hours depending on level of pain

$\square \quad$ Full return to work, six weeks after the surgery

$\square \quad$ Related to the type of work

On what opinion do you base your advice to go back to work? (multiple answers possible) (SE)

$\square \quad$ No opinion

$\square \quad$ Time contingent - Gradual increase in hours regardless of pain

$\square$ Pain contingent - Gradual increase in hours depending on level of pain

$\square$ Full return to work, six weeks after the surgery

$\square \quad$ Related to the type of work

$\square \quad$ Other factors, name them here: 



\section{Chapter 8}

General discussion 



\section{GENERAL DISCUSSION}

The main goal of this thesis titled 'Back on Track: chronic low back pain rehabilitation in primary care' was to investigate the evidence for, and the effectiveness and feasibility of a biopsychosocial intervention in primary care provided by physiotherapists for patients with non-specific chronic low back pain (CLBP). First, a systematic review was performed to gather the evidence currently available. Second, a new biopsychosocial primary care intervention (Back on Track) was developed and an RCT was performed to compare its effectiveness in patients with less complex psychosocial complaints with usual primary care physiotherapy. In addition, the feasibility of the Back on Track intervention was investigated when provided as an integrated care approach to patients with moderate complex psychosocial complaints, usually referred to multidisciplinary secondary care treatments (i.e. substitution of care). Apart from conservative treatments, lumbar spinal fusion surgery is an increasingly provided treatment in patients with CLBP. It was investigated what opinions spinal surgeons have about (biopsychosocial) pre- and postoperative rehabilitation in patients with CLBP who undergo lumbar spinal fusion surgery.

In this chapter, the main findings of the studies will be presented, interpreted and discussed. Furthermore, an overview of methodological considerations, the implications for practice and future research will be provided. This chapter will end with the main conclusions.

\section{MAIN FINDINGS}

What is the evidence for the effect of a biopsychosocial intervention in primary care provided by physiotherapists?

Previous systematic reviews have confirmed modest but beneficial effects in improving pain and disability of a multidisciplinary biopsychosocial intervention in patients with CLBP., ${ }^{1,2}$ In primary care settings where patients might experience less complex psychosocial complaints, ${ }^{3}$ interventions provided by physiotherapists are usually less focused on psychosocial factors ${ }^{4}$ and physiotherapists are less specialised and confident in delivering a cognitive-behavioural approach, ${ }^{5}$ the evidence for the effectiveness of a biopsychosocial intervention is less conclusive. As described in Chapter 2 of this thesis, results of the systematic review showed moderate quality evidence for more effectiveness of a biopsychosocial primary care intervention as compared to education and advice in reducing functional disability, pain and work status at short, medium, and long term (3 studies, 991 participants). These findings indicate that patients in primary care might benefit more from a biopsychosocial physiotherapy programme than education and advice only. This finding is in line with a recent systematic review on the evidence for pain neuroscience education (PNE) in patients with chronic pain conditions. ${ }^{6}$ Louw et al. addressed that pain neuroscience education (PNE) improves knowledge about pain physiology and lowers catastrophizing thoughts, fear-avoidance and disability. However, when a physical component is added to PNE, therapy effects are optimised. Findings suggest that patients need an additional treatment containing 
a physical component in order to experience that the information provided in the education is applicable to their own situation. Patients will experience that being active and exercising is not harmful, and that they are able to be active and improve daily life functioning.

When a biopsychosocial primary care intervention is compared to a general physical activity intervention in patients with CLBP, only low-quality evidence is found for no difference in effectiveness in reducing pain and disability at short, medium and long term (4 studies, 435 participants). In addition, our randomised controlled trial (RCT, Chapter 5) showed no differences in effectiveness between a biopsychosocial primary care intervention and primary care physiotherapy as usual in improving functional disability, pain and psychological factors at short and medium term. Based on these findings, one might suggest that no physiotherapy intervention is more effective than another. So, when a patient is referred to a primary care physiotherapist, it seems of less importance what type of physiotherapy programme the patient should receive. It needs however be stressed that the evidence of our systematic review was of low-quality. Also our RCT did not achieve the required sample size and was therefore unable to accurately define the effects of the two interventions. The assumption that there are no differences in effects between a biopsychosocial intervention and other (usual) physical activity programmes should therefore be interpreted with caution and no final conclusions can be drawn.

Of notice is that patients included in the RCT, experienced psychosocial factors at baseline that only minimally influenced daily life functioning. When the Back on Track intervention was provided to patients in which psychosocial factors were slightly more complex, as in the feasibility study, the intervention did show significant beneficial effects in improving functional disability directly post-treatment as well as at 3 months follow-up (Chapter 6). Overall, the findings of the feasibility study in combination with previous mentioned studies may indicate that there is a continuum in effectiveness of a biopsychosocial intervention. It seems that with increasing complexity of psychosocial factors, the effectiveness of a biopsychosocial intervention increases accordingly. This also corresponds with previous research in which was shown that patients with higher fear-avoidance beliefs seem to benefit more from a fear-avoidance-based intervention than a usual programme (i.e. significantly reduce functional disability), while patients with less or no fear-avoidance beliefs benefit more from a usual programme?

\section{How feasible is a biopsychosocial intervention in a primary care physiotherapy setting?}

A biopsychosocial intervention in primary care may require a less sophisticated approach than a (multidisciplinary) biopsychosocial intervention in secondary care due the lower complexity of patient's psychosocial complaints. Nevertheless, it requires a certain attitude, behaviour and competence of physiotherapists. Literature suggest that primary care physiotherapists should be able to assess and identify psychosocial factors, understand the patient's situation, help defining achievable goals and reinforce positive coping behaviour. ${ }^{8}$ Furthermore, a biopsychosocial attitude is preferred over a biomedical attitude as this influences behaviour of physiotherapists. ${ }^{9}$ It has however been reported that primary care physiotherapists seem to have difficulties with discussing psychosocial factors. ${ }^{5}$ Moreover, many physiotherapists prefer a rather "straight forward" biomedical approach than a biopsychosocial approach. ${ }^{5}$ To direct primary care physiotherapists 
into a biopsychosocial approach and to improve competence and confidence, literature recommends training and additional support., From our systematic review, it became clear that previous studies offered training programmes to primary care physiotherapists of 2 to 4 days. ${ }^{10-14}$ Also physiotherapists in our Back on Track studies received 3 education sessions ( 12 hours in total). The fact however that training is received does not guarantee that the intervention will be provided as intended. Many studies however, did not report (and possibly did not investigate) to what extent the intervention was sufficiently provided. This practical information would have helped to interpret study outcomes and to identify the feasibility of a biopsychosocial intervention in primary care. ${ }^{15}$ From the studies that did report on treatment fidelity it can be concluded that physiotherapists delivered a biopsychosocial intervention sufficiently as soon as physiotherapists received, in addition to the training programme and treatment manual, support prior and during the intervention. ${ }^{11}$ Physiotherapists in the study of Lamb et al. ${ }^{11}$ for example received supervision on site, a DVD with examples of the sessions, a website with information, and had contacts via phone or email with a clinical researcher. Physiotherapists in the Back on Track studies also received next to the initial training and detailed treatment manual, two booster sessions and support by the educational team if needed. From the Back on Track feasibility study (Chapter 6), it became clear that physiotherapists sufficiently delivered the essential (and unique) elements of the Back on Track protocol. It can therefore be concluded that a biopsychosocial primary care intervention is feasible to provide by primary care physiotherapists if physiotherapists are trained in delivering a biopsychosocial intervention, receive a detailed treatment protocol and receive support prior and during the intervention in addition.

During the feasibility study, the Back on Track intervention was offered as an integrated care approach. This means, collaboration and communication between physicians in rehabilitation medicine and physiotherapists was facilitated (i.e. with an online data-management and communication tool) and a final consultation with the physician in rehabilitation medicine was added at the end of the Back on Track intervention. The integrated care approach was deemed necessary due to the slightly higher complexity of the psychosocial complaints of patients eligible for the feasibility study. During the study, it became evident that the online communication tool was not optimal. Therefore, physiotherapists contacted physicians mainly by email, although this occurred only occasionally. In addition to the remark regarding the communication tool, the final consultation with the physician was frequently not attended by patients. The reason for the number of no-shows was mostly unclear. Based on abovementioned findings, it may be suggested that although a biopsychosocial primary care intervention seemed feasible to provide by primary care physiotherapists, the integrated care approach seemed less implemented as planned. It requires attention and should be improved to ensure actual integration of primary and secondary care in future.

\section{Biopsychosocial approach in combination with a surgical intervention}

As mentioned previously, although conservative treatments are generally recommended over lumbar spinal fusion in the initial stage of patients with CLBP, the number of lumbar spinal fusion surgeries has increased remarkably in the past decades. ${ }^{16-18}$ The indication for spinal fusion 
surgery is ill-defined and less consensus exists regarding clinical decision making. ${ }^{19}$ Our cross-sectional survey among spinal surgeons in the Netherlands and Sweden (Chapter 7) added further evidence that large variability exists between surgeons regarding pre- and postoperative rehabilitation. Spinal surgeons seem to have varying ideas about preoperative physiotherapy (whether it should be recommended or not), and what activities, sports and physiotherapy interventions are allowed postoperatively (and at what time point and to what intensity). Prehabilitation for example, was in both countries only structurally recommended by one third of the surgeons. Preoperative rehabilitation ("prehabilitation") has been shown to stimulate faster recovery (e.g. walking, stair climbing) ${ }^{20}$ and hospital discharge. ${ }^{20,21}$ When cognitive-behavioural elements are added, patients furthermore seem to recover faster with less use of analgesic. ${ }^{22}$ The pain intensity remains similar to patients without prehabilitation, but this might indicate that a prehabilitation programme with cognitive-behavioural elements stimulates patients to better cope with their pain. Prehabilitation is however a rather novel approach and less investigated yet. ${ }^{23}$ This might be an explanation why prehabilitation is currently less integrated in surgeons' daily practice in the Netherlands and Sweden.

Regarding postoperative rehabilitation, physiotherapy results in faster recovery at short and long term as compared to usual care (i.e. advice and brief physical programme to prevent deep vein thrombosis. ${ }^{24}$ Despite the beneficial effects, only $44 \%$ of the Dutch referred patients to a post-operative physiotherapy programme. In Sweden, referral to postoperative physiotherapy was more consistently recommended as $88 \%$ of the surgeons indicated that they did. From those who did refer to a postoperative physiotherapy programme, one Dutch spinal surgeon and one third of the Swedish spinal surgeons had no opinion on what type of therapy this should be. Literature has shown that for the postoperative phase a biopsychosocial approach can optimise recovery as well. It seems to improve functional disability and fear of movement/injury to a significantly greater extent than a pain-contingent exercise approach. ${ }^{25}$ Our survey did not provide data on what type of physiotherapy programmes were usually recommended in the Netherlands and Sweden. Based on the available data it can however be concluded, that nearly all surgeons in both countries at least did not discourage cognitive-behavioural therapy.

\section{METHODOLOGICAL CONSIDERATIONS}

Overall, RCTs are generally the gold standard to detect causal relationships. Due to its randomisation procedure, confounders (known and unknown) will be equally distributed. ${ }^{26}$ Furthermore, if allocation is concealed and participants, professionals and data analysts involved remain blinded, there is less potential for bias. A drawback of an RCT is the rather large sample size required. In our RCT, sample size calculation resulted in 86 patients (total number). During the study however, only 25 patients were included for participation. Patients eligible for the RCT needed to experience psychosocial factors that minimally influenced daily life functioning. We aimed to recruit this specific subgroup of patients as these patients were usually referred to primary care physiotherapy practices which would reflect daily practice best. Furthermore, identifying the effectiveness of an intervention in a homogeneous subgroup of patients is increasingly recommended by researchers and clinicians. ${ }^{8}$ Dutch legislation for this subgroup of patients 
however changed during the time we submitted our study protocol and received medical ethical approval. Referral to secondary care was discouraged which resulted in the referral of less patients to physicians in rehabilitation medicine (i.e. our recruitment pathway). The inability to achieve the calculated sample size had eventually detrimental consequences for the RCT. The low sample size increased the risk for a type II error (false negative conclusions); the possibility that there were differences in effects, but the study was unable to detect them. ${ }^{27}$

In addition to the low sample size, the low recruitment rate caused patients to wait for group sessions in the Back on Track intervention, resulting in less motivated patients and an increased number of patients discontinuing the intervention. The standardised design of our RCT limited usual practice of physicians, i.e. providing therapy information. Physicians were blinded for the randomisation sequence (i.e. concealed allocation), therefore did not know to which therapy patients would be allocated to. As a consequence, physicians could not provide in-depth therapy information to the patient as they would usually do. It became evident that therapy expectations of patients regarding the biopsychosocial intervention did not match the actual content of the therapy (i.e. what they experienced). Some patients thought that the focus was less on psychological factors and more time would be directed to physical activities. Such expectations probably match better with a regular physiotherapy programme instead of a biopsychosocial approach. The mismatch in what patients expected from and experienced during the Back on Track intervention resulted in discontinuance of the intervention in several patients in the RCT. The number of patients in the physiotherapy as usual group who discontinued the intervention was lower. Based on these mentioned experiences, it is likely that the standardised RCT procedure in combination with the low recruitment rate limited clinical practice, especially in the Back on Track intervention. The study may therefore have reflected the actual situation less.

In contrast to the RCT design, the feasibility study used a one group pre-post test design. As compared to a RCT design, a group pre-post test design has several methodological disadvantages. The lack of a control group and randomisation procedure leads to non-blinding (an increased risk for bias) and hampers detecting a causal relationship between the intervention and therapy effects. It remains unknown whether the change in the level of functional disability, detected in the feasibility study, was a direct result of the Back on Track intervention or a result of the natural course of CLBP. As a result, the feasibility study cannot draw conclusions regarding causal effect. A one group pre-post test design can however still be very useful for exploratory reasons. Such a study is easier to monitor/conduct due to the non-randomisation and non-blinding procedures. A new intervention can be merged into a usual clinical setting and therefore reflects the actual setting best (i.e. improving external validity). Patients in the feasibility study, for example, could be better prepared than patients in the RCT as they were not randomised to two interventions and eventually showed less discontinuance due to wrong expectations. A one group pre-post test design can therefore give, with lower burden and in relatively short-time, direction to whether a newly developed intervention has potency in being effective in a usual care setting, and what barriers and facilitators are encountered while implementing. Information can subsequently be used to optimise implementation if desirable, or to develop future studies aiming to detect causal relationships. 
An essential inclusion criterion to be eligible for the RCT and feasibility study was the criterion to experience low or moderate complex psychosocial complaints influencing daily life functioning at least moderately. This inclusion criterion was based on the WPN-classification system to reflect usual practice. The WPN-classification is currently in use in daily care by physicians in rehabilitation medicine (described in the introduction of this thesis). Assigning a specific WPN-classification to the patient is based on subjective decision-making and research reported a low inter-rater reliability. ${ }^{28}$ No clinical decision tool is available for physicians in rehabilitation medicine to support their classification. It was therefore chosen to provide mean range scores for several questionnaires such as pain intensity (Numeric Rating Scale; NRS), catastrophizing (Pain Catastrophizing Scale; PCS), anxiety and depression (Hospital Anxiety and Depression Scale; HADS) and physical functioning (RAND-36). These scores would reflect an average person eligible for the RCT and feasibility study. Physicians however informed that range scores or questionnaires were frequently not used in their decision regarding the classification as questionnaires did not always accurately reflect the current status of patients. The classification of physicians was therefore largely based on clinical reasoning. Apart from which approach was used to classify patients, the comparison of baseline values of patients included in both studies showed that patients referred to the RCT reported (on average) less complex psychosocial complaints than the patients referred to the feasibility study.

Some patients in the RCT, however, experienced such low levels of anxiety and depression that it might have introduced floor effects (HADS, 0-21 per subscale). A floor effect is present when $>15 \%$ of the patients report the lowest score on a measurement instrument. ${ }^{29}$ Patients in the RCT reported a median baseline anxiety score of 4 (range 0-18) and a mean depression score of 3.9 (SD 3.0). Twenty-four percent of the patients reported a minimum level of anxiety (scoring 0-2), and $40 \%$ a similar level of depression. The floor effect in the HADS may be one reason why no significant differences in improvements in the HADS could be detected between the interventions.

The primary outcome in the RCT and feasibility study was functional disability, measured with the Quebec Back Pain Disability Scale (QBPDS). The primary aim was to determine whether the change in functional disability would be statistically significant between interventions (RCT) and over time (feasibility). A statistically significant improvement at group level is however not necessarily clinically relevant for a patient. Determining the clinically relevance of improvements can be of significant value in addition to statistical testing. Multiple studies have reported a minimal clinical important change (MCIC) for the QBPDS ranging from 3 to 32.9. ${ }^{30-33}$ Due to the fact the baseline level can remarkably influence the level of improvement within a subject, studies recommended to take into account the baseline scores of patients when defining the $\mathrm{MCIC}$. An expert panel proposed a MCIC of $30 \%$ from baseline, ${ }^{34}$ while Demoulin et al. ${ }^{30}$ proposed an optimal cut-off point for three baseline clusters specifically (i.e. 3, 5 and 10 points for patients with a QBPDS baseline of $<40, \geq 40-\leq 50$, and $>50$, respectively). When both approaches were applied to data from the RCT it became evident that $3 / 8$ responders (38\%) of the Back on Track intervention and 3/10 responders (30\%) in the physiotherapy as usual group achieved a MCIC of $30 \%$ at post-treatment. When considering a MCIC based on the cluster cut-off points of Demoulin et al., remarkably more responders in the Back on Track intervention (6/8 responders, $75 \%)$ as 
compared to the physiotherapy group (3/10, 30\%) achieved a MCIC. This finding indicates that the latter approach leads to an overall higher number of patients achieving a MCIC (i.e. increased sensitivity), and furthermore suggest that the Back on Track intervention would be more beneficial than physiotherapy as usual at post-treatment. Also in the feasibility study, 5/9 responders (56\%) achieved a MCIC of 30\% at post-treatment, while 6/9 responders (67\%, 1 extra) achieved a MCIC based on the cluster cut-off points. The difference in approaches used was even larger at 3 months as 3/10 responders (30\%) achieved a MCIC of 30\%, and 6/10 responders (60\%, 3 extra) based on the cluster cut-off points. The specific MCIC approach used can therefore have remarkable consequences how data are interpreted and presented. Indeed, sensitivity values of the cluster-based approach (ranging from 0.778 to 0.786 ) are higher than those of the $30 \%$ approach (0.543). This, in combination with the lower specificity (0.758 to 0.827 ) as compared to the $30 \%$ approach (0.937) likely explains the differences in the number of patients achieving a MCIC. In patients with CLBP who are functionally disabled for longer duration such as in the Back on Track studies, a smaller improvement might be relevant (high sensitivity). ${ }^{30}$ Based on this reasoning, a cluster-based approach might be most applicable in our group of patients with CLBP. Overall, it should however still be stressed that, irrespective of what MCIC approach would be used, the RCT and feasibility studies faced a rather large number of missing data. The results regarding the $\mathrm{MCIC}$ presented above (ratios and percentage scores) should therefore be interpreted very carefully.

As described in the RCT protocol (Chapter 4) we initially aimed to evaluate the differences in cost-effectiveness at long term follow-up (12 months follow-up) between the Back on Track intervention and primary care physiotherapy as usual. Long term follow-up data was however not yet available to perform this analysis. Moreover, to what extent a cost-effectiveness study would add value in future is questionable as the sample size is small and response rates are low.

The cross-sectional study in Dutch and Swedish spinal surgeons gave valuable insight in the current spinal surgeon's opinions regarding pre- and postoperative rehabilitation in the Netherlands and Sweden. The survey was first developed in English and send to Dutch spinal surgeons. Afterwards the survey was translated into Swedish (forward translation) and send to Swedish spinal surgeons. One remark to this approach was the lack of backward translation. Backward translation decreases the risk for questionnaires not being an adequate reflection of the original version..$^{29}$ It remains unclear to what extent this influenced study outcomes, however as the Dutch questionnaire was formulated in English (and not Dutch) this possibly facilitated forward translation. Eventually few differences were visible between the questionnaires, mainly present in answering scales. These differences were a result of the recommendations of the Swedish advisory board. This eventually did not influence study results as the aim was to narratively report findings.

\section{IMPLICATIONS FOR PRACTICE}

For patients in whom psychosocial factors are of low complexity and minimally influence daily life functioning, conclusive evidence is available that a biopsychosocial intervention is more effective than education and advice. On the other hand, inconclusive evidence is available about what type of physiotherapy programme should be provided in clinical practice. As mentioned 
previously, new studies should be performed to provide well-grounded recommendations for this group of patients specifically. For patients in which psychosocial factors are of moderate complexity, and who are usually referred to secondary rehabilitation treatments, a biopsychosocial intervention is potentially effective. Substituting care for this group of patients might therefore have potency. However, first the (cost)-effectiveness should be investigated as compared to a usual multidisciplinary intervention in secondary care. As soon as the integrated Back on Track intervention shows equal effects but less costs, this integrated biopsychosocial care approach should be recommended (and should be implemented) over a usual secondary program. If such a program will be implemented in daily physiotherapy practice, training and supporting physiotherapists in order to change or increase their orientation to a more behavioural/biopsychosocial approach is desirable. A training of approximately 2 days and sufficient additional support (treatment manual, examples of cases, coaching on the job) seem to be sufficient. Generally stated, a biopsychosocial orientation of physiotherapist might be recommended for every physiotherapist in primary care as it may facilitate identification of psychosocial factors in those patients not referred by a physician in rehabilitation medicine, but a general practitioner (GP) or those without referral (i.e. direct access). Additional screening tools could be useful to support clinical decision making regarding the type and location (e.g. primary/secondary care) of intervention. ${ }^{35,36}$ Such screening tools can also be useful to objectify the decision making of the physicians in rehabilitation medicine (secondary care) although these need to be developed in future.

If a biopsychosocial intervention like the Back on Track intervention is indicated, the referring or treating health care professional should determine the patient's biopsychosocial profile but moreover his/her expectations regarding the biopsychosocial intervention. Patient's expectations might be more biomedically, physically oriented rather than biopsychosocially, educative oriented. Anticipating on patient's expectations and providing information about pain management might facilitate compliance during therapy. Pain education, which was initially protocolled for one individual session may require an additional session as it may facilitate flexibility, interaction and clarity in patients. Although education and advice about the role of catastrophic thoughts and avoidance behaviour on daily life function seem beneficial to provide and should be identified by physiotherapists as well, the extent to which actual exposure to fearful activities is needed and should be provided as a protocolled session during physical active sessions may depend on the presence of dysfunctional beliefs and behaviour in patients. Group therapy should moreover be provided only if sufficient eligible patients are referred to the intervention, and practice facilities (and planning) are sufficient. At the end of the intervention, it should be indicated whether a patient requires referral (back) to a physician in rehabilitation medicine. Communication and collaboration, especially in case of an integrated care programme, should be transparent and optimal. Health care professionals might benefit from the use of similar reports and sources of information. Furthermore, an interim contact between health care professionals, whether or not in combination with patients, could be valuable to integrate clinical expertise from different settings, to reinforce the current approach used and/or to define future management. In 
case a patient is directed towards lumbar spinal fusion surgery, surgeons should find consensus on prehabilitation (and what type), as well as postoperative mobilisation and rehabilitation (what time point, type, intensity, and etcetera). It is recommended to investigate the effectiveness of different approaches, to come to general agreement, and develop clinical guidelines. This likely stimulates uniform clinical practice and improves quality of care.

\section{IMPLICATIONS FOR FURTHER RESEARCH}

As mentioned previously, a new study with sufficient power remains necessary to finally answer the question which type of physiotherapy treatment in primary care is most effective and should be recommended to patients with low complex psychosocial complaints. Recruitment of this group of patients is likely to be enhanced when recruitment occurs via GPs and primary care physiotherapists, supported with clinical decision tools. With regard to patients with moderate complex psychosocial complaints, a future study is required to investigate the differences in cost-effectiveness between a biopsychosocial integrated care approach with usual multidisciplinary (secondary) care. Recruitment should occur by physicians in rehabilitation medicine considering the biopsychosocial profile. With regard to the pre- and postoperative rehabilitation in lumbar spinal fusion surgery, future studies are needed to investigate the differences in effectiveness between different types of approaches (biopsychosocial approach or usual/physical approach, pre- and postoperative), its timing, frequency and intensity.

What type of design should be used in future studies is debatable. Although an RCT may be considered as the leading design to detect differences between interventions, it requires a rather large sample size and adaptations from usual procedures. An alternative research design to counteract the difficulties with the required sample size is a single-case design. Such design requires a lower sample size as the included patients functions as their own control. ${ }^{37,38}$ On the other hand, it requires multiple measurements within short time intervals to detect changes in effects (e.g. daily or multiple measurements per week). The increased burden for participating patients and complexity of study logistics should not be underestimated. Moreover, as mentioned previously, an RCT design (as well as a single-case design), has one important disadvantage; it standardised procedure which limits reflection of usual practice. In the field of intervention-research, studies are especially valuable when performed in a situation that resembles daily practice. This facilitates generalisation of study results into practice. For clinical purposes, it may be recommended to use a longitudinal study design in which patients in one (geographical) area receive the intervention of interest, and in another area receive the control intervention. Less adaptations in usual procedures are needed and patients can start the intervention well-prepared. A mixed method design considering the (cost)-effectiveness (quantitative data) and experiences from patients and health care professionals (qualitative data) will furthermore give comprehensive insight what can be used to actually improve health care. Such approach is in line with Triple Aim concept which supports evaluation and improvements in (1) patient's health, (2) perceived quality of care, and (3) health care related costs. ${ }^{39}$ 


\section{FINAL CONCLUSIONS}

In summary, this thesis showed, for patients with CLBP experiencing low complex psychosocial complaints, more effectiveness of a biopsychosocial primary care intervention over education and advice, and inconclusive evidence regarding the effectiveness of a biopsychosocial approach as compared to usual physiotherapy in primary care. Furthermore, an integrated biopsychosocial care intervention does seem to have beneficial effects in improving functional disability in patients with moderate complex psychosocial complaints, although its effectiveness needs to be compared with a usual (multidisciplinary) programme in secondary care in future. Physiotherapists in primary care settings are able to sufficiently deliver a biopsychosocial primary care intervention, but need sufficient training and support. To ensure an integrated care approach, collaboration and communication between physiotherapists and physicians should furthermore be improved. According to Dutch and Swedish spinal fusion surgeons, biopsychosocial rehabilitation seem less integrated in current lumbar spinal fusion rehabilitation and variability in practice is especially reported in postoperative phase. 


\section{REFERENCES}

1. Kamper SJ, Apeldoorn AT, Chiarotto A, et al. Multidisciplinary biopsychosocial rehabilitation for chronic low back pain. The Cochrane database of systematic reviews. 2014;9:CD000963.

2. Hall A, Richmond H, Copsey B, et al. Physiotherapist-delivered cognitive-behavioural interventions are effective for low back pain, but can they be replicated in clinical practice? A systematic review. Disability and rehabilitation. 2016:1-9.

3. Boonstra AM, Bühring M, Brouwers M, Bosma F, Schiphorst Preuper R. Patiënten met chronische pijnklachten op het grensvlak van revalidatiegeneeskunde en psychiatrie. Ned Tijdschr Pijn bestr. 2008;27(34):5-9.

4. Swinkels IC, van den Ende CH, van den Bosch W, Dekker J, Wimmers RH. Physiotherapy management of low back pain: does practice match the Dutch guidelines? The Australian journal of physiotherapy. 2005;51(1):35-41.

5. Synnott A, O'Keeffe M, Bunzli S, Dankaerts W, O'Sullivan P, O'Sullivan K. Physiotherapists may stigmatise or feel unprepared to treat people with low back pain and psychosocial factors that influence recovery: a systematic review. J Physiother. 2015;61(2):68-76.

6. Louw A, Zimney K, Puentedura EJ, Diener I. The efficacy of pain neuroscience education on musculoskeletal pain: A systematic review of the literature. Physiother Theory Pract. 2016;32(5):332-355.

7. George SZ, Fritz JM, Bialosky JE, Donald DA. The effect of a fear-avoidance-based physical therapy intervention for patients with acute low back pain: results of a randomized clinical trial. Spine. 2003;28(23):2551-2560.

8. van der Windt D, Hay E, Jellema P, Main C. Psychosocial interventions for low back pain in primary care: lessons learned from recent trials. Spine. 2008;33(1):81-89.

9. Darlow B, Fullen BM, Dean S, Hurley DA, Baxter GD, Dowell A. The association between health care professional attitudes and beliefs and the attitudes and beliefs, clinical management, and outcomes of patients with low back pain: a systematic review. European journal of pain. 2012;16(1):3-17.

10. McDonough SM, Tully MA, Boyd A, et al. Pedometer-driven walking for chronic low back pain: a feasibility randomized controlled trial. The Clinical journal of pain. 2013;29(11):972-981.

11. Lamb SE, Hansen Z, Lall R, et al. Group cognitive behavioural treatment for low-back pain in primary care: a randomised controlled trial and cost-effectiveness analysis. Lancet. 2010;375(9718):916-923.

12. Johnson RE, Jones GT, Wiles NJ, et al. Active exercise, education, and cognitive behavioral therapy for persistent disabling low back pain: a randomized controlled trial. Spine. 2007;32(15):1578-1585.

13. Macedo LG, Latimer J, Maher CG, et al. Effect of motor control exercises versus graded activity in patients with chronic nonspecific low back pain: a randomized controlled trial. Physical therapy. 2012;92(3):363377.

14. van der Roer N, van Tulder M, Barendse J, Knol D, van Mechelen W, de Vet H. Intensive group training protocol versus guideline physiotherapy for patients with chronic low back pain: a randomised controlled trial. European spine journal : official publication of the European Spine Society, the European Spinal Deformity Society, and the European Section of the Cervical Spine Research Society. 2008;17(9):1193-1200.

15. Saunders RP, Evans MH, Joshi P. Developing a process-evaluation plan for assessing health promotion program implementation: a how-to guide. Health promotion practice. 2005;6(2):134-147. 
16. Stromqvist B, Fritzell P, Hagg O, Jonsson B, Sanden B, Swedish Society of Spinal S. Swespine: the Swedish spine register : the 2012 report. European spine journal : official publication of the European Spine Society, the European Spinal Deformity Society, and the European Section of the Cervical Spine Research Society. 2013;22(4):953-974.

17. Rajaee SS, Bae HW, Kanim LE, Delamarter RB. Spinal fusion in the United States: analysis of trends from 1998 to 2008. Spine. 2012;37(1):67-76.

18. Du Bois M, Szpalski M, Donceel P. A decade's experience in lumbar spine surgery in Belgium: sickness fund beneficiaries, 2000-2009. European spine journal : official publication of the European Spine Society, the European Spinal Deformity Society, and the European Section of the Cervical Spine Research Society. 2012;21(12):2693-2703.

19. Willems P, de Bie R, Oner C, Castelein R, de Kleuver M. Clinical decision making in spinal fusion for chronic low back pain. Results of a nationwide survey among spine surgeons. BMJ Open. 2011;1 (2):e000391.

20. Nielsen PR, Jorgensen LD, Dahl B, Pedersen T, Tonnesen H. Prehabilitation and early rehabilitation after spinal surgery: randomized clinical trial. Clinical rehabilitation. 2010;24(2):137-148.

21. Santa Mina D, Matthew AG, Hilton WJ, et al. Prehabilitation for men undergoing radical prostatectomy: a multi-centre, pilot randomized controlled trial. BMC Surg. 2014;14:89.

22. Rolving N, Nielsen CV, Christensen FB, Holm R, Bunger CE, Oestergaard LG. Preoperative cognitive-behavioural intervention improves in-hospital mobilisation and analgesic use for lumbar spinal fusion patients. BMC musculoskeletal disorders. 2016;17:217.

23. Lotzke $H$, Jakobsson M, Brisby $H$, et al. Use of the PREPARE (PREhabilitation, Physical Activity and exeRcisE) program to improve outcomes after lumbar fusion surgery for severe low back pain: a study protocol of a person-centred randomised controlled trial. BMC musculoskeletal disorders. 2016;17(1):349.

24. McGregor AH, Probyn K, Cro S, et al. Rehabilitation following surgery for lumbar spinal stenosis. A Cochrane review. Spine. 2014;39(13):1044-1054.

25. Abbott AD, Tyni-Lenne R, Hedlund R. Early rehabilitation targeting cognition, behavior, and motor function after lumbar fusion: a randomized controlled trial. Spine. 2010;35(8):848-857.

26. Higgins JPT, Green S. Cochrane Handbook for Systematic Reviews of Interventions. 2011.

27. Christensen E. Methodology of superiority vs. equivalence trials and non-inferiority trials. J Hepatol. 2007;46(5):947-954.

28. Boonstra AM, Schiphorst Preuper HR, Brouwer M, Weerdt de M, Knol DL, Köke A. Classificatie van patiënten met chronische pijnklachten; onderzoek naar de betrouwbaarheid van de WPN niveaus. 2011;30 (45):5-10.

29. De Vet HCW, Terwee CB, Mokkink LB, Knol DL. Measurement in Medicine: a practical guide. Cambridge: Cambridge University Press; 2011.

30. Demoulin C, Ostelo R, Knottnerus JA, Smeets RJ. Quebec Back Pain Disability Scale was responsive and showed reasonable interpretability after a multidisciplinary treatment. Journal of clinical epidemiology. 2010;63(11):1249-1255.

31. Kopec JA, Esdaile JM, Abrahamowicz M, et al. The Quebec Back Pain Disability Scale. Measurement properties. Spine. 1995;20(3):341-352.

32. van der Roer N, Ostelo RW, Bekkering GE, van Tulder MW, de Vet HC. Minimal clinically important change for pain intensity, functional status, and general health status in patients with nonspecific low back pain. Spine. 2006;31(5):578-582. 
33. Davidson M, Keating JL. A comparison of five low back disability questionnaires: reliability and responsiveness. Physical therapy. 2002;82(1):8-24.

34. Ostelo RW, Deyo RA, Stratford P, et al. Interpreting change scores for pain and functional status in low back pain: towards international consensus regarding minimal important change. Spine. 2008;33(1):90-94.

35. Linton SJ, Nicholas M, MacDonald S. Development of a short form of the Orebro Musculoskeletal Pain Screening Questionnaire. Spine. 2011;36(22):1891-1895.

36. Hill JC, Dunn KM, Lewis M, et al. A primary care back pain screening tool: identifying patient subgroups for initial treatment. Arthritis and rheumatism. 2008;59(5):632-641.

37. Onghena P, Edgington ES. Customization of pain treatments: single-case design and analysis. The Clinical journal of pain. 2005;21(1):56-68; discussion 69-72.

38. Kratochwill TR, Hitchcock J, Horner RH, et al. Single-case design technical documentation. 2010; What Works Clearinghouse website: http://ies.ed.gov/ncee/wwc/pdf/wwc_scd.pdf.

39. Berwick DM, Nolan TW, Whittington J. The triple aim: care, health, and cost. Health Aff (Millwood). 2008;27(3):759-769. 



\section{Chapter 9}

Valorisation addendum 



\section{VALORISATION ADDENDUM}

In this thesis, we focussed on therapy approaches delivered by primary care physiotherapists to improve the level of daily activities in patients with non-specific chronic low back pain (CLBP). We investigated whether a biopsychosocial intervention provided by a primary care physiotherapist would be effective in reducing the level of functional disability. Furthermore, we investigated whether a biopsychosocial intervention would be feasible to implement when offered as an integrated care intervention. An integrated care intervention means that the intervention is provided by a primary care physiotherapist in collaboration with a physician in rehabilitation medicine. While the previous chapters described the findings of the studies, the purpose of this chapter, the valorisation addendum, is to describe the relevance of the thesis findings and describe to whom the results are applicable, how research findings can be translated into innovative products and activities, and how implementation can be realised.

\section{RELEVANCE OF THE FINDINGS}

Although the studies described in this thesis were performed from a scientific point of view, the research findings about which physiotherapy approach is most beneficial for patients with CLBP is useful to optimize current health care. Patients benefit from optimized health care as they will improve their level of functional disability to a greater extent and will perform better at home and at work. This in turn can have a significant impact at the individual, societal and economic level. Based on the research findings described in this thesis, we concluded that, for patients with a psychosocial profile of low complexity, the current primary care physiotherapy seems to suffice and therefore does not need to be transformed into a more holistic approach which requires extra (biopsychosocial) training and supervision of physiotherapists. For patients with a psychosocial profile of moderate complexity, promising results were found for our biopsychosocial care intervention when offered as an integrated care approach. This subgroup of patients usually receives multidisciplinary care in a secondary care setting (e.g. hospital or rehabilitation clinic). The integrated care intervention significantly improved the level of functional disability in this subgroup of patients. Furthermore, after training, primary care physiotherapists were able to deliver a biopsychosocial intervention in a qualitative sufficient way. Based on these findings, a biopsychosocial integrated care intervention might be a good alternative for the (more expensive and intensive) multidisciplinary care usually provided. Although these two interventions need to be compared in a future longitudinal study preferably using a randomised controlled study design and cost-effectiveness analysis, the next sections will describe what kind of impact a biopsychosocial integrated care intervention could have and for whom it could be implemented in daily practice. 


\section{TARGET GROUP}

Implementing a biopsychosocial integrated care intervention in future can affect different kinds of populations. Examples are patients who receive the intervention, primary care physiotherapists who deliver the intervention, physicians in rehabilitation medicine, general practitioners (GP's) or other health care specialists who refer patients (and partly deliver the intervention), and health care insurance companies who fund the intervention.

Regarding patients, it should be mentioned that CLBP is a broad concept. Previous chapters already explained that within the population of patients with CLBP different psychosocial complaints and different levels of functional disability can be present. The integrated care intervention was specifically developed for patients with a psychosocial profile of moderate complexity. Implementing an integrated care intervention will therefore be applicable to this group of patients only. Some speculations can be made regarding the advantages of implementation for this group of patients. First, patients might be able to start rehabilitation at an earlier point in time. Hospitals or rehabilitation clinics often deal with a waiting list, while physiotherapy practices (in which the integrated care intervention will be offered) often do not. When patients can start therapy directly, patients will start rehabilitation at an earlier moment stimulating earlier return to work and social activities. A second advantage for patients is the shorter distance to a physiotherapy practice than a rehabilitation clinic or hospital. This leads to less traveling time and traveling costs.

Regarding physiotherapists working in primary care physiotherapy settings it should be mentioned that many physiotherapists have no or less experience with providing a protocolled biopsychosocial intervention and often have a more biomedical than biopsychosocially oriented attitude regarding back pain. Furthermore, regular applied physiotherapy sessions are generally provided individually (no group therapy) and with limited or no cooperation with other health care specialists. Implementing a biopsychosocial intervention with an integrated care approach can therefore have large impact on the current practice of primary care physiotherapists. Effort from physiotherapists is required to follow an educational programme, to become more biopsychosocially oriented and to achieve sufficient competence in providing biopsychosocial treatment elements. Also throughout the intervention, physiotherapists need to pay attention to adhere to the protocol, to plan and organise the protocolled (group) sessions, and to keep the level of communication sufficient with the physician in rehabilitation medicine. Although implementing a biopsychosocial intervention requires effort from physiotherapists, they might benefit from it as well. Working according the latest scientific evidence and being in close contact with the health care specialist likely increases the quality of care and satisfaction of patients as well as their own work satisfaction. On the long term, positive experiences of patients and professional colleagues might result in an increased number of (referred) patients to their practice.

In addition to the physiotherapists, implementation of the integrated care intervention affects the referring health care specialists, i.e. the physician in rehabilitation medicine. The physician in rehabilitation medicine provides medical education (prepares a patient for a biopsychosocial approach) and refers the patient to the therapy. During the biopsychosocial integrated care intervention, the physician supports the rehabilitation process throughout. Since therapy 
is provided at different therapy settings and not within the same institute as usually would be the case, it requires some extra time and effort to keep in close contact with the patient and the treating physiotherapist.

Another target population that will be involved when implementing the biopsychosocial integrated care intervention are the group of health insurance companies. They pay for biopsychosocial integrated care intervention. A potential advantage of the biopsychosocial integrated care intervention is that the costs per session will be lower than the costs per session of the multidisciplinary intervention in which multiple care providers are involved. Implementation is therefore expected to reduce the direct medical costs. Furthermore, the protocolled integrated care intervention consists of a restricted number of sessions, which prevents from extensive (likely unnecessary) treatments and the quicker start of treatment might prevent further chronification and even more difficult to treat disabilities. Whether or not the integrated care intervention will be eventually cost-effective as compared to the usual multidisciplinary care needs to be investigated still.

\section{INNOVATIVE PRODUCTS}

An integrated care approach is a novel approach that receives increasingly attention in the Netherlands. Until recently, health care was more or less subdivided into primary, secondary or tertiary care. Patients were referred to that specific health care which would fit best, based on the needs of the patient. In the last years, however, a new approach started to become more often used; the integrated care approach. The reason for developing such approach was that the number of patients treated in secondary and tertiary care settings increased quickly in the last years, causing the cost of specialized treatments provided in secondary and tertiary care settings to rise exponentially. It is expected that the increase in number of patients and associated costs will continue even further due to the aging population and the more demanding society. As described in chapter 6, an integrated care approach was developed as strategy to control the increasing costs and to offer patients an intervention with a similar biopsychosocial approach as would usually be offered within a multidisciplinary (secondary health care) programme. The idea of the Back on Track intervention was to provide the biopsychosocial intervention by trained physiotherapists within a primary physiotherapy practice, but under close direction and supervision of the referring physician in rehabilitation medicine; i.e. an integrated care approach.

\section{Network}

To be able to implement an integrated care approach, first a team of health care professionals should be recruited who are interested in being involved in an integrated care approach for patients with CLBP. Ideally, a small network within one area should be created as a starting point. This for example may include few GP's, physiotherapists, a department of rehabilitation medicine of a hospital and a rehabilitation centre. Each setting should appoint one leader who acts as primary contact, who stimulates the integrated care approach within their setting and takes overall responsibility. 


\section{Protocol}

One of the most important products necessary for implementation is the biopsychosocial integrated care intervention itself. Chapter 3 presented a detailed description of the Back on Track intervention. As presented in chapter 6 (feasibility study), the Back on Track intervention may use slight adaptations based on the recommendations provided. For example, an additional individual session (pain education) might be added and the physiotherapist may indicate whether a protocolled exposure session is required or not.

\section{Education programmes}

In order to be sure that physiotherapists deliver the protocol in a qualitatively sufficient way, it is important that physiotherapists have a biopsychosocial attitude (instead of a biomedical one) and are well informed about the procedure of an integrated care approach. One way to facilitate physiotherapists to work according to the protocol is by providing an education programme. Based on the findings of chapter 2 (systematic review) and chapter 6 (feasibility study), an education programme containing a few meetings only (2 or 3 sessions; 12 hours in total) can suffice, but only if physiotherapists receive additional support. Support can consist of a treatment protocol, video examples of complex situations, a website with information and frequently asked questions, and supervision over time to discuss difficulties (e.g. follow-up booster sessions). Within the education programme itself, physiotherapists need to be guided into how biopsychosocial elements can be practically provided and how to respond to certain situations. Discussing core-beliefs, cognitions, emotions, behaviour may be difficult. Therefore, the education programme should anticipate on this by providing practical training (e.g. role playing). The education program, which has previously been used for the Back on Track intervention, can be used in future with some small adaptations as just described. The education program should be offered as a program accredited by the Royal Dutch Society for Physical Therapy (KNGF) and offered by two experts minimally. Ideally, these experts have been involved in the development of the integrated care intervention and have clinical experience in providing interventions with a biopsychosocial approach.

Health care professionals involved in the integrated care network (e.g. GP's and physicians in rehabilitation medicine) also need to be educated about the biopsychosocial model of pain, the content of the biopsychosocial intervention as well as the role they have in the integrated care approach. It is of importance for the physician to know which patient to refer (what biopsychosocial profile), how patients need to be prepared for the intervention (what information to provide), when to contact the patient and physiotherapist during the intervention, and how to communicate (i.e. by phone, email, or digital communication system). One educational meeting for physicians prior to the start may suffice. This educational meeting needs to be developed and should be provided by the education team that is involved in the education programme of physiotherapists. After the physicians have received the education, it is important that the leader of each setting/department gains (and keeps) insight in the competence of each physician and contacts the educational team if additional training is necessary. 
While above described education programmes are relevant for working professionals, these are also relevant for upcoming health care professionals such as Physiotherapy students. Education about the biopsychosocial model should be well integrated in the curriculum to facilitate the development of a biopsychosocial orientation and attitude of students directly at the beginning of their study and career. To understand to what extent the biopsychosocial model is already merged into existing curricula, Universities of Applied Sciences should be contacted as a starting point. An overview should be created about what theory, practicums or other types of lessons are provided, and to what extent students learn from it and develop a biopsychosocial orientation and attitude. It is expected that such an overview will update our understanding about what role education about the biopsychosocial model has in existing curricula, and what is needed to maintain or improve it in future.

\section{Digital communication system}

As previously mentioned, an integrated care intervention requires collaboration of health care providers working in different institutes. In order to stimulate collaboration, good communication and transparency is required. Communication can be optimized with a digital communication tool. This digital communication tool should have a clear and easy to handle reporting format, enabling professionals to provide a quick overview of patient information and information about the progress of the therapy. In addition, this digital communication tool should include validated measurement instruments to evaluate the progress and quality of care. As soon as all health care professionals involved in the intervention have access to this communication tool, it will optimize transparency, and quality of care, and will furthermore lower the burden for patients. Due to the fact multiple digital communication tools already exist and different health care providers use different communication tools, it should first be identified which tools are already in use. It should be decided whether or not existing tools can be linked or a new tool needs to be developed.

\section{PLANNING \& REALISATION}

Before actually implementing a biopsychosocial integrated care intervention, a new study should be performed to compare the cost-effectiveness with a usual (multidisciplinary) secondary care intervention. One prerequisite for conducting a cost-effectiveness study is funding. At national level, organizations might be interested such as health care insurance companies, KNGF, or ZonMw which stimulates innovative research. In addition, other researchers and clinicians should be stimulated to apply for funding and to evaluate similar interventions. Increasing people's interest in this topic can be done (and is done) by transferring the available knowledge and findings at national and international conferences and symposia. The thesis findings are already presented at conferences such as the Pain Science in Motion Meetings (PSIM) in Brussels (Belgium) and Stockholm (Sweden); the 10th Congress of the European Pain Federation (EFIC) in Copenhagen (Denmark); International Back and Neck Pain Forum in Buxton (United Kingdom) and Oslo (Norway), and the symposium "Bruggen bouwen: vernieuwingen in de pijnrevalidatie" in Heerlen 
(the Netherlands). Apart from applying for funding and conducting a longitudinal study which may take several years, implementing an integrated care intervention will take a few more years. Within the first year, the network should be created by contacting and informing health care professionals. Furthermore, the Back on Track intervention should be revised, the education programme and the digital communication tool should be developed, and agreements should be made about the financial organization with the health care insurance companies. Within the subsequent years, professionals should be educated after which the Back on Track integrated care intervention can be implemented in daily practice. 


\section{Summary}





\section{SUMMARY}

This thesis mainly focuses on primary care physiotherapy treatments in patients with non-specific chronic low back pain (CLBP). Chapter 1 starts with a general introduction about CLBP, its definition, incidence and prevalence. It has been shown that a large proportion of people experience low back pain at some point in their lives and that one-quarter will develop chronic complaints (i.e. complaints with a duration of $\geq 3$ months). Although in most patients no specific pathology can explain the associated level of disability and pain, it has been suggested that biopsychosocial factors can act as an underlying mechanism. The underlying mechanism might relate to beliefs, attitudes and behaviours from individuals which can cause someone to become disabled at physical, psychological and social level. Recent therapy guidelines for low back pain recognise the possible impact of bio-psycho-social factors and recommend identifying and modifying these during therapy. The presence and influence of the psychosocial factors vary however between patients with CLBP. Depending on the influence of psychosocial factors, patients will be referred to and treated at a specific health care setting. Patients with more complex psychosocial factors might need (multi-, or interdisciplinary) secondary or tertiary care. Patients with less complex psychosocial factors might need primary care physiotherapy or advice only. Secondary care programs generally use evidence based cognitive behavioural approaches focusing on psychosocial factors, such as Graded Activity (GA), Exposure in vivo (EXP), and Acceptance and Commitment Therapy (ACT). Primary care programs on the other hand, generally include exercise and manual therapy. These therapies are often without or with a less sophisticated focus on psychosocial factors.

The effectiveness of a biopsychosocial approach using a cognitive-behavioural approach is well studied and shows moderate promising effects when provided multidisciplinary. Whether it will also have promising effects in primary care physiotherapy practices is unknown. No systematic review has yet been performed gathering the evidence for a biopsychosocial primary care intervention. Furthermore, most studies who did investigate the effectiveness of a biopsychosocial primary care intervention previously, did not select patients based on the complexity of the psychosocial complaints. New studies are required to investigate the effectiveness of a biopsychosocial primary care intervention in a homogeneous group of patients. To what extent a biopsychosocial primary care intervention would add benefit as compared to usual physiotherapy treatments in patients in which psychosocial complaints are of low complexity remains to be investigated (i.e. patients usually referred to physiotherapy treatments in primary care, classified as WPN2). In addition, it remains from an efficacy point of view (less costs, faster treatment) of interest to what extend a biopsychosocial primary care intervention would be beneficial for patients in which psychosocial complaints are of moderate complexity (i.e. patients usually treated by multidisciplinary teams in secondary care, classified as WPN3-). For this subgroup of patients, primary care may have some advantages over secondary care, such as: (1) accessibility is easier; (2) waiting time is mostly shorter; and (3) costs per session are lower.

In addition to patients treated conservatively, limited evidence is available about the effectiveness of a biopsychosocial approach in patients who receive invasive treatments such as lumbar spinal fusion surgery. Prehabilitation and post-operative rehabilitation seem to facilitate 
quicker regain in functioning. No guidelines are however available for e.g. the content and intensity of pre- and postoperative rehabilitation. Moreover, less is clear about what opinions spinal surgeons actually have about pre- and postoperative rehabilitation in patients undergoing lumbar spinal fusion surgery.

In Chapter $\mathbf{2}$ the evidence for a biopsychosocial intervention delivered by primary care physiotherapists to patients with CLBP was systematically reviewed. Furthermore, an overview was provided about the biopsychosocial intervention designs, profiles of physiotherapists who deliver the interventions and the training programs for the therapists. Three studies comparing a biopsychosocial intervention with education and advice showed moderate quality evidence that a biopsychosocial intervention is more effective in improving functional disability and pain than education and advice (i.e. at short-, medium, and long term). Four studies comparing a biopsychosocial intervention with a physical active intervention showed low quality evidence that a biopsychosocial intervention is equally effective in improving functional disability and pain as compared to a physical active intervention. In general, a biopsychosocial intervention seems promising if the biopsychosocial intervention includes a patient-centred format in which physical exercises are provided but most attention is paid to patient-specific goals, patient's cognitions and coping behaviour. The delivery of such an intervention with a biopsychosocial approach seems however challenging if physiotherapists are not experienced in delivering biopsychosocial elements and receive a rather short training program only. To ensure sufficient delivery of biopsychosocial elements, physiotherapists seemed to need additional support not only prior to, but also while delivering the intervention (i.e. offering a detailed protocol, DVD with examples of sessions, booster meetings and supervision). As general remark, it should be stressed that our systematic review included seven Randomised Controlled Trials (RCTs) of which two were (underpowered) pilot studies. We noticed that most studies did not assess the quality of the provided intervention or that physiotherapists did not optimally deliver the intervention. Due to these methodological and practical limitations, we concluded that future studies would be necessary to improve our understanding on the effectiveness of a biopsychosocial intervention provided by physiotherapists in primary care.

In Chapter $\mathbf{3}$ an overview of the development and content of the Back on Track intervention is provided. The Back on Track intervention was specifically developed for two subgroups of patients with CLBP who experience low to moderate complex psychosocial factors and who are low to moderately disabled in functioning (i.e. patients classified as WPN2 and WPN3-). We expected that these subgroups would benefit from a biopsychosocial primary care intervention when delivered by a physiotherapist trained in delivering cognitive behavioural principles. A team of health care professionals working in either primary or secondary care settings (i.e. physicians in rehabilitation medicine, psychologists, and physiotherapists) and patients from patient organisation "de Wervelkolom" were involved in the development of the Back on Track intervention. The intervention was based on recent literature and existing biopsychosocial multidisciplinary interventions provided at the department of Rehabilitation Medicine, Maastricht University Medical Centre (MUMC+) and Adelante Rehabilitation, Hoensbroek. The pain-consequence model was used as a format to identify biomedical factors (e.g. cause, diagnose, previous therapies) and 
behavioural factors (beliefs, emotions, attitudes, behaviour) which can have short and long term consequences at physical, cognitive, and social domain. The Back on Track intervention aims to identify these influencing bio-psycho-social factors in the first three individual sessions and stimulates patients to set patient-specific goals. In subsequent group session (eight in total), elements of cognitive-behavioural approaches are used such as Graded Activity (GA) and Exposure in vivo (EXP) to further identify patient's beliefs, emotions, attitudes and behaviour, and to improve a patient's level of functional activities. Group sessions, are further subdivided in educative group sessions, provided as rather theoretical/discussion sessions, and physical active group sessions, provided as active part; i.e. a stimulus to improve the level of activity and to become self-confident in being active. The Back on Track intervention ends with a final individual session with the physiotherapist in which the intervention and the progress of the patient is discussed. Afterwards, patients classified as WPN3- are structurally referred back to their physician in rehabilitation medicine for a final (reinforcing) consultation (i.e. resulting in an integrated care intervention). Patients classified as WPN2 are referred back if needed only.

After having specified the Back on Track intervention protocol, we defined the research protocol for our RCT which is described in Chapter 4. The RCT was set up as a double-blind, multicentred ( $n=8$ ) trial to compare the effectiveness and costs of the Back on Track intervention with usual primary care physiotherapy for patients with low complex psychosocial factors (i.e. classified as WPN2). A total sample size of $n=86$ was needed to detect a difference of $15 \%$ between groups on the Quebec Back Pain Disability Scale (functional disability). Physicians specialised in chronic pain treatments determined the biopsychosocial profile of a patient during a usual consultation and referred patients classified as WPN2 to the RCT. Patients received an intake with the research team to provide written consent and to complete baseline questionnaires. Afterwards, patients were randomised over two interventions; the Back on Track intervention or primary care physiotherapy as usual. Allocation was concealed for all except the research assistant. Furthermore, treatment was blinded for patients and data analysts. Functional disability was the primary outcome as measured with the Quebec Back Pain Disability Scale at post-treatment, 3 and 12 months of follow-up. Secondary outcomes included anxiety and depression, catastrophizing, pain intensity, kinesiophobia, self-efficacy, patient's global perceived effect, cost-effectiveness, and cost-utility estimated with cost diaries and quality-adjusted life years. In addition, credibility and expectancy were measured as potential prognostic factors. Planned analysis included linear mixed models, and incremental cost-effectiveness ratios and cost-utility ratios (plotted on a cost-effectiveness plane).

In Chapter $\mathbf{5}$ the results of the RCT are presented. Results showed that no differences in effects were found in improving functional disability between the Back on Track intervention and usual primary care physiotherapy at post-treatment and 3 months follow-up (NB: in patients classified as WPN2). No differences in effectiveness was furthermore shown in secondary outcomes such as pain, catastrophizing, anxiety, depression, self-efficacy and kinesiophobia. However, it should definitely be taken into account that the included sample size ( $n=25$ ) was insufficient to compare the interventions adequately and to draw firm conclusions. The conclusion that no differences in effectiveness are found between the interventions should therefore be interpreted with caution. It may be desirable to perform a new study with sufficient power. It is recommend- 
ed for future studies to reconsider the study design (e.g. a single subject design), recruitment strategy (e.g. via general practitioner or physiotherapy), and intervention design (e.g. identifying and better matching patient's expectations).

In Chapter $\mathbf{6}$ the results of our pilot study that was performed in addition to the RCT, are described. The pilot study aimed to evaluate the feasibility as well as the effectiveness of the integrated Back on Track intervention in patients with moderate complex psychosocial factors (i.e. patients classified as WPN3-). As mentioned previously, since physicians were structurally involved at the end of the intervention, so the program would result in an integrated care intervention. Physiotherapists who provided the intervention in the RCT also delivered the Back on Track intervention during the pilot study. The pilot study showed that trained primary care physiotherapists were able to sufficiently provide essential treatment elements of the Back on Track intervention. Furthermore, patients significantly improved their level of functional disability at post-treatment and 3 months follow up. Individual sessions including pain education were appreciated most by patients and physiotherapists. More time was recommended in addition to ensure sufficient delivery and understanding of the information. Unfortunately, as in the RCT, we faced difficulties with the recruitment of patients. The low recruitment rate resulted in extra waiting time for groups to start which negatively influenced the continuity of the intervention and motivation of patients. It is therefore recommended to offer group therapy only if there is sufficient supply of patients of interest. Furthermore, communication between physiotherapists and physicians was restricted and should be facilitated to strengthen the integrated care approach in future. Also, the value of a final consultation should be investigated since a low attendance rate with the physician was found in this study. Overall, a biopsychosocial primary care intervention seemed beneficial for patients with moderate complex psychosocial factors. A new study (e.g. with a single subject design or RCT) is recommended comparing the effects and costs of a biopsychosocial primary care intervention with a secondary care intervention as usual.

In Chapter $\mathbf{7}$ the opinions of Dutch and Swedish spinal surgeons about (biopsychosocial) pre- and postoperative rehabilitation in patients with CLBP undergoing lumbar spinal fusion surgery are described. This cross-sectional study showed that the opinions of spinal surgeons varied within two countries as well as between two countries. Variability in opinions was especially detected regarding the post-operative phase. The opinions and recommendations for functional activities, sport or work-related activities as well as for (biopsychosocial) therapy considerably varied (e.g. when to start, what is allowed or not allowed, what approach, or to what intensity). It was noticed that the need for research investigating the effectiveness of therapy strategies is high. This may increase uniformity between surgeons in future.

In Chapter 8 a general discussion is provided regarding the main findings of the studies. It was concluded that patients with CLBP benefit significantly more from a biopsychosocial primary care intervention than education and advice only. When a biopsychosocial primary care intervention is compared to a physically active (physiotherapy) program, inconclusive evidence was found. Apart from this, a biopsychosocial intervention seems feasible to be provided by primary care physiotherapists when training, supportive material and supervision is provided. Furthermore, patients who are usually referred to multidisciplinary interventions in secondary care (i.e. with moderate complex psychosocial complaints) seem to improve the level of disability sta- 
tistically significant after having received a biopsychosocial intervention offered as integrated care intervention by primary care physiotherapist and physicians in rehabilitation medicine. With regard to lumbar spinal fusion rehabilitation, a biopsychosocial approach seems less integrated in the pre- and postoperative rehabilitation according to current opinions of spinal surgeons' in the Netherlands and Sweden. Furthermore, variability in practice was reported, especially in the postoperative phase.

Based on above mentioned findings, future longitudinal studies are recommended to compare the (cost-) effectiveness of different therapy approaches in patients with CLBP. This will give final answer to whether a biopsychosocial primary care approach should be applied to patients with less complex psychosocial complaints, whether a biopsychosocial integrated care intervention or a multidisciplinary (biopsychosocial) secondary care intervention should be provided to patients with moderate complex psychosocial complaints, and what pre- and postoperative rehabilitation strategy (content, intensity, frequency and time point) is most beneficial to patients undergoing lumbar spinal fusion surgery. 

Samenvatting 



\section{SAMENVATTING}

Dit proefschrift richt zich voornamelijk op eerstelijns fysiotherapiebehandelingen voor patiënten met aspecifieke chronische lage rugpijn. Hoofdstuk 1 start met een algemene introductie over chronische lage rugpijn, de definitie, incidentie en prevalentie. Aangetoond is dat veel personen eens in hun leven lage rugpijn ervaren en dat een kwart hiervan chronische klachten ontwikkelt (dat wil zeggen klachten die 3 of meer maanden aanhouden). De mate van ervaren beperkingen en pijn kunnen in veel patiënten niet of niet volledig verklaard worden door de aanwezigheid van specifieke pathologie. Het blijkt dat biopsychosociale factoren als onderliggend mechanisme voor de mate van pijn en de ervaren beperkingen kunnen fungeren. Dit houdt in dat cognities, attitudes en gedrag van personen ertoe kunnen leiden dat iemand beperkt raakt op fysiek, psychologisch of sociaal vlak. Recente richtlijnen voor de behandeling van lage rugpijn erkennen de invloed van zowel biologische, psychologische als sociale factoren en adviseren deze factoren te identificeren en indien aanwezig te modificeren tijdens therapie. De aanwezigheid en invloed van psychosociale factoren kunnen echter van patiënt tot patiënt verschillen. Op basis van de mate van invloed van psychosociale factoren op het functioneren worden patiënten in de huidige zorg daarom verwezen naar een behandeling aangeboden in een instelling passend bij de ernst van de psychosociale problematiek. Patiënten waarbij de invloed van psychosociale factoren groter is, worden verwezen naar een (multi-, of interdisciplinair) tweede- of derdelijns behandeltraject. Patiënten waarbij de invloed van psychosociale minder groot is, worden verwezen naar een eerstelijns fysiotherapiebehandeling of ontvangen advies van hun behandelend arts. Behandelprogramma's in de tweede lijn bevatten over het algemeen een evidence-based cognitief-gedragsmatige aanpak welke gericht is op beïnvloeding van psychosociale factoren. Voorbeelden van dergelijke benaderingen zijn Graded Activity (GA), Exposure in vivo (EXP) en Acceptance and Commitment Therapy (ACT). Eerstelijns fysiotherapie behandelprogramma's aan de andere kant, bevatten vaak oefentherapie en/of manuele therapie. Deze behandelingen zijn over het algemeen minder gericht op het identificeren en modificeren van psychosociale factoren.

Behandelprogramma's met een cognitief-gedragsmatige aanpak gericht op het beïnvloeden van psychosociale factoren bleek vrij belovend wanneer multidisciplinair aangeboden. Of een biopsychosociale interventie ook effectief is wanneer deze monodisciplinair wordt uitgevoerd door eerstelijns fysiotherapeuten, is onbekend. Er is nog geen systematische review uitgevoerd die het bewijs voor een biopsychosociale eerstelijns interventie heeft verzameld en systematisch samengevat. Bovendien werden in de meeste onderzoeken naar de effectiviteit van een biopsychosociale eerstelijns interventie geen patiënten geselecteerd op basis van de complexiteit van de psychosociale factoren. Daarom zijn nieuwe studies nodig om de effectiviteit van een biopsychosociale eerstelijns interventie te onderzoeken in een homogene groep patiënten met chronische lage rugpijn. In hoeverre een biopsychosociale aanpak effectiever is dan regulier aangeboden eerstelijns fysiotherapie bij patiënten met chronische lage rugpijn en psychosociale factoren van lage complexiteit, moet nog onderzocht worden; dit zijn patiënten die normaliter worden verwezen naar een eerstelijns fysiotherapiebehandeling, en worden binnen de revalidatiegeneeskunde geclassificeerd als WPN2. Daarnaast is het vanuit doelmatigheidsoogpunt 
(minder kosten, snellere zorg) interessant om te onderzoeken of een dergelijk biopsychosociaal programma aangeboden door eerstelijns fysiotherapeuten voordeliger is bij patiënten met psychosociale factoren van matige complexiteit; deze patiënten worden normaliter behandeld in tweede- of derdelijns zorginstellingen, en worden binnen de revalidatiegeneeskunde geclassificeerd als WPN3-. Voor deze laatste subgroep van patiënten kan een eerstelijns fysiotherapeutisch behandelprogramma enkele voordelen hebben ten opzichte van een tweedelijns behandelprogramma, zoals: (1) makkelijkere toegankelijkheid, (2) over het algemeen kortere wachttijd en (3) lagere kosten per sessie.

Naast patiënten die conservatief worden behandeld, is er weinig bewijs voor het effect van een biopsychosociale aanpak bij patiënten die een invasieve behandelingen ondergaan, zoals een lumbale spinale fusieoperatie. Preoperatieve revalidatie (prehabilitatie) en postoperatieve revalidatie lijken het herstel in functioneren te faciliteren. Er zijn echter geen richtlijnen beschikbaar voor bijvoorbeeld de inhoud en intensiteit van pre- en postoperatieve revalidatie. Bovendien is weinig bekend over welke adviezen wervelkolomchirurgen eigenlijk geven met betrekking tot pre- en postoperatieve revalidatie.

In hoofdstuk $\mathbf{2}$ is het bewijs voor een biopsychosociale interventie aangeboden door eerstelijns fysiotherapeuten aan patiënten met chronische lage rugpijn, systematisch verzameld en weergegeven. Daarnaast is een overzicht gegeven van de designs van biopsychosociale interventies, de profielen van fysiotherapeuten die de interventies gaven, en de scholingsprogramma's die de therapeuten ontvingen. Drie studies waarin een biopsychosociale interventie werd vergeleken met educatie en advies, toonden matig kwalitatief bewijs dat een biopsychosociale interventie effectiever is in het verbeteren van functionele beperkingen en pijn dan alleen educatie en advies (dat wil zeggen op korte, middellange en lange termijn). Vier studies waarbij een biopsychosociale interventie met een fysieke actieve interventie werd vergeleken, toonden laag kwalitatief bewijs dat een biopsychosociale interventie even effectief is in het verbeteren van functionele beperkingen en pijn in vergelijking met een fysiek actieve interventie. Over het algemeen lijkt een biopsychosociale interventie veelbelovend indien de biopsychosociale interventie een patiëntgericht format bevat waarin fysieke oefeningen worden gegeven, maar de meeste aandacht wordt besteed aan patiënt-specifieke doelen, cognities en gedrag. Het geven van een dergelijke interventie met een biopsychosociale aanpak lijkt echter uitdagend wanneer fysiotherapeuten geen ervaring hadden met het geven van psychosociale elementen en slechts een vrij kort trainingsprogramma ontvingen. Om te zorgen dat fysiotherapeuten competent zijn in het toepassen van de biopsychosociale elementen, lijken fysiotherapeuten niet alleen voorafgaand aan, maar ook tijdens het geven van de interventie extra ondersteuning nodig te hebben; bijvoorbeeld door middel van een gedetailleerd protocol, dvd met voorbeelden van sessies, boosterbijeenkomsten en begeleiding. Algemeen dient te worden benadrukt dat de systematische review zeven gerandomiseerde gecontroleerde trials (RCTs) bevatte, waarvan twee ('underpowered') pilotstudies. Opvallend is dat de meeste studies niet hebben beoordeeld wat de kwaliteit van de gegeven interventie was of rapporteerden dat fysiotherapeuten de interventie niet optimaal hebben geleverd. Door deze methodologische en praktische beperkingen is geconcludeerd dat toekomstige studies nodig zijn om meer inzicht te krijgen in de effectiviteit van een biopsychosociale interventie aangeboden door eerstelijns fysiotherapeuten. 
In hoofdstuk 3 wordt een beschrijving gegeven van de ontwikkeling en inhoud van de Back on Track interventie. De Back on Track interventie is specifiek ontwikkeld voor twee subgroepen van patiënten met chronische lage rugpijn die psychosociale factoren van lage tot matige complexiteit ervaren en weinig tot matig beperkt zijn in het uitvoeren van dagelijkse functionele activiteiten (dat wil zeggen, patiënten geclassificeerd als WPN2 en WPN3-). Verwacht werd dat deze subgroepen profiteren van een biopsychosociale interventie in de eerste lijn geleverd door een fysiotherapeut getraind in het aanbieden van cognitief-gedragsgeoriënteerde principes. Een team van zorgverleners werkzaam in een eerste- of tweedelijns zorginstelling (revalidatieartsen, psychologen en fysiotherapeuten) en patiënten van de patiëntenorganisatie "de Wervelkolom" waren betrokken bij de ontwikkeling van de Back on Track interventie. De interventie is gebaseerd op recente literatuur en bestaande biopsychosociale multidisciplinaire interventies die worden aangeboden op de afdeling Revalidatiegeneeskunde, Maastricht Universitair Medisch Centrum + $(\mathrm{MUMC}+)$ en Adelante Revalidatie, Hoensbroek. Het pijn-gevolgenmodel is gebruikt als format om biomedische factoren te identificeren (de oorzaak, diagnose, eerdere therapieën) en gedragsfactoren (overtuigingen, emoties, attitudes, gedrag) die korte en lange termijn gevolgen hebben op fysiek, cognitief en sociaal vlak. De Back on Track interventie heeft tot doel deze invloedrijke bio-psycho-sociale factoren in de eerste drie individuele sessies te achterhalen en stimuleert de patiënt om patiënt-specifieke doelen vast te stellen. In de daaropvolgende groepssessies (acht in totaal) worden elementen van cognitief-gedragsmatige principes gebruikt, zoals Graded Activity (GA) en Exposure in vivo (EXP). Deze principes worden toegepast om de cognities, emoties, attitudes en gedrag van patiënten verder te identificeren en modificeren, en het niveau het functionele activiteitenniveau te verhogen. Groepssessies worden verder onderverdeeld in educatieve groepssessies (aangeboden als theoretische sessies), en fysieke actieve groepssessies (aangeboden als actief onderdeel; dat wil zeggen, een stimulans om het niveau van activiteiten te verbeteren en zelfverzekerd te worden in het actief zijn). De Back on Track interventie eindigt met een laatste individuele sessie gegeven door de fysiotherapeut waarin de interventie en de voortgang van de patiënt worden besproken. Daarna wordt een WPN3- patiënt structureel terugverwezen naar zijn/haar verwijzend revalidatiearts voor een laatste (bekrachtigend) consult. Hierdoor ontstaat een anderhalvelijns zorgtraject. Patiënten geclassificeerd als WPN2 worden enkel terugverwezen indien nodig.

$\mathrm{Na}$ de ontwikkeling van het behandelprotocol voor de Back on Track interventie, is het onderzoeksprotocol voor de RCT opgesteld, beschreven in hoofdstuk 4. De RCT is opgezet als een dubbel geblindeerd, multicenter $(n=8)$ studie om de effectiviteit en de kosten van de Back on Track interventie te vergelijken met reguliere eerstelijns fysiotherapie voor patiënten met laag complexe psychosociale factoren (geclassificeerd als WPN2). Een totale steekproefgrootte van n $=86$ was berekend om een verschil van 15\% te detecteren tussen groepen op de Quebec Back Pain Disability Scale (functionele beperkingenniveau). Revalidatieartsen gespecialiseerd in chronische pijnrevalidatiebehandelingen, werden gevraagd tijdens een regulier consult het biopsychosociale profiel van een patiënt te bepalen en patiënten geclassificeerd als WPN2 te verwijzen naar de RCT. Patiënten werden uitgenodigd voor een intake met het onderzoeksteam om schriftelijke toestemming te geven en baseline vragenlijsten in te vullen. Daarna werden de patiënten gerandomiseerd over twee interventies; de Back on Track interventie of eerstelijns fysiotherapie 
zoals regulier wordt aangeboden. De randomisatielijst was voor iedereen verborgen gehouden ('concealed allocation'), behalve voor de onderzoeksassistent. Bovendien werden de patiënten en data analisten gedurende het onderzoek geblindeerd voor de aan iedere patiënt toegewezen behandeling. Het functionele beperkingenniveau was de primaire uitkomstmaat gemeten met de Quebec Back Pain Disability Scale direct na het afronden van de behandeling en na 3 en 12 maanden na het einde van de behandeling. Secundaire uitkomstmaten waren angst en depressie, catastroferen, pijnintensiteit, kinesiofobie, vertrouwen in eigen kunnen, waargenomen effect van de patiënt, kosteneffectiviteit en kostenutiliteit berekend met kostendagboeken en kwaliteit gecorrigeerde levensjaren (quality-adjusted life-years). Daarnaast werden de geloofwaardigheid en verwachtingen gemeten als potentiële prognostische factoren. De geplande analyse was een lineaire mixed-model analyse, en incrementele kosteneffectiviteitsratio en kostenutiliteitsratio (uitgezet op een kosteneffectiviteitsvlak).

In hoofdstuk 5 worden de resultaten van de RCT gepresenteerd. Op basis van de resultaten werd geen verschil in effectiviteit aangetoond in het verbeteren van functionele beperkingen tussen de Back on Track interventie en de regulier aangeboden eerstelijns fysiotherapie behandeling direct na behandeling en na 3 maanden follow-up (NB: bij patiënten geclassificeerd als WPN2). Bovendien werd geen verschil in effectiviteit gevonden in secundaire uitkomstmaten zoals pijn, catastroferen, angst, depressie, vertrouwen in eigen kunnen en kinesiofobie. Er moet echter wel rekening gehouden worden met het feit dat het aantal geïncludeerde patiënten $(\mathrm{n}=$ 25) ontoereikend was om de interventies adequaat te vergelijken en om definitieve conclusies te kunnen trekken. De conclusie dat er geen verschil in effectiviteit is gevonden tussen de interventies, dient daarom met voorzichtigheid te worden geïnterpreteerd. Het is wenselijk om een nieuwe studie met voldoende power uit te voeren. Voor toekomstige studies is aanbevolen om zowel het studiedesign (bijvoorbeeld een single subject design), de wervingsstrategie (bijvoorbeeld via huisarts of fysiotherapeut) en het design van de interventie (bijvoorbeeld het identificeren van en matchen met de verwachtingen van de patiënt) te heroverwegen.

In hoofdstuk 6 worden de resultaten van de pilotstudie beschreven. Deze studie is naast de RCT uitgevoerd. De pilotstudie was gericht op het evalueren van de haalbaarheid en de effectiviteit van de anderhalvelijns Back on Track interventie bij patiënten met matig complexe psychosociale factoren (patiënten geclassificeerd als WPN3-). Aangezien, zoals eerder vermeld, revalidatieartsen structureel betrokken waren aan het eind van de interventie, kan dit programma geclassificeerd worden als een anderhalvelijns zorgtraject. Fysiotherapeuten die de interventie in de RCT leverden, leverden ook de Back on Track interventie tijdens de pilotstudie. De pilotstudie toonde aan dat getrainde fysiotherapeuten in de eerste lijn voldoende in staat waren om essentiële behandelelementen van de Back on Track interventie te leveren. Bovendien verbeterde het niveau van functionele beperkingen van de deelnemende patiënten statistisch significant direct na de behandeling en na 3 maanden follow-up. De individuele sessies waaronder pijneducatie werden het meest gewaardeerd door zowel patiënten als fysiotherapeuten. Meer tijd voor pijneducatie werd echter aanbevolen om er voor te zorgen dat de informatie voldoende begrepen wordt. Helaas ondervonden we, net als in het RCT, problemen bij de werving van patiënten. De trage werving resulteerde in extra wachttijd voor groepen hetgeen vervolgens de continuïteit van de interventie en de motivatie van patiënten negatief beïnvloedde. Aanbevolen is daarom 
om alleen groepstherapie aan te bieden als er voldoende aanmeldingen van geschikte patiënten zijn. Bovendien was de communicatie tussen fysiotherapeuten en revalidatieartsen beperkt en zal deze in de toekomst gefaciliteerd moeten worden om de anderhalvelijns zorgketen te versterken. Ook moet de toegevoegde waarde van een afsluitend consult met de revalidatiearts onderzocht worden, aangezien de aanwezigheid van patiënten tijdens dit consult laag was. In het algemeen leek een biopsychosociale eerstelijns interventie gunstig voor patiënten met matige complexe psychosociale problematiek. Een nieuwe studie (bijvoorbeeld met een single subject design of $R(T)$ is aanbevolen om de effecten en kosten van een biopsychosociale eerstelijns interventie te vergelijken met een tweedelijns interventie zoals op dit moment in de reguliere zorg wordt aangeboden.

In hoofdstuk 7 worden de meningen van Nederlandse en Zweedse wervelkolomchirurgen over (biopsychosociale) pre- en postoperatieve revalidatie bij patiënten met chronische lage rugpijn die een lumbale spinale fusieoperatie ondergaan beschreven. Deze cross-sectionele studie toonde aan dat de meningen van wervelkolomchirurgen varieerden, zowel in beide landen alsook tussen de landen. Verschil van meningen waren met name zichtbaar in de postoperatieve fase. De adviezen en aanbevelingen voor functionele activiteiten, sport- of werk gerelateerde activiteiten en (biopsychosociale) therapieën varieerden aanzienlijk. Bijvoorbeeld, wanneer gestart mag worden na de operatie, wat is toegestaan of niet toegestaan, welke therapie-aanpak (biopsychosociaal of niet) en met welke intensiteit. Duidelijk is geworden dat onderzoek naar de effectiviteit van verschillende strategieën noodzakelijk is. Dit vergroot ook de mogelijkheid om in de toekomst te komen tot evidence based-uniformiteit tussen wervelkolomchirurgen.

In hoofdstuk 8 is een algemene discussie weergegeven over de belangrijkste bevindingen van de studies. Geconcludeerd is dat patiënten met chronische lage rugpijn significant meer profiteren van een biopsychosociale eerstelijns interventie dan educatie en advies. Wanneer een biopsychosociale eerstelijns interventie wordt vergeleken met een fysiek actief (fysiotherapeutisch) programma, is onvoldoende bewijs gevonden voor een verschil in effectiviteit. Voor eerstelijns fysiotherapeuten blijkt het wel haalbaar een biopsychosociale interventie te geven wanneer zij training, ondersteunend materiaal en begeleiding ontvangen. Bovendien verbeteren patiënten die normaliter worden verwezen naar een multidisciplinaire tweedelijns interventie (patiënten met matig complexe psychosociale klachten) het niveau van functionele beperkingen statistisch significant nadat zij een biopsychosociale interventie ontvangen die wordt aangeboden als een anderhalvelijns interventie door revalidatieartsen en eerstelijns fysiotherapeuten. Wat betreft de revalidatie van de lumbale spinale fusieoperatie, lijkt een biopsychosociale aanpak minder geïntegreerd in de pre- en postoperatieve revalidatie volgens de huidige adviezen van wervelkolomchirurgen in Nederland en Zweden. Bovendien lijkt er veel variëteit te zijn in aanpak, vooral in de postoperatieve fase.

Uitgaande van bovengenoemde bevindingen wordt aanbevolen in de toekomst longitudinale studies uit te voeren om de (kosten) effectiviteit van verschillende therapieën bij patiënten met chronische lage rugpijn te vergelijken. Dit zal een definitief antwoord geven op vragen zoals: moet een biopsychosociale eerstelijns interventie worden toegepast bij patiënten met laag complexe psychosociale klachten; moet een biopsychosociale anderhalvelijns interventie dan wel een multidisciplinaire (biopsychosociale) tweedelijns interventie worden toegepast bij pati- 
enten met matig complexe psychosociale klachten; en, welke pre- en postoperatieve revalidatie strategie (inhoud, intensiteit, frequentie en startmoment) is het meest voordelig bij patiënten die lumbale spinale fusieoperatie ondergaan. 
Dankwoord 



\section{DANKWOORD}

Na vier jaar is het dan eindelijk zo ver; het promotietraject is afgerond. Wat een gekke gedachte is dat. Maar eigenlijk is het pas echt afgerond wanneer ik iedereen bedankt heb voor alle steun en toeverlaat de afgelopen jaren. Het klinkt behoorlijk cliché, maar het is echt zo, zonder jullie had ik dit nooit gekund.

Allereerst wil ik mijn promotieteam Rob, Jeanine en Ivan bedanken. Rob, ik was ontzettend blij met jou als eerste promotor. Jij stond voor het onderzoek en zocht op ieder (on)mogelijk moment een gaatje om je in te zetten als promotor, onderzoeker of als revalidatiearts. Doordeweeks, in het weekend of in de avonden, het maakte je allemaal niet uit. Ik kon op je rekenen en dat was fijn. Daarnaast lukte het je op de een of andere manier altijd om mij te motiveren op momenten dat mijn vertrouwen even tot het nulpunt gezakt was. Dat was ontzettend fijn! Jeanine, ik weet nog als de dag van vandaag dat jij, samen met Ivan, het sollicitatiegesprek voerde en mij aannam als onderzoeker op de afdeling. Bedankt voor deze geweldige uitdaging. Tijdens de afgelopen vier jaar was het fijn samenwerken met je. Wanneer nodig, stond jij voor me klaar. In de laatste periode heb ik je ook als vakgroep voorzitter mogen meemaken. Ik bewonder hoe je die taak op je genomen hebt! De allerbelangrijkste van het stel was Ivan. Ivan, wij zagen elkaar iedere week. En zo niet, dan belden we elkaar wel. Jij was ook eerder mijn dagelijkse begeleider dan mijn wekelijkse begeleider. lemand die na een minder leuk overleg nog altijd even vroeg hoe ik me voelde en hoe ik vond dat het was gegaan. Bedankt voor deze fijne begeleiding. En, bedankt voor de ontelbare tips die je me hebt gegeven, de snelle feedback, de uren frustraties als het weer eens tegen zat, maar vooral ook bedankt voor de gezellige momenten tijdens ons overleg die structureel te lang duurden, de grappen en steken onder water, de lunch wandelingen (oh nee), en de gezelligheid op de borrels en congressen.

Marion, jij was de onmisbare kracht achter de schermen. En dat terwij jij nog 5 andere onderzoeken draaiende hield (of nog meer?!). Ik snap nog steeds niet hoe je dat doet. De woensdag was onze dag waarop we elkaar zagen, een dag van uitersten. We hebben heel wat gebaald en gejuicht, en weer gebaald en gejuicht. Het was een hele uitdaging om het onderzoek draaiende te houden. Maar, ik was blij dat ik het samen met jou mocht doen. En nu, nu kunnen we samen juichen dat het einde daar is. Dankjewel voor alles!

Margareth, ook jij was een grote steunpilaar. lemand waar ik altijd bij kon binnen lopen voor een vraag of een goed gesprek. Jouw uitnodigende blauwe stoel op de kamer zal ik missen. Daarnaast stond jij erop dat iedereen fit en betrokken bleef. De dagelijkse wandelingen, sportactiviteiten, yoga middagen, kerstlunches, koffiemomentjes en de carnavalsuitjes waren niet te missen. Dankjewel hiervoor!

Albère; manusje van alles. Van het ontwikkelen van het behandelprogramma, het geven van scholingen, het schrijven van artikelen, tot het opdoen van nieuwe ideeën; jij was erbij. Ik heb ontzettend veel aan jou gehad en vooral ook veel van je geleerd. Ik zag jou als iemand die op een luchtige manier kritisch discussieert, brainstormt met humor en zich daarnaast inzet zonder gemaar. Voor mij was jij een groot voorbeeld. Dankjewel! 
Alle andere collega's van de vakgroep Mariëlle, Jeroen, Inge, Marlies, Henk, Eugène, Yvonne en Clemens, externe en interne PhD's en de collega's van Adelante Kenniscentrum; BEDANKT! Dankzij jullie heb ik een ontzettend gezellige en inspirerende tijd gehad! In het bijzonder wil ik mijn (oud-)kamergenootjes bedanken: Carolien, Vera B., Vera M., Laura, Mellanie, Cynthia, Thijs, Charlotte, Ryanne, Max, Sanne, Renée, Ramon, Julia en Veronique. Een aantal van jullie zag ik bijna dagelijks. Bedankt voor de gezellige Fika's en vooral de positieve sfeer in onze kamer. Met z'n allen knalden we erop. Niks was te gek. Mijn laatste "That was easy" is nu (bijna) aangebroken, maar ik hoop dat jullie hem nog vaak aantikken.

Carolien, een special thanks to you, want tegelijkertijd begonnen wij ons PhD-traject. Bijna vier jaar lang hebben we tegenover elkaar gezeten, dezelfde stappen doorlopen, de theepot gedeeld en vooral, alle challenges doorstaan. Naast de gezamenlijke buikspier-, arm- en bil-challenges, heb jij er zelf nog een aantal achteraan geplakt. Van het organiseren van je bruiloft, het kopen en verbouwen van je huis, tot aan het zwanger zijn en het opvoeden van Robin; je hebt het allemaal gedaan. En dat met die ultieme rust die je uitstraalt. Lieve Carolien, jij bent naast een fijne collega ook een fijne vriendin. Eentje met goede adviezen en tips, eentje met een luisterend oor. Ik ben daarom ontzettend blij dat jij straks als paranimf naast me staat.

Vera B., samen zaten we in hetzelfde schuitje, op weg naar de eindstreep. En jeetje, wat ging jij als een speer. Ik keek mijn ogen uit hoe goed en gemakkelijk het bij jou ging. Ik weet zeker dat je in de toekomst een super gemotiveerde gepromoveerde revalidatiearts zult zijn. You can do it! Vera M. jij was mijn vaste UM-avond/diner-maatje die ervoor zorgden dat de avonden op de UM naast gezellig ook nog eens productief waren. Het was fijn dat je er in de eerste twee jaren van mijn traject was! En, hoe leuk is het nu om elkaar nu weer te treffen op de HAN?! Inge, met jou was het lekker down to earth, lekker relaxt. Als je op de vakgroep was, stak je altijd even je hoofd onze kamer binnen om 'hoi' te zeggen. Ook heb je me wanneer het uitkwam even uit de sleur getrokken voor een kop koffie. Dankjewel! Marlies, naast het feit dat jij mij geholpen hebt met de scholing en het schrijven van het behandelartikel, heb jij me door de laatste loodjes van mijn promotie gesleept. We hebben twee schrijfweken in ons leven geroepen en ons afgezonderd van de wereld om ons proefschrift af te ronden. Met z'n tweeën voelde het toch minder zielig. Dankjewel voor de gezelligheid, jouw input, jouw doorzettingsvermogen die mij ook weer gestimuleerde om door te gaan (zelfs op koningsdag met live muziek op de achtergrond), het delen van de frustraties en vooral de grappige momenten (koffiezetten 2.0).

Revalidatieartsen van het MUMC+, en in het bijzonder Robin en Marieke, bedankt voor het screenen en doorsturen van de patiënten naar het onderzoek. Het was een hele puzzel voor jullie om alle onderzoeken uit elkaar te houden, maar het was fijn dat jullie klaarstonden! Christoph, baliemedewerksters en secretariaat van de afdeling; ik vond het een uitje om tijdens de inclusieperiode bij jullie langs te komen. Bijna iedere week keken we of er nog nieuwe aanmeldingen waren en of we er nog iets aan konden doen. Jullie stonden voor me klaar, ondanks alle drukte en hectiek. Bedankt voor jullie hulp! Ook afdeling Orthopedie en Anesthesiologie, bedankt voor de fijne samenwerking. Vooral Paul en Mieke, dankjewel voor het meedenken tijdens het onderzoek en de korte praatjes die ik regelmatig mocht houden op jullie afdeling. Dit heeft ons onderzoek een stap vooruitgeholpen. 
Uiteraard gaat ook mijn dank uit naar alle patiënten die hebben deelgenomen aan het onderzoek. Zonder hun was ik natuurlijk helemaal nergens. Bedankt voor de inzet, het volgen van het behandelprogramma en het invullen van vragenlijsten. Ook Huis voor de zorg, bedankt voor het meedenken voorafgaand aan het onderzoek, alsook patiëntenvereniging 'de Wervelkolom' voor de bijdrage aan de ontwikkeling van het behandelprogramma.

Verder wil ik alle andere coauteurs bedanken voor hun inzet; Marluuke Jakobs, Jos Kleijnen, Jetse Jelsma, Paul Willems, Mari Lundberg, Ton Ambergen en Frans Abbink. Samen met jullie hebben we verschillende artikelen tot een mooi geheel kunnen brengen. Dankjulliewel! Frans, jij bent tevens betrokken geweest bij het ontwikkelen van het behandelprogramma en het uitvoeren hiervan. Ik vind het knap hoe actief jij naast jouw praktijk voor het vak fysiotherapie staat. Ik wil jou, alsook alle andere fysiotherapeuten van Fysiohof/Fysio043, ICL fysio, Fysio Margraten, Fysiotherapie Giessen-Ploemen, Fysio Zuyd Caberg, Fysio Valkenburg, Fysiotherapiepraktijk Yvonne Janss ontzettend bedanken voor jullie inzet tijdens het onderzoek.

De beoordelingscommissie Prof. dr. Dirk Ruwaard, Prof. dr. Rob de Bie, dr. Mariëlle Goossens, Prof. dr. Raymond Ostelo en Prof. dr. Daniëlle van der Windt; bedankt voor de tijd en moeite die jullie hebben gestoken in het lezen en beoordelen van dit proefschrift.

Kalemina's Marjan, Roos, Kiki, Romee, Milou en Chloé jullie waren mijn tweede familie. Bij jullie kwam ik 6 jaar lang thuis. Samen eten, films kijken, kletsen en sporten. Het was ontzettend fijn wonen. Dankjulliewel voor deze gezellige jaren!

Jaarclub-vriendinnetjes van Diez D'oro; Inge, Kyra, Liselot, Ine, Michelle, Milea, Sanne en Iris, met jullie is het Maastrichtse avontuur begonnen. Mede dankzij jullie heb ik de tijd van mijn leven gehad in Maastricht. Van de gezellige feestjes tot aan de degelijke thee-avonden met Hollands Next Topmodel op de achtergrond. Het zorgde voor een goede balans tijdens het studeren en mijn PhD-traject. Het was een Top-tijd! Een bijzonder dankjewel voor diehards Lise (van NP) en Ine. Jullie waren erbij tot aan de laatste week. Dankjewel voor jullie steun, luisterend oor, het JC-huis (super fijn, Ine!) en de gezellige afsluiting!

GW-vriendinnetjes Ilse en Adriënne (wooeeh-aaahh); dankjulliewel voor de hilarische momenten tijdens de studie en daarna. Het was om te gieren! Rosanne en Dorien; onze etentjes waren vaak onmogelijk te plannen, en het menu vaak moeilijk samen te stellen, maar het was een gezellige boel als het lukte. Bedankt voor de ontspanning! Hannah, tijdens onze Master-stageperiode bij Maarten zijn we samen besmet geraakt met het onderzoeks-virus. Het was leuk dat we allebei in Maastricht bleven om als promovendus aan de slag te gaan. Menig koffietjes hebben we hierdoor kunnen nuttigen met als doel bij te kletsen over het werk, maar vooral ook over alle levensgebeurtenissen, nieuwtjes en avonturen. Bedankt!

Niet te vergeten wil ik ook alle vrienden en vriendinnen van thuis bedanken voor alle Veghelse gezelligheid tijdens de weekenden. Alle BB\&B's, BFF's, verjaardagsfeesten, carnavalsuitjes, festivals en vriendenweekenden hebben ertoe bijgedragen dat ik mijn gedachten kon verzetten en opgeladen (?) weer aan de werkweek in Maastricht kon beginnen. Mai, Marieke en Sjoerd, jullie hebben me doordeweeks ook nog vaak gesteund met de gebruikelijke koffiemomenten en filmavonden. Na even mijn verhaal kwijt te hebben gekund, kon ik na deze stop weer fanatiek verder. Dankjewel! 
Sjoerd, jou wil ik nog even in het bijzonder bedanken. Jij bent mijn beste maatje. Een maatje waarmee ik goede gesprekken kan hebben, maar vooral ook kan lachen. Jij hebt mij het Maastrichtse leven geïntroduceerd waar we regelmatig met veel plezier (zingend, dansend, feestend) tijd hebben doorgebracht. Ik wil je bedanken voor je steun de afgelopen jaren, het laatste loodjes pakket (deze heeft me erdoorheen gesleept), je hulp tijdens de voorbereidingen voor de grote dag en het feit dat je mijn paranimf wilt zijn. Hier ben ik super blij mee.

Lieve Pap en mam, jullie ben ik immens dankbaar. Jullie zijn er altijd. Jullie hebben me vanaf jongs af aan gestimuleerd te doen wat ik graag wil doen, door te gaan wanneer ik graag wil doorgaan. Ik mag van geluk spreken zo'n ontzettend lieve, gemotiveerde ouders te hebben. Wat ik hier bereikt heb, is allemaal dankzij jullie. Dankjewel lieve pap en mam!

Su en Nieke, jullie zijn mijn lieve zussen en allerbeste vriendinnen. Met jullie kan ik lachen en mopperen tegelijk. Dankjewel voor wie jullie zijn. Samen met Joran, Pierre de kleine deugniet Ise en newborn Jurre hebben jullie mij door de drukke periode heen gesleept. Dankjewel voor jullie steun en vooral jullie geduld het afgelopen jaar. Want, we hebben elkaar regelmatig moeten beloven dat ik het later nog wel zouden inhalen. Maar we weten, dat gaat zeker gebeuren.

Trudy, Chris, Roel, Sil, Sophie, Emma, Moniek en Twan, bij jullie zijn voelt als een warm bad. Zelfs toen Bart nog in Zweden zat. Jullie zijn altijd oprecht geïnteresseerd. En, aan gezelligheid geen gebrek. De menig speciaal biertjes en kaasplankjes hebben voor een goede balans gezorgd. Bedankt voor deze fijne afwisseling in de drukke tijd!

Last but not the least: Bart, mijn allergrootste en onmisbare steun. Zonder jou had ik dit nooit gekund. Of je nu in Zweden of in Nederland zat, in rustige of drukke tijden, jij was er voor me. Jij stond achter me en hebt me gestimuleerd door te zetten, te focussen, keuzes te maken en prioriteiten te stellen. Vooral dat laatste was een grote uitdaging voor me. Want, alles in het leven is leuk, toch? En ik weet, jij hebt hierdoor heel wat moeten doorstaan. Ik ben blij dat we vanaf nu eindelijk echt samen kunnen zijn. In Nederland, in Veghel, in ons droomhuisje. Ik hoop dat dit nooit meer veranderd. 
About the author 



\section{ABOUT THE AUTHOR}

Reni was born on August 19th 1988 in Veghel, the Netherlands. She attended secondary general education at Zwijsen College in Veghel after which she started a Bachelor study Physiotherapy at Fontys University of Applied Sciences in Eindhoven. In 2009 she obtained her Bachelor of Science degree and started to work as a physiotherapist. Next to her work as physiotherapist, she directly enrolled in a new study Health Sciences at Maastricht University. In 2013, she obtained her 2nd Bachelor of Science degree, now in the field of Human Movement Sciences. After that, she continued her studies with the Master Biology of Human Performance and Health at Maastricht University. Having obtained her Master of Science degree she started to work as fulltime researcher at the Department of Rehabilitation Medicine at Maastricht University and stopped working as a physiotherapist. During the first two years she worked as junior researcher. Her main responsibilities during that period were to prepare, develop and conduct the Back on Track project. After the first two years, Reni became appointed as a PhD candidate to continue the Back on Track project and the spinal fusion project. The results of both projects are presented in this thesis. Since September 2017, Reni works at the Master Physician Assistant at the HAN University of Applied Sciences. She is involved in the entire curriculum, but her main role is to supervise students in methodological and practical aspects of scientific research, and how research findings can be interpreted and used for clinical practice. From November 2017, Reni will be a member of the research group Organization of Healthcare and Services from the HAN University of Applied Sciences. 


\section{PUBLICATION LIST}

van Erp RMA, Huijnen IPJ, Verbunt JA, Smeets RJEM. A biopsychosocial primary care intervention (Back on Track) versus primary care as usual in a subgroup of people with chronic low back pain: protocol for a randomised, controlled trial. J Physiother. 2015;61(3):155. doi: 10.1016/j. jphys.2015.03.003

van Erp RMA, Huijnen IPJ, Smeets RJEM. Chapter 15. Graded Exposure in de eerste lijn. Book Graded Exposure. Houten: Bohn Stafleu en van Loghum. 2017.

van Erp RMA, Huijnen IPJ, Köke AJA, Abbink FE, den Hollander M, Smeets RJEM. Development and content of the biopsychosocial primary care intervention 'Back on Track' for a subgroup of people with chronic low back pain. Physiotherapy. 2017 Jun;103(2):160-166. doi: 10.1016/j.physio.2016.04.004.

van Erp RMA, Jelsma J, Huijnen IPJ, Lundberg M, Willems PC, Smeets RJEM. Spinal surgeons' opinions on pre- and postoperative rehabilitation in patients undergoing lumbar spinal fusion surgery: a survey-based study in the Netherlands and Sweden. Accepted, Spine 2017.

van Erp RMA, Huijnen IPJ, Jakobs MLG, Kleijnen J, Smeets RJEM. Effectiveness of primary care interventions using a biopsychosocial approach in chronic low back pain: a systematic review. Submitted.

van Erp RMA, Huijnen IPJ, Ambergen AW, Verbunt JA, Smeets RJEM. Biopsychosocial primary care and physiotherapy as usual show no differences in effects in patients with chronic low back pain: results of a randomized controlled trial. Submitted.

van Erp RMA, Huijnen IPJ, Köke AJA, Verbunt JA, Smeets RJEM. Feasibility of the biopsychosocial primary care intervention 'Back on Track' for patients with chronic low back pain: a process and effect-evaluation. Submitted. 